\title{
Bayesian Model Selection and Parameter Estimation For A Nonlinear Fluid-structure Interaction Problem
}

\author{
by
}

\author{
Rimple Sandhu \\ A thesis submitted to \\ the Faculty of Graduate and Postdoctoral Affairs \\ in partial fulfilment of \\ the requirements for the degree of
}

\section{Master of Applied Science}

in

Civil Engineering

\author{
Carleton University \\ Ottawa, Ontario, Canada \\ January 2013
}
The Master of Applied Science in Civil Engineering is a joint program with the University of Ottawa, administered by the Ottawa-Carleton Institute for Civil Engineering

Copyright (C)

2012 - Rimple Sandhu

All rights reserved 
Library and Archives

Canada

Published Heritage

Branch

395 Wellington Street

Ottawa ON K1A ON4

Canada
Bibliothèque et

Archives Canada

Direction du

Patrimoine de l'édition

395 , rue Wellington

Ottawa ON K1A ON4

Canada
Your file Votre référence

ISBN: 978-0-494-94653-4

Our file Notre référence

ISBN: 978-0-494-94653-4
NOTICE:

The author has granted a nonexclusive license allowing Library and Archives Canada to reproduce, publish, archive, preserve, conserve, communicate to the public by telecommunication or on the Internet, loan, distrbute and sell theses worldwide, for commercial or noncommercial purposes, in microform, paper, electronic and/or any other formats.

The author retains copyright ownership and moral rights in this thesis. Neither the thesis nor substantial extracts from it may be printed or otherwise reproduced without the author's permission.
AVIS:

L'auteur a accordé une licence non exclusive permettant à la Bibliothèque et Archives Canada de reproduire, publier, archiver, sauvegarder, conserver, transmettre au public par télécommunication ou par l'Internet, prêter, distribuer et vendre des thèses partout dans le monde, à des fins commerciales ou autres, sur support microforme, papier, électronique et/ou autres formats.

L'auteur conserve la propriété du droit d'auteur et des droits moraux qui protege cette thèse. $\mathrm{Ni}$ la thèse ni des extraits substantiels de celle-ci ne doivent être imprimés ou autrement reproduits sans son autorisation.
In compliance with the Canadian Privacy Act some supporting forms may have been removed from this thesis.

While these forms may be included in the document page count, their removal does not represent any loss of content from the thesis.
Conformément à la loi canadienne sur la protection de la vie privée, quelques formulaires secondaires ont été enlevés de cette thèse.

Bien que ces formulaires aient inclus dans la pagination, il n'y aura aucun contenu manquant. 


\section{Abstract}

In this thesis, we present a Bayesian model selection and parameter estimation scheme for the time domain system identification of a single-degree-of-freedom, nonlinear oscillatory system involving non-trivial fluid-structure interactions using wind-tunnel data. The overall modelling scheme involves three stages: a) selecting a candidate model set; b) estimating their model parameters; c) selecting the optimal model. A backward elimination based stepwise procedure is adopted to form a candidate set whose members are compared using marginal likelihood or evidence calculated using the Chib-Jeliazkov method. The parameter estimation is performed using the Bayesian inference, whereby a Monte Carlo Markov Chain (MCMC) based adaptive Metropolis-Hastings (M-H) algorithm is employed to sample from the joint posterior distribution of the parameters. The Bayesian inference algorithm involves a state estimation problem which is carried out using the Extended Kalman Filter (EKF). To exploit the high performance computing (HPC) platforms, the distributed implementation of the adaptive M-H sampling algorithm is carried out using Message Passing Interface (MPI). The wind tunnel test of a nonlinear aeroelastic oscillator is conducted for validation and application of the proposed modelling scheme. Initially, a numerical study is performed by mimicking the oscillator response from the wind tunnel test to contrast the performance of the model selection via marginal likelihood with asymptotic methods such as Akaike's Information criterion (AIC), Bayesian Information criterion (BIC) and Information Complexity (ICOMP). Using the model selection scheme, the optimal model of the aeroelastic oscillator is identified using the wind-tunnel test. The optimal model can be used to understand and predict the aeroelastic interactions of the coupled system. 
Dedicated to my late grandfather S. Hari Singh Sandhu 
"Life is the art of reaching sufficient conclusions from insufficient data"

[N.K. Sinha and B. Kuszta, Modelling and Identification of Dynamic Systems, 1983]

"Remember that all models are wrong; the practical question is how wrong do they have to be to not be useful."

[G. Box and N. Draper, Empirical Model-Building and Response Surfaces, 1987] 


\section{Acknowledgments}

I would like to thank my supervisors: Prof. Abhijit Sarkar and Prof. Dominique Poirel, for their unwavering support and encouragement throughout the research and the writing of this thesis, my colleague Mohammad Khalil for providing access to his research work, my thesis committee: Prof. David Lau and Prof. Elena Dragomirescu, for their insightful comments and encouragement, and my family for their unconditional love and support throughout my life.

Furthermore, I would like to thank following supercomputer facilities for providing the computational resources needed for this work:

1. "Computations were performed on the Guillimin supercomputer at McGill University and the Colosse supercomputer at Université Laval, under the auspices of Calcul Québec and Compute Canada. The operations of Guillimin and Colosse are funded by the Canada Foundation for Innovation (CFI), the National Science and Engineering Research Council (NSERC), NanoQuébec, and the Fonds Québécois de Recherche sur la Nature et les Technologies (FQRNT)."

2. "Computations were performed on the gpc supercomputer at the SciNet HPC Consortium. SciNet is funded by: the Canada Foundation for Innovation under the auspices of Compute Canada; the Government of Ontario; Ontario Research Fund - Research Excellence; and the University of Toronto."

3. "Computations were performed on a HP Cluster consisting of 176 core cluster with 3.0 GHz Xeon Processors and 704 GB of total memory (RAM) funded by Canada Foundation for Innovation (CFI) and Ontario Innovation Trust (OIT) infrastructure grant for the Canada Research Chair program." 


\section{Citations}

The derivation and the subsequent discussion of the Chib-Jeliazkov method in Chapter 3 is inspired from the following paper:

S. Chib and I. Jeliazkov. Marginal Likelihood From the MetropolisHastings Output. Journal of the American Statistical Association 96(453), 270281. ISSN 01621459 (2001).

The fundamental theory of the Bayesian parameter estimation strategy outlined in Chapter 4 is acquired from the following paper:

M. Khalil, D. Poirel, and A. Sarkar. Probabilistic Parameter Estimation of a Fluttering Aeroelastic System in the Transitional Reynolds Number Regime. Journal of Sound and Vibration (under review) .

The large portion of Chapter 5 including the wind-tunnel set-up and the discussion on the physics of the laminar separation flutter is derived from the following paper:

D. Poirel, Y. Harris, and a. Benaissa. Self-sustained aeroelastic oscillations of a NACA0012 airfoil at low-to-moderate Reynolds numbers. Journal of Fluids and Structures 24(5), 700719. ISSN 08899746 (2008). 


\section{Table of Contents}

$\begin{array}{lll}\text { Abstract } & \text { ii }\end{array}$

Acknowledgments $\quad$ v

$\begin{array}{lll}\text { Citations } & \text { vi }\end{array}$

Table of Contents vii

List of Tables $\quad x$

List of Figures $\quad$ xi

List of Acronyms $\quad$ xv

1 Introduction $\quad 1$

1.1 Motivation . . . . . . . . . . . . . . . . . 1

1.2 Objective $\ldots \ldots \ldots \ldots \ldots \ldots \ldots \ldots \ldots \ldots \ldots \ldots \ldots \ldots \ldots \ldots \ldots$

1.3 Preview of this thesis . . . . . . . . . . . 7

2 Literature Review $\quad 8$

2.1 Model selection . . . . . . . . . . . . . . . . 8

2.1.1 Bayesian model selection . . . . . . . . . . . . . . . 9

2.1.2 Information-theoretic methods . . . . . . . . . . 13

2.2 Parameter estimation . . . . . . . . . . . . . . . . 14

3 Methodology : Model Selection 16

3.1 Introduction . . . . . . . . . . . . . . 16 
3.1 .1 Objective of model selection . . . . . . . . . . 16

3.1.2 Stepwise model selection using Backward elimination . . . . 17

3.1.3 Mathematical preliminaries ............. 19

3.2 Bayesian Model Selection . . . . . . . . . . . . . . . . . 20

3.2.1 Marginal likelihood computation: The Chib-Jeliazkov method 23

3.2.2 Variability of the marginal likelihood estimate . . . . . . . 27

3.2.3 Bayes factor sensitivity on prior specifications . . . . . . 34

3.3 Information-theoretic approach . . . . . . . . . . . 36

4 Methodology: Parameter estimation 39

4.1 State Space Models . . . . . . . . . . . . . . . . . . . . . . 39

4.2 Bayesian Inference . . . . . . . . . . . . . . 41

4.3 State Estimation Using the Extended Kalman Filter . . . . . . . . 43

4.4 Monte Carlo Markov Chain sampling algorithm . . . . . . . . . . . . 49

4.4 .1 Markov Chains . . . . . . . . . . . . 50

4.4.2 Metropolis-Hastings algorithm . . . . . . . . . . . . 52

4.4.3 An Adaptive Random Walk Metropolis algorithm . . . . . . 53

4.5 Maximum Likelihood Estimation . . . . . . . . . . . 59

5 Application: A fluid-structure interaction problem 61

5.1 The nonlinear aeroelastic oscillator . . . . . . . . . . 61

5.1 The wind-tunnel set-up . . . . . . . . . . 61

5.1 .2 The aeroelastic oscillations . . . . . . . . . . . 63

5.1.3 Quasi-steady aerodynamics .............. 67

5.2 Numerical validation $\ldots \ldots \ldots \ldots$. . . . . . . . . 69

5.2 .1 Case 1: Free decay oscillation . . . . . . . 70

5.2 .2 Case 2 : Limit cycle oscillations . . . . . . . . 73

5.2 .3 Important observations . . . . . . . . . . . . 77 
5.3 Modelling of the wind-tunnel data . . . . . . . . . . . . . 81

5.3.1 Free-decay response with no airflow ........... 81

5.3 .2 Laminar separation flutter . . . . . . . . . . . . 84

5.3 .3 Important observations . . . . . . . . . . . . 97

6 Conclusion and future work $\quad 110$

6.1 Conclusion . . . . . . . . . . . . . . . . . 110

6.2 Future work ......................... 111

A Sensitivity analysis : Chib-Jeliazkov method 113

$\begin{array}{ll}\text { B State space conversion through an example } & 119\end{array}$

$\begin{array}{lr}\text { C Gaussian identities } & 129\end{array}$

$\begin{array}{ll}\text { List of References } & 131\end{array}$ 


\section{List of Tables}

$1 \Delta \mathrm{BIC}_{i, j}^{\text {exact }}$ benchmark values for model selection $\ldots \ldots . \ldots . . . .22$

2 Free decay case: $\mathrm{BIC}^{\text {exact }}$ model selection data . . . . . . . . . 71

$3 \quad$ Free decay case: ICOMP model selection data . . . . . . . . . . 72

4 Free decay case: AIC model selection data . . . . . . . . . 72

$5 \quad$ Free decay case BIC model selection data $\ldots \ldots \ldots 73$

6 LCO case: $\mathrm{BIC}^{\text {exact }}$ model selection data . . . . . . . . . . 75

$7 \quad$ LCO case: ICOMP model selection data . . . . . . . . . 75

8 LCO case: AIC model selection data . . . . . . . . . . . . 76

9 LCO case: BIC model selection data $\ldots \ldots \ldots$

10 Updated $\triangle \mathrm{BIC}_{r, G}^{\text {exact }}$ benchmark values for model selection when . . . 80

11 Free decay wind-tunnel data: BIC ${ }^{\text {exact }}$ model selection data . . . . . 83

12 LCO: $\Delta \mathrm{BIC}_{r, G}^{\text {exact }}$ model selection data for $\mathrm{Re}=75,0000 \ldots . . . .90$

13 LCO: $\Delta \mathrm{BIC}_{r, G}^{\mathrm{exact}}$ model selection data for $\mathrm{Re}=85,0000 \ldots \ldots 91$

14 LCO: $\triangle \mathrm{BIC}_{r, G}^{\text {exact }}$ model selection data for $\mathrm{Re}=90,0000 \ldots \ldots 2$

15 LCO: $\triangle \mathrm{BIC}_{r, G}^{\text {exact }}$ model selection data for $\mathrm{Re}=105,0000 \ldots 93$

16 MLE estimate of the coefficient of $M$ in Eq. (103) . . . . . . 108 


\section{List of Figures}

1 Comparison of true response (without the noise) and measured response (with the noise) . . . . . . . . . . . . 2

2 Comparison of response realizations with actual data for model $\mathcal{M}_{1}, \mathcal{M}_{2}$ and $\mathcal{M}_{3}$, in that order, from top to bottom . . . . . . 4

$3 \quad$ Marginal likelihood estimate for varying $w$ and fixed $M \ldots \ldots 30$

$4 \quad$ Estimated integrated autocorrelation for varying $w$ and fixed $\phi^{\star}\left(=\mu_{f}\right) \quad 31$

5 Marginal likelihood estimate for varying estimated integrated autocorrelation time obtained by varying $w$ for fixed $\phi^{\star}\left(=\mu_{f}\right) \ldots 32$

$6 \quad$ Marginal likelihood estimates for varying $M$ and fixed $w \ldots 33$

7 Summary of the EKF procedure . . . . . . . . . . . . . . . . 49

$8 \quad$ Comparison of MCMC chains generated with $w$ values of $0.1 ; 1.0$ and 10.0 (in that order, from top to bottom). All the three chain have same starting position $(\phi=0.0)$ and chain length $(2000) \ldots \ldots 55$

9 Comparison of MCMC chains generated with fixed starting values of

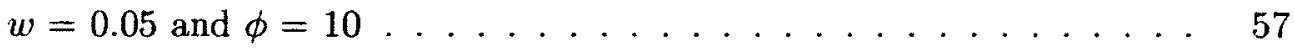

10 Summary of the MCMC procedure untill the burn-in period . . . . . 58

11 Layout of the wind-tunnel . . . . . . . . . . . . 62

12 Schematic of the wing apparatus f............. 62

13 Photograph of the wing apparatus ............. 63

$142 \mathrm{D}$ representation of the wing system .............. 63

15 Measured oscillator response . . . . . . . . . . . . . 65

16 Static aerodynamic moment coefficient about c/4 . . . . . . . 66

17 True and measured free decay responses $\ldots \ldots \ldots$. . . . . . 70 
18 True and measured LCO response . . . . . . . . . . . . . .

19 Comparison of posterior marginal pdfs in the final model (red) and the global model (blue) . . . . . . . . . . . . . . . 78

20 Comparison of response realizations (pink) with the filtered response (blue) $\ldots \ldots \ldots \ldots \ldots \ldots$

21 Free decay case: State estimation results for the final model when $\phi=\hat{\phi} \ldots \ldots \ldots \ldots \ldots \ldots$

22 LCO case: State estimation results for the final model when $\phi=\hat{\phi} \quad . \quad 80$

23 Free decay response with no airflow . . . . . . . . . . . 81

24 Marginal posterior pdfs and MLE estimate (red-dashed line) for the parameters of the final model . . . . . . . . . . . 83

25 Selected joint posterior pdfs for the parameters of the final model . . 83

26 Comparison of marginal pdfs of selected parameters in the final model (red) and the global model (blue) . . . . . . . . . . . . 84

27 Power spectral density (PSD) of the measured oscillator response . . 85

28 The filtered oscillator response used for system identification . . . . . 86

29 Power spectral density (PSD) of the filtered oscillator response . . . . 87

30 Comparison of the measured and filtered LCO response . . . . . . . . 88

31 Marginal posterior pdfs and MLE estimates (red-dashed line) for the parameters of the final model for $\mathrm{Re}=75,000 \ldots \ldots 89$

32 Selected joint posterior pdfs for the parameters of the final model for

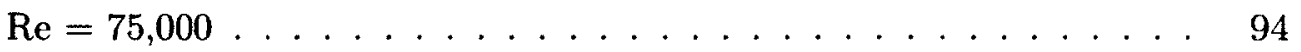

33 Marginal posterior pdfs and MLE estimates (red-dashed line) for the parameters of the final model for $\operatorname{Re}=85,000 \ldots \ldots 9$

34 Selected joint posterior pdfs for the parameters of the final model for

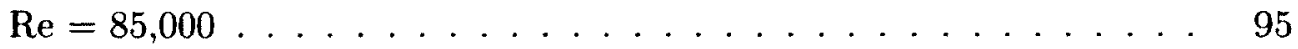


35 Marginal posterior pdfs and MLE estimates (red-dashed line) for the parameters of the final model for $\operatorname{Re}=90,000 \ldots \ldots 9$

36 Selected joint posterior pdfs for the parameters of the final model for

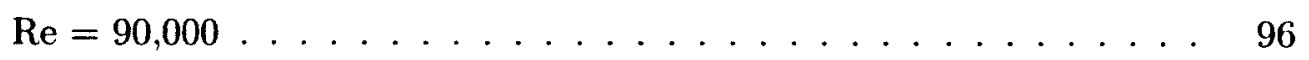

37 Marginal posterior pdfs and MLE estimates (red-dashed line) for the parameters of the final model for $\operatorname{Re}=105,000 \ldots \ldots 6$

38 Selected joint posterior pdfs for the parameters of the final model for

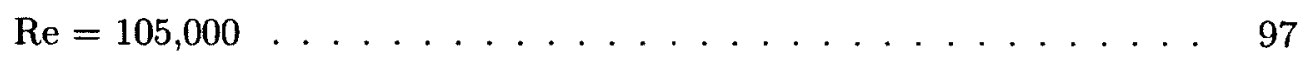

39 Static moment coefficient for $\operatorname{Re}=75,000 \ldots \ldots \ldots$

40 Static moment coefficient for $\operatorname{Re}=85,000 \ldots \ldots 9 . \ldots . \ldots 9$

41 Static moment coefficient for $\operatorname{Re}=90,000$. . . . . . . . . . . . 100

42 Static moment coefficient for $\operatorname{Re}=105,000 \ldots \ldots 100$

43 Static moment coefficient using MLE estimates for different Re values 101

44 Comparison of response realizations obtained using the final chosen model with the filtered response for $\operatorname{Re}=75,000 \ldots \ldots$. . . . . . 102

45 Comparison of response realization obtained using the final chosen model with the filtered response for $\operatorname{Re}=85,000 \ldots \ldots$. . . . . . 103

46 Comparison of response realization obtained using the final chosen model with the filtered response for $\mathrm{Re}=90,000 \ldots \ldots$. . . . . . . 104

47 Comparison of response realization obtained using the final chosen model with the filtered response for $\mathrm{Re}=105,000 \ldots \ldots 105$

48 Wagner's function . . . . . . . . . . . . . . . 106

49 Comparison of the static aerodynamic moment coefficient for the quasisteady and the unsteady $5^{\text {th }}$ order generalized models for $\mathrm{Re}=75,000108$

50 Comparison of the static aerodynamic moment coefficient for the quasisteady and the unsteady $5^{\text {th }}$ order generalized models for $\operatorname{Re}=85,000108$ 
51 Comparison of the static aerodynamic moment coefficient for the quasisteady and the unsteady $5^{\text {th }}$ order generalized models for $\operatorname{Re}=90,000 \quad 109$

52 Comparison of the static aerodynamic moment coefficient for the quasisteady and the unsteady $5^{\text {th }}$ order generalized models for $\operatorname{Re}=105,000109$

53 Marginal likelihood estimates for Bimodal gaussian distribution with modes at -6 and $6 \ldots \ldots \ldots \ldots 114$

54 Marginal likelihood estimates for Bimodal gaussian distribution with modes at -3 and $3 \ldots \ldots \ldots \ldots 115$

55 Marginal likelihood estimates for log-normal distribution without the positivity condition for MCMC samples . . . . . . . . . . . 117

56 Marginal likelihood estimates for log-normal distribution with the positivity condition for MCMC samples . . . . . . . . . . . 118 


\section{List of Acronyms}

\begin{tabular}{ll}
\hline Acronyms & Definition \\
\hline ODE & Ordinary Differential Equation \\
M-H & Metropolis-Hastings \\
RWM & Random Walk Metropolis \\
Re & Reynolds number \\
MLE & Maximum Likelihood Estimation \\
MCMC & Monte Carlo Markov Chain \\
MPI & Message Passing Interface \\
IID & Independent and Identically Distributed \\
pdf & probability density function \\
K-L & Kullback-Leibler \\
TIC & Takeuchi Information Criterion \\
AIC & Akaike's Information Criterion \\
BIC & Bayesian Information Criterion \\
ICOMP & Information Complexity \\
EKF & Extended Kalman Filter \\
AOA & Angle of Attack \\
LCO & Limit Cycle Oscillations \\
\hline
\end{tabular}




\section{Chapter 1}

\section{Introduction}

Understanding the behaviour of dynamical systems using their measured response is one of the primary interests to engineers and scientists. A mathematical model is used to express a complex dynamical system describing an engineering or natural system. This model should be able to capture the underlying physics of the system with a reasonable accuracy for it to be used for prediction, control, health monitoring, performance-based design and various other crucial purposes. The mathematical procedure of building such a model using the measurement data is called system identification. This process is also known as inverse modelling, statistical inference etc., all representing the similar underlying motive. In this thesis, we focus on system identification of a nonlinear dynamical system involving complex fluid-structure interactions.

\subsection{Motivation}

In engineering mechanics, the traditional system identification technique proceeds by proposing a model using the laws of mechanics and then estimating its parameters in the form of a point estimate through some predefined cost function. In this work, 
we wish to solve the two very fundamental issues with this traditional technique: 1) the assumption that the proposed model structure is correctly known a priori can be risky for complex systems, and 2) making inferences entirely based on the point estimates of the model parameters without the consideration of their variability can be deceptive. We demonstrate these arguments in mathematical terms through a simple numerical example.

Consider a quadratic polynomial with an additive Gaussian measurement noise,

$$
y_{i}=a_{0}+a_{1} x_{i}+a_{2} x_{i}^{2}+\epsilon_{i} ; \quad \epsilon_{i} \sim \mathcal{N}\left(0, \sigma^{2}\right)
$$

The parameter values are set as: $a_{0}=1.0, a_{1}=-40.0, a_{2}=60.0$, and $\sigma^{2}=25.0$. The resulting model is termed as the "generating model". A response is generated from the generating model for $x=0.0$ to 1.0 , as shown in Fig. 1. The following three

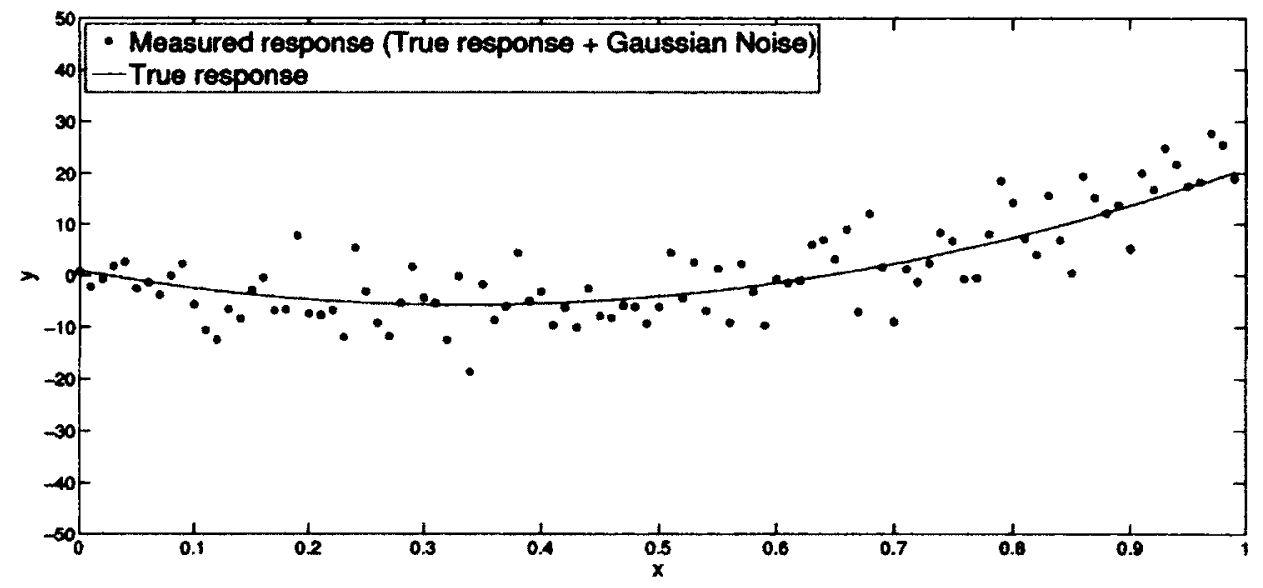

Figure 1: Comparison of true response (without the noise) and measured response (with the noise)

models are proposed to model the measured response:

$$
y_{i}=a_{0}+a_{1} x_{i}+\epsilon_{i} ; \quad \epsilon_{i} \sim \mathcal{N}\left(0, \sigma^{2}\right) \quad \ldots \mathcal{M}_{1}
$$




$$
\begin{array}{rrr}
y_{i}=a_{0}+a_{1} x_{i}+a_{2} x_{i}^{2}+\epsilon_{i} ; & \epsilon_{i} \sim \mathcal{N}\left(0, \sigma^{2}\right) & \ldots \mathcal{M}_{2} \\
y_{i}=a_{0}+a_{1} x_{i}+a_{2} x_{i}^{2}+a_{3} x_{i}^{3}+\epsilon_{i} ; & \epsilon_{i} \sim \mathcal{N}\left(0, \sigma^{2}\right) & \ldots \mathcal{M}_{3}
\end{array}
$$

Using the data from $x=0.0$ to 0.5 , the joint posterior pdf of the static parameters (including $\sigma$ ) of each model is obtained using the Bayesian inference approach detailed in Chapter 4. We use flat priors for all the static parameters for all the three models. The Maximum Likelihood estimation (MLE) estimate, which is same as the mode of the posterior pdf for flat priors, is also obtained. A set of independent samples were drawn from the joint posterior pdf and was used to obtain response realizations from each of the three models. Fig. 2 shows the comparison of the these realizations, response using MLE estimates, the used data $(x=0.0$ to 0.5$)$ and the future data ( $x$ $=0.5$ to 1.0$)$.

The varying fitting and prediction behaviour of the three models in Fig. 2 can be explained through following argument. Since the proposed models strive to compactly represent the information buried in the noisy response while discarding the effect of the noise, the amount of information that a model capture is entirely dependent on the model structure $[1,2]$. Simpler models (like $\mathcal{M}_{1}$ ) tend to extract less information leading to a large bias in the predictions due to the under-fitting, while complex models (like $\mathcal{M}_{3}$ ) tend to capture the noise as a part of the physics leading to a large variance in the predictions due to the over fitting $[1,2]$. This behaviour of the bias and the variance of the predictions as a function of the model complexity is also known as the bias-variance trade-off $[1,2]$.

Hence, both overly simple and overly complex models are inappropriate from the perspective of making predictions or understanding the system dynamics, due to different but equally concerning reasons. Therefore, assuming that the proposed model is correct without the consideration of other plausible models is clearly not a 

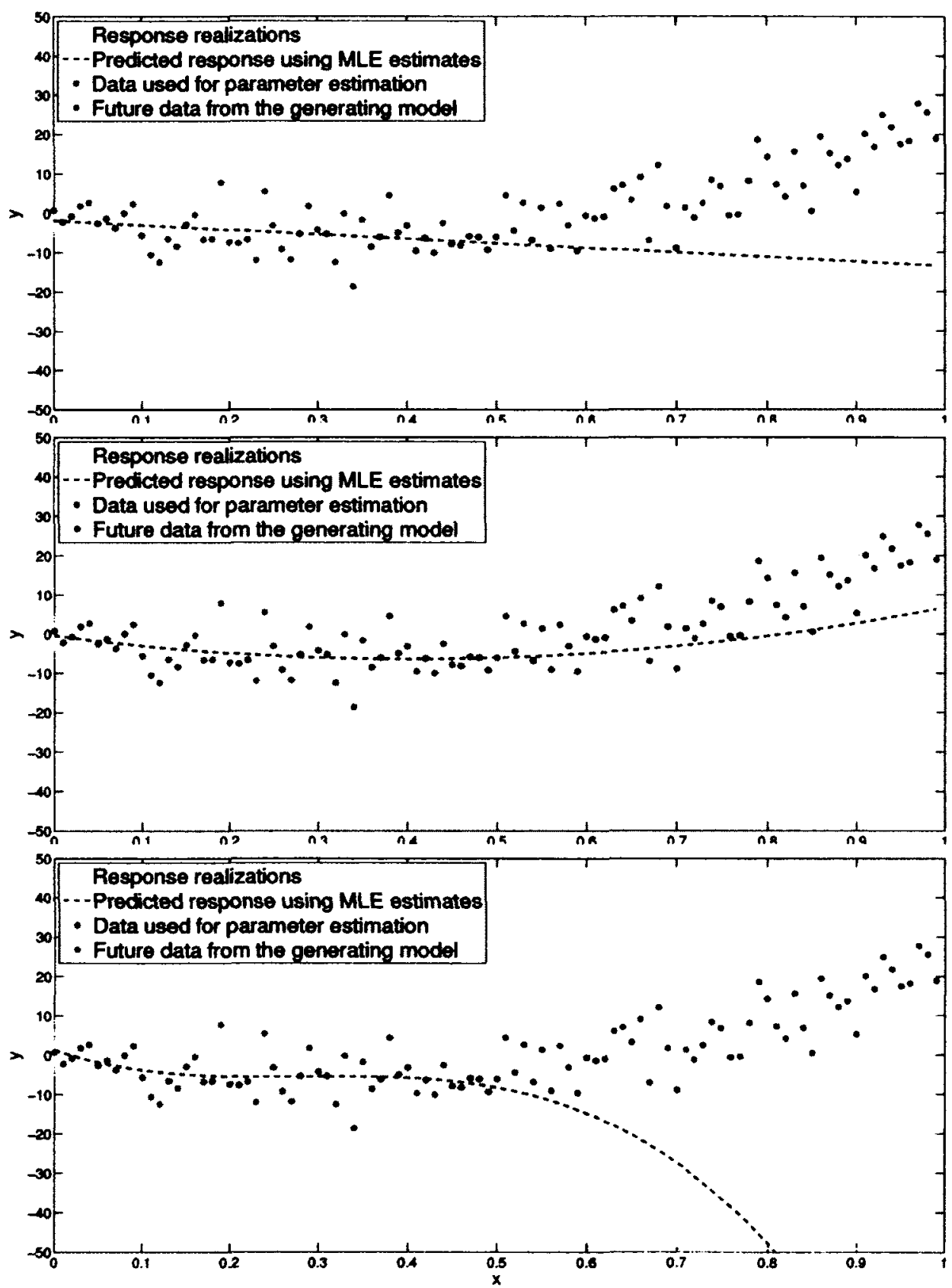

Figure 2: Comparison of response realizations with actual data for model $\mathcal{M}_{1}, \mathcal{M}_{2}$ and $\mathcal{M}_{3}$, in that order, from top to bottom

sensible approach, especially when modeling complex nonlinear systems where very little prior information is available about the system physics. This vagueness about 
the proposed models motivated us to augment the traditional system identification practice with a model selection step.

On similar grounds, the effect of the parameter estimate variability on the predicted response in Fig. 2 can be explained through the following argument. The variability of the parameter estimates is a function of the complexity of the model $[1,2]$. A complex model will have large variability in the parameter estimates resulting in large variability (not centered around MLE response for nonlinear models; for eg. see predicted response of $\mathcal{M}_{3}$ ) of the predicted responses. Therefore, using only the point estimates (like the MLE estimates) can provide incomplete information about the fitting and prediction properties of the proposed models. This behaviour of the inferences based only on the point estimates motivated us to seek a comprehensive Bayesian approach to obtain the joint pdf of the parameter estimates.

\subsection{Objective}

Our objective in this thesis can be described into two categories as follows:

\section{From methodology point of view}

Our aim in regard to developing a comprehensive time-domain based system identification scheme are:

- Model selection: Formulate a general Bayesian model selection scheme to identify a model from a set of plausible models. Also, we aim to contrast its performance with the asymptotic information-theoretic model selection methods through numerical studies.

- Parameter estimation: Formulate a general Bayesian inference scheme to obtain the joint distribution of the static parameters of the model chosen through model 
selection.

We aim to execute both the above tasks by making use of the latest advancements in the field of Monte Carlo sampling in conjunction with the high performance computing (HPC) systems available in Canada.

\section{From application point of view}

In this thesis, we target a nonlinear, single-degree-of-freedom aeroelastic oscillator for validating and implementing the proposed model selection and parameter estimation schemes. The aeroelastic oscillator consists of a NACA0012 wing attached in a windtunnel, performing small amplitude ( $<6$ degrees) limit cycle oscillations (LCO) in the transitional Reynolds number $(\mathrm{Re})$ regime $\left(5.0 \times 10^{4} \leq \mathrm{Re}_{c} \leq 1.3 \times 10^{5}\right)[3-5]$. The oscillations are speculated to be occurring from the nonlinear interactions between the airflow (fluid) and the wing (structure).

Our ultimate aim is to identify the nonlinear physics in these oscillations through a model selection process and quantify this nonlinearity through parameter estimation, using the wind-tunnel experimental data. Before modelling the oscillator data, we wish to identify and quantify the structural properties of the airfoil apparatus by modelling the free decay wind-tunnel data under no airflow. The estimated structural parameters will be used to differentiate the aerodynamic contributions from the overall physics. We wish to perform the overall exercise in two stages:

- Validation: Validate the proposed system identification scheme by using the surrogate data generated through numerical models. We conduct this for two types of responses: 1) free decay, and 2) LCO responses, similar to what is noticed in the wind-tunnel.

- Implementation: Implement the proposed system identification scheme to model 
the measured LCO response from the wind-tunnel for four different Re values. Based on the final chosen models and their estimated parameters, we will study the nature and variation of the aerodynamics of the oscillating system with varying Reynolds number.

\subsection{Preview of this thesis}

- Chapter 2 provides a brief review of the model selection and the parameter estimation schemes.

- Chapter 3 introduces the proposed Bayesian model selection scheme to select the best model among various plausible models.

- Chapter 4 introduces the proposed Bayesian parameter estimation strategy to obtain the joint posterior pdf of the unknown parameters.

- Chapter 5 implements the proposed system identification scheme to model a nonlinear aeroelastic oscillator through wind-tunnel testing.

- Chapter 6 summarizes the findings of this thesis and provides comment on possible future work. 


\section{Chapter 2}

\section{Literature Review}

In this chapter, we review the relevant literature in two sections: 1) model selection methods, and 2) parameter estimation. This concise review is only relevant to the current investigations, but redirects the reader to more exhaustive sources.

\subsection{Model selection}

The model selection methods are statistical approaches that employ, directly or indirectly, the parameter estimates, the model structure and the measured response to confer the suitability of the proposed models $[1,2]$. In this thesis, our goal is to compare the stochastic dynamical models whose static parameters has been estimated in two forms: 1) Frequentist approach: point estimates using the MLE method, and 2) Bayesian: Joint posterior pdf using Bayesian inference. Note that for flat priors, the MLE estimates are same as the mode of the posterior pdf.

In some of the earliest literature in model selection, Jeffreys' [6] advocated the importance of the principle of Ockham's razor in model selection. The Ockham's razor [7-9] is a philosophical principle that states that the simplest model that can explain the data is preferable than the complex ones. It resonates with what Einstein 
once famously said, "Everything should be made as simple as possible, but not simpler". Box and Jenkins [10] also advocated the selection of parsimonious model in the context of time-series analysis. They called it the 'Principle of parsimony'. Inspite of the terminological differences, the Ockham's razor and the principle of parsimony embody the same philosophical idea of model selection that advocates the notion of optimization between the complexity of the model and the fitting of the data. This optimization problem is also known as the Bias-variance trade-off $[1,2]$.

All the available model selection methods can be divided into two categories: 1) Information-theoretic methods (frequentist approach) [1, 2], and 2) Bayesian model selection methods $[11,12]$. The information-theoretic methods employ the MLE estimates while the Bayesian approach employs the posterior pdf to assign a score to every model $[1,2,11,12]$. This score is compared to each of the proposed models to select a best model. More exhaustive comparison of the two model selection approaches can be found in Kadane and Lazar [13], Guyon and Cawley [8] and Zucchini [14].

In this work, we will employ the Bayesian approach due to its generality which avoids approximations based on asymptotic results. Since we will be dealing with a fairly large number of models that have moderate size (roughly 8-25) of parameter vectors, our primary focus is on the computationally efficient implementation of the Bayesian approach by combining the recent advancements in the sampling based statistical methods (to estimate high-dimensional integrals) with the modern HPC systems.

\subsubsection{Bayesian model selection}

Jeffreys [6] is often recognized as a pioneer of applied Bayesian methods. Roberts et al. [15] and Gelman [16] revisit some of the important findings of Jeffreys in 
terms of their relevance and usage in the recent past. The fundamental idea behind the Bayesian model selection is the comparison of the posterior probabilities of the competing models through Bayes factor. Bayes factor is defined as the ratio of the marginal likelihoods (also known as evidence) of the two competing models (See Kass and Raftery [12] and references within for extensive review on Bayes factor). Gull [17] and others $[18,19]$ have shown that the Bayes factor provides a mathematical framework for applying the principle of Ockham's razor or the principle of parsimony in model selection. Inspite of its fundamental significance, Bayes factor suffers from two issues: 1) High sensitivity to the prior distribution of parameters, and 2) high computational cost due to the presence of a high-dimensional integral.

Bayes factor is not robust for relatively non-informative priors to the parameters of the competing models, even for large data sets $[12,13,20-24]$. This behaviour of the Bayes factor is called the Jeffreys-Lindley paradox $[13,25]$. Unlike the posterior distribution, the sensitivity of the Bayes factor gets amplified when increasing the noninformativeness (vagueness) of the proper priors (obtained by increasing the spread of the prior distributions). Three of the most common scenarios where one is prompted to use non-informative proper priors are: 1) No or very little prior information is available, 2) statistical inferences are to be entirely based on the measurement data, and 3) manual assignment of prior rendered impractical by the large number of models being considered. In this work, we face all three scenarios. The only alternative to the manual assignment of a proper prior is the use of improper prior. An improper prior is a prior distribution with unbounded support over the parameter space. However, use of the improper prior results in a Bayes factor that is not well-defined. This behaviour of the Bayes factor with respect to improper priors is called as the 'Bartletts paradox' $[26,27]$.

An extensive literature exists on the automatic Bayesian model selection methods 
that does not require the manual assigning of proper priors, resulting in the Bayes factor that is entirely dependent on the measurement data and the model structure. All these automatic methods involves the usage of improper priors and can be divided into two categories: 1) Methods that convert an improper prior to a proper prior by using some portion of the available data, and 2) use of improper priors that result in a well-defined Bayes factor. Some of the methods in the first category are: 1) Intrinsic Bayes factor (IBF) [28-30], 2) Fractional Bayes factor(FBF) [30-32], 3) Posterior Bayes factor(PBF) [28, 33, 34], and 4) Conventional prior (CP) approach [34]. These methods use some part of the measurement data to convert an improper prior into a proper prior, which is then used to obtain the Bayes factor. However, a prior distribution is naturally defined as representing knowledge before analyzing any type of data from the same system and, therefore, contradicts the Bayesian framework. Over the years, there has been significant progress on the class of improper priors that result in a well-defined Bayes factor. See Strachan and Dijk [26] for a review on all these classes of improper priors.

The second difficulty with the practical implementation of the Bayesian model selection through Bayes factor is the presence of a high dimensional integral over the parameter space. If possible, analytical evaluation of the integral is always preferable due to its accuracy and computational efficiency. However, the analytical methods are only possible for very few specific classes of models and their use is limited in practice for high-dimensional and non-linear models [12].

In practice, the numerical methods are the most commonly used approach to calculate the integral. However, the traditional numerical integration methods like the Monte Carlo integration and the quadrature methods become computationally challenging for high dimensional problems due to the requirement of large sample size for achieving an acceptable accuracy. In recent statistical literature, various 
MCMC methods have been proposed to estimate the integral in its true form. All these methods can be divided into two categories.

The methods in the first category relies on a MCMC sampler that generates a single Markov chain traversing the joint parameter and model space simultaneously [35]. The portion of the time spent by the MCMC sampler in each model is converted into the posterior model probabilities by dividing it by the total time taken by the MCMC sampler. This posterior probability is a normalized version of the evidence. One of the popular method that employ this approach is the Reversible jump MCMC method [36]. This kind of method is generally used in Bayesian model averaging that involves choosing multiple models with a viewpoint of predictions [37-39].

However, there are several drawbacks of the methods that sample from the joint model and parameter space to compute the marginal likelihood. One of the biggest hurdle of these MCMC methods is the tuning of the MCMC sampler to improve the mixing in a high-dimensional joint parameter and model space. The tuning process can be quite time-consuming when comparing non-nested models with complex posterior distributions. Also, all the models that need to be compared should be defined before starting the MCMC sampler. Any new model proposed afterwards will need a completely new MCMC run for all the models, including the new one. This new MCMC run will need a completely new sampler, which has to be tuned again. Therefore, the resulting model comparison process will be computationally challenging, specifically when the model set changes along the model selection procedure.

The second category of MCMC methods compute the integral for each model separately by using the MCMC sampling output of their posterior distributions. Han and Carlin [35] provides a comparative review of the MCMC based methods for the joint space search and individual calculation techniques with an example. Various methods have been proposed depending on the MCMC algorithm used to generate 
the posterior samples (See Chib [40] and Chib and Jeliazkov [41] for methods in this category).

\subsubsection{Information-theoretic methods}

The information-theoretic approach of model selection operates by striking a balance between the measure of fit and the complexity of the model $[1,2]$. The complex models are penalized through a penalty term that acts against the measure of fit term leading to an optimization type issue across the proposed model set. Depending on the cost function employed to obtain the point estimate, various information theoretic approaches exist in the literature (see the book by Konishi and Kitagawa [1] for comprehensive review on the information-theoretic methods). We will limit our review to the methods pertaining to the MLE method.

Akaike's Information Criterion (AIC) [42] and Bayesian Information Criterion (BIC) [43] are the two most widely used information-theoretic methods. The AIC is derived using the concept of Kullback-Leibler information under the assumption that the model exist very near to the true model (for derivation see Konishi [1]). The final expression for AIC is $[1,2]$

$$
\mathrm{AIC}=-2(\text { maximum } \log \text {-likelihood })+2 \text { (number of parameters })
$$

The model with minimum value of AIC is chosen as the best model. AIC is also shown to have a Bayesian justification under the assumption that the information in prior is comparable to the information provided by the data [1]. However, this is rarely the case in practice as prior information is often limited and conflicting with the information provided by the data.

On the other hand, $\mathrm{BIC}$ is derived by approximating the integral in Bayes factor 
by Laplace's method with an assumption that the likelihood function is highly peaked around the MLE estimate, which is usually the case for large data sample cases $[1,44]$. The ambiguity attached to the definition of "Large data" that leads to BIC is often questioned in the statistical literature [44] (for full derivation see Konishi [1]). The final expression for $\mathrm{BIC}$ is $[1,2]$

$$
\begin{aligned}
\mathrm{BIC}= & -2(\text { maximum } \log \text {-likelihood }) \\
& +2(\text { number of parameters }) \times(\text { logarithm of the size of data vector })
\end{aligned}
$$

Notice that $\mathrm{AIC}$ and $\mathrm{BIC}$ differ in their penalty terms. The BIC is adding higher penalty to models with higher terms than the AIC. Due to this difference, BIC has been shown to select simpler models as the best model than AIC. See Konishi [1], Burnham [45] and Koehler and Murphree [46] for the comparison of AIC and BIC through numerical examples. Also see Smith and Spiegelhalter [47] for the comparison of Bayes factor with AIC and BIC. Additionally, refer to Berger [11] for the comparison of the Conventional prior approach, BIC and Intrinsic and fractional Bayes factor. Recently, a new criterion called Information Complexity (ICOMP) [48] was proposed whose penalty term also includes the correlation structure of the parameters rather than just the number of parameters (as in $\mathrm{AIC}$ and $\mathrm{BIC}$ ) to measure the complexity of the model.

\subsection{Parameter estimation}

Once a stochastic model and a noisy measurement data is available, its unknown or incompletely known static parameters can be estimated by using the measured response through a statistical inversion process. This estimation task is popularly known as parameter estimation [49-51]. All the statistical methods that are used in 
performing parameter estimation can be divided in two categories: 1) Frequentist, and 2) Bayesian [52-54]. The two categories differ in two ways: 1) the probabilistic interpretation of what constitutes the 'uncertainty', and 2) inclusion of the prior knowledge.

The frequentist parameter estimation approach is the classical approach which considers the unknown static parameters as fixed, whose point estimate is obtained by minimizing a predefined loss function of the measured response and the model structure. One of the widely used method in this category is the Maximum Likelihood Estimation (MLE) method $[1,2,55]$, where the value of static parameters that make the data as likely as possible is chosen.

On the other hand, the Bayesian approach treats the unknown parameters as random variables whose joint probability density function (pdf) is obtained using the Bayes theorem [52, 56-58]. Unlike any frequentist method, the Bayesian inference also provides the variability on the point estimates through a mathematical framework that also allows for the inclusion of the prior knowledge, if any, about the parameters. Beck and Katafygiotis [59-61] pioneered the application of Bayesian system identification techniques to model nonlinear dynamical models by using input-output data. Although the frequentist approach still remains a widely popular approach due to its mathematical simplicity and practicality in its numerical implementation, the Bayesian approach is slowly gaining popularity due to its statistically rigor approach and generality.

In this work, we use Bayesian inference to obtain the joint posterior pdf of model parameters. The Bayesian approach employed in this work is the extension of an existing implementation of the Bayesian inference [5]. The MLE estimates are available as a by-product of the numerical implementation (See Khalil [5] for references within). 


\section{Chapter 3}

\section{Methodology : Model Selection}

In this chapter, we will discuss model selection methods with a focus on Bayesian model selection. First, we will discuss the objective of the model selection methods. Then, a backward elimination based stepwise model selection scheme is described. Consequently, Bayesian model selection is discussed in detail with a focus on the ChibJeliazkov method of computing the marginal likelihood. At the end, the Informationtheoretic approach of model selection is briefly introduced.

\subsection{Introduction}

\subsubsection{Objective of model selection}

In general, the objective of model selection methods is a debatable issue in the statistical literature $[1,2]$. It is sometimes mistakenly perceived that the model selection methods aim to uncover the true model by using the measurement data. However, no mathematical model exists which can exactly represent a physical phenomenon.

Since reality is an infinite dimensional $[1,2]$, the dimension of the hypothetical true mathematical model will also be infinite. Therefore, the estimation of an infinite dimensional model is not practically feasible using finite set of the measurement data 
containing only limited information about the system.

On the other hand, a mathematical model, by definition, is an approximate tool to represent an infinitely dimensional system in an objective way in order to make inference (prediction and understanding) $[1,2]$. This definition renders the term "true model" an oxymoron. In other words, the true model is a purely theoretical concept and does not exists in practice.

In this chapter, our objective is to identify the best approximating mathematical model from a set of models by using the measurement data through model selection procedure. Although the best model identified using model selection methods will not represent the reality, it is believed to act as a suitable tool to extract significant information about the system dynamics and make meaningful predictions about the phenomenon at hand $[1,2]$.

The success of the model selection method critically hinges on the appropriate choice of the initial model set. Hence, if the models in the candidate model set are bad, the least bad model will be selected. Next, we discuss the model proposing scheme used in this thesis.

\subsubsection{Stepwise model selection using Backward elimination}

Critical to any model selection effort is the judicious selection of the candidate model set. The larger the size of the candidate model, the higher the chances of identifying the most appropriate model. However, the optimal size of the candidate model set is limited by the time and computational resources available to the modeler.

In this thesis, we resort to a backward elimination based stepwise model selection scheme $[62,63]$. According to this approach, a global model is initially selected such that it comprehensively captures the relevant physical characteristics of the system. In the context of stochastic ordinary differential equation (ODE) models, the global 
model is assumed to have $N$ additive terms other than the model noise term. Each of the $N$ terms is characterized by a unique static parameter. The ideal approach of constructing the candidate model would be to include all the nested models [2] derived from the global model by eliminating various terms.

However, this approach of considering all the possible nested models for model selection suffers from a computational efficiency problem as follows. For a global model having $N$ terms, the number of such nested models is $\sum_{n=1}^{N}{ }^{N} C_{n}$. For instance, a global model consisting of $N=12$ terms, the number of the nested models is 4094 . Clearly, it is not practical to include all these nested models in the candidate model set.

Thus, we adopt the following hierarchical approach to generate the candidate model set and then the selection of the optimal model. Initially, $N$ nested reduced models, each containing $(N-1)$ terms or static parameters, are constructed for the global model by removing a single term, one at a time. These $N$ reduced models combined with the global model constitute the initial candidate model set. The model selection is performed on this candidate model set to select an optimal model, which is then designated as the new global model. This approach of stepwise term reduction and model selection is repeated on the new global model.

In other words, if the current global model is denoted as $\mathcal{M}_{G}$ having $N$ terms, any model selection step will involve selecting of an optimal model from a candidate model set $\left\{\left\{\mathcal{M}_{1}, \mathcal{M}_{2}, \ldots, \mathcal{M}_{r}, \ldots, \mathcal{M}_{N}\right\},\left\{\mathcal{M}_{G}\right\}\right\}$, where $\mathcal{M}_{r}$ 's are the nested reduced models having one term less than the global model $\mathcal{M}_{G}$. Note that we are excluding the model noise term in the term reduction exercise in order to maintain the stochastic nature of the inverse modelling problem.

The stepwise model selection process continues until the optimal model selected is the current global model $\mathcal{M}_{G}$ itself. This stopping criterion means that the removal 
of any term from the current global model will lead to a underfitted model and the current global model is the final optimal model.

We would also like to state that this approach of term reduction and model selection might not result in the best model nested in the initially proposed global model $[64-66]$. However, this approach is practical when the knowledge of system is limited and the dimension of the initial global model is too large to include all the possible nested models in the candidate model set.

\subsubsection{Mathematical preliminaries}

We assume that the noisy measurement data $\mathbf{d}$ has been acquired and a candidate model set $\left\{\mathcal{M}_{1}, \mathcal{M}_{2}, \ldots, \mathcal{M}_{i}, \ldots, \mathcal{M}_{P}\right\}$ has been proposed to model the data $\mathbf{d}$. Each model $\mathcal{M}_{i}$ is characterized by a likelihood function $\mathrm{p}_{i}\left(\mathbf{d} \mid \boldsymbol{\phi}_{i}\right)$ and a prior pdf $\mathrm{p}_{i}\left(\phi_{i}\right)$, where $\phi_{i}$ is the static parameter vector of size $p_{i}$. We further assume that the posterior pdf $\mathrm{p}\left(\phi_{i} \mid \mathbf{d}\right)$ has been obtained using the Bayesian inference equation $[5,58]$

$$
\mathrm{p}_{i}\left(\phi_{i} \mid \mathbf{d}\right)=\frac{\mathrm{p}_{i}\left(\mathbf{d} \mid \phi_{i}\right) \mathrm{p}_{i}\left(\phi_{i}\right)}{\mathrm{p}_{i}(\mathbf{d})}
$$

where $\mathrm{p}_{i}(\mathrm{~d})=\int \mathrm{p}_{i}\left(\mathrm{~d} \mid \phi_{i}\right) \mathrm{p}_{i}\left(\phi_{i}\right) d \phi_{i}$ is just a normalizing constant, also called the marginal likelihood. Note that $\mathrm{p}_{i}\left(\phi_{i} \mid \mathbf{d}\right)$ represents $\mathrm{p}\left(\phi_{i} \mid \mathbf{d}, \mathcal{M}_{i}\right)$. We assume that the posterior pdf $\mathrm{p}_{i}\left(\phi_{i} \mid \mathbf{d}\right)$ in Eq. (7) is available in the form of a finite sample set $\left\{\phi_{i}^{(1)}\right.$, $\left.\phi_{i}^{(2)}, \ldots, \phi_{i}^{(g)}, \ldots, \phi_{i}^{(M)}\right\}$ through the parameter estimation scheme discussed in Chapter 4 . The mode of the posterior $p_{i}\left(\phi_{i} \mid \mathbf{d}\right)$ is also available through the optimization scheme discussed in Chapter 4. For flat priors $\left(\mathrm{p}_{i}\left(\phi_{i}\right) \propto 1\right)$, the mode of the posterior is the MLE estimate $\hat{\phi}_{i}$. 


\subsection{Bayesian Model Selection}

Bayesian model selection methods exploit the Bayes theorem to infer the suitability of every proposed model $\mathcal{M}_{i}$ in terms of its posterior probability $\mathrm{p}_{i}\left(\mathcal{M}_{i} \mid \mathbf{d}\right)$ of representing the data $\mathrm{d}$ as $[11,12]$

$$
\mathrm{p}_{i}\left(\mathcal{M}_{i} \mid \mathbf{d}\right)=\frac{\mathrm{p}_{i}(\mathbf{d}) \mathrm{p}_{i}\left(\mathcal{M}_{i}\right)}{\sum_{s=1}^{P} \mathrm{p}_{s}(\mathbf{d}) \mathrm{p}_{s}\left(\mathcal{M}_{s}\right)}
$$

where $\mathrm{p}_{i}\left(\mathcal{M}_{i}\right)$ is the prior pdf of model $\mathcal{M}_{i}$ and $\mathrm{p}_{i}(\mathrm{~d})$ is the marginal likelihood of the data $\mathbf{d}$. Note that $\mathrm{p}_{i}(\mathbf{d})$ denotes $\mathrm{p}\left(\mathbf{d} \mid \mathcal{M}_{i}\right)$. In other words, the posterior probability $\mathrm{p}_{i}\left(\mathcal{M}_{i} \mid \mathbf{d}\right)$ is obtained by updating the prior $\operatorname{pdf} \mathrm{p}_{i}\left(\mathcal{M}_{i}\right)$ with the information provided by the data $\mathbf{d}$ through the marginal likelihood $\mathrm{p}_{i}(\mathbf{d})$. For the same reason, the marginal likelihood $\mathrm{p}_{i}(\mathbf{d})$ is also stated as the evidence provided by the data $\mathrm{d}$ in the favor of the model $\mathcal{M}_{i}$.

The posterior pdf $\mathrm{p}\left(\mathcal{M}_{i} \mid \mathbf{d}\right)$ in Eq. (8) probabilistically represents that $\mathcal{M}_{i}$ has generated the data $d$. Hence, the model with the largest posterior probability (obtained from $p\left(M_{i} \mid \mathbf{d}\right)$ ) will represent the data $\mathbf{d}$ more precisely than any other model in the candidate model set. Since the denominator in Eq. (8) is a constant for a given candidate model set, the numerator $p_{i}(d) p_{i}\left(\mathcal{M}_{i}\right)$ identifies the best model. Further assuming that all the models are equally likely before analyzing the data, $\mathrm{p}_{i}\left(\mathcal{M}_{i}\right) \propto 1$, model selection can then be performed by computing the marginal likelihood $\mathrm{p}_{i}(\mathbf{d})$ of the models. This methodology forms the basis of the Bayesian model selection.

The assumption of equally probable models in Eq. (8) is justified in the circumstances where only a limited information is available about the system before analyzing the data d. Assigning equal prior probabilities to all the proposed models will also ensure that only the data $\mathbf{d}$ will dictate the model selection process, with no 
interference from subjective, and often ill-advised, prior knowledge of the modeler. This assumption is followed throughout this thesis.

In practice, an important quantity of $-2 \log p_{i}(d)$ is used to carry out the Bayesian model selection [1]. This quantity is also called as the exact version of Bayesian Information Criterion, represented as $\mathrm{BIC}_{i}^{\text {exact }}$, after a more commonly used asymptotic version $\mathrm{BIC}_{i}[1]$. For model $\mathcal{M}_{i}$,

$$
\mathrm{BIC}_{i}^{\text {exact }}=-2 \log \mathrm{p}_{i}(\mathbf{d})=-2 \log \left\{\int \mathrm{p}_{i}\left(\mathbf{d} \mid \phi_{i}\right) \mathrm{p}_{i}\left(\phi_{i}\right) d \phi_{i}\right\}
$$

When comparing two models $\mathcal{M}_{i}$ and $\mathcal{M}_{j}$, the difference in $\mathrm{BIC}^{\text {exact }}$ values is calculated to obtain $\triangle \mathrm{BIC}_{i, j}^{\text {exact }}$ as

$$
\begin{aligned}
\Delta \mathrm{BIC}_{i, j}^{\text {exact }} & =\mathrm{BIC}_{i}^{\text {exact }}-\mathrm{BIC}_{j}^{\text {exact }} \\
& =-2\left[\log \mathrm{p}_{i}(\mathbf{d})-\log \mathrm{p}_{j}(\mathbf{d})\right] \\
& =-2 \log \left\{\frac{\mathrm{p}_{i}(\mathbf{d})}{\mathrm{p}_{j}(\mathbf{d})}\right\}=-2 \log \left\{\frac{\int \mathrm{p}_{i}\left(\mathbf{d} \mid \phi_{i}\right) \mathrm{p}_{i}\left(\phi_{r}\right) d \phi_{i}}{\int \mathrm{p}_{j}\left(\mathbf{d} \mid \phi_{j}\right) \mathrm{p}_{j}\left(\phi_{j}\right) d \phi_{j}}\right\} \\
& =-2 \log \mathrm{BF}_{i, j}
\end{aligned}
$$

Here, $\mathrm{BF}_{i, j}$ is the commonly known Bayes factor [12]. While the Bayes factor is commonly used for pair-wise hypothesis testing, $\mathrm{BIC}^{\text {exact }}$ is a practically efficient tool to execute the model selection task on large number of models. Also, BIC ${ }^{\text {exact }}$ shares the same scale as the information-theoretic model selection methods, which makes the comparison study of the both genres a relatively insightful exercise.

Initially proposed by Jeffreys [6] and later modified by Kass and Raftery [12], Table 1 shows the benchmark values of $\Delta \mathrm{BIC}_{i, j}^{\text {exact }}$ for selecting between $\mathcal{M}_{i}$ and $\mathcal{M}_{j}$. As mentioned by Kass and Raftery [12], the interpretation of Table 1 depends on the context of the model structure and, therefore, only seems to furnish approximate 
guidelines. Also, as discussed later in this chapter, the use of improper prior with well behaved Bayes factor might result in the change of the order of the values shown in Table 1. Therefore, we will revisit the $\Delta \mathrm{BIC}_{i, j}^{\text {exact }}$ benchmark values in Table 1 through a numerical study, performed in the context of nonlinear dynamical systems and the data type being considered in this thesis.

\begin{tabular}{|c|l|}
\hline$\Delta \mathrm{BIC}_{i, j}^{\text {exact }}$ & Evidence against $\mathcal{M}_{i}$ \\
\hline$<2$ & Bare minimum \\
2 to 6 & Positive \\
6 to 10 & Strong \\
$>10$ & Very strong \\
\hline
\end{tabular}

Table 1: $\Delta \mathrm{BIC}_{i, j}^{\text {exact }}$ benchmark values for model selection

The model selection performed using the Bayesian approach $\left(\mathrm{BIC}^{\text {exact }}\right.$ or Bayes factor) has many striking properties. Few of them are listed below:

- The Bayesian approach is consistent in selecting the true model as the optimal model, provided that the true model is present in the proposed model set $[1,11]$. This 'nice' property is practically desirable beside the fact that the true model is never known.

- The Bayesian approach provides a mathematical framework to implement the principle of Ockham's razor [7-9] and the principle of parsimony [10] which state that out of all the models that fit the data comparably, the simplest of all should be chosen. This inherited and natural property of the Bayesian approach makes it highly advantageous over the objective model selection approaches where the idea of penalizing complex models is mathematically doubted to this date $[7,13]$.

- The Bayesian approach holds no assumption about the kind or quantity of models or the type of posterior distributions or the size and type of data that 
can be considered $[11,12]$. This property has led to the widespread practical desirability of the approach.

In-spite of the striking properties, performing model selection though $\Delta \mathrm{BIC}_{i, j}^{\text {exact }}$ suffers from two implementation hurdles under the given context. One, for a large sized $\phi_{i}$, the computation of the marginal likelihood $\mathrm{p}_{i}\left(\phi_{i}\right)$ in Eq. (9) involves a high dimensional integral that needs to be evaluated. Second, for very little prior knowledge about the system, the assigning of the prior $\operatorname{pdf}_{i}\left(\phi_{i}\right)$ can be problematic due to the sensitivity of model selection results on the relative prior specifications of the models being compared. These two issues are discussed in detail.

\subsubsection{Marginal likelihood computation : The Chib-Jeliazkov method}

The computation of marginal likelihood $p_{i}(d)$ through Eq. (9) involves an integral of dimension equal to the size of the vector $\phi_{i}$. Untill recently, the exact calculation of the marginal likelihood was often avoided for the sake of practical simplification, by taking recourse to the asymptotic objective model selection methods. As the MCMC and Bayesian literature advanced, several numerical methods were proposed to obtain an estimate of the marginal likelihood using the MCMC samples generated from the posterior distribution $[35,40,67]$.

In this thesis, we implement the Chib-Jeliazkov method [40] to obtain an estimate of the marginal likelihood by using the MCMC samples generated from the posterior distribution. The Chib-Jeliazkov method is designed for cases where the MCMC samples are being generated by the Metropolis-Hastings algorithm [68]. In this thesis, we generate the posterior samples through an adaptive, one-block $\mathrm{M}$ - $\mathrm{H}$ sampling algorithm outlined in Chapter 4. The mathematical formulation shown here is derived 
from Chib [40] for the case of one-block sampling.

We remove the model index $i$ in this section for the sake of simplicity in mathematical exposition. Consider the Bayesian inference equation for obtaining the joint posterior pdf $\mathrm{p}(\phi \mid \mathbf{d})$,

$$
\mathrm{p}(\boldsymbol{\phi} \mid \mathbf{d})=\frac{\mathrm{p}(\mathbf{d} \mid \phi) \mathrm{p}(\phi)}{\mathrm{p}(\mathbf{d})}
$$

where

$$
\mathrm{p}(\mathbf{d})=\int \mathrm{p}(\mathbf{d} \mid \boldsymbol{\phi}) \mathrm{p}(\boldsymbol{\phi}) d \boldsymbol{\phi}
$$

Re-arranging and taking logarithm on both sides of Eq. (11), we get

$$
\log \mathbf{p}(\mathbf{d})=\log \mathbf{p}(\mathbf{d} \mid \boldsymbol{\phi})+\log \mathbf{p}(\boldsymbol{\phi})-\log \mathrm{p}(\boldsymbol{\phi} \mid \mathbf{d})
$$

The marginal likelihood $\mathrm{p}(\mathbf{d})$ is a constant for a given data set $\mathbf{d}$ and does not depend on the value of $\phi$. Using this property and evaluating Eq. (13) at some preselected point $\phi^{*}$ in the posterior space, we get

$$
\log \mathrm{p}(\mathbf{d})=\log \mathrm{p}\left(\mathbf{d} \mid \boldsymbol{\phi}^{*}\right)+\log \mathrm{p}\left(\boldsymbol{\phi}^{*}\right)-\log \mathrm{p}\left(\boldsymbol{\phi}^{*} \mid \mathbf{d}\right)
$$

Since, the likelihood function $\mathrm{p}(\mathrm{d} \mid \phi)$ and the prior $\operatorname{pdf} \mathrm{p}(\phi)$ are known quantities, the values of $\mathrm{p}\left(\mathrm{d} \mid \phi^{*}\right)$ and $\mathrm{p}\left(\phi^{*}\right)$ are readily available. The only term unknown on the right-hand side of Eq. (14) is the posterior ordinate $p\left(\phi^{*} \mid \mathbf{d}\right)$. If an estimate $\hat{p}\left(\phi^{*} \mid \mathbf{d}\right)$ of the posterior ordinate $\mathrm{p}\left(\phi^{*} \mid \mathbf{d}\right)$ can be obtained by using the posterior samples $\phi^{(g)}$, an estimate of the marginal likelihood can be obtained using Eq. (14) as

$$
\log \hat{\mathbf{p}}(\mathbf{d})=\log \mathbf{p}\left(\mathbf{d} \mid \boldsymbol{\phi}^{*}\right)+\log \mathbf{p}\left(\boldsymbol{\phi}^{*}\right)-\log \hat{\mathbf{p}}\left(\boldsymbol{\phi}^{*} \mid \mathbf{d}\right)
$$

Hence, the problem of estimating marginal likelihood is reduced to obtaining $\hat{p}\left(\phi^{*} \mid \mathbf{d}\right)$. 
Next, we assume that total $M$ posterior samples $\left\{\phi^{(1)}, \phi^{(2)}, \ldots \phi^{(g)} \ldots, \phi^{(M)}\right\}$ have been generated through a M-H algorithm with a fixed proposal density $q\left(\phi, \phi^{\prime}\right)$. The proposal density $q\left(\phi, \phi^{\prime}\right)$ governs the proposed transition of the Markov chain from the current position $\phi$ to the new position $\phi^{\prime}$. According to the $\mathrm{M}-\mathrm{H}$ algorithm, the probability $\alpha\left(\phi, \phi^{\prime} \mid \mathbf{d}\right)$ that the proposed move $\phi^{\prime}$ is accepted is [68]

$$
\alpha\left(\boldsymbol{\phi}, \boldsymbol{\phi}^{\prime} \mid \mathbf{d}\right)=\min \left(1, \frac{\mathrm{p}\left(\boldsymbol{\phi}^{\prime} \mid \mathbf{d}\right)}{\mathrm{p}(\boldsymbol{\phi} \mid \mathbf{d})} \frac{q\left(\boldsymbol{\phi}^{\prime}, \boldsymbol{\phi}\right)}{q\left(\boldsymbol{\phi}, \boldsymbol{\phi}^{\prime}\right)}\right)
$$

The net probability $\mathrm{p}\left(\phi, \phi^{\prime} \mid \mathrm{d}\right)$, also called the transition kernel, with which the Markov chain will transition from $\phi$ to $\phi^{\prime}$ can be written as

$$
\mathrm{p}\left(\boldsymbol{\phi}, \boldsymbol{\phi}^{\prime} \mid \mathbf{d}\right)=\alpha\left(\boldsymbol{\phi}, \boldsymbol{\phi}^{\prime} \mid \mathbf{d}\right) q\left(\boldsymbol{\phi}, \boldsymbol{\phi}^{\prime}\right)
$$

From the reversibility property of the transition kernel of the M-H algorithm [68],

$$
\mathrm{p}\left(\boldsymbol{\phi}, \boldsymbol{\phi}^{\prime} \mid \mathbf{d}\right) p(\boldsymbol{\phi} \mid \mathbf{d})=p\left(\boldsymbol{\phi}, \phi^{\prime} \mid \mathbf{d}\right) p\left(\phi^{\prime} \mid \mathbf{d}\right)
$$

Since Eq. (18) is true for any point $\phi^{\prime}$, substituting it by $\phi^{*}$ and using Eq. (17), we get

$$
\int \alpha\left(\boldsymbol{\phi}^{*}, \boldsymbol{\phi} \mid \mathbf{d}\right) q\left(\boldsymbol{\phi}^{*}, \boldsymbol{\phi}\right) \mathbf{p}\left(\boldsymbol{\phi}^{*} \mid \mathbf{d}\right) d \boldsymbol{\phi}=\int \alpha\left(\boldsymbol{\phi}, \boldsymbol{\phi}^{*} \mid \mathbf{d}\right) q\left(\boldsymbol{\phi}, \boldsymbol{\phi}^{*}\right) \mathbf{p}(\boldsymbol{\phi} \mid \mathbf{d}) d \boldsymbol{\phi}
$$

Since the posterior ordinate $\mathrm{p}\left(\phi^{*} \mid \mathbf{d}\right)$ is just a constant, taking it out of the integral and re-arranging, we get

$$
\mathrm{p}\left(\phi^{*} \mid \mathbf{d}\right)=\frac{\int \alpha\left(\phi, \phi^{*} \mid \mathbf{d}\right) q\left(\phi, \phi^{*}\right) \mathrm{p}(\boldsymbol{\phi} \mid \mathbf{d}) d \boldsymbol{\phi}}{\int \alpha\left(\phi^{*}, \boldsymbol{\phi} \mid \mathbf{d}\right) q\left(\phi^{*}, \phi\right) d \phi}
$$

where the integral in the numerator is w.r.t. $\phi$ from the posterior $p(\phi \mid d)$ and the 
one in the denominator is w.r.t. $\phi$ from the fixed pdf $q\left(\phi^{*}, \phi\right)$. We generate samples $\left\{\phi^{(1)}, \phi^{(2)}, \ldots \phi^{(j)} \ldots, \phi^{(J)}\right\}$ from the pdf $q\left(\phi^{*}, \phi\right)$ through Monte Carlo sampling [69]. Using the samples $\phi^{(g)}$ and $\phi^{(j)}$, the posterior pdf $\mathrm{p}(\phi \mid \mathbf{d})$ and the pdf $q\left(\phi^{*}, \phi\right)$ can be represented in a discrete form as

$$
\mathrm{p}(\boldsymbol{\phi} \mid \mathbf{d})=\frac{1}{M} \sum_{g=1}^{M} \delta\left(\phi-\phi^{(g)}\right) ; q\left(\phi^{*}, \phi\right)=\frac{1}{J} \sum_{j=1}^{J} \delta\left(\phi-\phi^{(j)}\right)
$$

Substituting these discrete versions of $\mathrm{p}(\phi \mid \mathbf{d})$ and $q\left(\phi^{*}, \phi\right)$ in Eq. (75), we get

$$
\begin{aligned}
& \hat{\mathrm{p}}\left(\boldsymbol{\phi}^{*} \mid \mathbf{d}\right)=\frac{\int \alpha\left(\boldsymbol{\phi}, \boldsymbol{\phi}^{*} \mid \mathbf{d}\right) q\left(\boldsymbol{\phi}, \boldsymbol{\phi}^{*}\right)\left\{\frac{1}{M} \sum_{g=1}^{M} \delta\left(\phi-\phi^{(g)}\right)\right\} d \phi}{\int \alpha\left(\phi^{*}, \boldsymbol{\phi} \mid \mathbf{d}\right)\left\{\frac{1}{J} \sum_{j=1}^{J} \delta\left(\phi-\phi^{(j)}\right)\right\} d \phi} \\
& =\frac{\frac{1}{M} \sum_{g=1}^{M} \int \alpha\left(\boldsymbol{\phi}, \phi^{*} \mid \mathbf{d}\right) q\left(\phi, \phi^{*}\right) \delta\left(\phi-\phi^{(g)}\right) d \phi}{\frac{1}{J} \sum_{j=1}^{J} \int \alpha\left(\phi^{*}, \phi \mid \mathbf{d}\right) \delta\left(\phi-\phi^{(j)}\right) d \phi} \\
& =\frac{\frac{1}{M} \sum_{g=1}^{M} \alpha\left(\phi^{(g)}, \phi^{*} \mid \mathbf{d}\right) q\left(\phi^{(g)}, \phi^{*}\right)}{\frac{1}{J} \sum_{j=1}^{J} \alpha\left(\phi^{*}, \phi^{(j)} \mid \mathbf{d}\right)} .
\end{aligned}
$$

Substituting $\alpha\left(\phi^{*}, \phi \mid \mathbf{d}\right)$ from Eq. (19),

$$
\hat{\mathrm{p}}\left(\boldsymbol{\phi}^{*} \mid \mathbf{d}\right)=\frac{\frac{1}{M} \sum_{g=1}^{M} \operatorname{Min}\left(1, \frac{\mathrm{p}\left(\phi^{*} \mid \mathbf{d}\right)}{\mathrm{p}\left(\phi^{(g)} \mid \mathbf{d}\right)} \frac{q\left(\phi^{*}, \phi^{(g)}\right)}{q\left(\phi^{(g)}, \phi^{*}\right)}\right) q\left(\phi^{(g)}, \phi^{*}\right)}{\frac{1}{J} \sum_{j=1}^{J} \operatorname{Min}\left(1, \frac{\mathrm{p}\left(\phi^{(j)} \mid \mathbf{d}\right)}{\mathrm{p}\left(\phi^{*} \mid \mathbf{d}\right)} \frac{q\left(\phi^{(j)}, \phi^{*}\right)}{q\left(\phi^{*}, \phi^{(j)}\right)}\right)}
$$

This is the general expression for calculating an estimate $\mathrm{p}\left(\phi^{*} \mid \mathbf{d}\right)$ of the posterior 
ordinate, which is then substituted in Eq. (15) to obtain an estimate $\hat{p}(d)$ of the marginal likelihood. Note that we do not perform thinning of MCMC chain generating $\phi^{(g)}$ samples since it has detrimental effect on the statistics of the estimator (See Link and Eaton [70] for detailed treatment of the issue). Next, we explore the performance of the marginal likelihood estimator through a sensitivity study.

\subsubsection{Variability of the marginal likelihood estimate}

As seen from Eq. (23), the choice of $\phi^{*}$, the proposal pdf $q\left(\phi, \phi^{\prime}\right)$ and the sample size $M$ and $J$ will effect the variability and bias of the estimate $\hat{p}(\mathbf{d})$ in repeated MCMC runs. In order to get insight into this effect, we consider a simple numerical case where we compare the analytically obtainable solution of the marginal likelihood with the estimate from the Chib-Jeliazkov method.

Consider a one-dimensional linear model of the form

$$
d=\phi+\epsilon
$$

where $\epsilon \sim \mathcal{N}\left(0, \gamma^{2}\right)$ is a Gaussian white noise, $d$ is the noisy measurement and $\phi$ is an unknown parameter. The prior $\mathrm{p}(\phi)$ of $\phi$ is assumed as $\mathcal{N}\left(\phi \mid \mu, \sigma^{2}\right)$. In that case, the posterior $\mathrm{p}(\phi \mid d)$ will have a $\operatorname{pdf} \mathcal{N}\left(\phi \mid \mu_{f}, \sigma_{f}^{2}\right)$, whose mean and variance can be obtained using one-step Kalman update equations [71] as

$$
\mu_{f}=\frac{\mu \gamma^{2}+d \sigma^{2}}{\sigma^{2}+\gamma^{2}}, \text { and } \sigma_{f}^{2}=\frac{\gamma^{2} \sigma^{2}}{\sigma^{2}+\gamma^{2}}
$$

Once the posterior distribution is known, the exact solution of the marginal likelihood 
can be obtained using the Bayes theorem as

$$
\mathrm{p}(d)=\frac{\mathcal{N}\left(d \mid \phi, \gamma^{2}\right) \mathcal{N}\left(\phi \mid \mu, \sigma^{2}\right)}{\mathcal{N}\left(\phi \mid \mu_{f}, \sigma_{f}^{2}\right)}
$$

Since $p(d)$ does not depends on the value of $\phi$, any value of $\phi$ will give the same $p(d)$ for a given $d$.

In order to obtain the marginal likelihood estimate $\hat{p}(d)$ through the ChibJeliazkov method, we implement a MCMC method based on the Random Walk Metropolis (RWM) algorithm [72-74] to obtain samples from the posterior $\mathrm{p}(\phi \mid d)$ $=\mathcal{N}\left(\phi \mid \mu_{f}, \sigma_{f}^{2}\right)$. We choose the proposal density $q\left(\phi, \phi^{\prime}\right)=\mathcal{N}\left(\phi^{\prime} \mid \phi, \sigma_{p}^{2}\right)$ to generate samples $\phi^{(g)}$. These samples are obtained by discarding some initial stretch of the chain in order to eradicate the effect of the starting point of the chain.

Using Eq. (23), the posterior ordinate estimate $\hat{\mathrm{p}}\left(\phi^{\star} \mid d\right)$ can be obtained using the posterior samples $\phi^{(g)}$ as

$$
\hat{\mathrm{p}}\left(\phi^{\star} \mid d\right)=\frac{\sum_{g=1}^{M} \alpha\left(\phi^{(g)}, \phi^{\star} \mid d\right) \mathcal{N}\left(\phi^{\star} \mid \phi^{(g)}, \sigma_{p}^{2}\right)}{\sum_{j=1}^{M} \alpha\left(\phi^{\star}, \phi^{(j)} \mid d\right)}
$$

where $\phi^{(j)}$ samples are drawn from $\mathcal{N}\left(\phi^{\star} \mid \phi, \sigma_{p}^{2}\right)$ using Monte Carlo sampling [69]. The estimate $\hat{\mathrm{p}}\left(\phi^{\star} \mid d\right)$ is used to obtain an estimate $\hat{\mathrm{p}}(d)$ of the marginal likelihood as

$$
\hat{\mathrm{p}}(d)=\frac{\mathrm{p}\left(d \mid \phi^{\star}\right) \pi\left(\phi^{\star}\right)}{\hat{\mathrm{p}}\left(\phi^{\star} \mid d\right)}=\frac{\mathcal{N}\left(d \mid \phi^{\star}, \gamma^{2}\right) \mathcal{N}\left(\phi^{\star} \mid \mu, \sigma^{2}\right)}{\hat{\mathrm{p}}\left(\phi^{\star} \mid d\right)}
$$

We perform the sensitivity study into two stages. In the first stage, the sample size $M$ of $\phi^{(g)}$ and $\phi^{(j)}$ is kept constant and the proposal variance $\sigma_{p}^{2}$ is varied with respect to the variance $\sigma_{f}^{2}$ of the posterior distribution. In the next stage, the proposal variance $\sigma_{p}^{2}$ is kept constant while the sample size $M$ is varied. Both the cases are studied in the context of varying $\phi^{\star}$ values. The results shown hereafter 
corresponds to the following values: $d=5.0, \mu=2.0, \sigma=5.0$ and $\gamma=2.0$.

\section{Dependence on $\sigma_{p}^{2}$}

The proposal variance $\sigma_{p}^{2}$ is assumed as $\left(w \sigma_{f}\right)^{2}$ for the sake of comparison with the posterior variance $\sigma_{f}{ }^{2}$. Fig. 3 shows the results for six different values of $w$, viz. $0.01,0.05,0.1,1,10$ and 50 for various $\phi^{\star}$ positions in the posterior space. Following inferences can be made from the results:

- For all values of $w$ greater than 1 , the $\hat{\mathrm{p}}(d)$ values are relatively less biased with much less variance for those $\phi^{\star}$ located in the higher posterior probability regions. This argument is in agreement with the one made by Chib [41]. The variance and the bias of the $\hat{\mathrm{p}}(d)$ values is seen to be larger for those $\phi^{\star}$ located on the outskirts of the posterior distribution.

- For all values of $w$ less than 1 , there is a large bias in $\hat{\mathrm{p}}(d)$ values, irrespective of the position of $\phi^{\star}$ in the posterior space. This bias was found for all $w$ less than 0.5 when $M=2000$. This limit of $w$ was found to decrease for increasing sample size $M$ (results not shown here). Hence, choosing the proposal variance on the higher side of the posterior variance will be more effective in obtaining more accurate $\hat{\mathrm{p}}(d)$ values for a fixed $\mathrm{M}$. Similar argument can be expected to hold true for multi-dimensional posterior distributions.

- There is optimal value of proposal variance or $w$ for which the variance of $\hat{p}(d)$ values is very low for those $\phi^{\star}$ located in the higher posterior probability regions. This dependence of the variability of $\hat{\mathrm{p}}(d)$ on the certain range of values of $w$ is attributed to the increase in the correlation for samples $\phi^{(g)}$ for very high or very low $w$ values $[72-74]$. In order to get further insight into this issue, we calculated the estimated integrated autocorrelation time [75] $\hat{\tau}_{\text {int }}$ using $\phi^{(g)}$ 


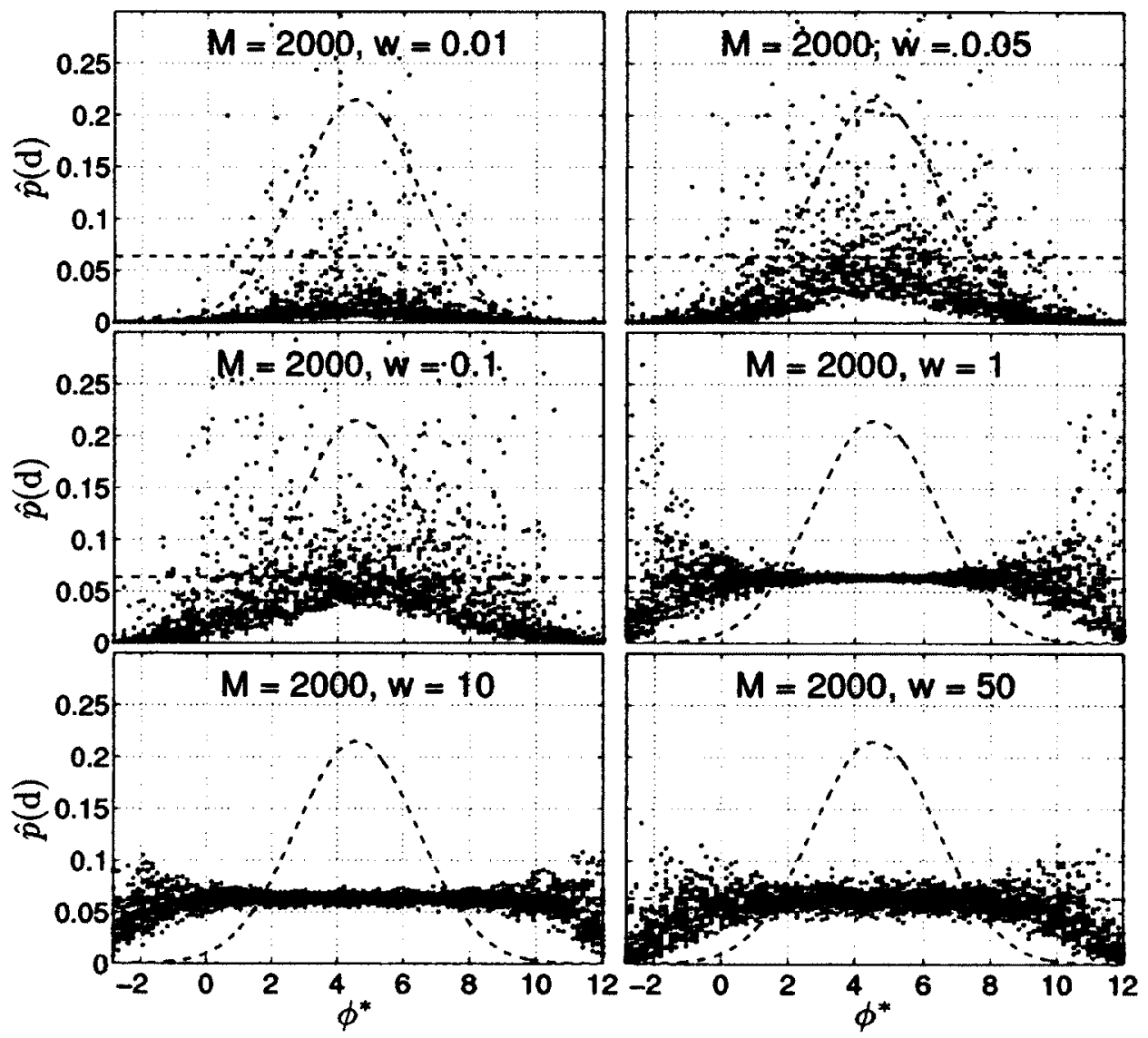

Figure 3: Marginal likelihood estimate for varying $w$ and fixed $M$. 
samples generated for varying $w$ and fixed $\phi^{\star}\left(=\mu_{f}\right)$. We used the XCORR function in Matlab to calculate $\hat{\tau}_{\text {int }}$. The $\hat{\tau}_{\text {int }}$ vs $w$ and $\hat{\mathrm{p}}(d)$ vs $\hat{\tau}_{\text {int }}$ is plotted in Fig. 4 and Fig. 5 respectively.

Fig. 4 and Fig. 5 reinstates the importance of an optimal proposal variance ( $w$ in this case) in obtaining more accurate marginal likelihood estimates. As seen in Fig. 4, the variability of $\hat{\mathrm{p}}(d)$ is less when using least correlated MCMC samples, which is only possible for certain optimal values of $w$. In fact, the value of $w=2.38$ is often proposed in the MCMC literature [72, 73, 76-78] for generating the least correlated (less $\hat{\tau}_{\text {int }}$ values) MCMC samples and best mixing of the Markov chain. As seen in Fig. 4, this argument seems to hold good as the lowest autocorrelation is visible for $w \approx 2.4$. This optimal proposal will provide samples $\phi^{(g)}$ with minimum correlation between subsequent samples and, therefore, a marginal likelihood estimate with less variability. For posterior distribution of dimension $p$, the asymptotic value of $w$ is given by $2.38 / p$ [72]. This value even holds good for the weakly gaussian posterior distribution [72].

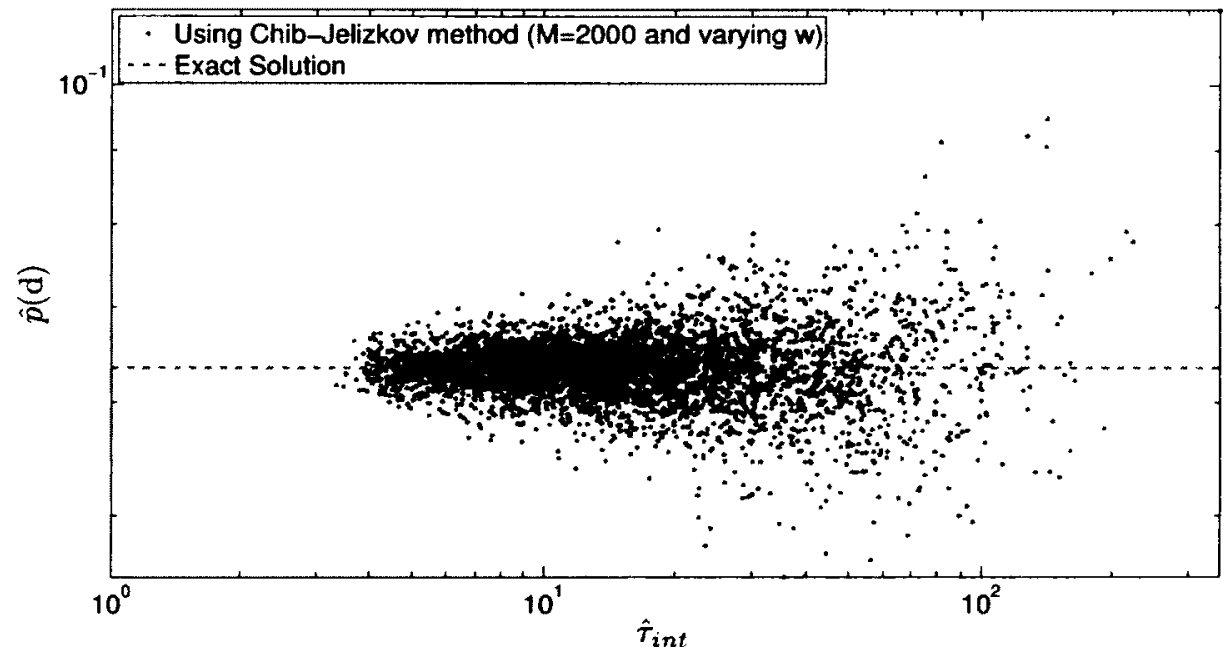

Figure 4: Estimated integrated autocorrelation for varying $w$ and fixed $\phi^{\star}\left(=\mu_{f}\right)$ 


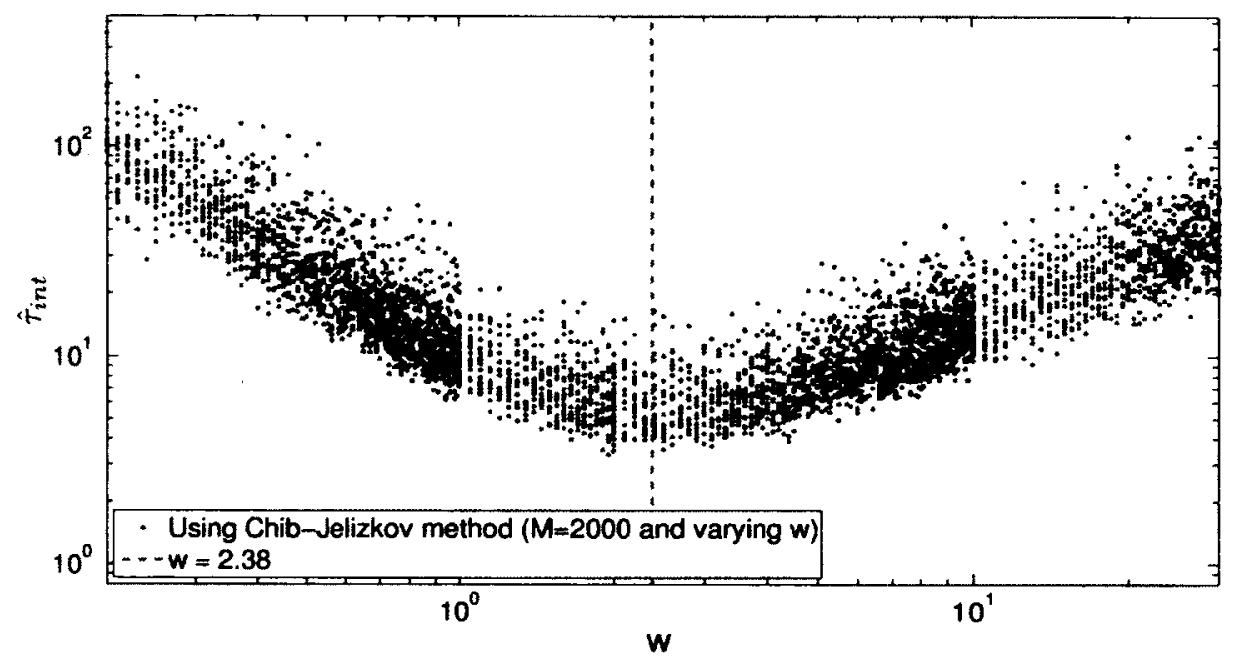

Figure 5: Marginal likelihood estimate for varying estimated integrated autocorrelation time obtained by varying $w$ for fixed $\phi^{\star}\left(=\mu_{f}\right)$

\section{Dependence on $M$}

To study the impact of varying the sample size $M$ on the marginal likelihood estimate $\hat{p}(d)$, the value of $w$ was fixed as 1. The results shown in Fig. 6 corresponds $M$ values of $100,500,1000,2000,5000$ and 10000 . As seen in Fig. 6, the bias and variance of the marginal likelihood estimates is higher for those $\phi^{\star}$ situated on the outskirts of the posterior distribution for any value of $M$. This is similar to what was noticed in the case of varying $w$. Hence, no matter what the values of $w$ and $M$ are, one should always choose $\phi^{\star}$ in high posterior probability regions. 


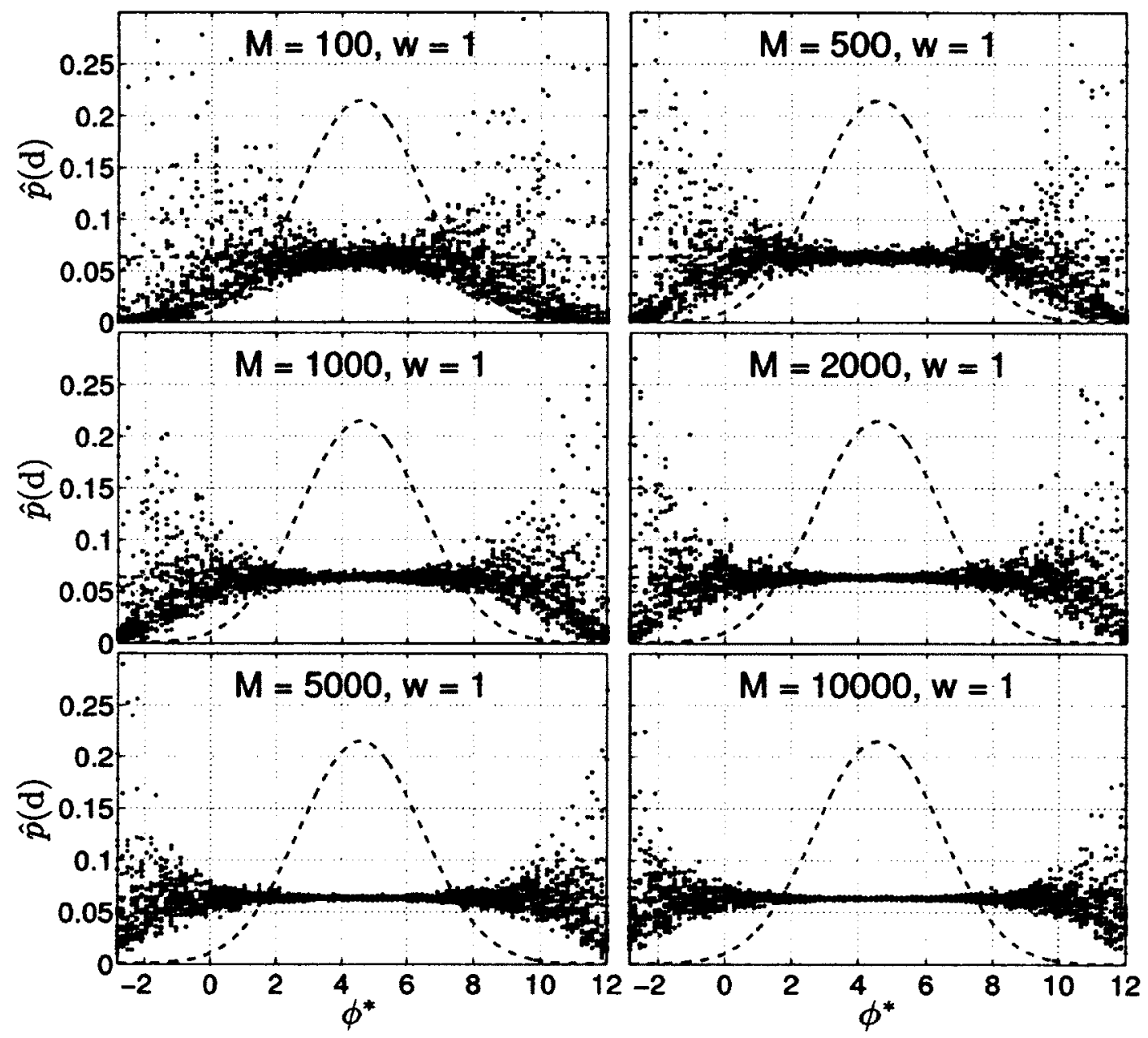

Figure 6: Marginal likelihood estimates for varying $M$ and fixed $w$ 


\subsubsection{Bayes factor sensitivity on prior specifications}

To illustrate the sensitivity of prior pdf on Bayesian model selection, consider the $\Delta \mathrm{BIC}_{i, j}^{\text {exact }}$ equation for the comparison of model $\mathcal{M}_{i}$ and $\mathcal{M}_{j}$,

$$
\Delta \mathrm{BIC}_{i, j}^{\text {exact }}==-2 \log \left\{\frac{\int \mathrm{p}_{i}\left(\mathbf{d} \mid \phi_{i}\right) \mathrm{p}_{i}\left(\phi_{i}\right) d \phi_{i}}{\int \mathrm{p}_{j}\left(\mathbf{d} \mid \phi_{j}\right) \mathrm{p}_{j}\left(\phi_{j}\right) d \phi_{j}}\right\}
$$

The prior pdf $\mathrm{p}_{i}\left(\phi_{i}\right)$ and $\mathrm{p}_{j}\left(\phi_{j}\right)$ represents the knowledge about the model parameters before the data $\mathbf{d}$ is analyzed. Assume that the prior pdf assigned to model $\mathcal{M}_{i}$ is $h_{i}\left(\phi_{i}\right) / c_{i}$ and to model $\mathcal{M}_{j}$ is $h_{j}\left(\phi_{j}\right) / c_{j}$, where $h_{i}\left(\phi_{i}\right)$ and $h_{j}\left(\phi_{j}\right)$ are well-defined pdfs and $c_{i}$ and $c_{j}$ are the constants that control the spread of these pdfs. Assigning these prior pdfs in Eq. (29), we get

$$
\begin{aligned}
\Delta \mathrm{BIC}_{i, j}^{\text {exact }} & =-2 \log \left\{\frac{\int \mathrm{p}_{i}\left(\mathbf{d} \mid \boldsymbol{\phi}_{i}\right)\left\{h_{i}\left(\boldsymbol{\phi}_{i}\right) / c_{i}\right\} d \boldsymbol{\phi}_{i}}{\int \mathrm{p}_{j}\left(\mathbf{d} \mid \boldsymbol{\phi}_{j}\right)\left\{h_{j}\left(\boldsymbol{\phi}_{j}\right) / c_{j}\right\} d \boldsymbol{\phi}_{j}}\right\} \\
& =-2\left[\log \left\{\frac{c_{j}}{c_{i}}\right\}+\log \left\{\frac{\int \mathrm{p}_{i}\left(\mathbf{d} \mid \boldsymbol{\phi}_{i}\right) h_{i}\left(\boldsymbol{\phi}_{i}\right) d \boldsymbol{\phi}_{i}}{\int \mathrm{p}_{j}\left(\mathbf{d} \mid \boldsymbol{\phi}_{j}\right) h_{j}\left(\boldsymbol{\phi}_{j}\right) d \boldsymbol{\phi}_{j}}\right\}\right] .
\end{aligned}
$$

Here, the ratio $c_{j} / c_{i}$ represents the relative spread of the prior pdfs between the two models. A different value of $c_{i}$ and $c_{j}$, i.e. different amount of prior spread for the parameters of the two models will result in a different value of $\Delta \mathrm{BIC}_{i, j}^{\text {exact }}$. This variation of $\Delta \mathrm{BIC}_{i, j}^{\text {exact }}$ will produce inconsistent results when performing model comparison.

To get further insight, consider a case of independent, uniform prior distribution $\left(h_{i}\left(\phi_{i}\right)=h_{j}\left(\phi_{j}\right)=1\right)$ with equal value of constant $c$ for both the models. Consider that the size of $\phi_{i}$ is $p_{i}$ and of $\phi_{j}$ is $p_{j}$. In that case, the prior pdfs are

$$
\mathrm{p}_{i}\left(\phi_{i}\right)=\frac{1}{c} \times \frac{1}{c} \times \ldots p_{i} \text { times } \ldots \times \frac{1}{c}
$$




$$
\mathrm{p}_{j}\left(\phi_{j}\right)=\frac{1}{c} \times \frac{1}{c} \times \ldots p_{j} \text { times } \ldots \times \frac{1}{c}
$$

Assigning these prior pdfs in Eq. (30b), we get

$$
\Delta \mathrm{BIC}_{i, j}^{\text {exact }}=-2\left[\left(p_{j}-p_{i}\right) \log c+\log \left\{\frac{\int \mathrm{p}_{i}\left(\mathbf{d} \mid \phi_{i}\right) d \phi_{i}}{\int \mathrm{p}_{j}\left(\mathbf{d} \mid \phi_{j}\right) d \phi_{j}}\right\}\right]
$$

Hence, for $p_{i} \neq p_{j}$ the $\Delta \mathrm{BIC}_{i, j}^{\text {exact }}$ value is biased by a constant that increases linearly with the logarithm of the spread $c$ of the prior distribution. Hence, for the higher spread $c$, the $\Delta \mathrm{BIC}_{i, j}^{\text {exact }}$ value will be dictated by the difference in the size of model parameter vector of the competing models. For example, if $p_{j}>p_{i}$ for large $c$, there are high chances that $\Delta \mathrm{BIC}_{i, j}^{\text {exact }}$ value will favor the model $M_{i}$ based on the benchmark values in Table 1. Similar argument can be made for $p_{j}<p_{i}$ case.

This behaviour of model selection using $\Delta \mathrm{BIC}_{i, j}^{\text {exact }}$ can be generalized as follows: " $\triangle \mathrm{BIC}_{i, j}^{\text {exact }}$ will always tend to favor the simpler model as the better model, when using relatively less informative priors (large $c$ ) for the competing models". This behaviour is called the Bartletts' paradox [26, 27]. Bartletts' paradox also implies that even though the prior distribution contains very little information about the model parameters, the Bayes factor is highly affected by it and will not produce consistent model comparison results with the variation of $c$ value. The only exception to Bartletts' paradox is when $p_{i}=p_{j}$, i.e. the competing models have same number of parameters.

To avoid the difficulties with assigning informative priors in Bayesian analysis, non-informative or improper priors, representing no or vague knowledge, are often used. A prior pdf $\pi(\phi)$ is improper when $\int_{\phi} p(\phi) d \phi$ diverges. A non-informative prior will have no finite bounds and, therefore, will be an improper prior.

Although non-informative priors result in a well-defined posterior distribution, $\triangle \mathrm{BIC}_{i, j}^{\text {exact }}$ is not well-defined [44]. Hence, the dimension of the competing models 
seems to play an important part in deciding the effect of relative prior specifications on the Bayes factor. As the Bayesian literature developed, a new class of improper priors are being proposed which results in a well-defined Bayes factor. Strachen and Dijk $[26,27]$ has provided an excellent review of various improper priors in this class. Most of these improper priors are designed in such a way that the term $\left(p_{j}-p_{i}\right) \log c$ in Eq. (33) is finite, resulting in a well-defined Bayes factor.

For the stepwise model selection scheme employed in this thesis, at any model selection step the candidate model set is given as $\left\{\left\{\mathcal{M}_{1}, \mathcal{M}_{2}, \ldots, \mathcal{M}_{r}, \ldots, \mathcal{M}_{N}\right\}\right.$, $\left.\left\{\mathcal{M}_{G}\right\}\right\}$, where $\mathcal{M}_{r}$ 's are the reduced nested models with one term less than the global model $\mathcal{M}_{G}$. In order to execute the model selection task, we employ Eq. (33) to compare $\mathcal{M}_{G}$ with any $\mathcal{M}_{r}$ by calculating $\Delta \mathrm{BIC}_{r, G}^{\text {exact }}$ as

$$
\Delta \mathrm{BIC}_{r, G}^{\text {exact }}=-2\left[\log c+\log \left\{\frac{\int \mathrm{p}_{r}\left(\mathbf{d} \mid \boldsymbol{\phi}_{r}\right) d \boldsymbol{\phi}_{r}}{\int \mathrm{p}_{G}\left(\mathbf{d} \mid \boldsymbol{\phi}_{G}\right) d \boldsymbol{\phi}_{G}}\right\}\right] .
$$

In this thesis, we will chose $c=\sqrt{\pi}$ as advocated by Strachen [27]. He arrived at this value by diverging the prior spread to infinity in a spherical manner at such a rate that the value of $c$ is finite. However, as noticeable in Eq. (34), the presence of the term ' $\log c$ ' in calculating $\triangle \mathrm{BIC}_{r, G}^{\text {exact }}$ will not effect the model selection but will only

alter Table 1 by a constant factor. Therefore, we will revisit the $\triangle B I_{r, G}^{\text {exact }}$ benchmark values for the chosen value of ' $c$ ' $(\sqrt{\pi}$ in this case) in Chapter 5 through a numerical study performed in the context of the wing oscillations under consideration.

\subsection{Information-theoretic approach}

Information-theoretic method of model selection is a frequentist approach of comparing the competing models $\mathcal{M}_{i}$, whose parameters $\phi_{i}$ are available in the form of point estimates, denoted as $\hat{\boldsymbol{\phi}}_{i}$. In this thesis, we employ the MLE method $[1,2]$ to 
obtain $\hat{\phi}_{i}$ by maximizing the likelihood function $\mathrm{p}_{i}\left(\mathbf{d} \mid \phi_{i}\right)$. Since we are using noninformative priors for $\phi_{i}$, the mode of the joint posterior $\mathrm{p}_{i}\left(\phi_{i} \mid \mathbf{d}\right)$ is same as $\hat{\phi}_{i}$. Once $\hat{\phi}_{i}$ is available for all $\mathcal{M}_{i}$, information-theoretic methods assign a score to each model, which is then compared over all models in the candidate model set.

A general expression for any information-theoretic criterion is given as

$$
\mathrm{IC}_{i}=-2 \sum_{k=1}^{n} \log \mathrm{p}_{i}\left(d_{k} \mid \hat{\phi}_{i}\right)+[\text { Penalty term }]_{i}
$$

The first term in the above expression represents the goodness of fit while the second term is a penalty factor for the complexity of the model $\mathcal{M}_{i}$. The penalty factor works against the goodness of fit term. Therefore, the model with a minimum value of $\mathrm{IC}_{i}$ will represent an optimality between the goodness of fit and the complexity of the model over all competing models.

In this thesis, we consider three information criteria, namely $\mathrm{AIC}, \mathrm{BIC}$ and ICOMP for contrasting the performance of the $\mathrm{BIC}^{\text {exact }}$ criterion. AIC is obtained by approximating the $\mathrm{K}-\mathrm{L}$ information assuming that the model being compared exists near the true model - a hypothetical model representing the true physics of the system [1]. Since the true model does not exists in practice, the assumption is irrelevant from the practical point of view. BIC is obtained by approximating the integral involved in $\mathrm{BIC}^{\text {exact }}$ through Laplace method for a large data d [1]. However, there is no formal definition for what defines 'large data' [44]. ICOMP is a recently proposed frequentist method that penalizes the complex models based on the correlation structure of their model parameters [48].

The final expressions for AIC, BIC and ICOMP for each model $\mathcal{M}_{i}$ is given as

$$
\mathrm{AIC}_{i}=-2 \sum_{k=1}^{n} \log \mathrm{p}_{i}\left(d_{k} \mid \hat{\boldsymbol{\phi}}_{i}\right)+2 p_{i}
$$




$$
\mathrm{BIC}_{i}=-2 \sum_{k=1}^{n} \log \mathrm{p}_{i}\left(d_{k} \mid \hat{\phi}_{i}\right)+p_{i} \log n
$$

and

$$
\operatorname{ICOMP}_{i}=-2 \sum_{k=1}^{n} \log \mathrm{p}_{i}\left(d_{k} \mid \hat{\phi}_{i}\right)+2 p_{i} \log \frac{\operatorname{tr}\left(\hat{\Sigma}_{i}\right)}{p_{i}}-\log \left|\hat{\Sigma}_{i}\right|
$$

Here, $\hat{\Sigma}_{i}$ is an estimate of the covariance matrix $\Sigma_{i}$ of the model parameters $\phi_{i}$, which is readily available as a by-product from the one-block M-H sampling algorithm employed in this thesis.

In the context of the stepwise model selection scheme, we calculate $\Delta \mathrm{IC}_{r, G}$ for the three criterion to result in $\triangle \mathrm{AIC}_{r, G}, \Delta \mathrm{BIC}_{r, G}$ and $\triangle \mathrm{ICOMP}_{r, G}$ as follows

$$
\begin{aligned}
\Delta \mathrm{IC}_{r, G} & =-2\left[\sum_{k=1}^{n} \log \mathrm{p}_{r}\left(d_{k} \mid \hat{\boldsymbol{\phi}}_{r}\right)+[\text { Penalty term }]_{r}-\sum_{k=1}^{n} \log \mathrm{p}_{G}\left(d_{k} \mid \hat{\boldsymbol{\phi}}_{G}\right)+[\text { Penalty term }]_{G}\right] \\
& =-2\left[\sum_{k=1}^{n} \log \left\{\frac{\mathrm{p}_{r}\left(d_{k} \mid \hat{\boldsymbol{\phi}}_{r}\right)}{\mathrm{p}_{G}\left(d_{k} \mid \hat{\phi}_{G}\right)}\right\}+[\text { Penalty term }]_{r}-[\text { Penalty term }]_{G}\right]
\end{aligned}
$$




\section{Chapter 4}

\section{Methodology: Parameter estimation}

In this chapter, we present a Bayesian inference based parameter estimation methodology. First, we define the state space model structure for which the methodology is applicable. Then, using Bayesian inference, we pose a combined state and parameter estimation problem, wherein the state estimation is performed using the EKF. Finally, we discuss the adaptive MCMC sampling technique used for sampling from the posterior distribution.

\subsection{State Space Models}

In this thesis, we deal with the dynamical systems whose physics is modeled by a nonlinear stochastic ordinary differential equation. Once a noisy measurement data d is acquired from the system, the overall model $\mathcal{M}$ is represented in a discrete state-space structure as $[57,58,79]$

$$
\begin{array}{lr}
\mathbf{x}_{k+1}=\mathbf{g}_{k}\left(\mathbf{x}_{k}, \mathbf{f}_{k}, \mathbf{q}_{k}\right) & \text { - Dynamic Model } \\
\mathbf{d}_{k}=\mathbf{h}_{k}\left(\mathbf{x}_{k}, \boldsymbol{\epsilon}_{k}\right) & \text { - Measurement Model. }
\end{array}
$$


Here $\mathbf{x} \in \mathbb{R}^{n}$ is the state of the system, $\mathbf{g} \in \mathbb{R}^{n}$ is the discrete dynamic operator, f $\in \mathbb{R}^{p}$ is a discrete deterministic input and $\mathbf{h} \in \mathbb{R}^{m}$ is the discrete measurement operator. The uncertainty in the dynamic and measurement models is represented by the modelling error $\mathbf{q} \in \mathbb{R}^{s}$ and the measurement error $\epsilon \in \mathbb{R}^{r}$, respectively. $\mathbf{q}$ and $\boldsymbol{\epsilon}$ are assumed to be independent gaussian vectors with mean $\overline{\mathbf{q}} \in \mathbb{R}^{s}$ and $\overline{\boldsymbol{\epsilon}} \in \mathbb{R}^{r}$ and covariance matrices $\mathbf{Q} \in \mathbb{R}^{s \times s}$ and $\Gamma \in \mathbb{R}^{r \times r}$ respectively.

In Eq. (40), the dynamic operator $\mathbf{g}($.$) capture the stochastic dynamics of the$ system while the measurement operator $\mathbf{h}($.$) relates the measurable quantity acquired$ in the data $\mathbf{d}$ to the state $\mathbf{x}$ of the system. The operator $\mathbf{g}($.$) is also characterized by$ an unknown time-invariant parameter vector $\phi$, not shown explicitly in Eq. (40). The parameter estimation task involves estimating $\phi$ given the model $\mathcal{M}$ and the data $\mathbf{d}$.

The model $\mathcal{M}$ in Eq. (40) can also be viewed as a recursively defined conditional probability model of the form [58]

$$
\begin{array}{rr}
\mathbf{x}_{k+1} \sim \mathrm{p}\left(\mathbf{x}_{k+1} \mid \mathbf{x}_{k}\right) & \text { - Dynamic Model } \\
\mathbf{d}_{k} \sim \mathrm{p}\left(\mathbf{d}_{k} \mid \mathbf{x}_{k}\right) & - \text { Measurement Model. }
\end{array}
$$

In other words, the state $\mathbf{x}_{k+1}$ and data $\mathbf{d}_{k}$ are random variables with conditional distributions $\mathrm{p}\left(\mathbf{x}_{k+1} \mid \mathbf{x}_{k}\right)$ and $\mathrm{p}\left(\mathbf{d}_{k} \mid \mathbf{x}_{k}\right)$ respectively. Throughout this thesis, we will assume the Markovian nature of the conditional distributions in Eq. (41). This means that [58]:

1. The states form a Markov sequence. This implies that the future state $\mathbf{x}_{k+1}$ given the current state $\mathbf{x}_{k}$ is independent of all the other past state and data history, which means $\mathrm{p}\left(\mathbf{x}_{k+1} \mid \mathbf{x}_{1: k}, \mathbf{d}_{1: k}\right)=\mathrm{p}\left(\mathbf{x}_{k+1} \mid \mathbf{x}_{k}\right)$.

2. The data $d_{k}$ given the state $x_{k}$ is independent of all the past state and data history, which means $\mathrm{p}\left(\mathbf{d}_{k} \mid \mathbf{x}_{1: k}, \mathbf{d}_{1: k-1}\right)=\mathrm{p}\left(\mathbf{d}_{k} \mid \mathbf{x}_{k}\right)$. 


\subsection{Bayesian Inference}

We estimate the unknown parameter $\phi$ of the model $\mathcal{M}$ by incorporating the noisy data $\mathbf{d}$ through Bayesian inference [5, 56-58]. In Bayesian settings, any information about $\phi$ that exists prior to analyzing the data $\mathbf{d}$ is represented in the form of a prior pdf $\mathrm{p}(\phi)$. The model structure $\mathcal{M}$ is incorporated through a $\operatorname{pdf} \mathrm{p}(\mathrm{d} \mid \phi)$, often called as the forward pdf or likelihood function. In general, Bayesian inference provides a statistical framework to update the prior $\operatorname{pdf} p(\phi)$ by incorporating the noisy data $d$ through the likelihood function $\mathrm{p}(\mathbf{d} \mid \phi)$ to result in a posterior $\operatorname{pdf} \mathrm{p}(\phi \mid \mathbf{d})$. Here, the posterior $\mathrm{p}(\phi \mid \mathrm{d})$ represents the post-data state of information about $\phi$.

Mathematically speaking, Bayesian inference employs Bayes theorem to obtained the posterior $\mathrm{p}(\phi \mid \mathrm{d})$ as $[5,58]$

$$
\mathrm{p}(\boldsymbol{\phi} \mid \mathbf{d})=\frac{\mathrm{p}(\mathbf{d} \mid \phi) \mathrm{p}(\phi)}{\mathrm{p}(\mathbf{d})}
$$

where $\mathrm{p}(\mathbf{d})=\int \mathrm{p}(\mathbf{d} \mid \phi) \mathrm{p}(\boldsymbol{\phi}) d \boldsymbol{\phi}$ is a normalizing constant, also known as the marginal likelihood or the evidence. As discussed in Chapter 3, the marginal likelihood plays an important role in the Bayesian model selection procedure.

Since we are considering nonlinear and high dimensional (roughly 5-25 sized $\phi)$ models, the integration involved in obtaining $p(\mathbf{d})$ is avoided for the sake of parameter estimation to re-write Eq. (42) as

$$
\mathrm{p}(\boldsymbol{\phi} \mid \mathbf{d}) \propto \mathrm{p}(\mathbf{d} \mid \phi) \mathrm{p}(\phi)
$$

In this thesis, we will sample the posterior $\mathrm{p}(\phi \mid \mathbf{d})$ in Eq. (43) using a M-H based 
MCMC sampling algorithm. MCMC methods do not require the normalizing constant to be known and, hence, are best suited for sampling from distributions like Eq. (43).

Considering that the measurement data vector $\mathbf{d}_{1: n}=\left\{\mathbf{d}_{1}, \mathbf{d}_{2}, \ldots, \mathbf{d}_{n}\right\}$, Eq. (43) can be expanded as $[5,58]$

$$
\begin{aligned}
\mathrm{p}\left(\boldsymbol{\phi} \mid \mathbf{d}_{1: n}\right) & \propto \mathrm{p}\left(\mathbf{d}_{1}, \mathbf{d}_{2}, \ldots, \mathbf{d}_{k}, \ldots, \mathbf{d}_{n} \mid \boldsymbol{\phi}\right) \mathrm{p}(\boldsymbol{\phi}) \\
& \propto \mathrm{p}(\boldsymbol{\phi}) \prod_{k=1}^{n} \mathrm{p}\left(\mathbf{d}_{k} \mid \mathbf{d}_{1: k-1}, \boldsymbol{\phi}\right) .
\end{aligned}
$$

The pdf $\mathrm{p}\left(\mathrm{d}_{k} \mid \mathbf{d}_{1: k-1}, \phi\right)$ is obtained by integrating out the state $\mathbf{x}_{k}$ from the joint pdf $\mathrm{p}\left(\mathbf{d}_{k}, \mathbf{x}_{k} \mid \mathbf{d}_{1: k-1}, \phi\right)$ to rewrite Eq. (44b) as

$$
\begin{aligned}
\mathrm{p}\left(\phi \mid \mathbf{d}_{1: n}\right) & \propto \mathrm{p}(\phi) \prod_{k=1}^{n} \int \mathrm{p}\left(\mathbf{d}_{k}, \mathbf{x}_{k} \mid \mathbf{d}_{1: k-1}, \phi\right) d \mathbf{x}_{k} \\
& \propto \mathrm{p}(\phi) \prod_{k=1}^{n} \int \mathrm{p}\left(\mathbf{d}_{k} \mid \mathbf{x}_{k}, \mathbf{d}_{1: k-1}, \phi\right) \mathrm{p}\left(\mathbf{x}_{k} \mid \mathbf{d}_{1: k-1}, \phi\right) d \mathbf{x}_{k} \\
& \propto \mathrm{p}(\phi) \prod_{k=1}^{n} \int \mathrm{p}\left(\mathbf{d}_{k} \mid \mathbf{x}_{k}, \phi\right) \mathrm{p}\left(\mathbf{x}_{k} \mid \mathbf{d}_{1: k-1}, \phi\right) d \mathbf{x}_{k}
\end{aligned}
$$

The pdf $\mathrm{p}\left(\mathrm{x}_{k} \mid \mathrm{d}_{1: k-1}, \phi\right)$ represents the state distribution at the $\mathrm{k}^{\text {th }}$ time step, given all the previous data history, i.e. $\mathbf{d}_{1: k-1}$. We obtain this pdf by performing state estimation using the EKF. Due to the state estimation step involved in obtaining the posterior $\mathrm{p}\left(\phi \mid \mathrm{d}_{1: n}\right)$, the following procedure is also called the combined state and parameter estimation. 


\subsection{State Estimation Using the Extended Kalman Filter}

The KF is a mathematical tool that provides an optimal solution for the posterior state distribution conditional upon the measurement data for linear measurement and dynamic model operators $[71,80,81]$. The state distribution is propagated as a gaussian distribution whose analytical solution is obtained through a recursive assimilation of the data. The assumption of gaussian state distribution is attributed to its analytical tractability under linear mathematical operations. For nonlinear model structures, the Kalman filtering process is augmented by a linearizing procedure, leading to the EKF $[80,81]$.

In EKF, the nonlinear operators are linearized by a first-order Taylor series expansion [80]. Although EKF is not optimal in strict sense, the state estimation is successfully performed due to the availability of the dense data set $\mathbf{d}[80]$. Next, assuming that the model structure resembles the one in Eq. (40), the EKF procedure is described through recursive analysis and forecast steps.

\section{Analysis step $[80,81]$}

We consider that the pdf $\mathrm{p}\left(\mathbf{x}_{k} \mid \mathbf{d}_{1: k-1}, \phi\right)$ is available as $\mathcal{N}\left(\mathbf{x}_{k} \mid \mathbf{x}_{k}^{f}, \mathbf{P}_{k}^{f}\right)$ from the forecast step of $(\mathrm{k}-1)^{t h}$ time step. The measurement operator $\mathbf{h}_{k}\left(\mathbf{x}_{k}, \boldsymbol{\epsilon}_{k}\right)$ is linearized at the mean forecast $\mathbf{x}_{k}^{f}$ using the first order Taylor series expansion as [80, 81]

$$
\mathbf{h}_{k}\left(\mathbf{x}_{k}, \boldsymbol{\epsilon}_{k}\right) \approx \mathbf{h}_{k}\left(\mathbf{x}_{k}^{f}, \overline{\boldsymbol{\epsilon}}_{k}\right)+\mathbf{C}_{k}\left(\mathbf{x}_{k}-\mathbf{x}_{k}^{f}\right)+\mathbf{D}_{k}\left(\boldsymbol{\epsilon}_{k}-\overline{\boldsymbol{\epsilon}}_{k}\right)
$$


where $\mathbf{C}_{k}$ and $\mathbf{D}_{k}$ are the Jacobian matrices of $\mathbf{h}_{k}\left(\mathbf{x}_{k}, \boldsymbol{\epsilon}_{k}\right)$ and are obtained as

$$
\begin{aligned}
& \mathbf{C}_{k}=\left.\frac{\partial \mathbf{h}_{k}\left(\mathbf{x}_{k}, \boldsymbol{\epsilon}_{k}\right)}{\partial \mathbf{x}_{k}}\right|_{\mathbf{x}_{k}=\mathbf{x}_{k}^{f}, \boldsymbol{\epsilon}_{k}=\bar{\epsilon}_{k}} \\
& \mathbf{D}_{k}=\left.\frac{\partial \mathbf{h}_{k}\left(\mathbf{x}_{k}, \boldsymbol{\epsilon}_{k}\right)}{\partial \boldsymbol{\epsilon}_{k}}\right|_{\mathbf{x}_{k}=\mathbf{x}_{k}^{f}, \boldsymbol{\epsilon}_{k}=\bar{\epsilon}_{k}}
\end{aligned}
$$

Substituting Eq. (46) in the measurement model in Eq. (40), we get

$$
\mathbf{d}_{k}=\mathbf{h}_{k}\left(\mathbf{x}_{k}^{f}, \bar{\epsilon}_{k}\right)+\mathbf{C}_{k}\left(\mathbf{x}_{k}-\mathbf{x}_{k}^{f}\right)+\mathbf{D}_{k}\left(\boldsymbol{\epsilon}_{k}-\overline{\boldsymbol{\epsilon}}_{k}\right)
$$

Since $\mathrm{p}\left(\mathbf{x}_{k} \mid \mathbf{d}_{1: k-1}, \phi\right)=\mathcal{N}\left(\mathbf{x}_{k} \mid \mathbf{x}_{k}^{f}, \mathbf{P}_{k}^{f}\right)$, the joint distribution $\mathrm{p}\left(\mathbf{x}_{k}, \mathbf{d}_{k} \mid \mathbf{d}_{1: k-1}\right)$ will be gaussian and can be written as

$$
\mathrm{p}\left(\mathbf{x}_{k}, \mathbf{d}_{k} \mid \mathbf{d}_{1: k-1}, \boldsymbol{\phi}\right)=\mathcal{N}\left(\left[\begin{array}{c}
\mathbf{x}_{k} \\
\mathbf{d}_{k}
\end{array}\right],\left(\begin{array}{c}
\mathbf{x}_{k}^{f} \\
\mathrm{E}\left[\mathbf{d}_{k}\right]
\end{array}\right), \mathbf{P}^{\prime}\right)
$$

where

$$
\mathbf{P}^{\prime}=\left(\begin{array}{cc}
\mathbf{P}_{k}^{f} & \mathrm{E}\left[\left(\mathbf{x}_{k}-\mathbf{x}_{k}^{f}\right)\left(\mathbf{d}_{k}-\mathrm{E}\left[\mathbf{d}_{k}\right]\right)^{T}\right] \\
\mathrm{E}\left[\left(\mathbf{d}_{k}-\mathrm{E}\left[\mathbf{d}_{k}\right]\right)\left(\mathbf{x}_{k}-\mathbf{x}_{k}^{f}\right)^{T}\right] & \mathrm{E}\left[\left(\mathbf{d}_{k}-\mathrm{E}\left[\mathbf{d}_{k}\right]\right)\left(\mathbf{d}_{k}-\mathrm{E}\left[\mathbf{d}_{k}\right]\right)^{T}\right]
\end{array}\right)
$$

We can find $E\left[\mathbf{d}_{k}\right]$ by taking expectation on both sides of Eq. (48) as

$$
\mathrm{E}\left[\mathbf{d}_{k}\right]=\mathrm{E}\left[\mathbf{h}_{k}\left(\mathbf{x}_{k}^{f}, \overline{\boldsymbol{\epsilon}}_{k}\right)\right]+\mathrm{E}\left[\mathbf{C}_{k}\left(\mathbf{x}_{k}-\mathbf{x}_{k}^{f}\right)\right]+\mathrm{E}\left[\mathbf{D}_{k}\left(\boldsymbol{\epsilon}_{k}-\overline{\boldsymbol{\epsilon}}_{k}\right)\right]
$$


Since $\mathbf{x}_{k}$ and $\epsilon_{k}$ are the only random vectors on the right-hand side and $E\left[\mathbf{x}_{k}\right]=\mathbf{x}_{k}^{f}$ and $\mathrm{E}\left[\boldsymbol{\epsilon}_{k}\right]=\bar{\epsilon}_{k}$, Eq. (51) is reduced to

$$
\mathrm{E}\left[\mathbf{d}_{k}\right]=\mathbf{h}_{k}\left(\mathbf{x}_{k}^{f}, \overline{\boldsymbol{\epsilon}}_{k}\right)
$$

Assuming $\mathrm{E}\left[\left(\mathbf{x}_{k}-\mathbf{x}_{k}^{f}\right)\left(\boldsymbol{\epsilon}_{k}-\overline{\boldsymbol{\epsilon}}_{k}\right)^{T}\right]=0$, the variance $\mathrm{E}\left[\left(\mathbf{d}_{k}-\mathrm{E}\left[\mathbf{d}_{k}\right]\right)\left(\mathbf{d}_{k}-\mathrm{E}\left[\mathbf{d}_{k}\right]\right)^{T}\right]$ can be obtained using Eq. (48) and Eq. (52) as

$$
\begin{aligned}
& \mathrm{E}\left[\left(\mathbf{d}_{k}-\mathrm{E}\left[\mathbf{d}_{k}\right]\right)\left(\mathbf{d}_{k}-\mathrm{E}\left[\mathbf{d}_{k}\right]\right)^{T}\right] \\
& \quad=\mathrm{E}\left[\left(\mathbf{d}_{k}-\mathbf{h}_{k}\left(\mathbf{x}_{k}^{f}, \bar{\epsilon}_{k}\right)\right)\left(\mathbf{d}_{k}-\mathbf{h}_{k}\left(\mathbf{x}_{k}^{f}, \overline{\boldsymbol{\epsilon}}_{k}\right)\right)^{T}\right] \\
& \quad=\mathrm{E}\left[\left(\mathbf{C}_{k}\left(\mathbf{x}_{k}-\mathbf{x}_{k}^{f}\right)+\mathbf{D}_{k}\left(\boldsymbol{\epsilon}_{k}-\bar{\epsilon}_{k}\right)\right)\left(\mathbf{C}_{k}\left(\mathbf{x}_{k}-\mathbf{x}_{k}^{f}\right)+\mathbf{D}_{k}\left(\boldsymbol{\epsilon}_{k}-\bar{\epsilon}_{k}\right)\right)^{T}\right] \\
& \quad=\mathrm{E}\left[\left(\mathbf{C}_{k}\left(\mathbf{x}_{k}-\mathbf{x}_{k}^{f}\right)+\mathbf{D}_{k}\left(\boldsymbol{\epsilon}_{k}-\bar{\epsilon}_{k}\right)\right)\left(\left(\mathbf{x}_{k}-\mathbf{x}_{k}^{f}\right)^{T} \mathbf{C}_{k}^{T}+\left(\boldsymbol{\epsilon}_{k}-\bar{\epsilon}_{k}\right)^{T} \mathbf{D}_{k}^{T}\right)\right] \\
& \quad=\mathbf{C}_{k} \mathrm{E}\left[\left(\mathbf{x}_{k}-\mathbf{x}_{k}^{f}\right)\left(\mathbf{x}_{k}-\mathbf{x}_{k}^{f}\right)^{T}\right] \mathbf{C}_{k}^{T}+\mathbf{D}_{k} \mathrm{E}\left[\left(\boldsymbol{\epsilon}_{k}-\bar{\epsilon}_{k}\right)\left(\boldsymbol{\epsilon}_{k}-\bar{\epsilon}_{k}\right)^{T}\right] \mathbf{D}_{k}^{T} \\
& \quad=\mathbf{C}_{k} \mathbf{P}_{k}^{f} \mathbf{C}_{k}^{T}+\mathbf{D}_{k} \boldsymbol{\Gamma}_{k} \mathbf{D}_{k}^{T}
\end{aligned}
$$

Using Eq. (53f) and Eq. (52), we can write

$$
\mathrm{p}\left(\mathbf{d}_{k} \mid \mathbf{d}_{1: k-1}, \phi\right)=\mathrm{p}\left(\mathbf{d}_{k} \mid \phi\right)=\mathcal{N}\left(\mathbf{d}_{k} \mid \mathbf{h}_{k}\left(\mathbf{x}_{k}^{f}, \bar{\epsilon}_{k}\right), \mathbf{C}_{k} \mathbf{P}_{k}^{f} \mathbf{C}_{k}^{T}+\mathbf{D}_{k} \boldsymbol{\Gamma}_{k} \mathbf{D}_{k}^{T}\right)
$$

The cross-variance $\mathrm{E}\left[\left(\mathbf{d}_{k}-\mathrm{E}\left[\mathbf{d}_{k}\right]\right)\left(\mathbf{x}_{k}-\mathbf{x}_{k}^{f}\right)^{T}\right]$ is calculated as

$$
\begin{aligned}
& \mathrm{E}\left[\left(\mathbf{d}_{k}-\mathrm{E}\left[\mathbf{d}_{k}\right]\right)\left(\mathbf{x}_{k}-\mathbf{x}_{k}^{f}\right)^{T}\right] \\
& \quad=\mathrm{E}\left[\left(\mathbf{d}_{k}-\mathbf{h}_{k}\left(\mathbf{x}_{k}^{f}, \bar{\epsilon}_{k}\right)\right)\left(\mathbf{x}_{k}-\mathbf{x}_{k}^{f}\right)^{T}\right] \\
& \quad=\mathrm{E}\left[\left(\mathbf{C}_{k}\left(\mathbf{x}_{k}-\mathbf{x}_{k}^{f}\right)+\mathbf{D}_{k}\left(\epsilon_{k}-\bar{\epsilon}_{k}\right)\right)\left(\mathbf{x}_{k}-\mathbf{x}_{k}^{f}\right)^{T}\right]
\end{aligned}
$$




$$
\begin{aligned}
& =\mathbf{C}_{k} \mathrm{E}\left[\left(\mathbf{x}_{k}-\mathbf{x}_{k}^{f}\right)\left(\mathbf{x}_{k}-\mathbf{x}_{k}^{f}\right)^{T}\right] \\
& =\mathbf{C}_{k} \mathbf{P}_{k}^{f}
\end{aligned}
$$

Hence, using Eq. (51), Eq. (53f) and Eq. (143d), we can write

$$
\mathrm{p}\left(\mathbf{x}_{k}, \mathbf{d}_{k} \mid \mathbf{d}_{1: k-1}, \boldsymbol{\phi}\right)=\mathcal{N}\left(\left[\begin{array}{l}
\mathbf{x}_{k} \\
\mathbf{d}_{k}
\end{array}\right] \mid\left(\begin{array}{c}
\mathbf{x}_{k}^{f} \\
\mathbf{h}_{k}\left(\mathbf{x}_{k}^{f}, \overline{\boldsymbol{\epsilon}}_{k}\right)
\end{array}\right),\left(\begin{array}{cc}
\mathbf{P}_{k}^{f} & \mathbf{P}_{k}^{f} \mathbf{C}_{k}^{T} \\
\mathbf{C}_{k} \mathbf{P}_{k}^{f} & \mathbf{C}_{k} \mathbf{P}_{k}^{f} \mathbf{C}_{k}^{T}+\mathbf{D}_{k} \boldsymbol{\Gamma}_{k} \mathbf{D}_{k}^{T}
\end{array}\right)\right)
$$

Using Eq. (56), we can obtain the updated state pdf $\mathrm{p}\left(\mathbf{x}_{k} \mid \mathbf{d}_{k}, \mathbf{d}_{1: k-1}, \phi\right)$ or $\mathrm{p}\left(\mathbf{x}_{k} \mid \mathbf{d}_{1: k}, \boldsymbol{\phi}\right)$ using gaussian identities shown in Appendix $\mathrm{C}$ as

$$
\mathrm{p}\left(\mathbf{x}_{k} \mid \mathbf{d}_{1: k}, \phi\right)=\mathcal{N}\left(\mathbf{x}_{k} \mid \mathbf{x}_{k}^{a}, \mathbf{P}_{k}^{a}\right)
$$

where

$$
\begin{aligned}
\mathbf{x}_{k}^{a} & =\mathbf{x}_{k}^{f}+\mathbf{K}_{k}\left(\mathbf{d}_{k}-\mathbf{h}_{k}\left(\mathbf{x}_{k}^{f}, \bar{\epsilon}_{k}\right)\right) \\
\mathbf{P}_{k}^{a} & =\left(\mathbf{I}-\mathbf{K}_{k} \mathbf{C}_{k}\right) \mathbf{P}_{k}^{f} \\
\mathbf{K}_{k} & =\mathbf{P}_{k}^{f} \mathbf{C}_{k}^{T}\left(\mathbf{C}_{k} \mathbf{P}_{k}^{f} \mathbf{C}_{k}^{T}+\mathbf{D}_{k} \boldsymbol{\Gamma}_{k} \mathbf{D}_{k}^{T}\right)^{-1} .
\end{aligned}
$$

Hence, in the analysis step, EKF updates forecasted state pdf $\mathrm{p}\left(\mathbf{x}_{k} \mid \mathbf{d}_{1: k-1}, \phi\right)=$ $\mathcal{N}\left(\mathbf{x}_{k} \mid \mathbf{x}_{k}^{f}, \mathbf{P}_{k}^{f}\right)$ by incorporating $\mathbf{d}_{k}$ using Eq. (58) leading to the post-data state pdf $\mathrm{p}\left(\mathbf{x}_{k} \mid \mathbf{d}_{1: k}, \phi\right)=\mathcal{N}\left(\mathbf{x}_{k} \mid \mathbf{x}_{k}^{a}, \mathbf{P}_{k}^{a}\right)$. Next, we discuss the forecast step.

Forecast step $[80,81]$ 
Consider that the current (updated) state $\operatorname{pdf} \mathrm{p}\left(\mathbf{x}_{k} \mid \mathbf{d}_{1: k}, \phi\right)=\mathcal{N}\left(\mathbf{x}_{k} \mid \mathbf{x}_{k}^{a}, \mathbf{P}_{k}^{a}\right)$ is available at the $\mathrm{k}^{\text {th }}$ time step from the analysis step of the EKF. The dynamic model operator is linearized at the state mean $\mathbf{x}_{k}^{a}$ using the first order Taylor series expansion as $[80,81]$

$$
\mathbf{g}_{k}\left(\mathbf{x}_{k}, \mathbf{f}_{k}, \mathbf{q}_{k}\right) \approx \mathbf{g}_{k}\left(\mathbf{x}_{k}^{a}, \mathbf{f}_{k}, \overline{\mathbf{q}}_{k}\right)+\mathbf{A}_{k}\left(\mathbf{x}_{k}-\mathbf{x}_{k}^{a}\right)+\mathbf{B}_{k}\left(\mathbf{q}_{k}-\overline{\mathbf{q}}_{k}\right)
$$

where $\mathbf{A}_{k}$ and $\mathbf{B}_{k}$ are the Jacobian matrices of $\mathbf{g}_{k}\left(\mathbf{x}_{k}, \mathbf{f}_{k}, \mathbf{q}_{k}\right)$ and are obtained as

$$
\begin{aligned}
& \mathbf{A}_{k}=\left.\frac{\partial \mathbf{g}_{k}\left(\mathbf{x}_{k}, \mathbf{f}_{k}, \mathbf{q}_{k}\right)}{\partial \mathbf{x}_{k}}\right|_{\mathbf{x}_{k}=\mathbf{x}_{k}^{a}, \mathbf{q}_{k}=\overline{\mathbf{q}}_{k}} \\
& \mathbf{B}_{k}=\left.\frac{\partial \mathbf{g}_{k}\left(\mathbf{x}_{k}, \mathbf{f}_{k}, \mathbf{q}_{k}\right)}{\partial \mathbf{q}_{k}}\right|_{\mathbf{x}_{k}=\mathbf{x}_{k}^{a}, \mathbf{q}_{k}=\overline{\mathbf{q}}_{k} .}
\end{aligned}
$$

Substituting Eq. (59) in the dynamic model in Eq. (40), we get

$$
\mathbf{x}_{k+1} \approx \mathbf{g}_{k}\left(\mathbf{x}_{k}^{a}, \mathbf{f}_{k}, \overline{\mathbf{q}}_{k}\right)+\mathbf{A}_{k}\left(\mathbf{x}_{k}-\mathbf{x}_{k}^{a}\right)+\mathbf{B}_{k}\left(\mathbf{q}_{k}-\overline{\mathbf{q}}_{k}\right)
$$

The current state pdf $\mathrm{p}\left(\mathbf{x}_{k} \mid \mathbf{d}_{1: k}, \boldsymbol{\phi}\right)=\mathcal{N}\left(\mathbf{x}_{k} \mid \mathbf{x}_{k}^{a}, \mathbf{P}_{k}^{a}\right)$ is then projected to $(\mathrm{k}+1)^{\text {th }}$ time step using Eq. (61) leading to the forecasted state pdf $\mathrm{p}\left(\mathbf{x}_{k+1} \mid \mathbf{d}_{1: k}, \boldsymbol{\phi}\right)=\mathcal{N}\left(\mathbf{x}_{k+1} \mid \mathbf{x}_{k+1}^{f}, \mathbf{P}_{k+1}^{f}\right)$. The mean state $\mathbf{x}_{k+1}^{f}$ is obtained by taking expectation of Eq. (61) as

$$
\mathbf{x}_{k+1}^{f}=\mathrm{E}\left[\mathbf{g}_{k}\left(\mathbf{x}_{k}^{a}, \mathbf{f}_{k}, \overline{\mathbf{q}}_{k}\right)\right]+\mathrm{E}\left[\mathbf{A}_{k}\left(\mathbf{x}_{k}-\mathbf{x}_{k}^{a}\right)\right]+\mathrm{E}\left[\mathbf{B}_{k}\left(\mathbf{q}_{k}-\overline{\mathbf{q}}_{k}\right)\right]
$$

Since $\mathbf{x}_{k}$ and $\mathbf{q}_{k}$ are the only random vectors on the right-hand side and $E\left[\mathbf{x}_{k}\right]=\mathbf{x}_{k}^{a}$ and $\mathrm{E}\left[\mathbf{q}_{k}\right]=\overline{\mathbf{q}}_{k}$, Eq. (62) is reduced to 


$$
\mathbf{x}_{k+1}^{f}=\mathbf{g}_{k}\left(\mathbf{x}_{k}^{a}, \mathbf{f}_{k}, \overline{\mathbf{q}}_{k}\right)
$$

Assuming $\mathrm{E}\left[\left(\mathbf{x}_{k}-\mathbf{x}_{k}^{a}\right)\left(\mathbf{q}_{k}-\overline{\mathbf{q}}_{k}\right)^{T}\right]=0$, the variance $\mathbf{P}_{k+1}^{f}$ is obtained by using Eq. (61) and Eq. (63) as

$$
\begin{aligned}
& \mathrm{E}\left[\left(\mathbf{x}_{k+1}^{f}-\mathrm{E}\left[\mathbf{x}_{k+1}^{f}\right]\right)\left(\mathbf{x}_{k+1}^{f}-\mathrm{E}\left[\mathbf{x}_{k+1}^{f}\right]\right)^{T}\right] \\
& \quad=\mathrm{E}\left[\left(\mathbf{x}_{k+1}^{f}-\mathbf{g}_{k}\left(\mathbf{x}_{k}^{a}, \mathbf{f}_{k}, \overline{\mathbf{q}}_{k}\right)\right)\left(\mathbf{x}_{k+1}^{f}-\mathbf{g}_{k}\left(\mathbf{x}_{k}^{a}, \mathbf{f}_{k}, \overline{\mathbf{q}}_{k}\right)\right)^{T}\right] \\
& \quad=\mathrm{E}\left[\left(\mathbf{A}_{k}\left(\mathbf{x}_{k}-\mathbf{x}_{k}^{a}\right)+\mathbf{B}_{k}\left(\mathbf{q}_{k}-\overline{\mathbf{q}}_{k}\right)\right)\left(\mathbf{A}_{k}\left(\mathbf{x}_{k}-\mathbf{x}_{k}^{a}\right)+\mathbf{B}_{k}\left(\mathbf{q}_{k}-\overline{\mathbf{q}}_{k}\right)\right)^{T}\right] \\
& \quad=\mathrm{E}\left[\left(\mathbf{A}_{k}\left(\mathbf{x}_{k}-\mathbf{x}_{k}^{a}\right)+\mathbf{B}_{k}\left(\mathbf{q}_{k}-\overline{\mathbf{q}}_{k}\right)\right)\left(\left(\mathbf{x}_{k}-\mathbf{x}_{k}^{a}\right)^{T} \mathbf{A}_{k}^{T}+\left(\mathbf{q}_{k}-\overline{\mathbf{q}}_{k}\right)^{T} \mathbf{B}_{k}^{T}\right)\right] \\
& \quad=\mathbf{A}_{k} \mathrm{E}\left[\left(\mathbf{x}_{k}-\mathbf{x}_{k}^{a}\right)\left(\mathbf{x}_{k}-\mathbf{x}_{k}^{a}\right)^{T}\right] \mathbf{A}_{k}^{T}+\mathbf{B}_{k} \mathrm{E}\left[\left(\mathbf{q}_{k}-\overline{\mathbf{q}}_{k}\right)\left(\mathbf{q}_{k}-\overline{\mathbf{q}}_{k}\right)^{T}\right] \mathbf{B}_{k}^{T} \\
& \quad=\mathbf{A}_{k} \mathbf{P}_{k}^{a} \mathbf{A}_{k}^{T}+\mathbf{B}_{k} \mathbf{Q}_{k} \mathbf{B}_{k}^{T}
\end{aligned}
$$

Hence, the forecasting step yields the forecast state pdf

$$
\begin{aligned}
\mathrm{p}\left(\mathbf{x}_{k+1} \mid \mathbf{d}_{1: k}, \boldsymbol{\phi}\right) & =\mathcal{N}\left(\mathbf{x}_{k+1} \mid \mathbf{x}_{k+1}^{f}, \mathbf{P}_{k+1}^{f}\right) \\
& =\mathcal{N}\left(\mathbf{x}_{k+1} \mid \mathbf{g}_{k}\left(\mathbf{x}_{k}^{a}, \mathbf{f}_{k}, \overline{\mathbf{q}}_{k}\right), \mathbf{A}_{k} \mathbf{P}_{k}^{a} \mathbf{A}_{k}^{T}+\mathbf{B}_{k} \mathbf{Q}_{k} \mathbf{B}_{k}^{T}\right)
\end{aligned}
$$

This completes the forecast step. The overall EKF procedure is summarized in Fig. 7.

Since $\mathrm{p}\left(\mathbf{d}_{k} \mid \mathbf{d}_{1: k-1}, \boldsymbol{\phi}\right)=\mathcal{N}\left(\mathbf{d}_{k} \mid \mathbf{h}_{k}\left(\mathbf{x}_{k}^{f}, \bar{\epsilon}_{k}\right), \mathbf{C}_{k} \mathbf{P}_{k}^{f} \mathbf{C}_{k}^{T}+\mathbf{D}_{k} \boldsymbol{\Gamma}_{k} \mathbf{D}_{k}^{T}\right)$ from Eq. (54), we can simplify Eq. (44b) as

$$
\mathrm{p}\left(\phi \mid \mathbf{d}_{1: n}\right) \propto \mathrm{p}(\phi) \prod_{k=1}^{n} \mathrm{p}\left(\mathrm{d}_{k} \mid \mathbf{d}_{1: k-1}, \phi\right)
$$




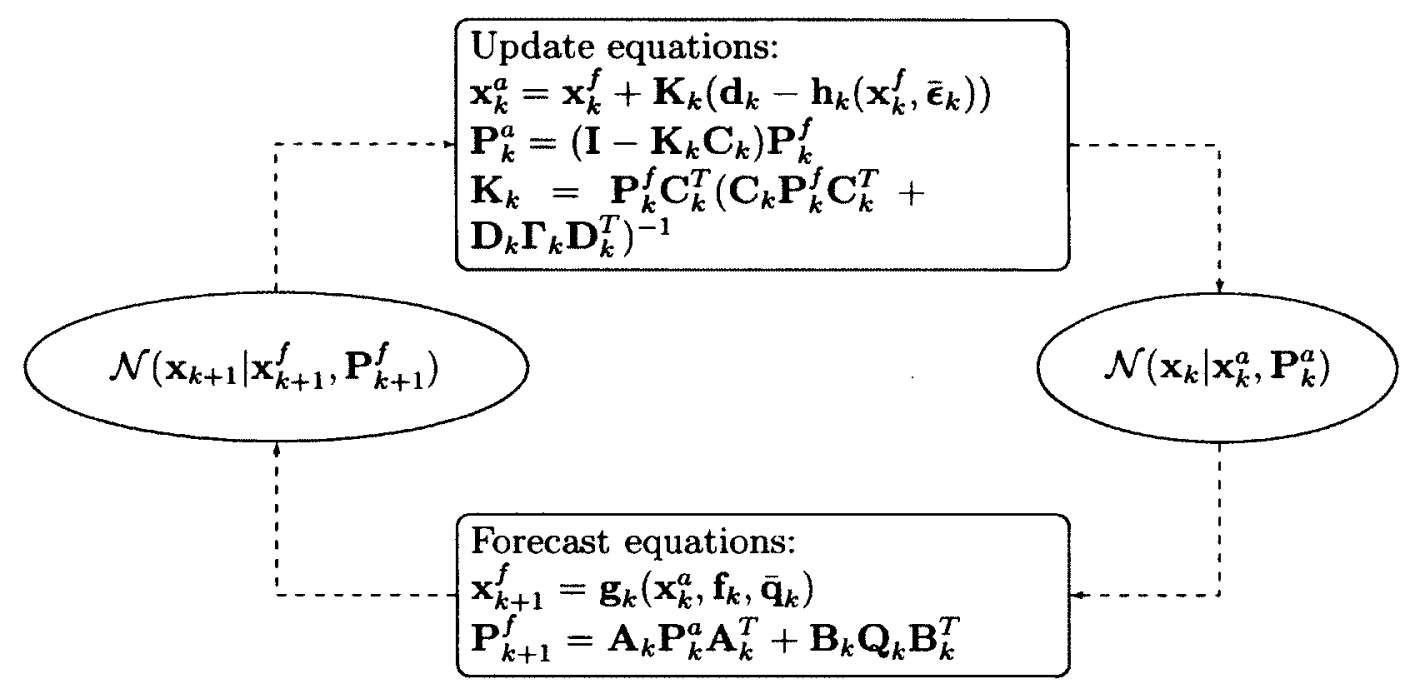

Figure 7: Summary of the EKF procedure

$$
\propto \mathrm{p}(\boldsymbol{\phi}) \prod_{k=1}^{n} \mathcal{N}\left(\mathbf{d}_{k} \mid \mathbf{h}_{k}\left(\mathbf{x}_{k}^{f}, \overline{\boldsymbol{\epsilon}}_{k}\right), \mathbf{C}_{k} \mathbf{P}_{k}^{f} \mathbf{C}_{k}^{T}+\mathbf{D}_{k} \boldsymbol{\Gamma}_{k} \mathbf{D}_{k}^{T}\right)
$$

For a given model $\mathcal{M}$ and data $\mathbf{d}_{1: n}$, the right-hand side of Eq. (66b) is only a function of $\phi$ and, therefore, defines the curvature of the posterior distribution. Next, we discuss the MCMC method employed to sample from the posterior distribution in Eq. (66b).

\subsection{Monte Carlo Markov Chain sampling algo- rithm}

MCMC methods $[69,82]$ are a class of algorithms that simulate random samples from complex probability distributions by constructing a Markov chain whose stationary distribution is the desired distribution. Unlike simple Monte Carlo sampling methods, MCMC methods do not require the normalizing constant of the targeted distribution 
to be known. This special property of MCMC methods find their widespread use in Bayesian inference for sampling posterior distributions like Eq. (66b). MCMC methods are also popular due to their ease of implementation in sampling complex distributions where traditional methods fail. Next, we discuss the properties of a stationary Markov chain that we seek to generate through MCMC methods.

\subsubsection{Markov Chains}

A Markov chain is a special type of discrete-time stochastic process denoted by a sequence of random vectors $\left\{\mathbf{X}_{0}, \mathbf{X}_{1}, \ldots\right\}$ that take, say, $\mathbf{x}_{k}$ value at state $\mathbf{X}_{k}$. A firstorder Markov chain is generated such that any future state $\mathbf{X}_{k+1}$ will only depend on the current state $\mathbf{X}_{k}$ and is independent of the past state history $\mathbf{X}_{1: k-1}[69,82]$. In mathematical terms [69],

$$
\mathrm{p}\left(\mathbf{X}_{k+1}=\mathbf{x}_{k+1} \mid \mathbf{X}_{k}=\mathbf{x}_{k}, \ldots, \mathbf{X}_{0}=\mathbf{x}_{0}\right)=\mathrm{p}\left(\mathbf{X}_{k+1}=\mathbf{x}_{k+1} \mid \mathbf{X}_{k}=\mathbf{x}_{k}\right)
$$

The pdf $\mathrm{p}\left(\mathbf{X}_{k+1}=\mathbf{x}_{k+1} \mid \mathbf{X}_{k}=\mathbf{x}_{k}\right)$ in Eq. (67) is called the transition pdf (kernel). The Markov chain homogeneous if the transition kernel $\mathrm{p}\left(\mathbf{X}_{k+1}=\mathbf{x}_{k+1} \mid \mathbf{X}_{k}=\mathbf{x}_{k}\right)$ does not change with time, i.e for all $k$ it satisfies following property: [69]

$$
\mathrm{p}\left(\mathbf{X}_{k+1}=\mathbf{x}_{k+1} \mid \mathbf{X}_{k}=\mathbf{x}_{k}\right)=\mathrm{p}\left(\mathbf{X}_{k}=\mathbf{x}_{k} \mid \mathbf{X}_{k-1}=\mathbf{x}_{k-1}\right)=A(\mathbf{y}, \mathbf{z}) .
$$

The transition kernel should always satisfy following:

$$
A(\mathbf{y}, \mathbf{z}) \geq \forall \mathbf{y}, \mathbf{z} \in S \quad \text { and } \quad \int A(\mathbf{y}, \mathbf{z}) d \mathbf{z}=1
$$

In the context of MCMC sampling, we will only consider the first-order, homogeneous Markov chains in this thesis. 
As the Markov chain progresses from a starting state $X_{0}$, the distribution of subsequent states starts converging to a unique stationary distribution $\pi($.$) provided$ the chain satisfies following properties $[69,82]$ :

- Irreducibility : A irreducible Markov chain can move from any starting state to any other state with some positive probability. This movement can be in one step or multiple steps.

- Aperiodicity : An aperiodic Markov chain does not oscillate between any two states.

- Positive recurrent : If a Markov chain samples from the stationary distribution $\pi($.$) at some point of time, all subsequent samples of a positive recurrent$ chain will be distributed according to $\pi($.$) . A positive recurrent chain follows$ the relation

$$
\int \pi\left(\int \pi(\mathbf{y}) A(\mathbf{y}, \mathbf{z}) d \mathbf{y}=\pi(\mathbf{z})\right.
$$

The stationary distribution $\pi($.$) of the chain that satisfies the above three properties$ will be independent of $X_{0}$ and ' $k$ ' values $[69,82]$. This implies

$$
\mathrm{p}\left(\mathbf{X}_{k} \mid \mathbf{X}_{0}\right) \rightarrow \pi\left(\mathbf{X}_{k}\right) \text { as } k \rightarrow \infty
$$

In practice, the value of $k$ after which the chain converges to the stationary distribution $\pi($.$) is called as the burn-in period [69,82]$. Here, the convergence of the chain means that the samples are independent of the starting point and look more like dependent samples from the stationary distribution.

In the parameter estimation, our aim through MCMC sampling is to generate an 
irreducible, aperiodic and positive-recurrent Markov chain whose stationary distribution $\pi($.$) is the distribution we want to sample from (i.e. Eq. (66b)). Since, the$ stationary distribution is already known, we seek the transition kernel $\mathrm{p}\left(X_{k+1} \mid X_{k}\right)$ which can generate such a Markov chain, given that the distribution is only known up to a constant of proportionality.

\subsubsection{Metropolis-Hastings algorithm}

Metropolis-Hastings (M-H) algorithm is the most popular MCMC algorithm employed for generating a Markov chain whose stationary distribution $\pi($.$) is the target$ distribution $[68,69]$. It was first proposed by Metropolis et al. [83] and was later generalized by Hastings [84]. The widespread use of the $\mathrm{M}-\mathrm{H}$ algorithm is attributed to its ability to sample from complex pdf using simple transition kernels. This property is exploited in Bayesian inference where little is known about the potentially complex features of the posterior distribution due to its dependence on the quantity and quality of the measurement data.

The $\mathrm{M}-\mathrm{H}$ algorithm operates by first proposing a future candidate move $\mathbf{y}$ from the current state $\mathbf{z}$ of the Markov chain using a time-invariant proposal pdf $q(\mathbf{y}, \mathbf{z})$. The proposed candidate is then accepted as a future move of the Markov chain with probability $\alpha(\mathbf{y}, \mathbf{z})$ where $[68,69]$

$$
\alpha(\mathbf{y}, \mathbf{z})=\min \left(1, \frac{\pi(\mathbf{z})}{\pi(\mathbf{y})} \frac{q(\mathbf{z}, \mathbf{y})}{q(\mathbf{y}, \mathbf{z})}\right)
$$

The transition kernel is obtained as $[68,69]$

$$
A(\mathbf{y}, \mathbf{z})=\alpha(\mathbf{y}, \mathbf{z}) q(\mathbf{y}, \mathbf{z})
$$


The transition kernel $A(\mathbf{y}, \mathbf{z})$ is derived by satisfying the detailed balance condition of the transition from $\mathbf{y}$ to $\mathbf{z}[68]$. The detailed balance condition implies $[68,69]$

$$
\begin{aligned}
\pi(\mathbf{y}) A(\mathbf{y}, \mathbf{z}) & =\pi(\mathbf{z}) A(\mathbf{z}, \mathbf{y}) \\
\pi(\mathbf{y}) \alpha(\mathbf{y}, \mathbf{z}) q(\mathbf{y}, \mathbf{z}) & =\pi(\mathbf{z}) \alpha(\mathbf{z}, \mathbf{y}) q(\mathbf{z}, \mathbf{y})
\end{aligned}
$$

If for some $\pi$, the transition kernel satisfies the detailed balance equation, the Markov chain thereby generated will have a stationary distribution $\pi$ and will be reversible with respect to $\pi($.$) (See Chib and Greenberg [68] or Gilks et al. [69] for detailed$ regularity conditions and proofs regarding Markov chain properties.

There are several striking properties of the $\mathrm{M}-\mathrm{H}$ algorithm. First, as seen in Eq. (72), the M-H algorithm does not require the normalizing constant of the target distribution $\pi($.$) . Second, there are no assumptions about the proposal pdf q(Y \mid X)$. This means that any proposal pdf $q(Y \mid X)$ in $\mathrm{M}-\mathrm{H}$ algorithm will generate an irreducible, aperiodic and positive-recurrent Markov chain that will converge to a stationary distribution $\pi($.$) in a finite number of iterations, irrespective of the starting$ point $[68,69]$.

Inspite of these useful properties and the simplicity of implementation ease of the M-H algorithm, the computational efficiency of the MCMC algorithm is severely influenced by the choice of the proposal pdf. This is a well-known and well-researched issue $[69,72,74,76,77]$. In this thesis, we resolve this issue by adopting an adaptive version of the $\mathrm{M}-\mathrm{H}$ algorithm, which is discussed next.

\subsubsection{An Adaptive Random Walk Metropolis algorithm}

The most commonly used form of the $\mathrm{M}-\mathrm{H}$ algorithm is the Random-Walk Metropolis (RWM) algorithm, for which the proposal is symmetric and always centered at the 
current position of the chain $[69,72,74,76,77]$. In this thesis, we will use gaussian proposal distribution for implementing RWM algorithm to sample parameter $\phi$ from complex, multi-dimensional posterior distributions $\mathrm{p}(\phi \mid \mathbf{d})$ of the type shown in Eq. (66b). In other words, the proposal $q\left(\phi, \phi^{\prime}\right)=\mathcal{N}(\phi, \Sigma)$ will be used for proposing a new move $\phi^{\prime}$ from the current position $\phi$ of the Markov chain whose stationary distribution $\pi($.$) is \mathrm{p}(\boldsymbol{\phi} \mid \mathbf{d})$.

In RWM algorithm, the proposal pdf $\mathcal{N}(\phi, \Sigma)$ is always centered at the current position $\phi$ whose spread is determined by the covariance matrix $\Sigma$. The probability that the move $\phi^{\prime}$ is accepted can be obtained from Eq. (72) as

$$
\alpha\left(\phi, \phi^{\prime}\right)=\min \left(1, \frac{\mathrm{p}\left(\boldsymbol{\phi}^{\prime} \mid \mathbf{d}\right)}{\mathrm{p}(\boldsymbol{\phi} \mid \mathbf{d})}\right)
$$

As discussed earlier, the covariance $\boldsymbol{\Sigma}$ will play an important role in determining the rate of the convergence of the Markov chain. For a fixed target distribution, $\boldsymbol{\Sigma}$ needs to be scaled to ensure better mixing of the chain.

We demonstrate the importance of proposal scaling in MCMC sampling by taking a simple example. For instance, when sampling $\phi$ from a target distribution $\phi \sim$ $\mathcal{N}(0,1)$ by a proposal $\mathcal{N}\left(\phi, w^{2}\right)$, different values of $w$ will result different mixing properties of the MCMC chain. Fig. 8 shows the comparison of MCMC chains for $w$ values of $0.1,1.0$ and 10.0 .

The proposal with less spread $(w=0.1)$ than the target distribution $(\mathcal{N}(0,1)$ in this case) will propose smaller jumps from the current position resulting in most of the proposed moves being accepted (Acceptance ratio $=97.1 \%$ ). The higher acceptance ratio results in chain taking longer time to explore the posterior space and, therefore, taking longer time to converge. On the other hand, the proposal with larger spread ( $w=10.0$ ) will propose large jumps to regions with low posterior probability resulting 

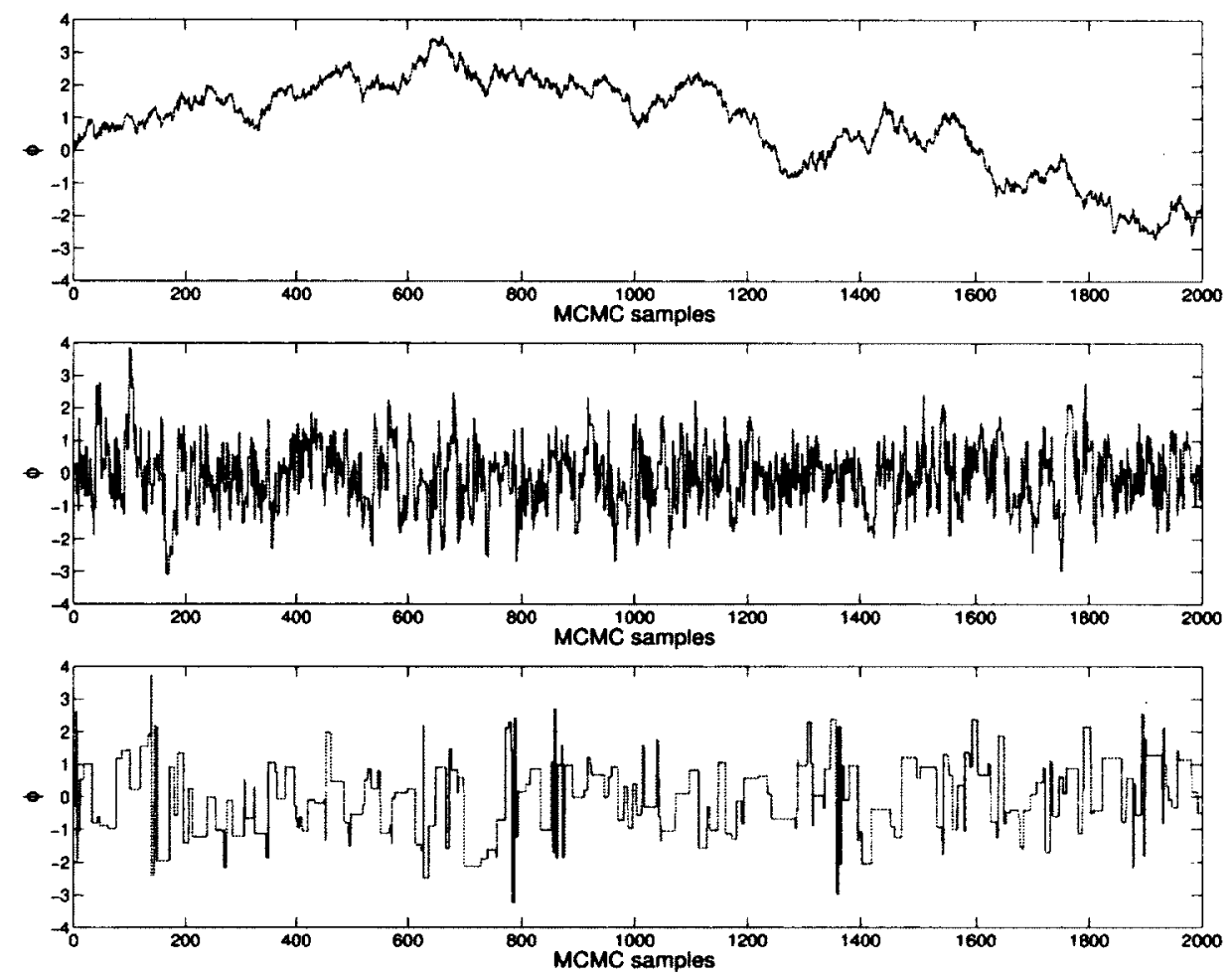

Figure 8: Comparison of MCMC chains generated with $w$ values of $0.1,1.0$ and 10.0 (in that order, from top to bottom). All the three chain have same starting position $(\phi=0.0)$ and chain length (2000). 
in most of the proposed moves being rejected (Acceptance ratio $=11.4 \%$ ). The lower acceptance ratio results in chain remaining at the current position for large numbers of iterations. The proposal with $w=1.0$ results in better mixing than the cases of $w=0.1$ and $w=10.0$. It is clear that their exists an optimal value of $w$ for a given target distribution for which the mixing of the Markov chain will be best, if not perfect. This is in general true for any target distribution when using RWM algorithm $[69,72,74,76,77]$.

In the current parameter estimation scenario, we are sampling $p$-dimensional $\phi$ ( $p$ is roughly $5-22$ in this thesis) from a complex posterior $\mathrm{p}(\phi \mid \mathrm{d})$. Given that we have very little prior information about the covariance structure of $\phi$, the manual tuning of $\boldsymbol{\Sigma}$ is not an efficient approach. Also, since we are dealing with large number of models in the process of model selection, the task of manual tuning will be quite overwhelming. It is, however, clear that for a given posterior $\mathrm{p}(\phi \mid \mathbf{d})$, there will be an optimal $\boldsymbol{\Sigma}$ that will result in the fastest convergence of the chain.

In this thesis, we resort to an Adaptive Proposal RWM (AP-RWM) method [77] to alleviate the need of assigning a good $\Sigma$ in order to sample the posterior in Eq. (66b). The AP-RWM algorithm automatically tunes an initial badly proposed $\boldsymbol{\Sigma}$ by learning about the posterior from the past history of the chain to result in a 'better' $\boldsymbol{\Sigma}$. Here, 'better' implies good mixing and faster convergence of the chain to the posterior distribution.

Consider that a $p$-dimensional parameter $\phi$ is being sampled from a posterior $\mathrm{p}(\phi \mid \mathbf{d})$ in Eq. (66b). Before initializing MCMC sampling, we fix a memory parameter $W$ that controls the size of the chain length which is used to update $\boldsymbol{\Sigma}$. Hence, at any update step, $W$ number of posterior samples are available as $\left\{\phi^{(1)}, \phi^{(2)}, \ldots, \phi^{(W)}\right\}$. These posterior samples are first compiled in a matrix $R$ of size $p \times W$. The updated $\Sigma$ is then obtained using the MCMC samples as follows: [77] 


$$
\Sigma=s_{d}\left\{\frac{1}{W-1}[R-\mathrm{E}[R]]^{T}[R-\mathrm{E}[R]]\right\}
$$

The expectation $E[$.$] in the above expression is taken in the row direction. The$ expected value is then subtracted from the entire corresponding row. The constant $s_{d}=2.38^{2} / p$ [72] in Eq. (76) is derived under asymptotic conditions of $p \rightarrow \infty$ for sampling from gaussian distributions. The corresponding acceptance ratio is 23.4 $\%$ [72]. Fig. 9 contrasts the performance of AP-RWM algorithm with the RWM algorithm for the case of poor proposal scale and starting point for sampling from $\mathcal{N}(0,1)$

The tuning of $\boldsymbol{\Sigma}$ is stopped after the burn-in is reached. The condition for the burn-in period is two fold. One, we make sure that the mode of the posterior is not changing for a long period of the chain while the MCMC sampling continues. Second, we make sure that $\boldsymbol{\Sigma}$ is not changing significantly by tracking the rejection ratio of a $W$ length of the generated chain. We keep the memory parameter $W$ large enough to have less variations in $\mathbf{\Sigma}$ in subsequent tuning after the chain converges to the posterior. Once both the conditions for the burn-in is reached, we stop the tuning and the samples are generated using a constant $\boldsymbol{\Sigma}$. The overall MCMC procedure untill the burn-in period is summarized in Fig. 10 .

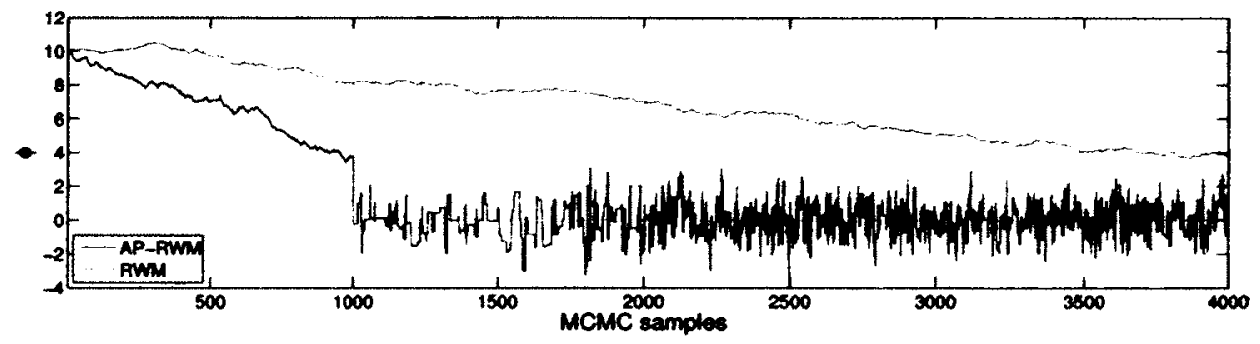

Figure 9: Comparison of MCMC chains generated with fixed starting values of $w=$ 0.05 and $\phi=10$ 


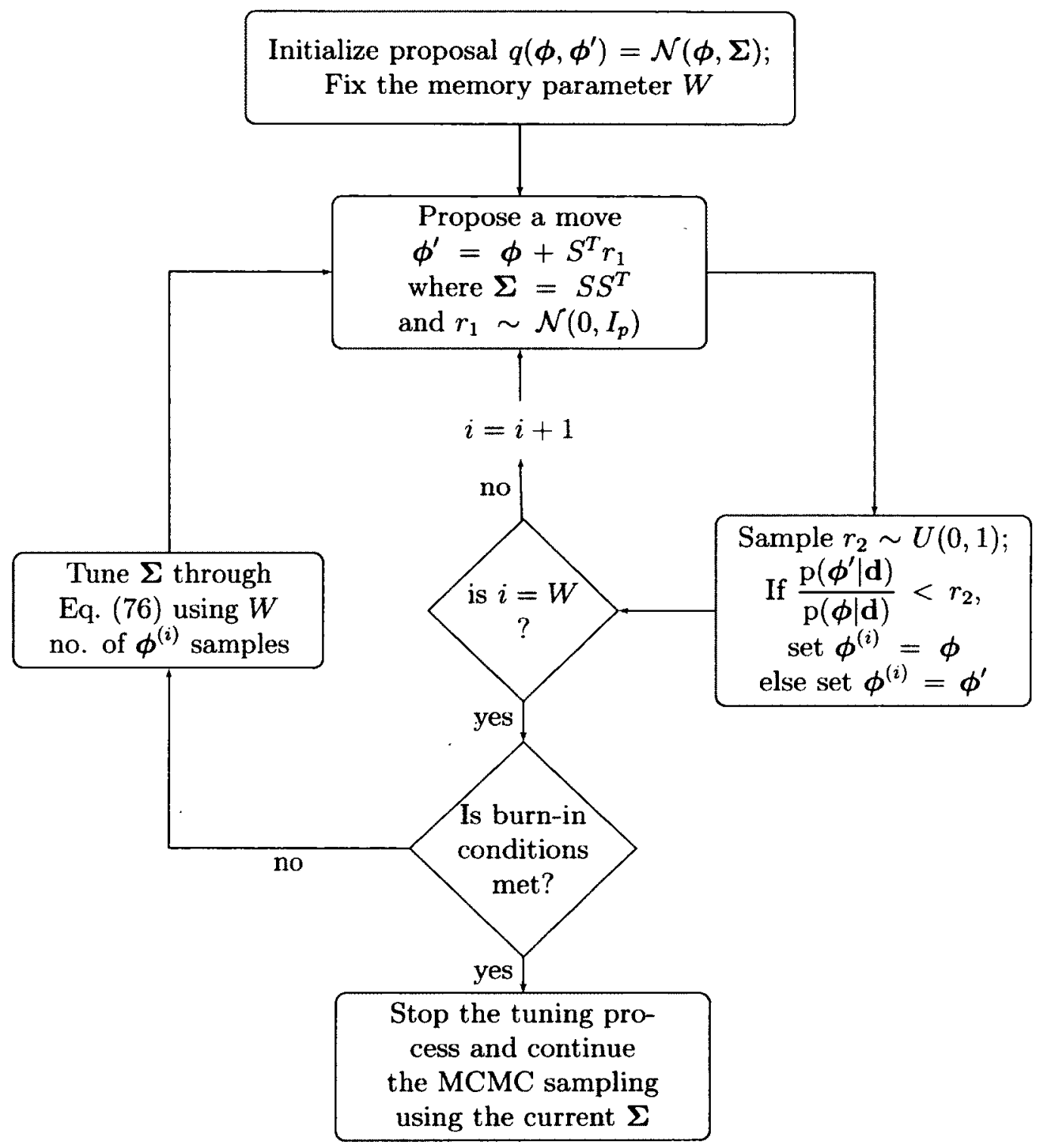

Figure 10: Summary of the MCMC procedure untill the burn-in period 
No tuning is performed after the burn-in period in order to maintain the Markovian property of the chain. The Markov chain, generated after burn-in, has its stationary distribution same as the posterior. The output of this Markov chain, combined from all processors, is then used to estimate the posterior pdf and calculating the marginal likelihood for model selection. The parallel implementation of the sampling algorithm is done to generate large number of samples in minimum time, thereby expediting the model selection process.

\subsection{Maximum Likelihood Estimation}

If we are assigning non-informative priors $\mathrm{p}(\phi) \propto 1)$ to all the models, the posterior can be re-written from $\mathrm{Eq}$. (66b) as

$$
\begin{aligned}
\mathrm{p}\left(\phi \mid \mathbf{d}_{1: n}\right) & \propto \mathrm{p}\left(\mathbf{d}_{1: n} \mid \phi\right) \propto \prod_{k=1}^{n} \mathrm{p}\left(\mathbf{d}_{k} \mid \mathbf{d}_{1: k-1}\right) \\
& \propto \prod_{k=1}^{n} \mathcal{N}\left(\mathbf{d}_{k} \mid \mathbf{h}_{k}\left(\mathbf{x}_{k}^{f}, \overline{\boldsymbol{\epsilon}}_{k}\right), \mathbf{C}_{k} \mathbf{P}_{k}^{f} \mathbf{C}_{k}^{T}+\mathbf{D}_{k} \boldsymbol{\Gamma}_{k} \mathbf{D}_{k}^{T}\right)
\end{aligned}
$$

In this case, the mode $\hat{\phi}_{M A P}$ of the posterior $\mathrm{p}\left(\phi \mid \mathbf{d}_{1: n}\right)$ will be same as the MLE estimate $\hat{\phi}$, obtained as

$$
\hat{\boldsymbol{\phi}}_{M A P}=\hat{\boldsymbol{\phi}}=\operatorname{Max}\left\{\prod_{k=1}^{n} \mathcal{N}\left(\mathbf{d}_{k} \mid \mathbf{h}_{k}\left(\mathbf{x}_{k}^{f}, \overline{\boldsymbol{\epsilon}}_{k}\right), \mathbf{C}_{k} \mathbf{P}_{k}^{f} \mathbf{C}_{k}^{T}+\mathbf{D}_{k} \boldsymbol{\Gamma}_{k} \mathbf{D}_{k}^{T}\right)\right\}
$$

In order to find $\hat{\phi}$, we optimize the posterior $\mathrm{p}(\phi \mid \mathbf{d})$ using the proposed adaptive sampling algorithm in conjunction with a line search optimization technique. This optimization step is further expedited by its distributed implementation using Message Passing Interface (MPI) to exploit the HPC systems. The expedition is performed in two ways: 1) the starting value of the MLE estimate for the optimization is different 
for every core, each independently sampled from a gaussian distribution centered at the initial MLE estimate provided by the user, and 2) after certain interval of time, the MLE estimate is compared over all the cores and the best MLE is communicated to all the cores. This synchronous parallel optimization technique has a higher chances of finding the MLE estimate in a less time than its serial counterpart. 


\section{Chapter 5}

\section{Application: A fluid-structure interaction problem}

In this chapter, we apply the proposed system identification scheme to model a nonlinear aeroelastic oscillator consisting of a wing performing low amplitude LCO in the transitional Reynolds number (Re) regime. Firstly, we describe the wind-tunnel set-up of the oscillator and the characteristics of the oscillations. Then, we perform a numerical study to validate the proposed system identification scheme by mimicking the free decay and the LCO response acquired from the wind-tunnel. Finally, we present and discuss the results for the system identification of the oscillator for four different Reynolds number.

\subsection{The nonlinear aeroelastic oscillator}

\subsubsection{The wind-tunnel set-up}

The aeroelastic apparatus where the oscillator is situated is a symmetric and rigid NACA0012 wing attached in a low speed wind tunnel at the Royal Military College of 
Canada (RMC). The wind tunnel is a closed circuit and is powered by a $75 \mathrm{~kW}$ threephase motor. It has a test section of $0.76 \mathrm{~m} \times 1.08 \mathrm{~m}$ and is capable of generating airspeeds ranging from 5 to $60 \mathrm{~m} / \mathrm{s}$. The maximum turbulence intensity was observed to be less than $0.2 \%$ for the airspeeds $(7.5$ to $10.5 \mathrm{~m} / \mathrm{s})$ considered in this exercise. A pitot-static tube located at the inlet of the test section is used for measuring the airspeed. Fig. 11 shows the layout (taken from Poirel et al. [85]) of the wind-tunnel.

** Removed due to the copyright issue ${ }^{* *}$

Figure 11: Layout of the wind-tunnel
Removed due to the copyright issue**

Figure 12: Schematic of the wing apparatus

The NACA0012 wing installed in the wind tunnel undergoes plunge (translation) and pitch (rotation). The wing is installed in the vertical plane to nullify the effects of gravity on the targeted physics of the problem. Fig. 13 shows a photograph (taken from Poirel et al. [85]) of the wing installed in the test section. The bottom and the top of the wing surface is attached to a similar translating sub-system through a 0.015 diameter fiberglass rod. The position of the rod can be varied along the chord to adjust the position of the axis of rotation or the elastic axis (EA). Each translating subsystem consists of na interconnected set of circular pulleys and translational springs hoisted on a rectangular plate. The sub-system serves two purposes: 1) it provides the elastic restoring force to both pitch and plunge motion, and 2) converts the pitch motion into a translational spring elongation. The top and bottom sub-systems are connected by a vertical rod, thereby restricting the rectangular plates to move as a single unit. More detailed description of the wind-tunnel apparatus can be found in Poirel et al. [86].

The wing has a uniform cross-section in the horizontal plane with a span $s=0.61 \mathrm{~m}$ and a chord length $c=0.156 \mathrm{~m}$, resulting in an aspect ratio (AR) of 3.9. The gap between the wing tips and the end plates, which are introduced to minimize the 3D 
effects, is $7 \mathrm{~mm}$ ( $1 \%$ of the wing span). The elastic axis is located at $18.6 \%$ chord behind the leading edge of the wing. Since only pure pitch motion is considered in this investigation, the mass ratio is calculated as $\left.\mu=m /\left(\rho \pi(c / 2)^{2} s\right)\right)=55.6$. The pitch displacement is measured using a rotary potentiometer and then recorded through a LabVIEW based data acquisition system. Fig. 14 shows the $2 \mathrm{D}$ representation of the wing apparatus, to be used for modelling the wing motion. $a_{h}$

**Removed due to the copyright issue**

Figure 13: Photograph of the wing apparatus

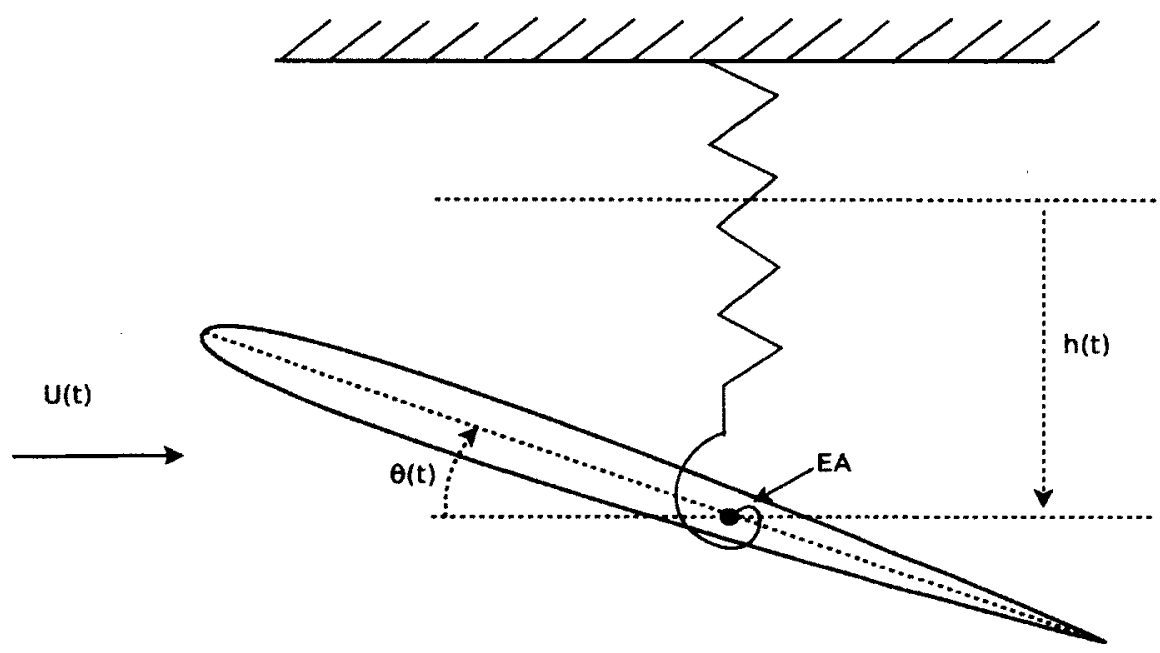

Figure 14: $2 \mathrm{D}$ representation of the wing system

\subsubsection{The aeroelastic oscillations}

When the plunge dof is held fixed and the airflow is in the transitional Re range of $5.0 \times 10^{4} \leq \operatorname{Re}_{c} \leq 1.3 \times 10^{5}\left(\operatorname{Re}_{c}\right.$ is the chord-based $\left.R e\right)$, the NACA0012 wing losses its aeroelastic stability and starts performing pure pitch oscillations about the equilibrium position of $\theta=0$. These oscillations were first reported and studied by Poirel et al. [87]. The pitch response measured at four different Re values with 
a sampling frequency of $1000 \mathrm{~Hz}(\Delta t=0.001 \mathrm{~s})$ is shown in Fig. 15. The loss of stability was triggered by a small external perturbation, either given to the wing or to the airflow. Note that the oscillations are not sustained outside the aforementioned Re range. More detailed analysis of the sensitivity of the initial conditions and the flow perturbations to the existence and sustainability of the oscillations is given in Poirel et al. [87]. The observed sensitivity of the oscillations to the surface roughness, the free-stream turbulence intensity and the airspeed variations suggests the role of the boundary layer transition in governing the nonlinear aeroelastic dynamics of the oscillations [87].

Unlike stall flutter where the high angle-of-attack (AOA) is the primary reason for the flow separation and the subsequent aeroelastic instability, flows in the transitional Re range of $10^{4} \leq \operatorname{Re}_{c} \leq 10^{5}$ are well known to exhibit complex flow separation aerodynamics for angles well below the stall flutter [3, 88-92]. The complex boundary layer characteristics such as laminar boundary layer separation, transition of the laminar shear layer and re-attachment of the turbulent boundary layer leading to the formation of a Laminar separation bubble (LSB) are typical of the transitional Re regime and are well known in the literature [88, 90-92]. Such a complex viscous phenomenon severely effects the pressure distribution over the wing surface, resulting in nonlinear aerodynamic forces being applied to the wing [3, 88, 89].

Huang and Lee [93] conducted a surface-oil analysis on a statically held cantilever NACA0012 wing and found following observations : laminar boundary layer separation at $2^{\circ}$, formation of a LSB between $2^{\circ}$ and $13^{\circ}$ approximately and turbulent trailing edge separation for angles greater than $8^{\circ}$. Using the same apparatus, Huang et al. [94] observed the nonlinearity in static aerodynamic moment coefficient about the quarter-chord length for varying AOA. Fig. 16 shows the coefficient values obtained from Huang et al. [94] for $\operatorname{Re}=77,000$ and $R e=100,000$. Several authors 


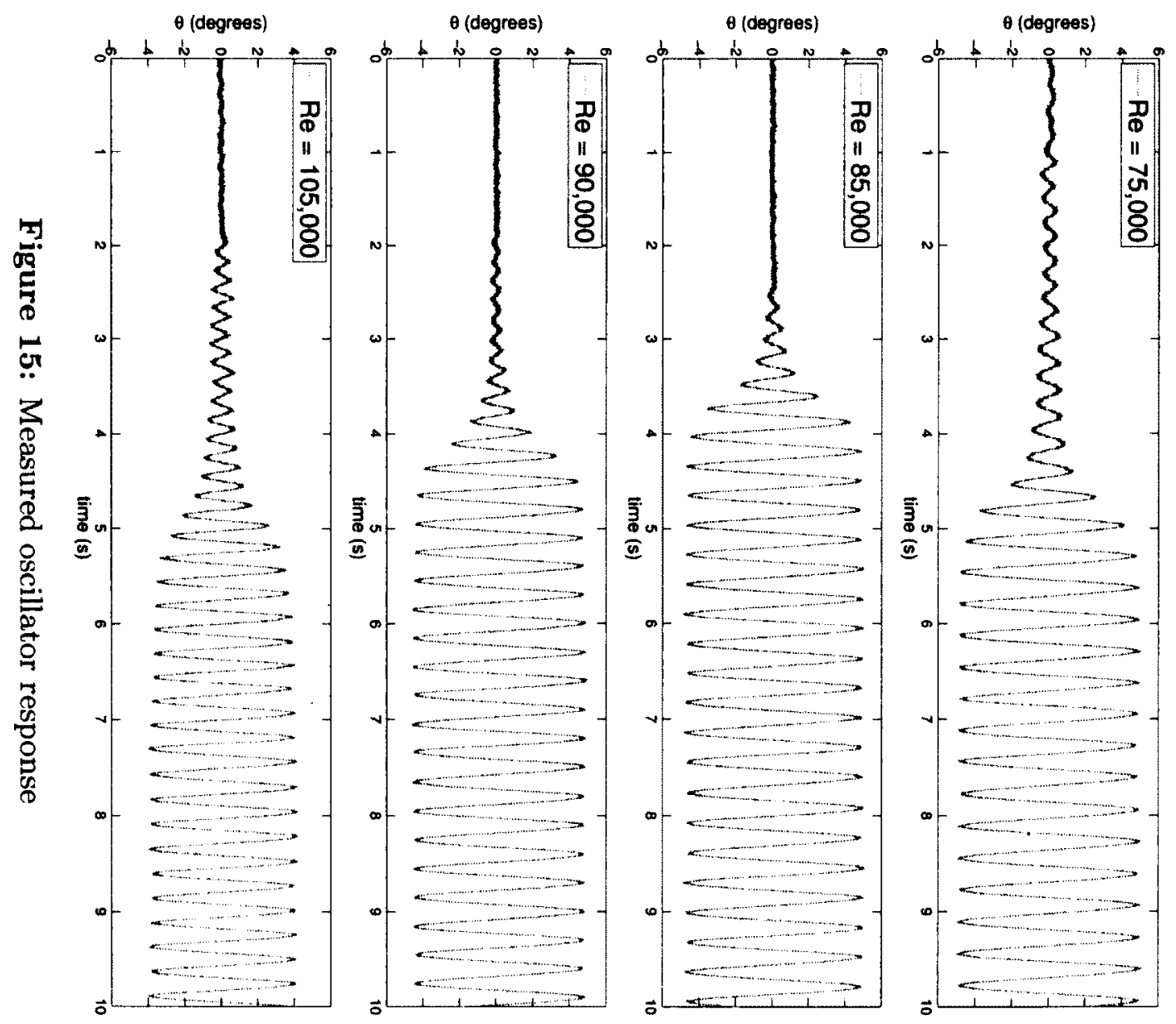


have studied this complex flow separation aerodynamics and have obtained similar conclusions $[3,88-92]$.

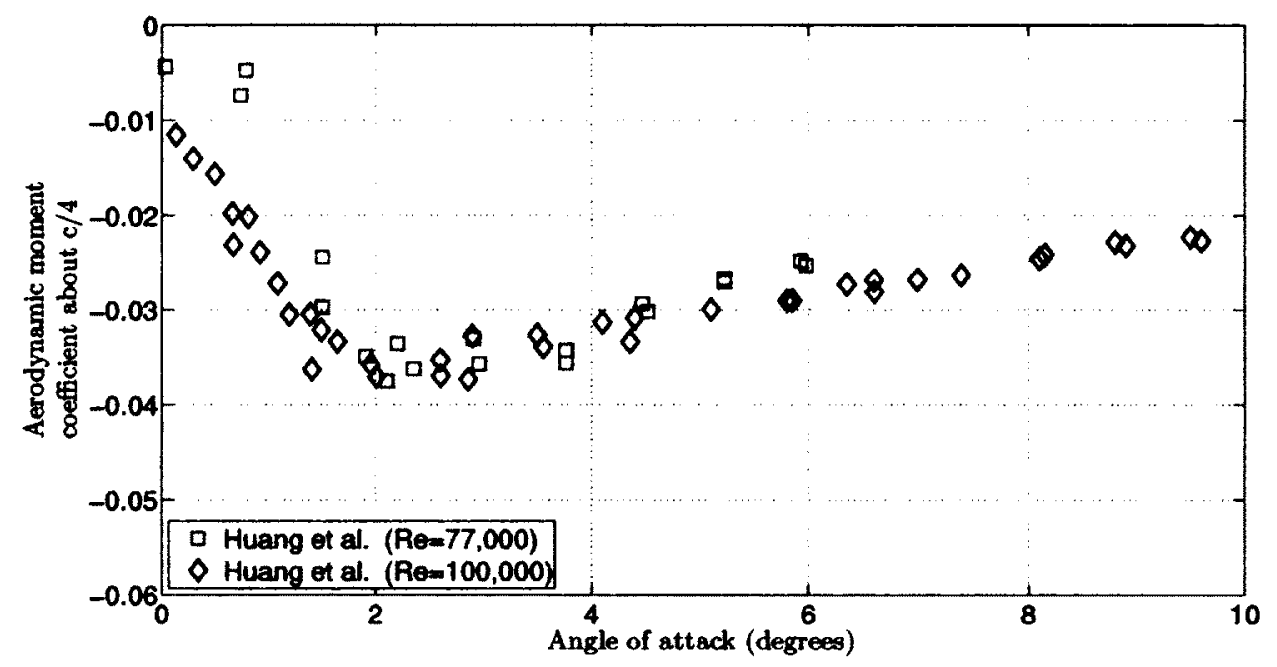

Figure 16: Static aerodynamic moment coefficient about $c / 4$

Recently proved through large eddy simulations by Poirel and Yuan [3] and URANS calculations by Poirel et al. [85], the aforementioned viscous phenomenon plays an important part in governing the dynamics of the oscillations shown in Fig. 15. The aeroelastic instability of the wing is caused by the laminar trailing-edge separation creating negative aerodynamic damping greater than the linear structural damping [3]. For the same reason, the dynamic instability is also termed as the laminar separation flutter. The role of laminar trailing-edge separation is reinforced by the fact that the instability vanishes when the upstream flow is disturbed by placing a turbulence-generating grid [3]. The re-stabilization to LCO is attributed to the presence of the nonlinear aerodynamic damping acting against the negative aerodynamic damping. This nonlinear aerodynamic damping and its variation with $R e$ is attributed to the presence of LSB and turbulent trailing edge separation [3]. The lag in the position of LSB on the the top and bottom surfaces of the wing feeds the LCO.

The growing number of operating vehicles like the Unmanned Aerial Vehicles 
(UAVs) and the Micro Air Vehicles (MAVs) flying in the transitional Re regime has encouraged engineers to investigate the nonlinear flow separation aerodynamics that occurs in these special flow conditions and its effect on the lifting and aeroelastic stability characteristics of the lifting body (wing). The oscillations shown in Fig. 15 is a perfect example where the wing performance is severely affected by the nonlinear aerodynamics. In this thesis, we aim to understand the nonlinear aerodynamic forcing and its variation with the AOA and Re values by modelling the measured oscillator response.

\subsubsection{Quasi-steady aerodynamics}

Generally speaking, the aerodynamic forces acting on the wing shown in Fig. 14 can be modeled as

$$
\text { Aerodynamic forces }=f(\dot{U}, \dot{h}, \ddot{h}, \theta, \dot{\theta}, \ddot{\theta}, t)
$$

where $h$ is the plunge displacement, $\theta$ is the pitch displacement, $U$ is the airspeed and $t$ is the time period. The terms in Eq. (79) can be grouped based on their physical representation as follows: 1) Steady and quasi-steady aerodynamics: $U, \dot{h}, \theta, \dot{\theta}, 2)$ Unsteady aerodynamics due to fluid inertia (non-circulatory): $\ddot{h}, \dot{\theta}, \ddot{\theta}$, and 3) Unsteady aerodynamics due to trailing vortices (Circulatory): $t$.

For the wing apparatus considered in this work, the unsteady effects due to the fluid inertia can be neglected on the ground that the density of the wing is much larger than the fluid (air in this case). Also, the following evidences exist against the influence of the unsteady aerodynamics due to the trailing vortices on the LCO: 1) the flow disturbance in the wake has little effect on the LCO [3], and 2) the frequency of the LCO $(\sim 3 \mathrm{~Hz})$ is much higher than the wake frequency $(\sim 250 \mathrm{~Hz})[3]$. Inspite of these evidences, the effect of the trailing vortices on the wing motion cannot be 
confidently excluded and remains a question of concern. Our primary focus in this work is on system identification of the oscillator assuming quasi-steady aerodynamics. The unsteady aerodynamics will be briefly investigated at the end of this chapter.

The quasi-steady aerodynamics assumes that the airflow adjusts instantaneously to the wing motions and, therefore, the effects of the airflow acceleration and lag/delay are neglected. In other words, the aerodynamic characteristics of an airfoil moving with nonzero acceleration are assumed equal to the same airfoil moving with a constant velocity equal to the velocity of the airfoil at that instant. Assuming quasisteady aerodynamics for the pure pitch $(h=\dot{h}=\ddot{h}=0)$ oscillations considered in this work, the aerodynamic moment $M_{E A}$ about the elastic axis can be modeled as

$$
\mathrm{M}_{E A}=f(\theta, \dot{\theta})
$$

A notable example of a quasi-steady, nonlinear aerodynamic damping model results in a LCO is the van der pol oscillator:

$$
I_{E A} \ddot{\theta}+D_{s} \dot{\theta}+K_{s} \theta=\mathrm{M}_{E A}=f(\theta, \dot{\theta})=K_{a} \theta+D_{a} \dot{\theta}+D_{a}^{\prime} \theta^{2} \dot{\theta}
$$

where $I_{E A}, D_{s}$ and $K_{s}$ are the structural parameters while $K_{a}, D_{a}$ and $D_{a}^{\prime}$ are the aerodynamic parameters.

A general representation of a quasi-steady model for a pure pitch oscillation of the NACA0012 wing can be written as

$$
\begin{aligned}
I_{E A} \ddot{\theta}+f_{s}(\theta, \dot{\theta}) & =f_{a d}(\theta, \dot{\theta}) \\
\ddot{\theta} & =f_{a e}(\theta, \dot{\theta})=f_{a d}(\theta, \dot{\theta})-f_{s}(\theta, \dot{\theta}),
\end{aligned}
$$

where the function $f_{s}($.$) models the structural properties of the wing apparatus, f_{a d}($. 
models the aerodynamics of the wing oscillations and $f_{a e}($.$) models the aeroelastic$ properties (overall physics). Adding a model noise term to account for the unmodeled physics, a general stochastic quasi-steady model can be written as

$$
\ddot{\theta}=f_{a e}(\theta, \dot{\theta})+\sigma \xi(t)
$$

where $\xi(t)$ is a gaussian white noise with unit strength and $\sigma^{2}$ is the variance of the modelling error.

The measurement equation for the wind-tunnel apparatus under consideration is given as

$$
d_{k}=\theta_{k}+\epsilon_{k}
$$

where $\epsilon_{k} \sim \mathcal{N}\left(0, \gamma^{2}\right)$ is an additive, white Gaussian noise. In this investigation, the variance $\gamma^{2}$ of the measurement noise is assumed to be known from the static response obtained from the wind-tunnel data acquisition system, while the variance $\sigma^{2}$ of the modelling noise is obtained in the parameter estimation step.

\subsection{Numerical validation}

In this section, we validate the proposed model selection and parameter estimation scheme for the following two types of pure pitch oscillations: 1) free decay, and 2) LCO, similar to what is noticed in the wind-tunnel. Note that both the numerical studies are purely statistical in nature and does not have any relation to the problem at hand, except the general model structure of the oscillations and the characteristics of the measurement data. 


\subsubsection{Case 1: Free decay oscillation}

We generate a surrogate free decay response through following model

$$
\ddot{\theta}=e_{1} \operatorname{sign}(\dot{\theta})+e_{2} \theta+e_{3} \dot{\theta}+e_{4} \theta^{3}+e_{5} \xi(t),
$$

While $e_{2} \theta$ and $e_{3} \dot{\theta}$ represent the linear stiffness and linear damping, $\operatorname{sign}(\dot{\theta})$ models the dry friction and $e_{4} \theta^{3}$ models the cubic structural stiffness. The forward integration is performed by substituting following values in Eq. (85): $e_{1}=-0.8, e_{2}=-250.0, e_{3}$ $=-0.8, e_{4}=5000.0$ and $e_{5}=0.05$. The values of $\gamma^{2}$ is substituted as $1 \times 10^{-05}$, similar to the wind tunnel free-decay data. Fig. 17 shows the true response and the measured response. Next, the measured response $\mathbf{d}$ is filtered using a low-pass FIR filter with a cutoff frequency of $30 \mathrm{~Hz}$.
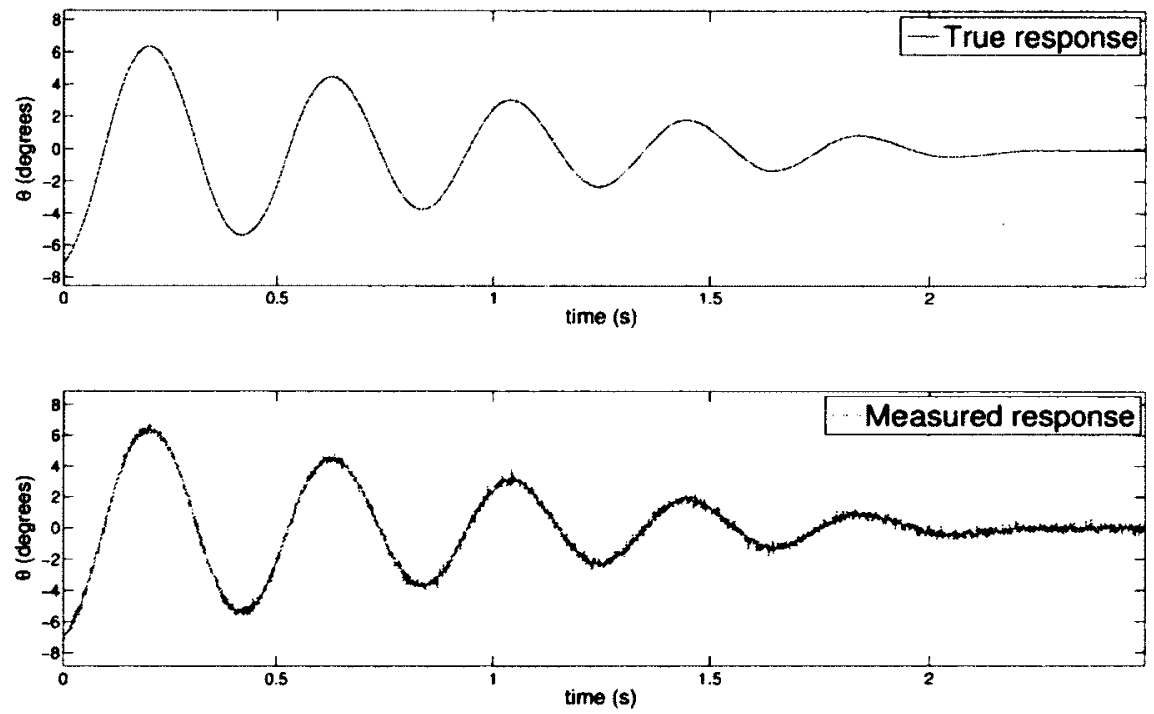

Figure 17: True and measured free decay responses

To start with, following generalized $3^{\text {th }}$ order model is chosen as the initial overly 
complex global system to model the filtered free-decay response:

$$
\begin{aligned}
\ddot{\theta}= & e_{1} \operatorname{sign}(\dot{\theta})+e_{2}+e_{3} \theta+e_{4} \dot{\theta}+e_{5} \theta^{2}+e_{6} \theta \dot{\theta}+e_{7} \dot{\theta}^{2} \\
& +e_{8} \theta^{3}+e_{9} \theta \dot{\theta}^{2}+e_{10} \theta^{2} \dot{\theta}+e_{11} \dot{\theta}^{3}+e_{12} \xi(t)
\end{aligned}
$$

The stepwise term reduction and model selection process described in Chapter 3 is executed to identify the best model nested under the global model in Eq. (86). Tables 2 to 5 shows the model selection data for $\triangle \mathrm{BIC}_{i, j}^{\text {exact }}, \triangle \mathrm{ICOMP}_{i}, \Delta \mathrm{AIC}_{i}$ and $\triangle \mathrm{BIC}_{i}$ respectively.

\begin{tabular}{|c|r|r|r|r|r|r|r|r|}
\hline \multirow{2}{*}{ Term } & \multicolumn{7}{|c|}{$\Delta \mathrm{BIC}_{r, G}^{\text {exact }}$ values } \\
\cline { 2 - 9 } & 1 & 2 & 3 & 4 & 5 & 6 & 7 & 8 \\
\hline$e_{1} \operatorname{sign}(\dot{\theta})$ & 32.0 & 32.9 & 34.0 & 41.1 & 41.4 & 42.1 & 44.3 & 49.2 \\
\hline$e_{2}$ & -4.5 & - & - & - & - & - & - & - \\
\hline$e_{3} \theta$ & 184.5 & 189.9 & 194.4 & 198.1 & 202.0 & 220.6 & 228.1 & 232.1 \\
\hline$e_{4} \dot{\theta}$ & 12.3 & 13.3 & 14.7 & 15.5 & 14.4 & 14.8 & 15.7 & 32.9 \\
\hline$e_{5} \theta^{2}$ & 8.1 & 7.1 & 6.1 & 5.8 & 5.4 & 5.7 & - & - \\
\hline$e_{6} \theta \dot{\theta}$ & 4.1 & 3.5 & 4.3 & 4.5 & - & - & - & - \\
\hline$e_{7} \dot{\theta}^{2}$ & -3.6 & -4.0 & - & - & - & - & - & - \\
\hline$e_{8} \theta^{3}$ & 75.4 & 73.8 & 72.2 & 73.6 & 68.6 & 72.9 & 79.2 & 81.0 \\
\hline$e_{9} \theta^{2} \dot{\theta}$ & 10.1 & 9.6 & 10.2 & 9.7 & 8.9 & 9.0 & 9.0 & - \\
\hline$e_{10} \theta \dot{\theta}^{2}$ & 5.0 & 4.1 & 4.8 & 4.8 & 4.5 & - & - & - \\
\hline$e_{11} \dot{\theta}^{3}$ & -2.6 & -3.3 & -2.5 & - & - & - & - & - \\
\hline
\end{tabular}

Table 2: Free decay case: BIC ${ }^{\text {exact }}$ model selection data

The final model selected by all the four criteria is same as the generating model, i.e.

$$
\ddot{\theta}=e_{1} \operatorname{sign}(\dot{\theta})+e_{2} \theta+e_{3} \dot{\theta}+e_{4} \theta^{3}+e_{5} \xi(t)
$$




\begin{tabular}{|c|r|r|r|r|r|r|r|r|}
\hline \multirow{2}{*}{ Term } & \multicolumn{7}{|c|}{$\Delta$ ICOMP $_{r, G}$ values } \\
\cline { 2 - 9 } & 1 & 2 & 3 & 4 & 5 & 6 & 7 & 8 \\
\hline$e_{1} \operatorname{sign}(\dot{\theta})$ & 34.2 & 47.0 & 55.1 & 54.3 & 52.4 & 51.0 & 50.8 & 53.8 \\
\hline$e_{2}$ & -14.9 & -14.5 & -13.8 & - & - & - & - & - \\
\hline$e_{3} \theta$ & 211.2 & 243.2 & 245.8 & 246.6 & 245.8 & 243.5 & 246.8 & 245.3 \\
\hline$e_{4} \dot{\theta}$ & 8.2 & 17.1 & 18.9 & 19.2 & 18.3 & 16.0 & 15.7 & 35.3 \\
\hline$e_{5} \theta^{2}$ & -3.1 & -2.4 & -1.6 & -1.9 & -4.6 & -4.7 & - & - \\
\hline$e_{6} \theta \dot{\theta}$ & -6.9 & -5.6 & -4.8 & -5.2 & -5.0 & - & - & - \\
\hline$e_{7} \dot{\theta}^{2}$ & -14.2 & -13.2 & -12.4 & -12.4 & - & - & - & - \\
\hline$e_{8} \theta^{3}$ & 21.4 & 51.2 & 56.0 & 59.1 & 60.7 & 61.2 & 69.4 & 41.0 \\
\hline$e_{9} \theta^{2} \dot{\theta}$ & -0.9 & -1.9 & 0.3 & 0.3 & 0.6 & -0.3 & -0.1 & - \\
\hline$e_{10} \dot{\theta}^{2}$ & -21.7 & - & - & - & - & - & - & - \\
\hline$e_{11} \dot{\theta}^{3}$ & -14.0 & -14.8 & - & - & - & - & - & - \\
\hline
\end{tabular}

Table 3: Free decay case: ICOMP model selection data

\begin{tabular}{|c|r|r|r|r|r|r|r|r|}
\hline \multirow{2}{*}{ Term } & \multicolumn{8}{|c|}{$\Delta \mathrm{AIC}_{r, G}$ values } \\
\cline { 2 - 9 } & 1 & 2 & 3 & 4 & 5 & 6 & 7 & 8 \\
\hline$e_{1} \operatorname{sign}(\dot{\theta})$ & 55.9 & 56.0 & 56.6 & 56.3 & 56.2 & 68.3 & 67.2 & 67.0 \\
\hline$e_{2}$ & -1.7 & -1.9 & -1.4 & -1.2 & -1.0 & -0.7 & -1.8 & - \\
\hline$e_{3} \theta$ & 238.7 & 257.9 & 258.5 & 258.3 & 257.5 & 257.1 & 257.4 & 257.3 \\
\hline$e_{4} \dot{\theta}$ & 24.7 & 24.8 & 25.4 & 26.0 & 25.2 & 47.4 & 47.3 & 47.4 \\
\hline$e_{5} \theta^{2}$ & -1.4 & -1.2 & -0.8 & -1.0 & -1.1 & -0.8 & - & - \\
\hline$e_{6} \theta \dot{\theta}$ & -1.5 & -1.7 & -1.3 & -1.2 & - & - & - & - \\
\hline$e_{7} \dot{\theta}^{2}$ & -2.0 & -2.2 & -1.8 & - & - & - & - & - \\
\hline$e_{8} \theta^{3}$ & 68.1 & 81.7 & 82.0 & 81.8 & 81.1 & 80.4 & 83.1 & 83.7 \\
\hline$e_{9} \theta^{2} \dot{\theta}$ & -2.2 & -2.4 & - & - & - & - & - & - \\
\hline$e_{10} \dot{\theta}^{2}$ & -2.3 & - & - & - & - & - & - & - \\
\hline$e_{11} \dot{\theta}^{3}$ & -2.1 & -2.3 & -1.1 & -0.7 & -1.3 & - & - & - \\
\hline
\end{tabular}

Table 4: Free decay case: AIC model selection data 


\begin{tabular}{|c|c|c|c|c|c|c|c|c|}
\hline \multirow{2}{*}{ Term } & \multicolumn{8}{|c|}{$\Delta \mathrm{BIC}_{r, G}$ values } \\
\hline & 1 & 2 & 3 & 4 & 5 & 6 & 7 & 8 \\
\hline$e_{1} \operatorname{sign}(\dot{\theta})$ & 50.0 & 50.2 & 50.8 & 50.5 & 50.4 & 62.5 & 61.4 & 61.2 \\
\hline$e_{2}$ & -7.5 & -7.7 & -7.2 & -7.0 & -6.9 & -6.5 & -7.7 & - \\
\hline$e_{3} \theta$ & 232.9 & 252.0 & 252.7 & 252.5 & 251.7 & 251.2 & 251.5 & 251.5 \\
\hline$e_{4} \dot{\theta}$ & 18.9 & 19.0 & 19.5 & 20.2 & 19.3 & 41.6 & 41.4 & 41.5 \\
\hline$e_{5} \theta^{2}$ & -7.2 & -7.1 & -6.6 & -6.8 & -7.0 & -6.6 & - & - \\
\hline$e_{6} \theta \dot{\theta}$ & -7.3 & -7.5 & -7.1 & -7.1 & - & - & - & - \\
\hline$e_{7} \dot{\theta}^{2}$ & -7.9 & -8.0 & -7.6 & - & - & - & - & - \\
\hline$e_{8} \theta^{3}$ & 62.2 & 75.8 & 76.2 & 76.0 & 75.3 & 74.6 & 77.3 & 77.9 \\
\hline$e_{9} \theta^{2} \dot{\theta}$ & -8.0 & -8.2 & - & - & - & - & - & - \\
\hline$e_{10} \theta \dot{\theta}^{2}$ & -8.1 & - & - & - & - & - & - & - \\
\hline$e_{11} \dot{\theta}^{3}$ & -7.9 & -8.1 & -6.9 & -6.5 & -7.1 & 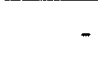 & - & - \\
\hline
\end{tabular}

Table 5: Free decay case: BIC model selection data

\subsubsection{Case 2 : Limit cycle oscillations}

A surrogate LCO response, similar to the one in Fig. 15, is generated using the following generating model:

$$
\ddot{\theta}=e_{1} \operatorname{sign}(\dot{\theta})+e_{2} \theta+e_{3} \dot{\theta}+e_{4} \theta^{2}+e_{5} \theta^{3}+e_{6} \theta^{2} \dot{\theta}+e_{7} \xi(t)
$$

The following parameter values are substituted: $e_{1}=0.001, e_{2}=-540.0, e_{3}=4.0, e_{4}$ $=-180.0, e_{5}=24000.0, e_{6}=-2200.0$ and $e_{7}=0.07$. The values of $\gamma^{2}$ is substituted as $5 \times 10^{-06}$, similar to the wind tunnel LCO data. Fig. 18 shows the true response and the measured response. Next, the measured response $\mathbf{d}$ is filtered using a low-pass FIR filter with a cutoff frequency of $30 \mathrm{~Hz}$.

To start with, the following generalized $3^{\text {th }}$ order model is chosen as the initial 

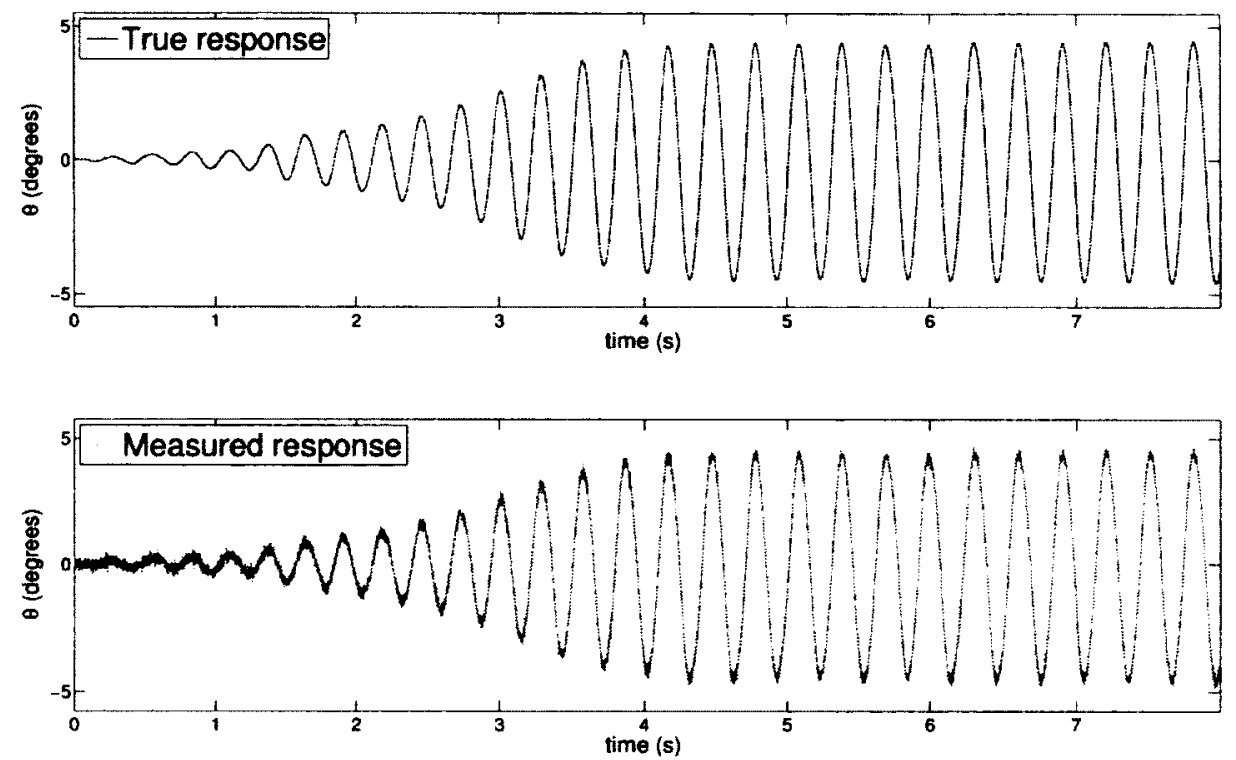

Figure 18: True and measured LCO response

overly complex global system to model the filtered LCO response:

$$
\begin{aligned}
\ddot{\theta}= & e_{1} \operatorname{sign}(\dot{\theta})+e_{2}+e_{3} \theta+e_{4} \dot{\theta}+e_{5} \theta^{2}+e_{6} \theta \dot{\theta}+e_{7} \dot{\theta}^{2} \\
& +e_{8} \theta^{3}+e_{9} \theta^{2} \dot{\theta}+e_{10} \theta \dot{\theta}^{2}+e_{11} \dot{\theta}^{3}+e_{12} \xi(t) .
\end{aligned}
$$

The stepwise term reduction and model selection process described in Chapter 3 is executed to identify the best model nested under the global model in Eq. (89). Tables 6 to 9 shows the model selection data for BIC ${ }^{\text {exact }}$, ICOMP, AIC and BIC respectively.

The final model selected by $\Delta \mathrm{BIC}_{r, G}^{\text {exact }}, \Delta \mathrm{BIC}_{r, G}$ and $\Delta \mathrm{ICOMP}_{r, G}$ is

$$
\ddot{\theta}=e_{3} \theta+e_{4} \dot{\theta}+e_{5} \theta^{2}+e_{8} \theta^{3}+e_{9} \theta^{2} \dot{\theta}+e_{11} \dot{\theta}^{3}+e_{12} \xi(t)
$$

This model differs from the generating model in Eq. (88) in one term; the dry friction term is replaced by the $\dot{\theta}^{3}$ term. On the other hand, the final model selected by 


\begin{tabular}{|c|r|r|r|r|r|r|}
\hline \multirow{2}{*}{ Term } & \multicolumn{7}{|c|}{$\Delta \mathrm{BIC}_{r, G}^{\text {exact }}$ values } \\
\cline { 2 - 7 } & 1 & 2 & 3 & 4 & 5 & 6 \\
\hline$e_{1} \operatorname{sign}(\dot{\theta})$ & 2.41 & 2.34 & 2.17 & 2.25 & - & - \\
\hline$e_{2}$ & -4.17 & -4.70 & - & - & - & - \\
\hline$e_{3} \theta$ & 635.89 & 640.38 & 644.76 & 648.75 & 663.09 & 834.23 \\
\hline$e_{4} \dot{\theta}$ & 36.62 & 36.69 & 36.75 & 37.11 & 87.20 & 90.71 \\
\hline$e_{5} \theta^{2}$ & 70.06 & 70.55 & 110.01 & 110.77 & 108.83 & 108.40 \\
\hline$e_{6} \theta \dot{\theta}$ & 1.65 & 1.61 & 1.59 & - & - & - \\
\hline$e_{7} \dot{\theta}^{2}$ & -5.21 & - & - & - & - & - \\
\hline$e_{8} \theta^{3}$ & 254.85 & 256.92 & 259.12 & 260.94 & 264.92 & 311.88 \\
\hline$e_{9} \theta^{2} \dot{\theta}$ & 364.97 & 367.67 & 369.97 & 372.60 & 377.70 & 386.41 \\
\hline$e_{10} \theta \dot{\theta}^{2}$ & 4.47 & 4.31 & 4.31 & 4.36 & 4.90 & - \\
\hline$e_{11} \dot{\theta}^{3}$ & 4.08 & 3.96 & 4.03 & 3.96 & 44.04 & 42.38 \\
\hline
\end{tabular}

Table 6: $\mathrm{LCO}$ case: $\mathrm{BIC}^{\text {exact }}$ model selection data

\begin{tabular}{|c|r|r|r|r|r|r|}
\hline \multirow{2}{*}{ Term } & \multicolumn{7}{|c|}{$\Delta \mathrm{ICOMP}_{r, G}$ values } \\
\cline { 2 - 7 } & 1 & 2 & 3 & 4 & 5 & 6 \\
\hline$e_{1} \operatorname{sign}(\dot{\theta})$ & -7.90 & -8.30 & -8.15 & -8.43 & - & - \\
\hline$e_{2}$ & -14.73 & -15.40 & - & - & - & - \\
\hline$e_{3} \theta$ & 651.56 & 653.05 & 655.27 & 656.82 & 668.49 & 840.41 \\
\hline$e_{4} \dot{\theta}$ & 29.32 & 29.16 & 28.77 & 28.64 & 82.87 & 84.99 \\
\hline$e_{5} \theta^{2}$ & 65.90 & 65.48 & 108.72 & 108.30 & 105.03 & 103.05 \\
\hline$e_{6} \theta \dot{\theta}$ & -8.94 & -9.26 & -9.14 & - & - & - \\
\hline$e_{7} \dot{\theta}^{2}$ & -15.67 & - & - & - & - & - \\
\hline$e_{8} \theta^{3}$ & 211.01 & 215.87 & 220.33 & 225.12 & 231.84 & 282.93 \\
\hline$e_{9} \theta^{2} \dot{\theta}$ & 385.22 & 384.70 & 384.40 & 383.96 & 387.19 & 390.52 \\
\hline$e_{10} \theta \dot{\theta}^{2}$ & -6.37 & -6.61 & -6.52 & -6.53 & -5.77 & - \\
\hline$e_{11} \dot{\theta}^{3}$ & -5.64 & -5.53 & -5.93 & -6.11 & 36.75 & 34.34 \\
\hline
\end{tabular}

Table 7: LCO case: ICOMP model selection data 


\begin{tabular}{|c|r|r|r|r|r|}
\hline \multirow{2}{*}{ Term } & \multicolumn{5}{|c|}{$\Delta \mathrm{AIC}_{r, G}$ values } \\
\cline { 2 - 6 } & 1 & 2 & 3 & 4 & 5 \\
\hline$e_{1} \operatorname{sign}(\dot{\theta})$ & 3.66 & 3.53 & 3.43 & 3.31 & 3.99 \\
\hline$e_{2}$ & -1.63 & -1.62 & -1.73 & - & - \\
\hline$e_{3} \theta$ & 675.56 & 675.36 & 675.24 & 675.03 & 851.53 \\
\hline$e_{4} \dot{\theta}$ & 39.33 & 39.21 & 39.14 & 38.89 & 41.38 \\
\hline$e_{5} \theta^{2}$ & 67.20 & 67.03 & 67.06 & 110.39 & 109.63 \\
\hline$e_{6} \theta \dot{\theta}$ & -2.00 & - & - & - & - \\
\hline$e_{7} \dot{\theta}^{2}$ & -1.93 & -1.90 & - & - & - \\
\hline$e_{8} \theta^{3}$ & 261.28 & 261.13 & 260.99 & 260.93 & 302.51 \\
\hline$e_{9} \theta^{2} \dot{\theta}$ & 382.43 & 382.34 & 382.41 & 382.05 & 389.29 \\
\hline$e_{10} \theta \dot{\theta}^{2}$ & -0.72 & -0.72 & -0.77 & -0.67 & - \\
\hline$e_{11} \dot{\theta}^{3}$ & 6.17 & 6.19 & 6.03 & 5.95 & 4.98 \\
\hline
\end{tabular}

Table 8: LCO case: AIC model selection data

\begin{tabular}{|c|r|r|r|r|r|r|}
\hline \multirow{2}{*}{ Term } & \multicolumn{7}{|c|}{$\Delta \mathrm{BIC}_{r, G}$ values } \\
\cline { 2 - 7 } & 1 & 2 & 3 & 4 & 5 & 6 \\
\hline$e_{1} \operatorname{sign}(\dot{\theta})$ & -3.03 & -3.18 & 3.43 & -3.39 & -2.71 & - \\
\hline$e_{2}$ & -8.33 & -8.33 & -1.73 & - & - & - \\
\hline$e_{3} \theta$ & 668.87 & 668.65 & 675.24 & 668.33 & 844.83 & 852.04 \\
\hline$e_{4} \dot{\theta}$ & 32.64 & 32.50 & 39.14 & 32.18 & 34.68 & 90.17 \\
\hline$e_{5} \theta^{2}$ & 60.51 & 60.32 & 67.06 & 103.69 & 102.93 & 99.43 \\
\hline$e_{6} \theta \dot{\theta}$ & -8.69 & - & - & - & - & - \\
\hline$e_{7} \dot{\theta}^{2}$ & -8.62 & -8.61 & - & - & - & - \\
\hline$e_{8} \theta^{3}$ & 254.59 & 254.43 & 260.99 & 254.22 & 295.81 & 303.50 \\
\hline$e_{9} \theta^{2} \dot{\theta}$ & 375.74 & 375.63 & 382.41 & 375.35 & 382.60 & 385.51 \\
\hline$e_{10} \theta \dot{\theta}^{2}$ & -7.42 & -7.42 & -0.77 & -7.38 & - & - \\
\hline$e_{11} \dot{\theta}^{3}$ & -0.53 & -0.51 & 6.03 & -0.75 & -1.72 & 41.18 \\
\hline
\end{tabular}

Table 9: LCO case: BIC model selection data 
$\Delta \mathrm{AIC}_{r, G}$ is

$$
\ddot{\theta}=e_{1} \operatorname{sign}(\dot{\theta})+e_{3} \theta+e_{4} \dot{\theta}+e_{5} \theta^{2}+e_{8} \theta^{3}+e_{9} \theta^{2} \dot{\theta}+e_{11} \dot{\theta}^{3}+e_{12} \xi(t)
$$

This model has an additional term of $\dot{\theta}^{3}$ compared to the generating model.

\subsubsection{Important observations}

The following observations are made using the model selection and parameter estimation results of the two numerical studies:

- The agreement between the BIC and BIC ${ }^{\text {exact }}$ criteria for both the studies indicates that the filtered response used for the model selection has a higher possibility of being in the "Large data set" regime, an assumption for BIC to approximate $\mathrm{BIC}^{\text {exact }}[1,12]$. Since we have only considered a single realization of the measured response, we cannot be sure about this possibility. Therefore, when in doubt, it is always safe to use the non-asymptotic criteria of BIC ${ }^{\text {exact }}$. The agreement between $\mathrm{BIC}$ and $\mathrm{BIC}^{\text {exact }}$ also provides an assurance of the goodness of the Chib-Jeliazkov method in estimating $\Delta \mathrm{BIC}_{r, G}^{\text {exact }}$ and the insensitivity of the flat priors in performing model selection through a stepwise term reduction procedure based on the Bayes factor criterion.

- For the free decay case, the posterior marginal pdfs of parameter $e_{1}, e_{3}, e_{4}$ and $e_{8}$ for the final chosen model are contrasted with those from the global model in Fig. 19. The pdfs are normalized histograms generated using 3.2 million MCMC samples obtained from 32 parallel MCMC chains. The pdfs in the case of the final model are more peaked than the ones from the global model. This behaviour is a testament of the bias-variance trade-off principle being achieved 
by the model selection procedures. Two predictive models were obtained by replacing the static parameters in the global model in Eq. (86) and the final model in Eq. (85) with the corresponding joint posterior distributions obtained using Bayesian inference. A random sample is drawn from the posterior pdf to generate a response realization. Fig. 20 shows the comparison of the 100 response realizations from the two models and the filtered response used for modelling. Notice the large variation in the response for the global predictive model when compared to the final predictive model. This observation further iterates the necessity of the model selection procedures and their application of the bias-variation trade-off. Similar arguments can be made from the results of the LCO case.
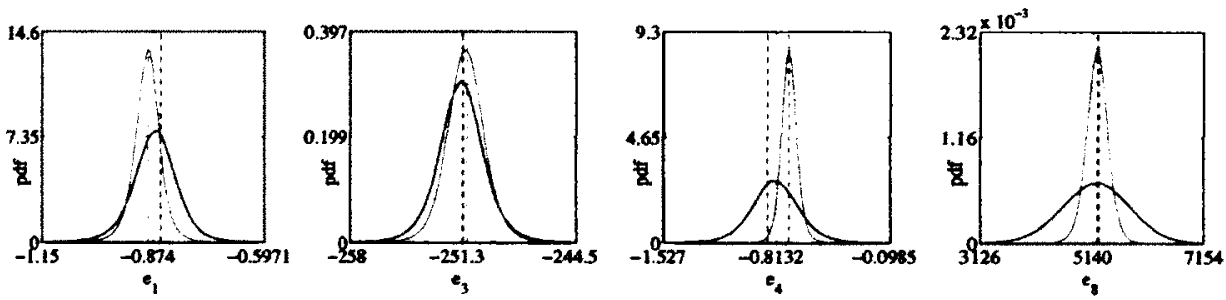

Figure 19: Comparison of posterior marginal pdfs in the final model (red) and the global model (blue)

- In the LCO case, the disagreement between the generating model and the final chosen model by the $\mathrm{BIC}^{\text {exact }}$ criteria reiterates the fact that the final model chosen may not always be the best model nested under the initial global model. This is a well known short coming of the stepwise model selection procedures $[64-66]$. However, the purpose of the model selection methods is not to uncover the generating model, but to find a model which is simple enough to be used for predictions and complex enough to represent the nonlinear physics. Since the chosen model is comparable to the generating model in its complexity and capability to represent the physics, the objective of the model selection 

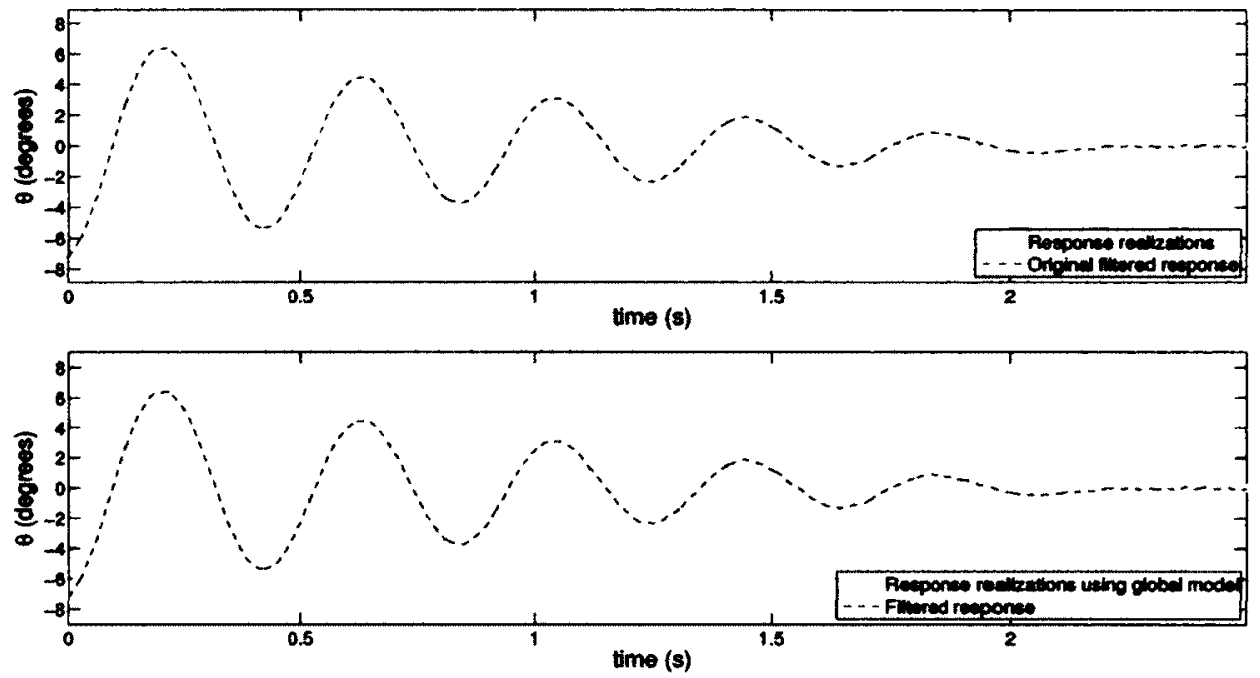

Figure 20: Comparison of response realizations (pink) with the filtered response (blue)

exercise is successfully fulfilled.

- The selection of an overly complex model for the LCO case by $\triangle \mathrm{AIC}_{r, G}$ is a well known shortcoming of the AIC criteria. However, this statement is incomplete without the consideration of the model selection uncertainty, which is a highly debatable issue in the literature $[1,45,95]$. However, as proved by Konishi and Kitagawa [1], the model selection uncertainty is fairly low when large data is used in modelling. We will however refrain from using any asymptotic method and will use the $\mathrm{BIC}^{\text {exact }}$ criteria for modelling the wind-tunnel data.

- The state estimation is successfully performed using EKF. Fig. 21 and Fig. 22 shows the initial stretch of state estimation results for the final chosen model in the free decay and the LCO case respectively.

- Based on the model selection data of the $\triangle \mathrm{BIC}_{r, G}^{\text {exact }}$ shown in Table 2 and Table 6 , the table for the $\Delta \mathrm{BIC}_{r, G}^{\text {exact }}$ benchmark values provided by Kass [12] is updated under the current model selection context as follows: 


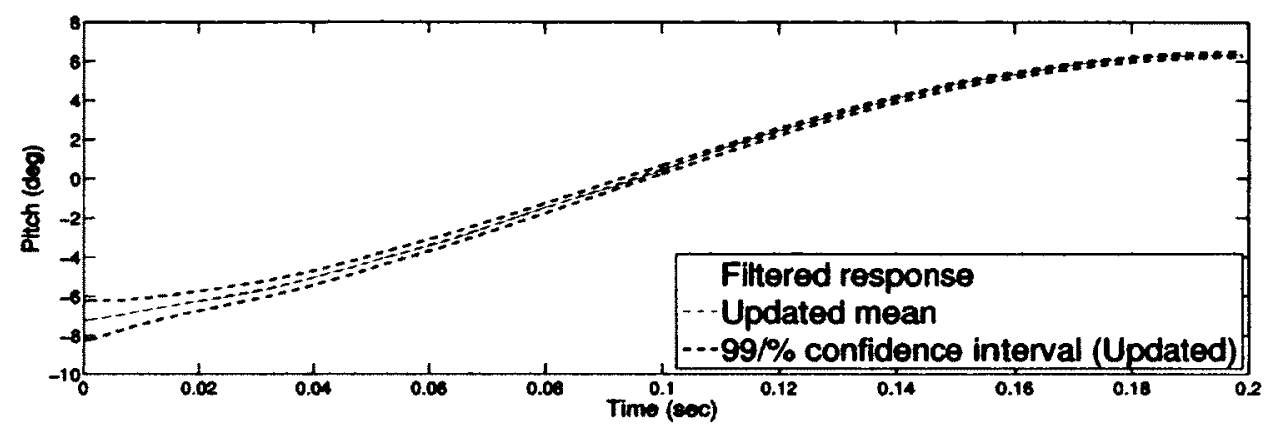

Figure 21: Free decay case: State estimation results for the final model when $\phi=\hat{\phi}$

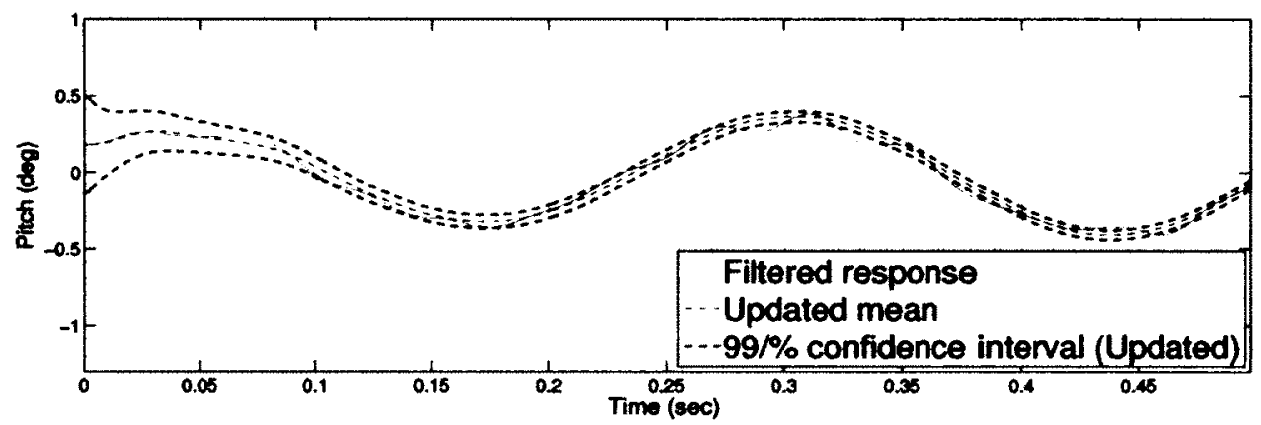

Figure 22: LCO case: State estimation results for the final model when $\phi=\hat{\phi}$

\begin{tabular}{|c|l|}
\hline$\Delta \mathrm{BIC}_{r, G}^{\text {exact }}$ & Evidence against $\mathcal{M}_{r}$ \\
\hline$<10$ & Bare minimum \\
10 to 25 & Positive \\
$>25$ & Very strong \\
\hline
\end{tabular}

Table 10: Updated $\triangle \mathrm{BIC}_{r, G}^{\text {exact }}$ benchmark values for model selection when 
Next, we model the wind-tunnel data using the benchmark values of $\Delta \mathrm{BIC}_{r, G}^{\text {exact }}$ derived in Table 10.

\subsection{Modelling of the wind-tunnel data}

\subsubsection{Free-decay response with no airflow}

A pure rotation free decay response of the NACA0012 wing under no air-flow conditions was captured for modelling the structural properties of the wing in the pitch dof. The structural parameters will eventually help in identifying the aerodynamic contributions to the LCO aerodynamics. Fig. 23 shows the measured response and its filtered version.
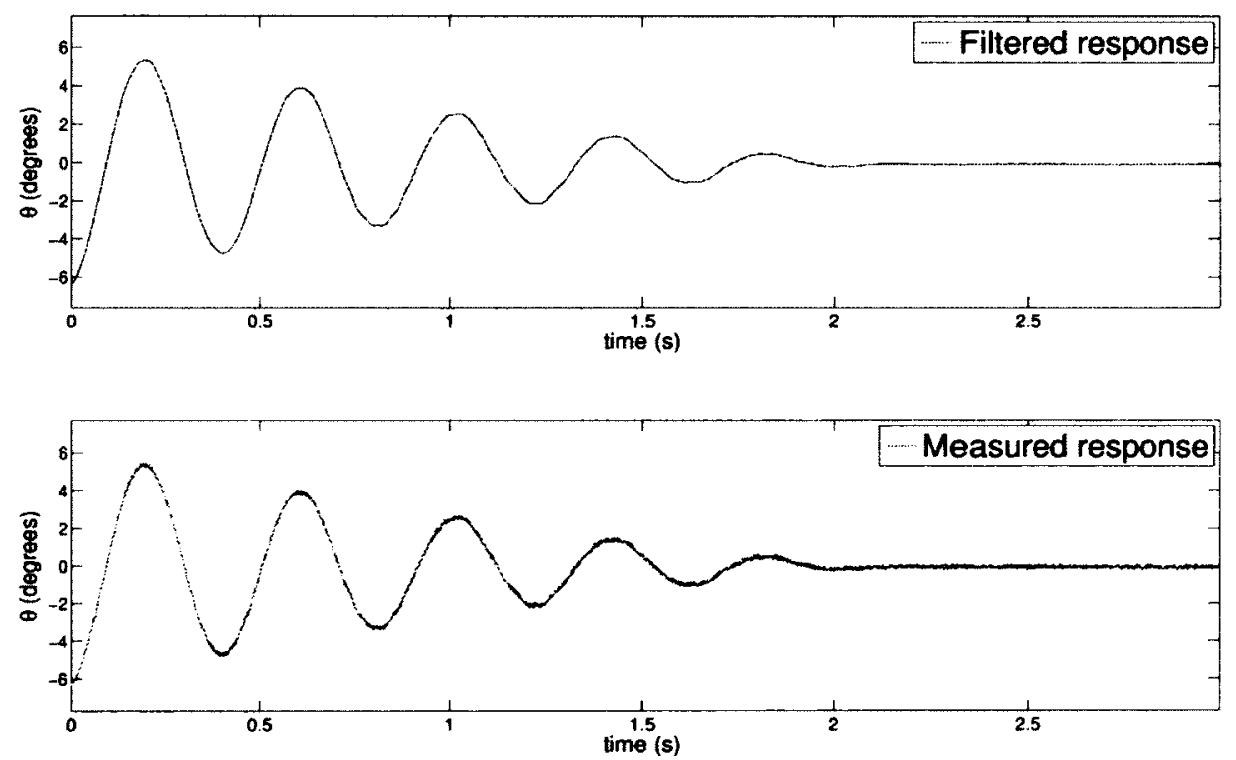

Figure 23: Free decay response with no airflow 
We propose the following global model to start the model selection process:

$$
\begin{aligned}
\ddot{\theta}= & c_{1} \operatorname{sign}(\dot{\theta})+c_{2}+c_{3} \theta+c_{4} \dot{\theta}+c_{5} \theta^{2}+c_{6} \theta \dot{\theta}+c_{7} \dot{\theta}^{2} \\
& +c_{8} \theta^{3}+c_{9} \theta \dot{\theta}^{2}+c_{10} \theta^{2} \dot{\theta}+c_{11} \dot{\theta}^{3}+c_{12} \xi(t) .
\end{aligned}
$$

The "sign" term is added to account for the dry friction that may be present in the wing apparatus. The parameter $c_{2}$ accounts for the bias that may be introduced by the data collecting apparatus. The rest of the right-hand side in Eq. (92) models the stiffness and damping of the wing apparatus. Our aim here is to uncover an optimal model nested under the global model in Eq. (92) through a stepwise term reduction using the $\mathrm{BIC}^{\text {exact }}$ criterion. Since there is no airflow, there are no aerodynamic contributions to the physics or $f_{a e}=f_{s}$ with $f_{a d}=0$ in Eq. (82b).

Table 11 shows the model selection data for the filtered free decay response. The final model selected is

$$
\ddot{\theta}=c_{1} \operatorname{sign}(\dot{\theta})+c_{2}+c_{3} \theta+c_{4} \dot{\theta}+c_{8} \theta^{3}+c_{12} \xi(t)
$$

Using the parameter estimation strategy discussed in Chapter $4,3.2 \times 10^{6} \mathrm{MCMC}$ samples were obtained from the joint posterior pdf of the static parameters of the chosen model using 32 parallel chains. The marginal pdfs (in contrast to the MLE estimates) and the 2-dimensional joint pdfs with a correlation coefficient greater than 0.5 are shown in Fig. 24 and Fig. 25 respectively. The MLE estimates are as follows: $c_{1}=-0.8164, c_{2}=-0.3100, c_{3}=-242.0520, c_{4}=-0.7928$ and $c_{8}=2166.0790$. These MLE estimates will be used for differentiating the aerodynamic contributions from the overall physics of the LCO response in the next section.

In order to understand the effect of model reduction on parameter estimates, we compare the marginal pdfs for parameter $c_{1}, c_{2}, c_{3}, c_{4}, c_{8}$ and $c_{12}$ in the final model in 


\begin{tabular}{|c|r|r|r|r|r|r|r|}
\hline \multirow{2}{*}{ Term } & \multicolumn{7}{|c|}{$\Delta \mathrm{BIC}_{\tau, G}^{\text {exact }}$ values } \\
\cline { 2 - 8 } & 1 & 2 & 3 & 4 & 5 & 6 & 7 \\
\hline$c_{1} \operatorname{sign}(\dot{\theta})$ & 23.1 & 23.6 & 29.4 & 30.0 & 31.2 & 32.0 & 37.4 \\
\hline$c_{2}$ & 10.0 & 14.1 & 14.0 & 14.4 & 14.6 & 26.7 & 27.1 \\
\hline$c_{3} \theta$ & 246.4 & 249.9 & 253.1 & 260.0 & 287.3 & 292.1 & 294.5 \\
\hline$c_{4} \dot{\theta}$ & 7.3 & 6.5 & 18.3 & 19.1 & 18.2 & 18.3 & 32.4 \\
\hline$c_{5} \theta^{2}$ & 7.7 & 6.7 & 7.2 & 7.2 & 7.2 & - & - \\
\hline$c_{6} \theta \dot{\theta}$ & 3.8 & 3.0 & 3.5 & - & - & - & - \\
\hline$c_{7} \dot{\theta}^{2}$ & -3.1 & - & - & - & - & - & - \\
\hline$c_{8} \theta^{3}$ & 33.4 & 33.9 & 33.9 & 33.2 & 31.4 & 32.3 & 32.6 \\
\hline$c_{9} \theta^{2} \dot{\theta}$ & 11.3 & 11.0 & 10.5 & 10.7 & 10.9 & 10.8 & - \\
\hline$c_{10} \dot{\theta}^{2}$ & 8.1 & 7.1 & 7.2 & 6.7 & - & - & - \\
\hline$c_{11} \dot{\theta}^{3}$ & -2.2 & -2.3 & - & - & - & - & - \\
\hline
\end{tabular}

Table 11: Free decay wind-tunnel data: BIC $^{\text {exact }}$ model selection data
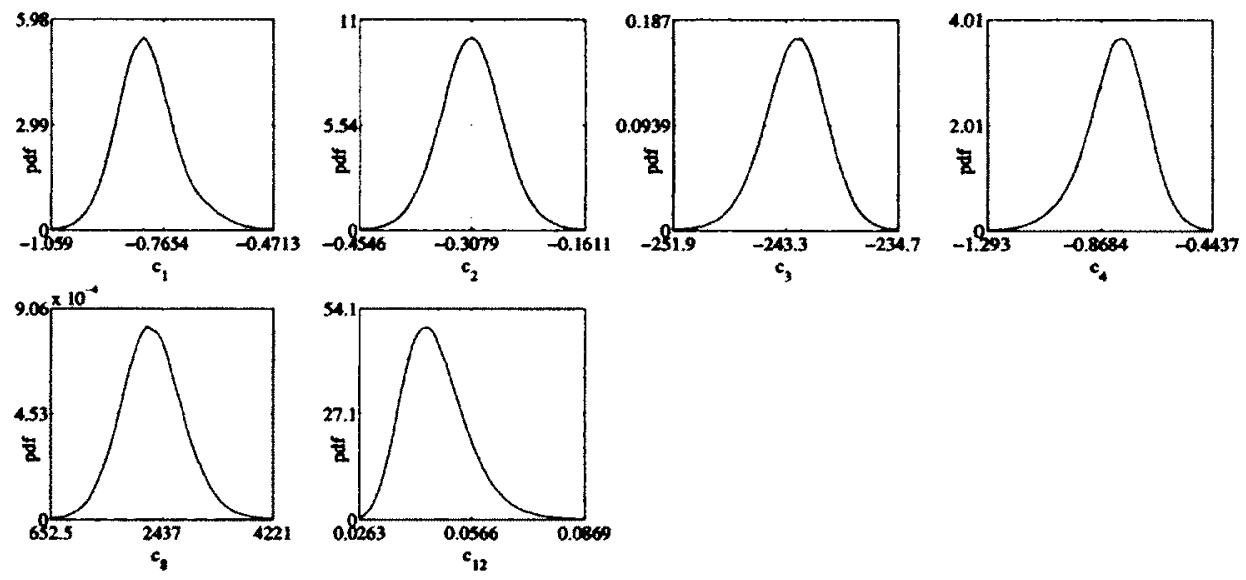

Figure 24: Marginal posterior pdfs and MLE estimate (red-dashed line) for the parameters of the final model
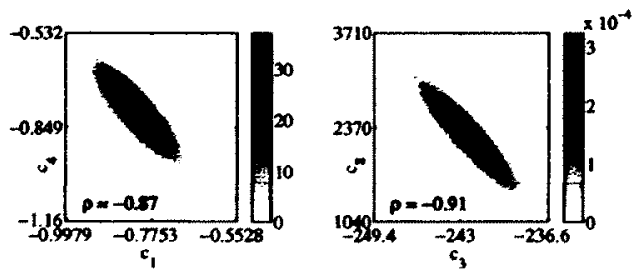

Figure 25: Selected joint posterior pdfs for the parameters of the final model 
Eq. (93) and the initial global model in Eq. (92). The comparison is shown in Fig. 26. The variability in the parameter estimates decreases when fewer terms are present in the model. This observation is in agreement with the findings of the numerical study.
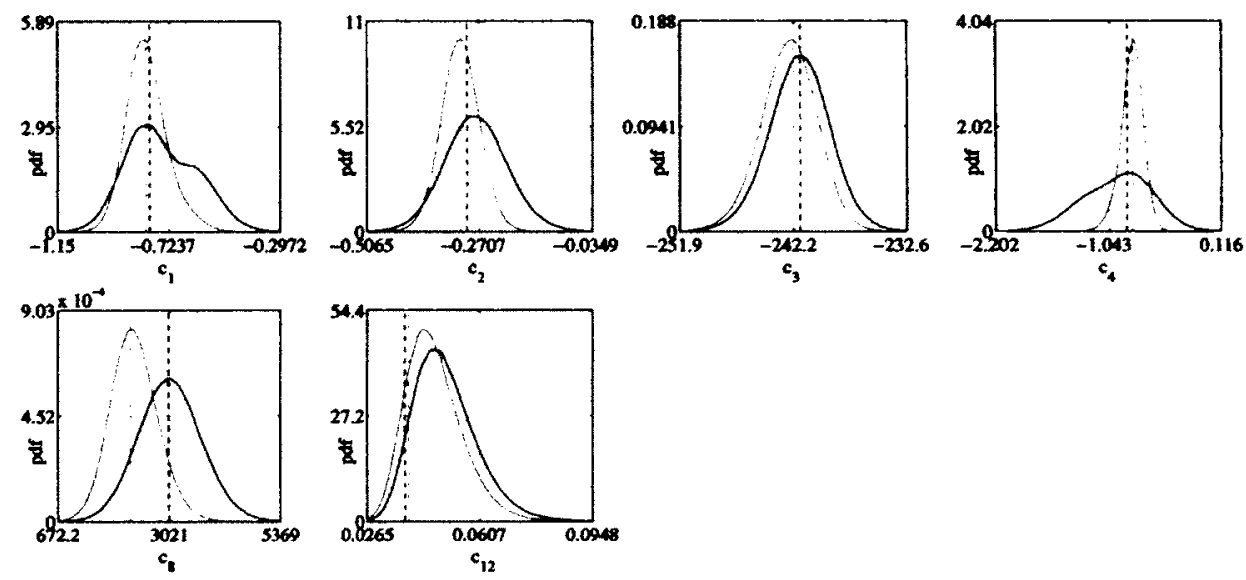

Figure 26: Comparison of marginal pdfs of selected parameters in the final model (red) and the global model (blue)

\subsubsection{Laminar separation flutter}

A pure pitch LCO response for the laminar separation flutter was measured in the $\mathrm{RMC}$ wind-tunnel for an airspeed of $7.5 \mathrm{~m} / \mathrm{s}\left(\mathrm{Re}_{c}=75,000\right), 8.5 \mathrm{~m} / \mathrm{s}\left(\operatorname{Re}_{c}=85,000\right)$, $9.0 \mathrm{~m} / \mathrm{s}\left(\operatorname{Re}_{c}=90,000\right)$ and $10.5 \mathrm{~m} / \mathrm{s}\left(\operatorname{Re}_{c}=105,000\right)$. The measured response and its spectral content is shown in Fig. 15 and Fig. 27 respectively. The presence of the super-harmonics in the spectral content of the oscillations in Fig. 27 further exposes the nonlinear nature of the oscillations.

Next, we filter the measured response using a low-pass FIR filter with the cut-off frequency $f_{c}=25 \mathrm{~Hz}$ for all four Re cases. The value of $f_{c}$ is chosen such that the spectral contributions of $7 f$ harmonics remain unaltered in all the LCO responses; $f$ being the fundamental frequency of the LCO. This $f_{c}$ value also ensures consistent comparison of model selection results over varying Re. Fig. 28 and Fig. 29 shows the 
filtered (trimmed) response and its spectral content. Notice that filtering removes all the nonphysical spectral contributions from the response, including the $30 \mathrm{~Hz}$ noise originating due to the tunnel motor [3]. Fig. 30 shows the comparison a section of the measured and filtered response for $\operatorname{Re}=85,000$.
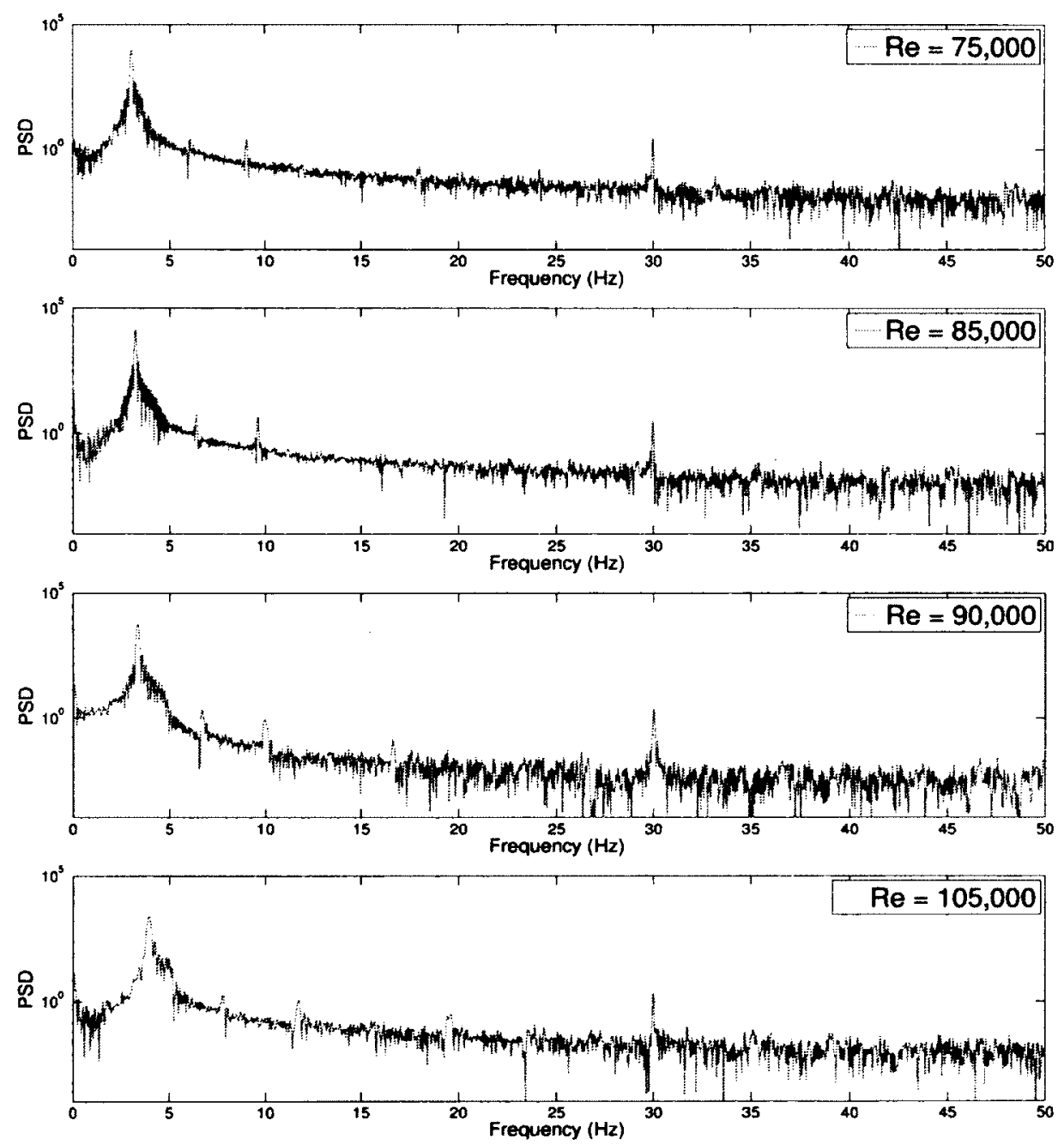

Figure 27: Power spectral density (PSD) of the measured oscillator response

The trimming of the captured response is carried out to remove the undesirable portions of the response from the viewpoint of modelling, mainly the low amplitude oscillation of less than $1^{\circ}$ where the difference between the aerodynamics and the 

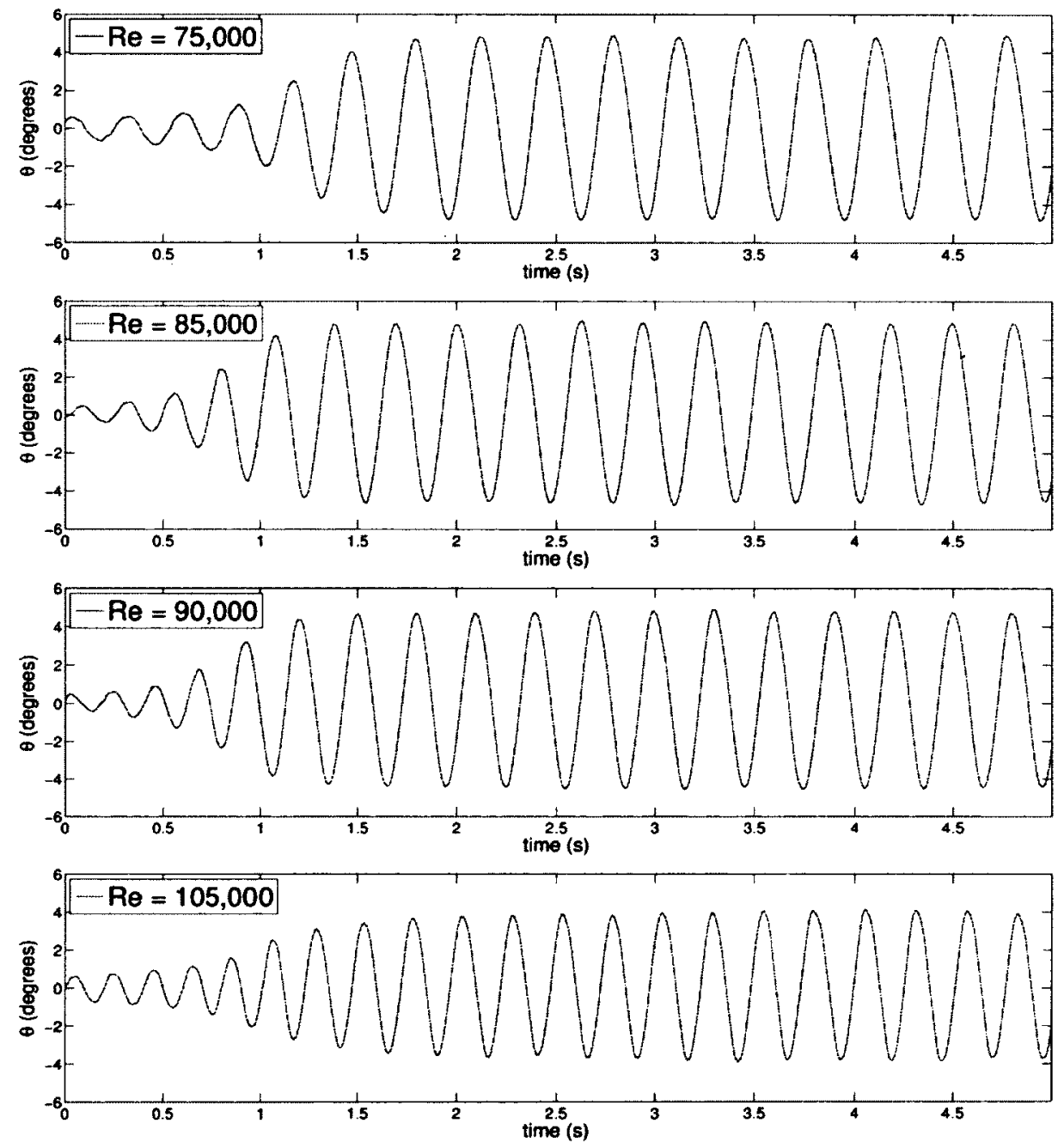

Figure 28: The filtered oscillator response used for system identification 

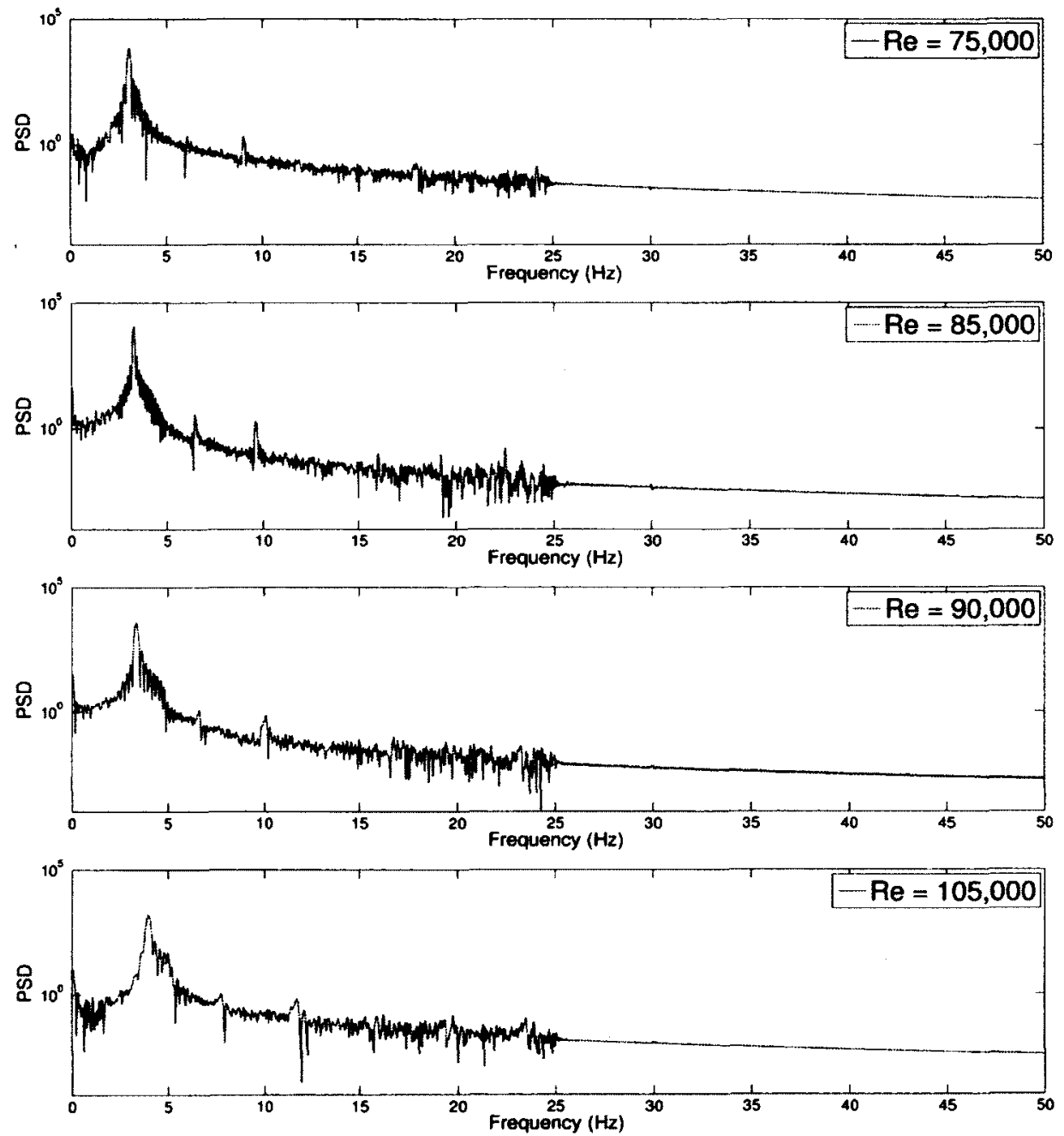

Figure 29: Power spectral density (PSD) of the filtered oscillator response 
turbulence is negligible. Only parts that hold significant information about the aerodynamics of the self-sustained oscillations are considered for the system identification purposes.

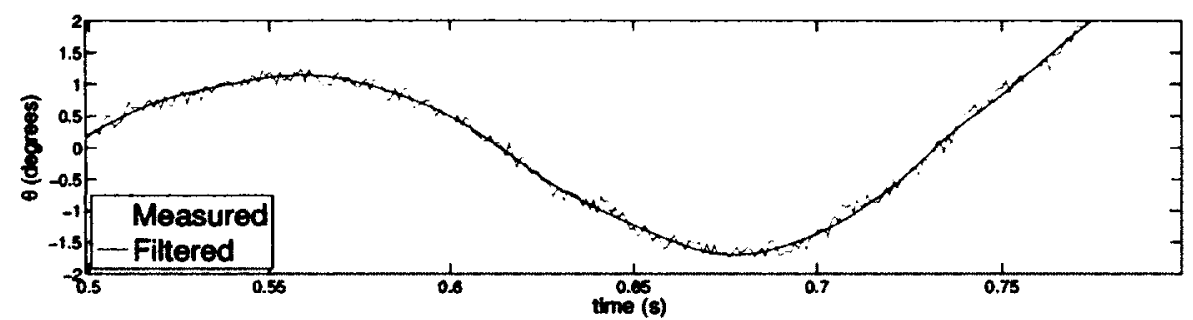

Figure 30: Comparison of the measured and filtered LCO response

Assuming quasi-steady aerodynamics, a generalized $5^{\text {th }}$ order model is proposed as the initial global model for all the four Re responses:

$$
\begin{aligned}
\ddot{\theta}= & c_{1} \operatorname{sign}(\dot{\theta})+e_{1}+e_{2} \theta+e_{3} \dot{\theta}+e_{4} \theta^{2}+e_{5} \theta \dot{\theta}+e_{6} \dot{\theta}^{2} \\
& +e_{7} \theta^{3}+e_{8} \theta \dot{\theta}^{2}+e_{9} \theta^{2} \dot{\theta}+e_{10} \dot{\theta}^{3}+e_{11} \theta^{4}+e_{12} \theta^{3} \dot{\theta} \\
& +e_{13} \theta^{2} \dot{\theta}^{2}+e_{14} \theta \dot{\theta}^{3}+e_{15} \dot{\theta}^{4}+e_{16} \theta^{5}+e_{17} \theta^{4} \dot{\theta}+ \\
& +e_{18} \theta^{3} \dot{\theta}^{2}+e_{19} \theta^{2} \dot{\theta}^{3}+e_{20} \dot{\theta}^{4}+e_{21} \dot{\theta}^{5}+e_{22} \xi(t),
\end{aligned}
$$

Remember that the dry friction coefficient $c_{1}=-0.8164$ is known from the MLE estimates of the final free decay model in Eq. (93).

Tables 12 to 15 shows the BIC ${ }^{\text {exact }}$ model selection data for the four Re values. The terms $\theta, \dot{\theta}$ and $\theta^{3}$ were not considered for the elimination in the stepwise model selection procedure since they had a nonzero structural component to them. The final model selected for each Re value is as follows:

$$
\begin{aligned}
7.5 \mathrm{~m} / \mathrm{s}(9 \text { terms }): \quad \ddot{\theta}= & c_{1} \operatorname{sign}(\dot{\theta})+e_{2} \theta+e_{3} \dot{\theta}+e_{7} \theta^{3}+e_{8} \theta \dot{\theta}^{2} \\
& +e_{9} \theta^{2} \dot{\theta}+e_{16} \theta^{5}+e_{17} \theta^{4} \dot{\theta}+e_{19} \theta^{2} \dot{\theta}^{3}+e_{22} \xi(t)
\end{aligned}
$$


$8.5 \mathrm{~m} / \mathrm{s}(10$ terms $): \quad \ddot{\theta}=c_{1} \operatorname{sign}(\dot{\theta})+e_{2} \theta+e_{3} \dot{\theta}+e_{7} \theta^{3}+e_{9} \theta^{2} \dot{\theta}$

$$
+e_{11} \theta^{4}+e_{16} \theta^{5}+e_{17} \theta^{4} \dot{\theta}+e_{18} \theta^{3} \dot{\theta}^{2}+e_{19} \theta^{2} \dot{\theta}^{3}+e_{22} \xi(t) .
$$

$9.0 \mathrm{~m} / \mathrm{s}(11$ terms $): \quad \ddot{\theta}=c_{1} \operatorname{sign}(\dot{\theta})+e_{2} \theta+e_{3} \dot{\theta}+e_{4} \theta^{2}+e_{7} \theta^{3}$

$$
\begin{aligned}
& +e_{11} \theta^{4}+e_{16} \theta^{5}+e_{17} \theta^{4} \dot{\theta}+e_{18} \theta^{3} \dot{\theta}^{2}+e_{19} \theta^{2} \dot{\theta}^{3} \\
& +e_{20} \theta \dot{\theta}^{4}+e_{22} \xi(t)
\end{aligned}
$$

$10.5 \mathrm{~m} / \mathrm{s}(11$ terms $): \quad \ddot{\theta}=c_{1} \operatorname{sign}(\dot{\theta})+e_{2} \theta+e_{3} \dot{\theta}+e_{7} \theta^{3}+e_{10} \dot{\theta}^{3}$

$$
\begin{aligned}
& +e_{11} \theta^{4}+e_{16} \theta^{5}+e_{17} \theta^{4} \dot{\theta}+e_{18} \theta^{3} \dot{\theta}^{2} \\
& +e_{20} \theta \dot{\theta}^{4}+e_{21} \dot{\theta}^{5}+e_{22} \xi(t)
\end{aligned}
$$
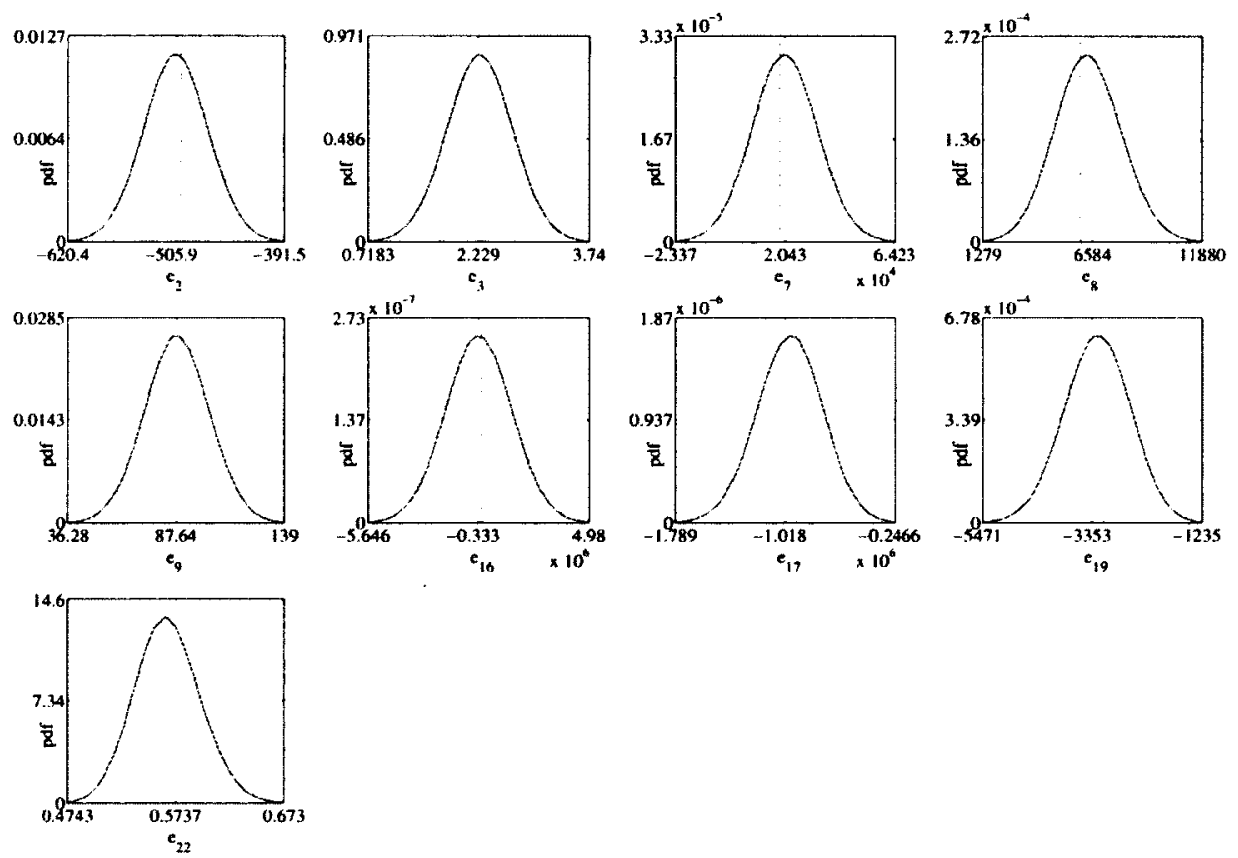

Figure 31: Marginal posterior pdfs and MLE estimates (red-dashed line) for the parameters of the final model for $\operatorname{Re}=75,000$ 


\begin{tabular}{|c|r|r|r|r|r|r|r|r|r|r|r|r|r|r|}
\hline \multirow{2}{*}{ Term } & \multicolumn{10}{|c|}{$\Delta \mathrm{BIC}_{r, G}^{\text {exact }}$ values } \\
\cline { 2 - 3 } & 1 & 2 & 3 & 4 & 5 & 6 & 7 & 8 & 9 & 10 & 11 & 12 & 13 & 14 \\
\hline$e_{1}$ & -0.9 & - & - & - & - & - & - & - & - & - & - & - & - & - \\
\hline$e_{4} \theta^{2}$ & 12.7 & 8.2 & 14.5 & 13.7 & 12.4 & 12.7 & 13.1 & 16.6 & - & - & - & - & - & - \\
\hline$e_{5} \theta \dot{\theta}$ & 7.5 & 4.0 & 9.2 & 8.5 & 9.1 & 8.1 & - & - & - & - & - & - & - & - \\
\hline$e_{6} \dot{\theta}^{2}$ & 1.2 & -2.3 & -2.1 & - & - & - & - & - & - & - & - & - & - & - \\
\hline$e_{8} \theta^{2} \dot{\theta}$ & 20.3 & 17.3 & 22.8 & 20.9 & 21.1 & 20.5 & 19.0 & 19.7 & 19.4 & 17.8 & 32.7 & 32.5 & 31.6 & 32.4 \\
\hline$e_{9} \theta \dot{\theta}^{2}$ & 8.7 & 4.2 & 9.8 & 8.3 & 8.7 & 46.5 & 45.4 & 45.3 & 44.7 & 40.0 & 34.6 & 34.7 & 33.3 & 37.3 \\
\hline$e_{10} \dot{\theta}^{3}$ & 16.4 & 12.2 & 19.4 & 16.7 & 17.5 & 16.0 & 17.1 & 17.3 & 16.0 & - & - & - & - & - \\
\hline$e_{11} \theta^{4}$ & 22.2 & 18.0 & 24.5 & 22.7 & 22.3 & 22.9 & 23.1 & 24.9 & 22.7 & 23.0 & 23.3 & 23.3 & - & - \\
\hline$e_{12} \theta^{3} \dot{\theta}$ & 18.3 & 14.8 & 21.1 & 17.5 & 16.0 & 16.2 & 19.8 & 19.8 & 19.7 & 19.3 & 19.0 & - & - & - \\
\hline$e_{13} \theta^{2} \dot{\theta}^{2}$ & 12.9 & 11.4 & 16.4 & 15.3 & 13.7 & 13.7 & 13.1 & - & - & - & - & - & - & - \\
\hline$e_{14} \theta \dot{\theta}^{3}$ & 7.4 & 4.3 & 9.6 & 6.6 & - & - & - & - & - & - & - & - & - & - \\
\hline$e_{15} \dot{\theta}^{4}$ & -0.5 & -3.8 & - & - & - & - & - & - & - & - & - & - & - & - \\
\hline$e_{16} \theta^{5}$ & 32.4 & 28.2 & 34.2 & 33.6 & 32.3 & 32.1 & 32.6 & 33.0 & 33.8 & 34.0 & 33.7 & 34.5 & 33.3 & 29.3 \\
\hline$e_{17} \theta^{4} \dot{\theta}$ & 30.3 & 26.9 & 32.7 & 31.1 & 32.0 & 32.1 & 31.4 & 30.7 & 30.9 & 30.5 & 44.9 & 44.6 & 43.8 & 44.9 \\
\hline$e_{18} \theta^{3} \dot{\theta}^{2}$ & 19.1 & 14.6 & 20.3 & 18.8 & 15.6 & 24.7 & 25.5 & 25.4 & 26.1 & 24.7 & 23.1 & 23.5 & 22.5 & - \\
\hline$e_{19} \theta^{2} \dot{\theta}^{3}$ & 39.2 & 34.8 & 40.5 & 37.7 & 37.9 & 36.5 & 35.2 & 34.6 & 33.1 & 23.9 & 42.8 & 42.1 & 41.0 & 42.3 \\
\hline$e_{20} \theta \dot{\theta}^{4}$ & 8.4 & 3.7 & 9.9 & 8.9 & 8.4 & - & - & - & - & - & - & - & - & - \\
\hline$e_{21} \dot{\theta}^{5}$ & 20.7 & 16.6 & 20.8 & 22.7 & 21.0 & 20.7 & 20.8 & 33.7 & 19.9 & 9.9 & - & - & - & - \\
\hline
\end{tabular}

Table 12: LCO: $\triangle \mathrm{BIC}_{r, G}^{\text {exact }}$ model selection data for $\operatorname{Re}=75,0000$ 


\begin{tabular}{|c|r|r|r|r|r|r|r|r|r|r|r|r|r|}
\hline \multirow{2}{*}{ Term removed } & \multicolumn{10}{|c|}{$\Delta \mathrm{BIC}_{r, G}^{\text {exact }}$ values } \\
\cline { 2 - 16 } & 1 & 2 & 3 & 4 & 5 & 6 & 7 & 8 & 9 & 10 & 11 & 12 & 13 \\
\hline$e_{1}$ & 0.6 & - & - & - & - & - & - & - & - & - & - & - & - \\
\hline$e_{4} \theta^{2}$ & 13.3 & 10.2 & 17.7 & 13.5 & 14.4 & 13.6 & 26.7 & 30.0 & 27.1 & 27.7 & 28.2 & - & - \\
\hline$e_{5} \theta \dot{\theta}$ & 6.5 & 3.9 & 9.7 & 8.1 & 5.7 & - & - & - & - & - & - & - & - \\
\hline$e_{6} \dot{\theta}^{2}$ & 2.6 & 1.1 & 3.4 & - & - & - & - & - & - & - & - & - & - \\
\hline$e_{8} \theta^{2} \dot{\theta}$ & 13.2 & 9.3 & 15.8 & 13.4 & 14.2 & 13.3 & 14.4 & 15.4 & 16.6 & 15.7 & - & - & - \\
\hline$e_{9} \theta \dot{\theta}^{2}$ & 9.1 & 4.1 & 11.6 & 10.7 & 10.0 & 10.2 & 11.6 & 134.4 & 131.3 & 131.8 & 131.2 & 125.8 & 109.5 \\
\hline$e_{10} \dot{\theta}^{3}$ & 13.4 & 9.2 & 16.1 & 10.3 & 9.9 & 9.9 & 11.9 & 10.2 & - & - & - & - & - \\
\hline$e_{11} \theta^{4}$ & 23.2 & 23.2 & 24.6 & 22.9 & 23.3 & 23.8 & 27.2 & 30.1 & 27.7 & 28.1 & 28.5 & 42.3 & 41.5 \\
\hline$e_{12} \theta^{3} \dot{\theta}$ & 16.9 & 13.5 & 20.0 & 18.5 & 16.4 & 13.6 & 14.8 & 15.2 & 15.6 & - & - & - & - \\
\hline$e_{13} \theta^{2} \dot{\theta}^{2}$ & 12.6 & 6.4 & 15.0 & 10.0 & 10.7 & 9.5 & - & - & - & - & - & - & - \\
\hline$e_{14} \theta \dot{\theta}^{3}$ & 4.5 & 0.4 & 7.2 & 5.1 & - & - & - & - & - & - & - & - & - \\
\hline$e_{15} \dot{\theta}^{4}$ & 1.9 & -1.0 & - & - & - & - & - & - & - & - & - & - & - \\
\hline$e_{16} \theta^{5}$ & 39.3 & 33.7 & 40.7 & 38.4 & 39.9 & 38.0 & 39.4 & 39.5 & 42.1 & 41.8 & 41.6 & 45.2 & 44.1 \\
\hline$e_{17} \theta^{4} \dot{\theta}$ & 25.0 & 22.5 & 25.7 & 24.2 & 24.1 & 23.9 & 24.7 & 25.1 & 25.1 & 25.1 & 65.7 & 60.1 & 35.6 \\
\hline$e_{18} \theta^{3} \dot{\theta}^{2}$ & 24.2 & 19.2 & 25.8 & 24.9 & 25.3 & 26.3 & 27.8 & 63.4 & 63.1 & 63.1 & 62.2 & 61.5 & 55.5 \\
\hline$e_{19} \theta^{2} \dot{\theta}^{3}$ & 27.0 & 21.4 & 29.6 & 25.5 & 24.6 & 23.1 & 24.1 & 22.2 & 17.0 & 20.5 & 115.7 & 106.0 & 83.5 \\
\hline$e_{20} \theta \dot{\theta}^{4}$ & 10.3 & 5.3 & 12.5 & 9.5 & 9.9 & 9.7 & 9.8 & - & - & - & - & - & - \\
\hline$e_{21} \dot{\theta}^{5}$ & 20.4 & 16.2 & 22.9 & 15.1 & 15.5 & 17.8 & 16.9 & 39.5 & 24.2 & 24.1 & 29.1 & 22.9 & - \\
\hline
\end{tabular}

Table 13: $\mathrm{LCO}: \Delta \mathrm{BIC}_{r, G}^{\text {exact }}$ model selection data for $\mathrm{Re}=85,0000$ 


\begin{tabular}{|c|r|r|r|r|r|r|r|r|r|r|r|r|}
\hline Term & \multicolumn{10}{|c|}{$\Delta$ BIC $C_{r, G}^{\text {exact }}$ values } \\
\hline & 1 & 2 & 3 & 4 & 5 & 6 & 7 & 8 & 9 & 10 & 11 & 12 \\
\hline$e_{1}$ & -1.6 & - & - & - & - & - & - & - & - & - & - & - \\
\hline$e_{4} \theta^{2}$ & 12.3 & 9.4 & 14.8 & 13.6 & 12.5 & 12.0 & 13.7 & 39.5 & 39.4 & 39.7 & 34.6 & 29.1 \\
\hline$e_{5} \theta \dot{\theta}$ & 6.8 & 7.9 & 9.6 & 6.9 & 10.1 & 10.3 & - & - & - & - & - & - \\
\hline$e_{6} \dot{\theta}^{2}$ & 0.8 & -2.1 & 2.9 & - & - & - & - & - & - & - & - & - \\
\hline$e_{8} \theta^{2} \dot{\theta}$ & 16.5 & 11.7 & 16.6 & 13.7 & 14.6 & 13.7 & 14.9 & 15.6 & - & - & - & - \\
\hline$e_{9} \dot{\theta}^{2}$ & 8.3 & 5.0 & 11.1 & 9.1 & 7.6 & - & - & - & - & - & - & - \\
\hline$e_{10} \dot{\theta}^{3}$ & 22.7 & 18.9 & 24.7 & 17.3 & 18.2 & 17.7 & 21.5 & 20.2 & 21.9 & 21.0 & - & - \\
\hline$e_{11} \theta^{4}$ & 22.6 & 19.1 & 24.7 & 24.1 & 22.3 & 22.3 & 23.3 & 35.6 & 36.0 & 35.8 & 32.7 & 29.6 \\
\hline$e_{12} \theta^{3} \dot{\theta}$ & 17.4 & 14.4 & 20.1 & 18.0 & 18.0 & 17.5 & 18.1 & 17.0 & 17.9 & - & - & - \\
\hline$e_{13} \theta^{2} \dot{\theta}^{2}$ & 13.1 & 10.2 & 14.9 & 15.5 & 14.2 & 13.8 & 13.4 & - & - & - & - & - \\
\hline$e_{14} \theta \dot{\theta}^{3}$ & 5.2 & 2.7 & 7.9 & 5.2 & - & - & - & - & - & - & - & - \\
\hline$e_{15} \dot{\theta}^{4}$ & -0.3 & -4.6 & - & - & - & - & - & - & - & - & - & - \\
\hline$e_{16} \theta^{5}$ & 42.7 & 40.3 & 45.8 & 43.0 & 42.7 & 43.2 & 43.4 & 41.8 & 42.8 & 42.5 & 43.1 & 42.8 \\
\hline$e_{17} \theta^{4} \dot{\theta}$ & 23.8 & 20.9 & 26.5 & 24.2 & 24.9 & 24.9 & 24.5 & 25.8 & 23.0 & 23.8 & 49.8 & 28.4 \\
\hline$e_{18} \theta^{3} \dot{\theta}^{2}$ & 21.8 & 18.3 & 24.4 & 23.4 & 22.9 & 42.4 & 42.3 & 41.0 & 42.8 & 42.8 & 39.1 & 37.3 \\
\hline$e_{19} \theta^{2} \dot{\theta}^{3}$ & 37.0 & 35.3 & 39.4 & 35.7 & 31.5 & 36.4 & 34.1 & 33.7 & 144.3 & 143.1 & 152.1 & 127.6 \\
\hline$e_{20} \theta \dot{\theta}^{4}$ & 24.7 & 22.1 & 24.1 & 23.3 & 23.5 & 188.2 & 184.1 & 183.9 & 185.2 & 183.7 & 168.6 & 148.6 \\
\hline$e_{21} \dot{\theta}^{5}$ & 29.0 & 25.8 & 30.2 & 23.0 & 22.9 & 23.2 & 29.0 & 27.2 & 28.1 & 27.2 & 23.3 & - \\
\hline
\end{tabular}

Table 14: LCO: $\Delta \mathrm{BIC}_{r, G}^{\text {exact }}$ model selection data for $\mathrm{Re}=90,0000$ 


\begin{tabular}{|c|c|c|c|c|c|c|c|c|c|c|c|c|}
\hline \multirow{2}{*}{ Term } & \multicolumn{12}{|c|}{$\Delta \mathrm{BIC}_{r, G}^{\text {exact }}$ values } \\
\hline & 1 & 2 & 3 & 4 & 5 & 6 & 7 & 8 & 9 & 10 & 11 & 12 \\
\hline$e_{1}$ & -1.2 & -4.8 & - & - & - & - & - & - & - & - & - & - \\
\hline$e_{4} \theta^{2}$ & 14.2 & 9.7 & 15.0 & 12.8 & 12.2 & 12.9 & 13.5 & 14.6 & 14.6 & - & - & . \\
\hline$e_{5} \theta \dot{\theta}$ & 9.2 & 5.5 & 10.9 & 8.3 & 4.8 & - & - & - & - & - & - & . \\
\hline$e_{6} \dot{\theta}^{2}$ & 0.9 & 2.0 & 6.3 & - & - & - & - & - & - & - & - & . \\
\hline$e_{8} \theta^{2} \dot{\theta}$ & 15.8 & 10.9 & 16.8 & 14.7 & 14.0 & 14.5 & 15.4 & 16.5 & 14.6 & 14.8 & - & - \\
\hline$e_{9} \theta \dot{\theta}^{2}$ & 12.8 & 9.3 & 15.5 & 11.8 & 12.5 & 12.5 & 12.6 & - & - & - & - & . \\
\hline$e_{10} \dot{\theta}^{3}$ & 19.2 & 15.7 & 21.5 & 16.5 & 15.9 & 17.5 & 19.0 & 22.4 & 24.1 & 23.8 & 29.9 & 29.5 \\
\hline$e_{11} \theta^{4}$ & 25.5 & 21.6 & 27.3 & 24.4 & 24.0 & 24.6 & 29.6 & 30.2 & 30.4 & 47.6 & 47.3 & 47.1 \\
\hline$e_{12} \theta^{3} \dot{\theta}$ & 20.5 & 22.0 & 23.3 & 19.7 & 18.8 & 17.7 & 18.2 & 18.9 & 18.8 & 19.0 & 18.9 & - \\
\hline$e_{13} \theta^{2} \dot{\theta}^{2}$ & 15.3 & 10.9 & 16.7 & 11.3 & 10.8 & 12.3 & - & - & - & - & - & - \\
\hline$e_{14} \theta \dot{\theta}^{3}$ & 6.3 & 2.9 & 9.6 & 7.7 & - & - & - & - & - & - & - & 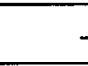 \\
\hline$e_{15} \dot{\theta}^{4}$ & -1.4 & - & - & - & - & - & - & - & - & - & - & - \\
\hline$e_{16} \theta^{5}$ & 41.9 & 37.9 & 45.1 & 41.1 & 40.8 & 41.8 & 42.8 & 42.1 & 42.2 & 41.1 & 41.4 & 41.1 \\
\hline$e_{17} \theta^{4} \dot{\theta}$ & 30.9 & 26.3 & 33.6 & 32.1 & 30.4 & 31.0 & 32.3 & 35.0 & 42.9 & 42.8 & 120.2 & 118.6 \\
\hline$e_{18} \theta^{3} \dot{\theta}^{2}$ & 49.6 & 46.6 & 52.9 & 49.2 & 48.5 & 48.5 & 50.6 & 67.8 & 75.7 & 75.1 & 76.1 & 75.4 \\
\hline$e_{19} \theta^{2} \dot{\theta}^{3}$ & 12.5 & 9.9 & 15.6 & 12.5 & 12.3 & 13.1 & 13.9 & 14.2 & - & - & - & \\
\hline$e_{20} \theta \dot{\theta}^{4}$ & 10.3 & 7.3 & 13.2 & 11.2 & 11.8 & 12.6 & 13.2 & 145.0 & 147.1 & 146.0 & 154.1 & 153.7 \\
\hline$e_{21} \dot{\theta}^{5}$ & 28.0 & 25.6 & 30.9 & 23.1 & 22.8 & 26.6 & 28.0 & 32.1 & 32.4 & 32.7 & 43.7 & 42.8 \\
\hline
\end{tabular}

Table 15: LCO: $\triangle \mathrm{BIC}_{r, G}^{\text {exact }}$ model selection data for $\mathrm{Re}=105,0000$ 


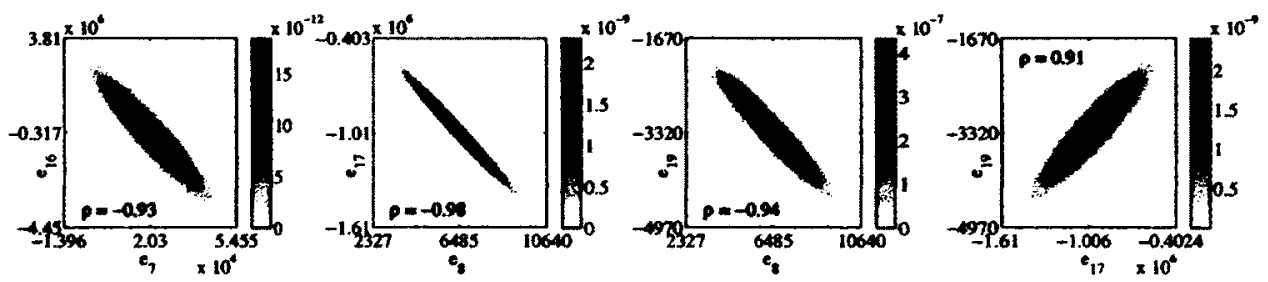

Figure 32: Selected joint posterior pdfs for the parameters of the final model for Re $=75,000$
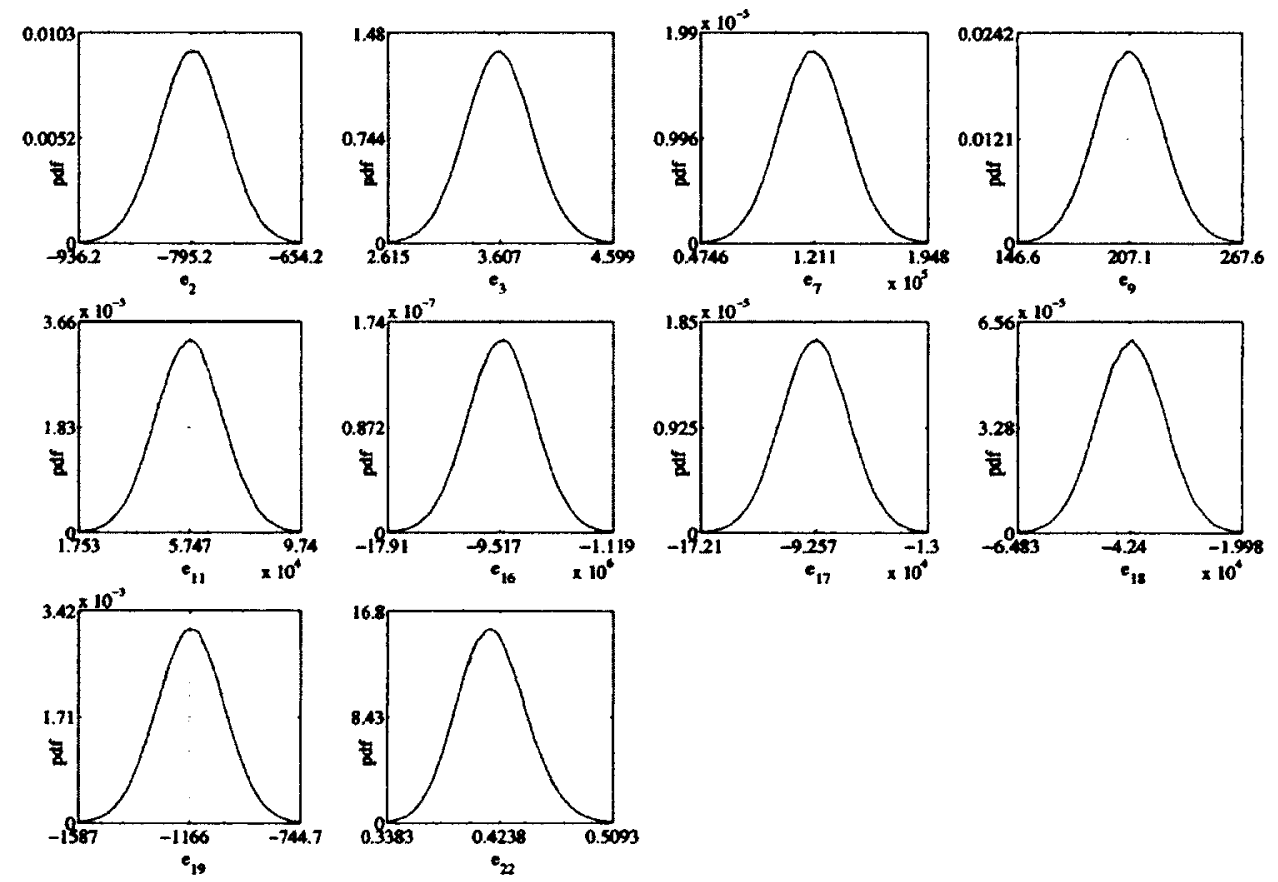

Figure 33: Marginal posterior pdfs and MLE estimates (red-dashed line) for the parameters of the final model for $\operatorname{Re}=85,000$ 

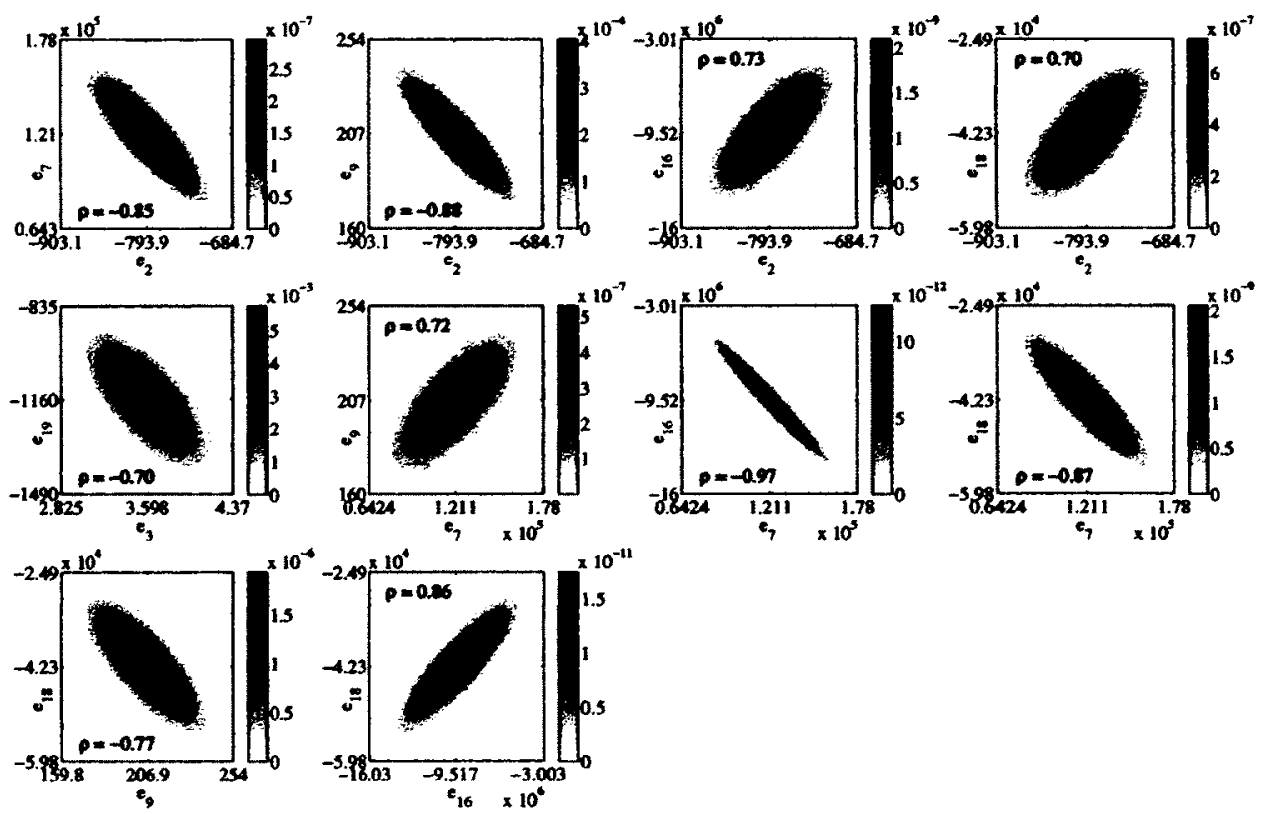

Figure 34: Selected joint posterior pdfs for the parameters of the final model for $R e$ $=85,000$
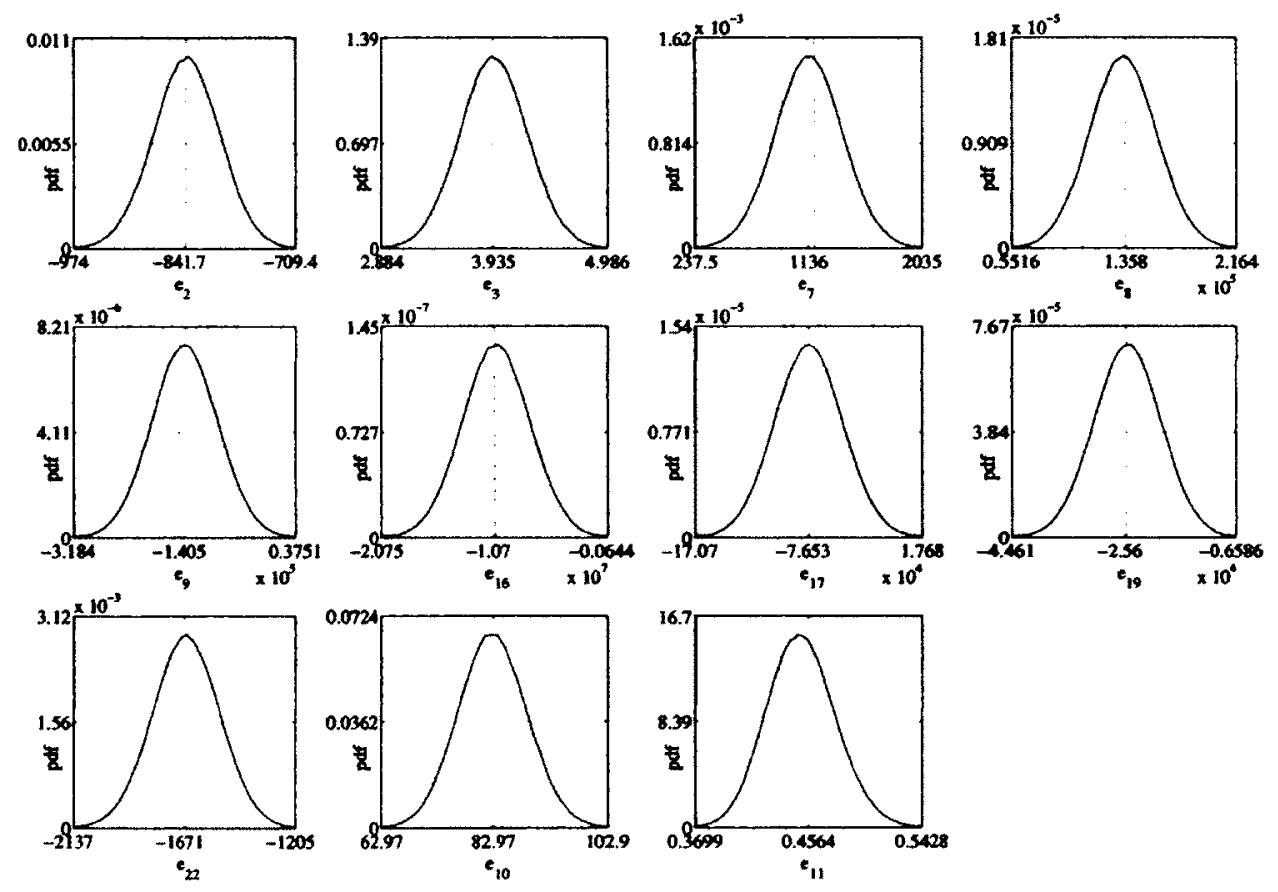

Figure 35: Marginal posterior pdfs and MLE estimates (red-dashed line) for the parameters of the final model for $\operatorname{Re}=90,000$ 

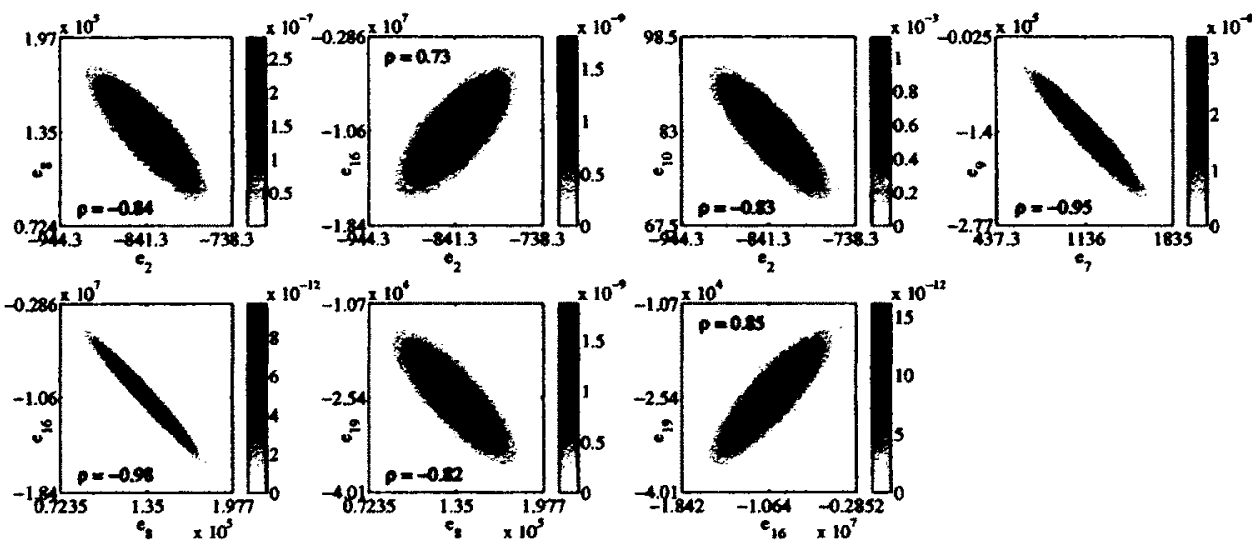

Figure 36: Selected joint posterior pdfs for the parameters of the final model for $R e$ $=90,000$
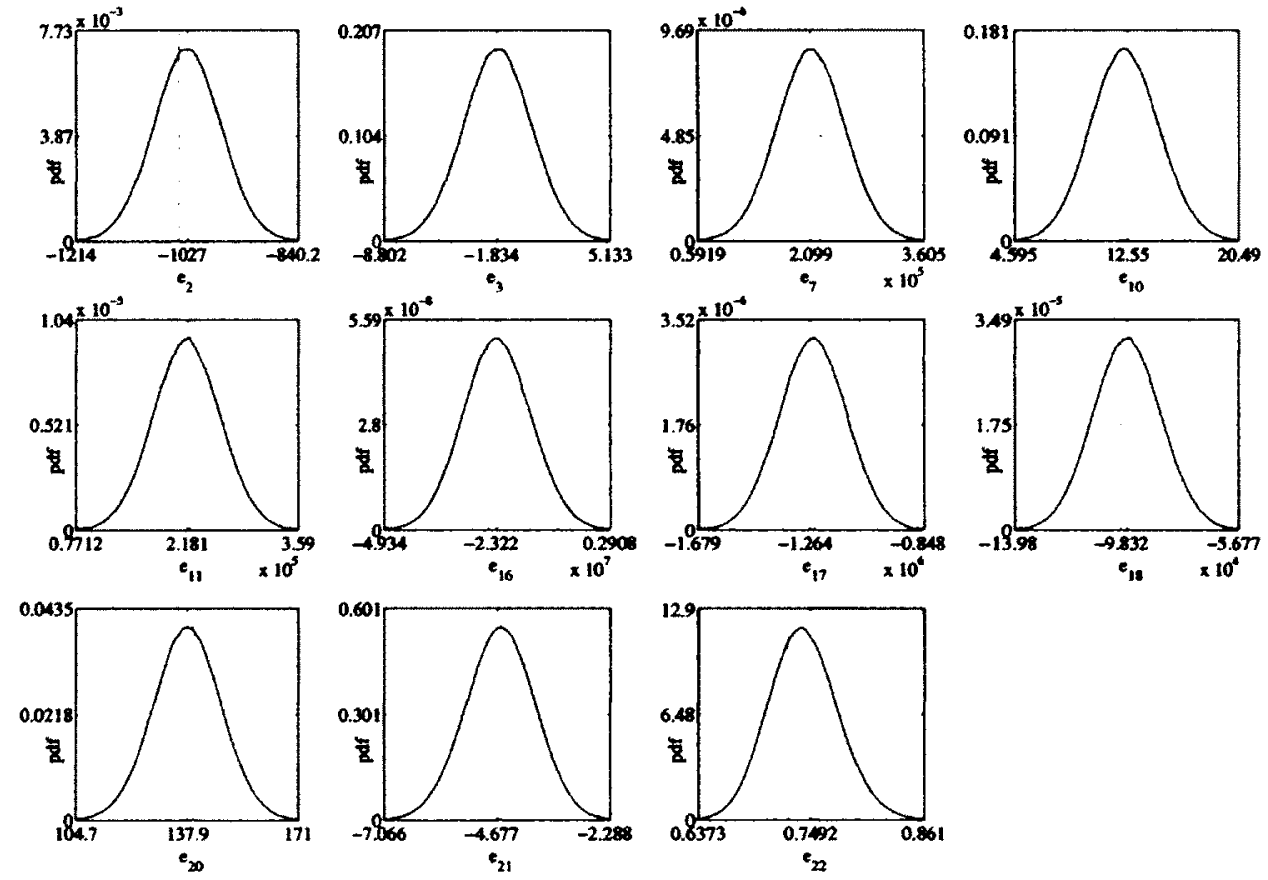

Figure 37: Marginal posterior pdfs and MLE estimates (red-dashed line) for the parameters of the final model for $\operatorname{Re}=105,000$ 

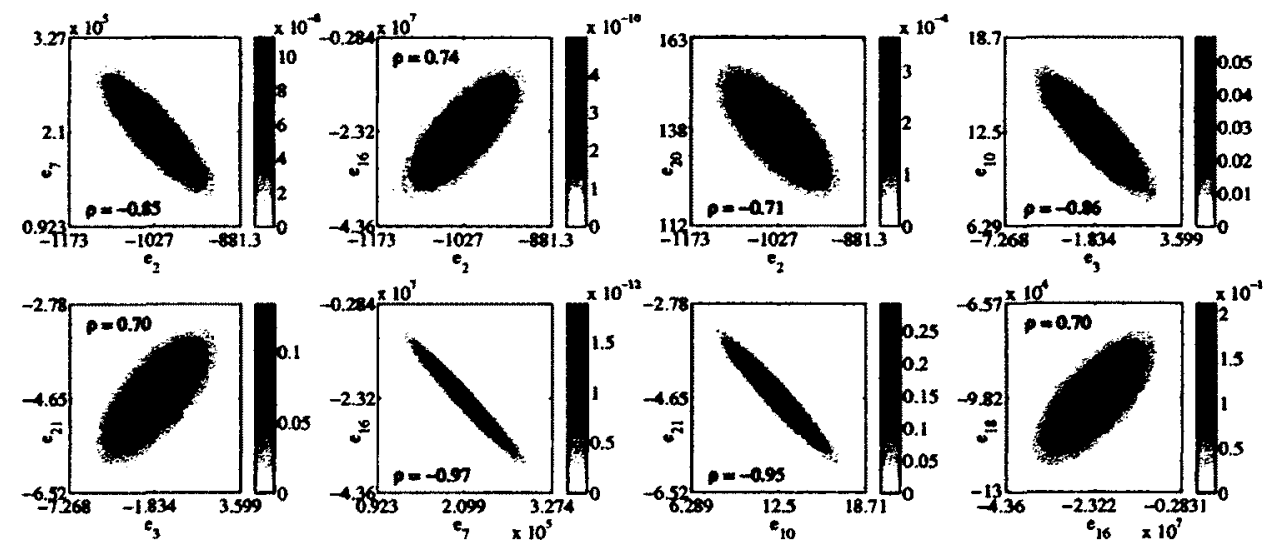

Figure 38: Selected joint posterior pdfs for the parameters of the final model for Re $=105,000$

\subsubsection{Important observations}

The following observations are made from the modelling of the LCO response for four Re values:

\section{The final chosen model}

The increase in number of terms in the final chosen model with increasing Re may imply more complex physics with increasing Re. Also notice that most of the even terms $\left(e_{1}, e_{4} \theta^{2}, e_{5} \theta \dot{\theta}, e_{6} \dot{\theta}^{2}, e_{11} \theta^{4}, e_{12} \theta^{3} \dot{\theta}, e_{13} \theta^{2} \dot{\theta}^{2}, e_{14} \theta \dot{\theta}^{3}, e_{15} \dot{\theta}^{4}\right)$ are missing from the final chosen model, except in few cases, which implies that the non-symmetric aerodynamic moment introduced by the misalignment of the wing is negligible comparable to the symmetric moment created by the formation of LSB [5].

\section{Variation of static pitching moment coefficient with AOA}

The estimated static aerodynamic moment coefficient $C_{M}$ is obtained for each model as

$$
C_{M}=\frac{M_{a e}}{1 / 2 \rho U^{2} s c^{2}}
$$


where $U\left(7.5,8.5,9.0\right.$ and 10.5) is the airspeed, $\rho\left(=1.19 \mathrm{~kg} / \mathrm{m}^{3}\right)$ is the density of air and $M_{e a}$ is the static aerodynamic moment obtained by using the MCMC posterior samples of parameters $e_{1}$ through $e_{2} 1$ (for the final model in Equations (95) to (98)), MLE estimates of $c_{1}$ through $c_{1} 1$ (for the final model in Eq. (92)) and putting the pitch velocity $\dot{\theta}$ term to zero. The structural contributions $\left(c_{1}\right.$ through $\left.c_{1} 1\right)$ are subtracted from the aeroelastic physics $\left(e_{1}\right.$ through $\left.e_{2} 1\right)$ to obtain the aerodynamic contributions of the oscillations. Since even terms are insignificant to the flow aerodynamics, we only consider terms with odd powers of $\theta$ for calculating $M_{a e}$. For example, the expression for $C_{M}$ for $\operatorname{Re}_{c}=85,000$ (or $U=8.5$ ) will be

$$
C_{M}=\frac{\left(e_{2}-c_{3}\right) \theta+\left(e_{7}-c_{8}\right) \theta^{3}+e_{16} \theta^{5}}{2 \cdot(1.19) \cdot(7.5)^{2} \cdot(0.61) \cdot(0.156)^{2}}
$$

Figures 39 to 42 shows the $C_{M}$ variations with the AOA for various $\operatorname{Re}$ values. The $C_{M}$ values are also contrasted with the static aerodynamic moment coefficient obtained by Huang et al. [94] for a NACA0012 cantilever wing at Re $=77,000$ and $\operatorname{Re}=100,000$ with the moment taken at the quarter chord point. The nonlinear variation of the $C_{M}$ with the $\mathrm{AOA}$ is conclusive and is in fair agreement with Huang et al. findings. The variation of the nonlinearity in $C_{M}$ obtained using the MLE estimates is shown in Fig. 43 for the four Re values. Note that differences are expected between our $C_{M}$ results and that of Huang et al., for their position of $E A$ is different (at c/4) and the NACA0012 wing is cantilever.

\section{The predicted response realizations}

The response realizations were generated by using the final chosen models and their MLE parameter estimates. Figures 44 to 47 shows the predicted response realizations in relation to their original response (filtered). It shows that the predicted response 


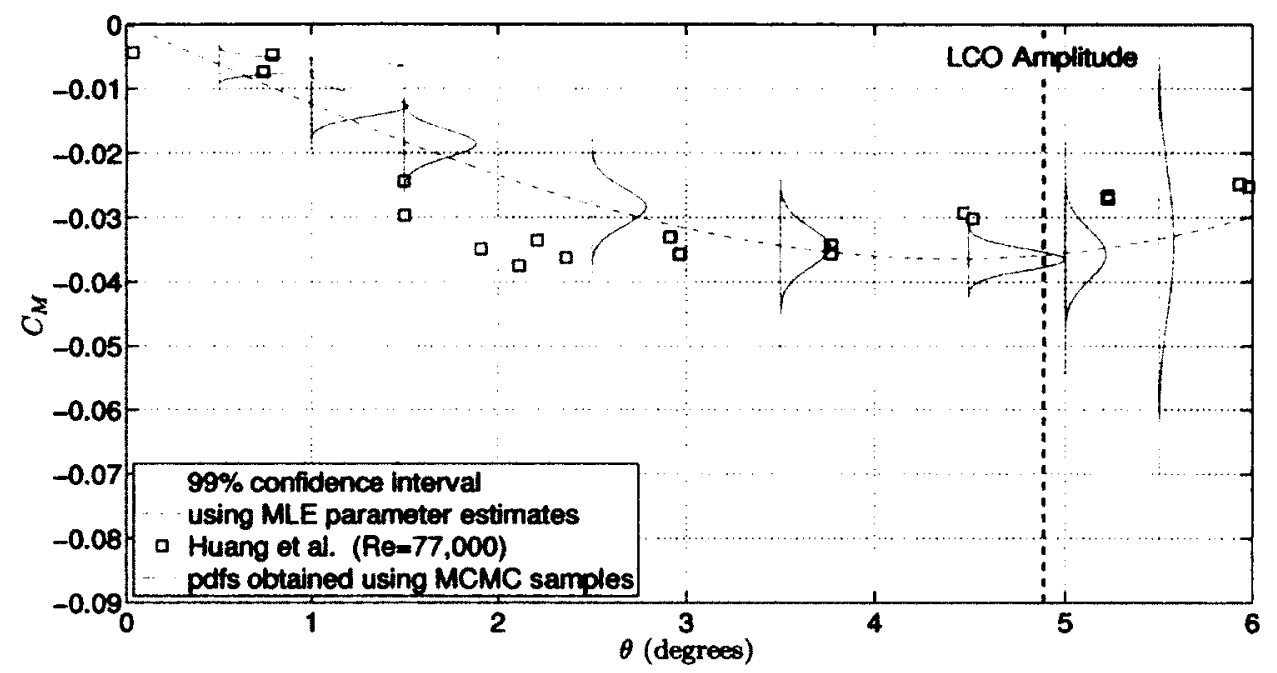

Figure 39: Static moment coefficient for $\operatorname{Re}=75,000$

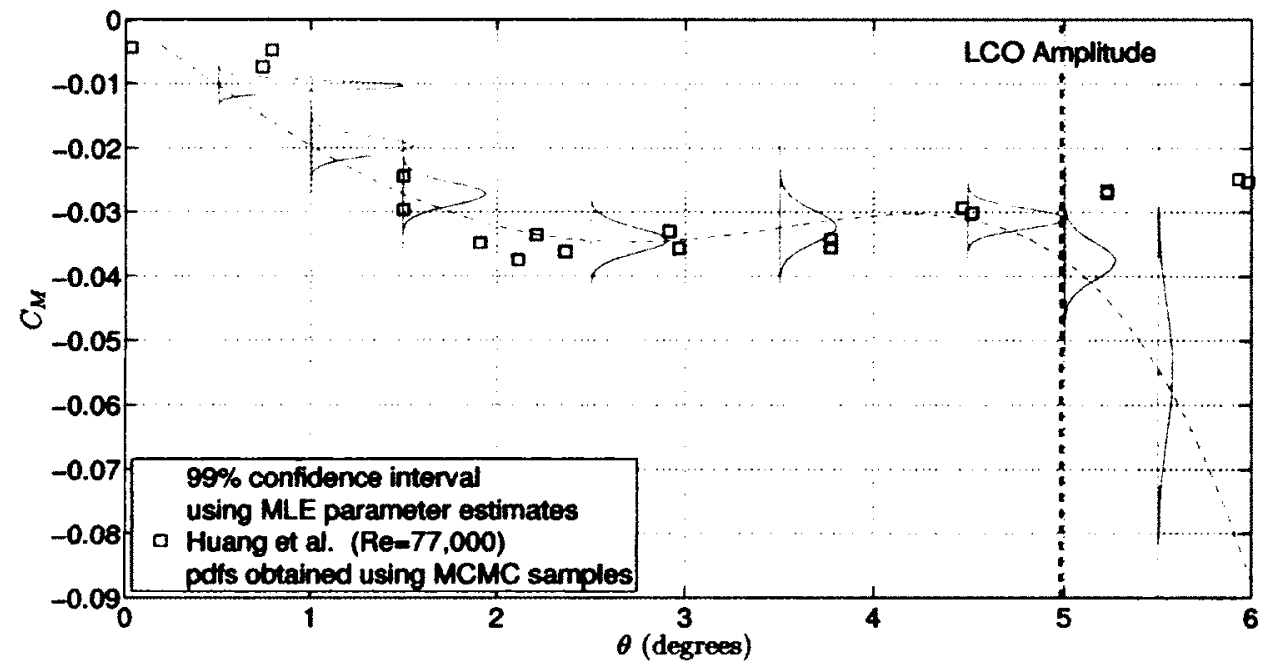

Figure 40: Static moment coefficient for $\operatorname{Re}=85,000$ 


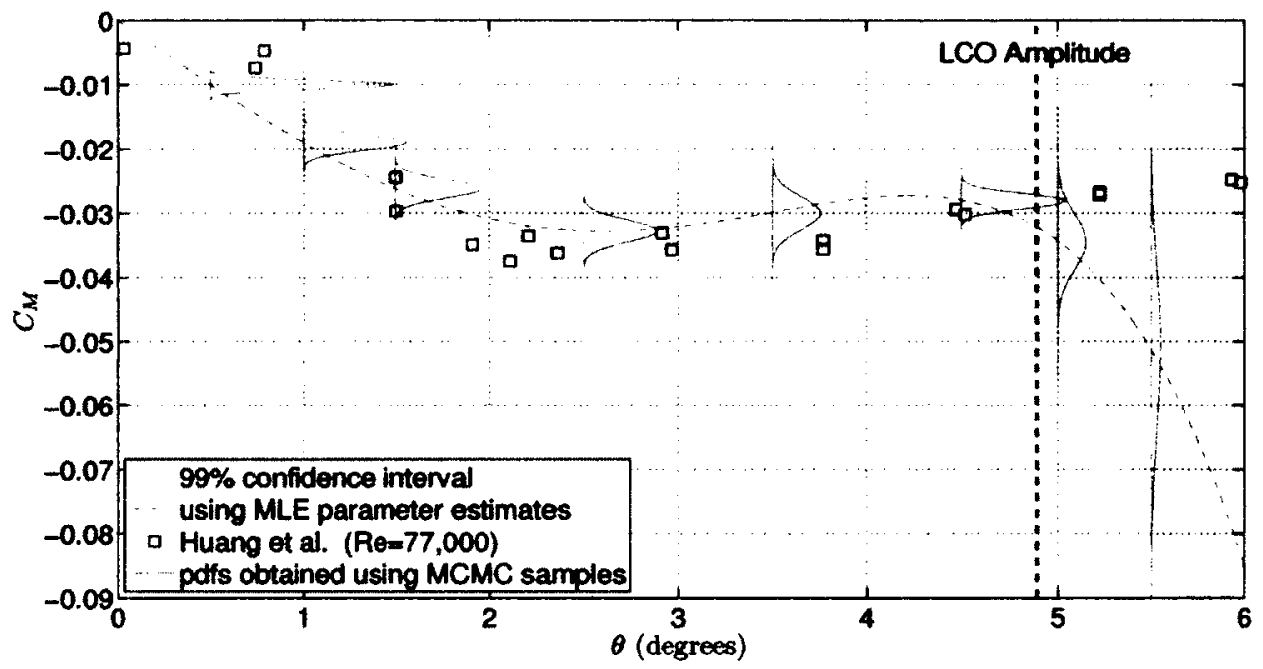

Figure 41: Static moment coefficient for $\operatorname{Re}=90,000$

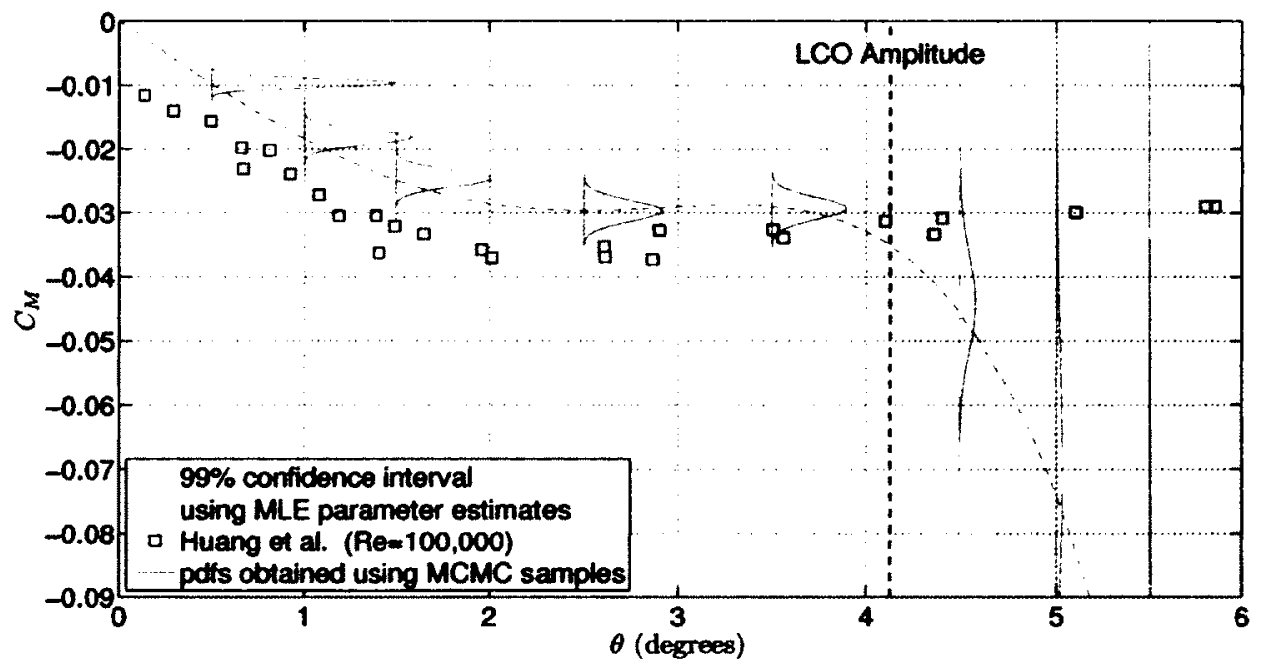

Figure 42: Static moment coefficient for $R e=105,000$ 


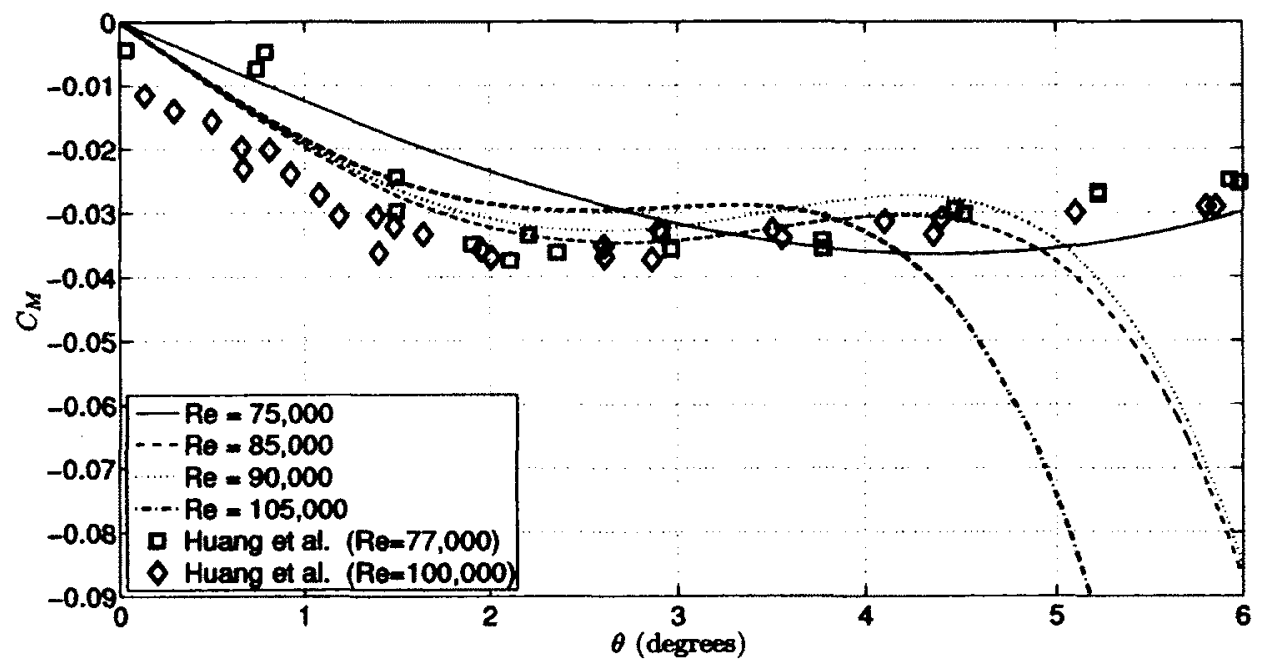

Figure 43: Static moment coefficient using MLE estimates for different Re values

matches reasonably well with the filtered response. The variation between the realizations is due to the modelling noise.

\section{Comparison with the nonlinear unsteady aerodynamics}

The unsteady effect of the trailing vortices on the wing motion can be explained through following argument. An oscillating wing continuously sheds a starting vortex from its trailing edge. A vortex is defined as a fluid swirling rapidly around a core. This starting vortex is originating due to the change of the AOA. As the AOA changes, the circulation around the wing also changes and a starting vortex is formed at the trailing edge with a circulation equal to the negative of the change in the circulation around the airfoil. This ensures that the net circulation around the airfoil remains constant (Kelvin's circulation theorem [96]). This negative circulation vortex affects the vertical velocity around the airfoil and therefore effects the lift around the airfoil. However, the vortex moves downstream with the flow and its effects reduces with time. 

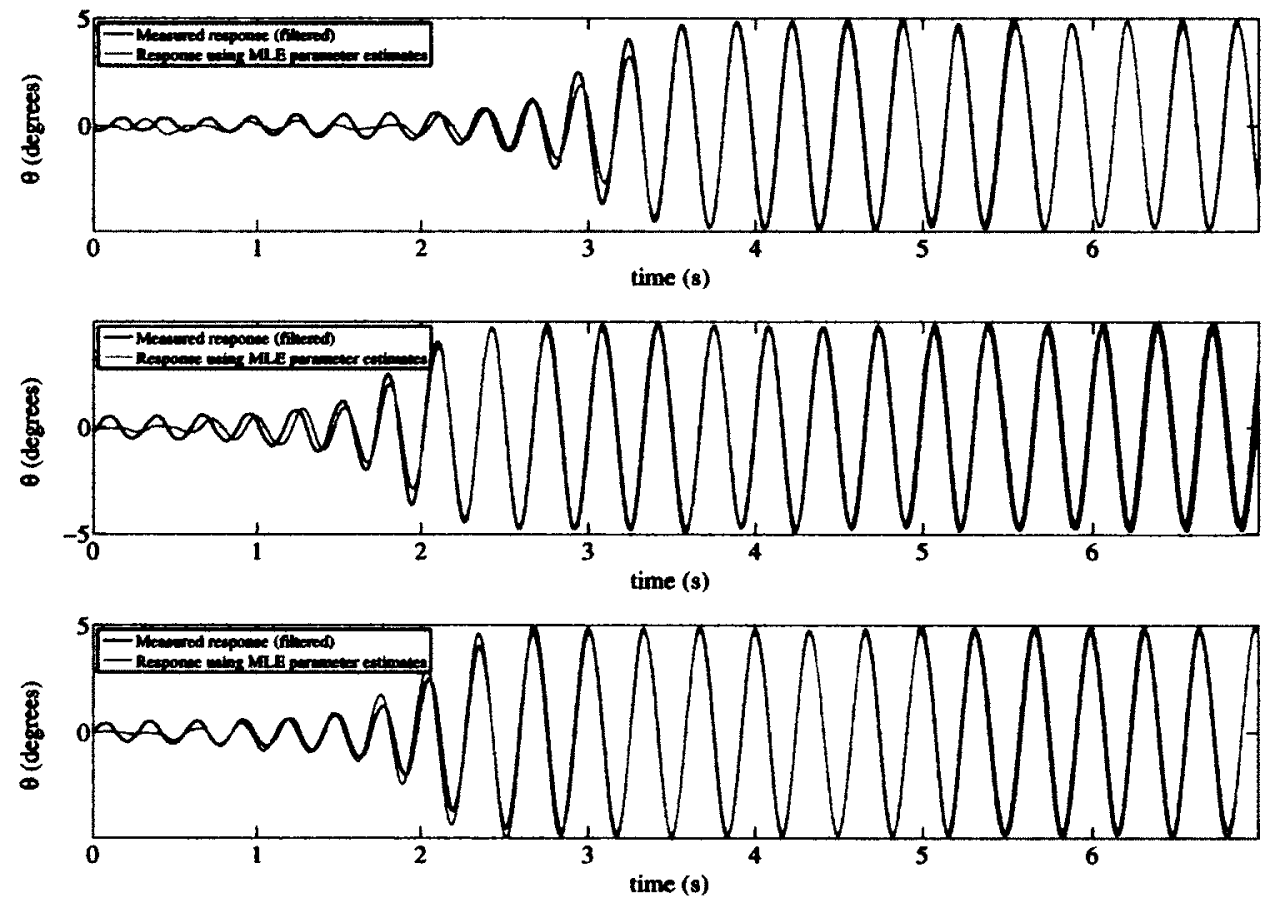

Figure 44: Comparison of response realizations obtained using the final chosen model with the filtered response for $\operatorname{Re}=75,000$ 

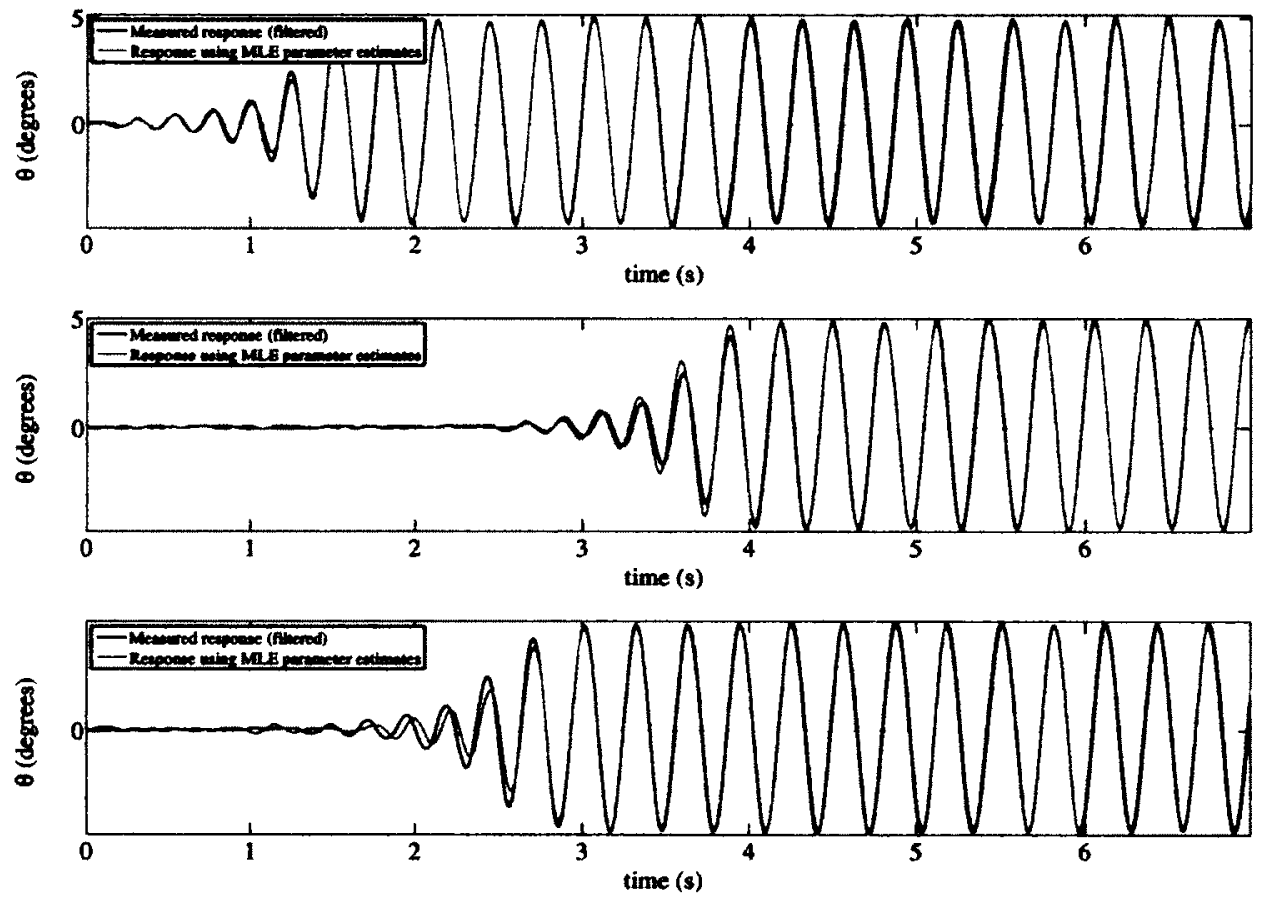

Figure 45: Comparison of response realization obtained using the final chosen model with the filtered response for $\operatorname{Re}=85,000$ 

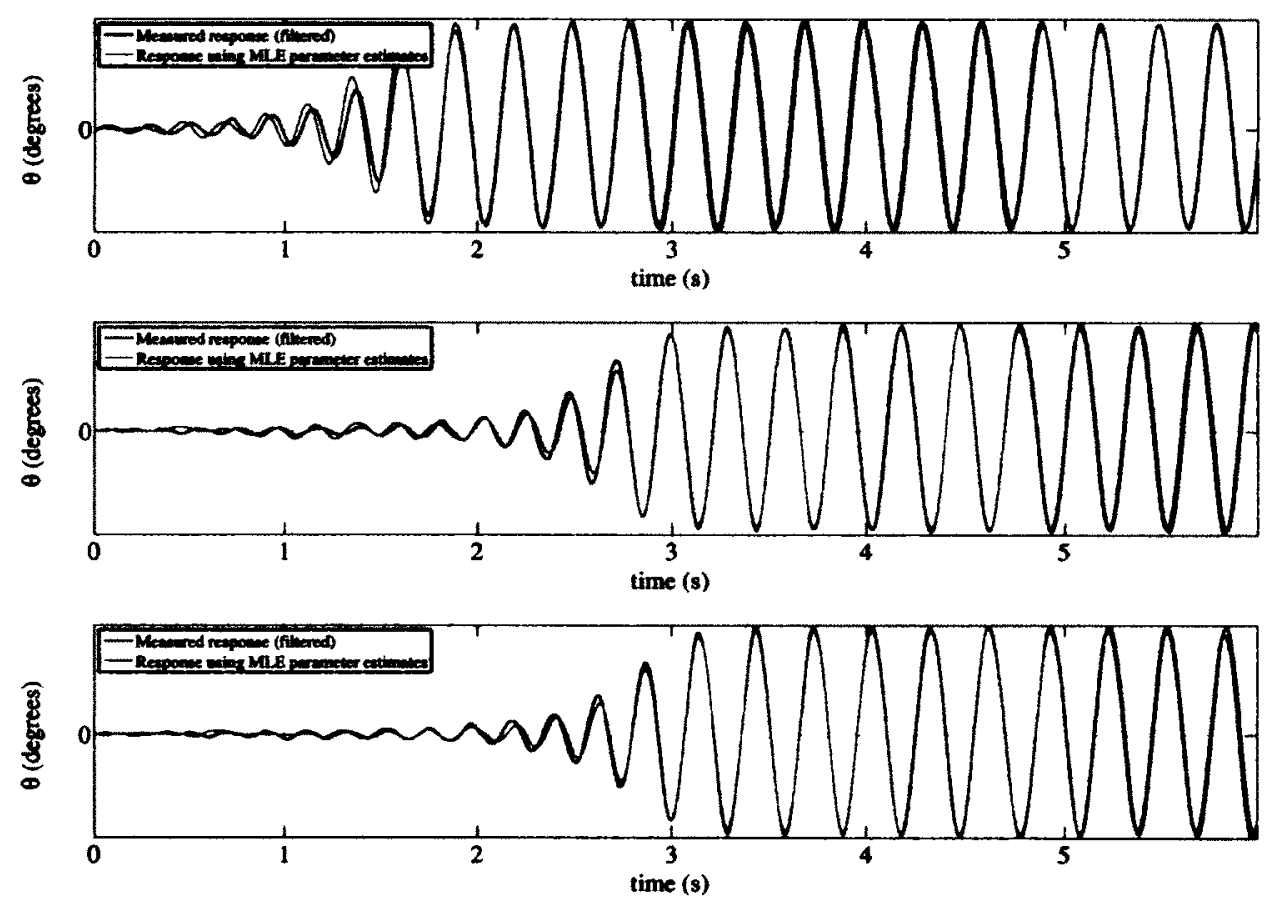

Figure 46: Comparison of response realization obtained using the final chosen model with the filtered response for $\operatorname{Re}=90,000$ 

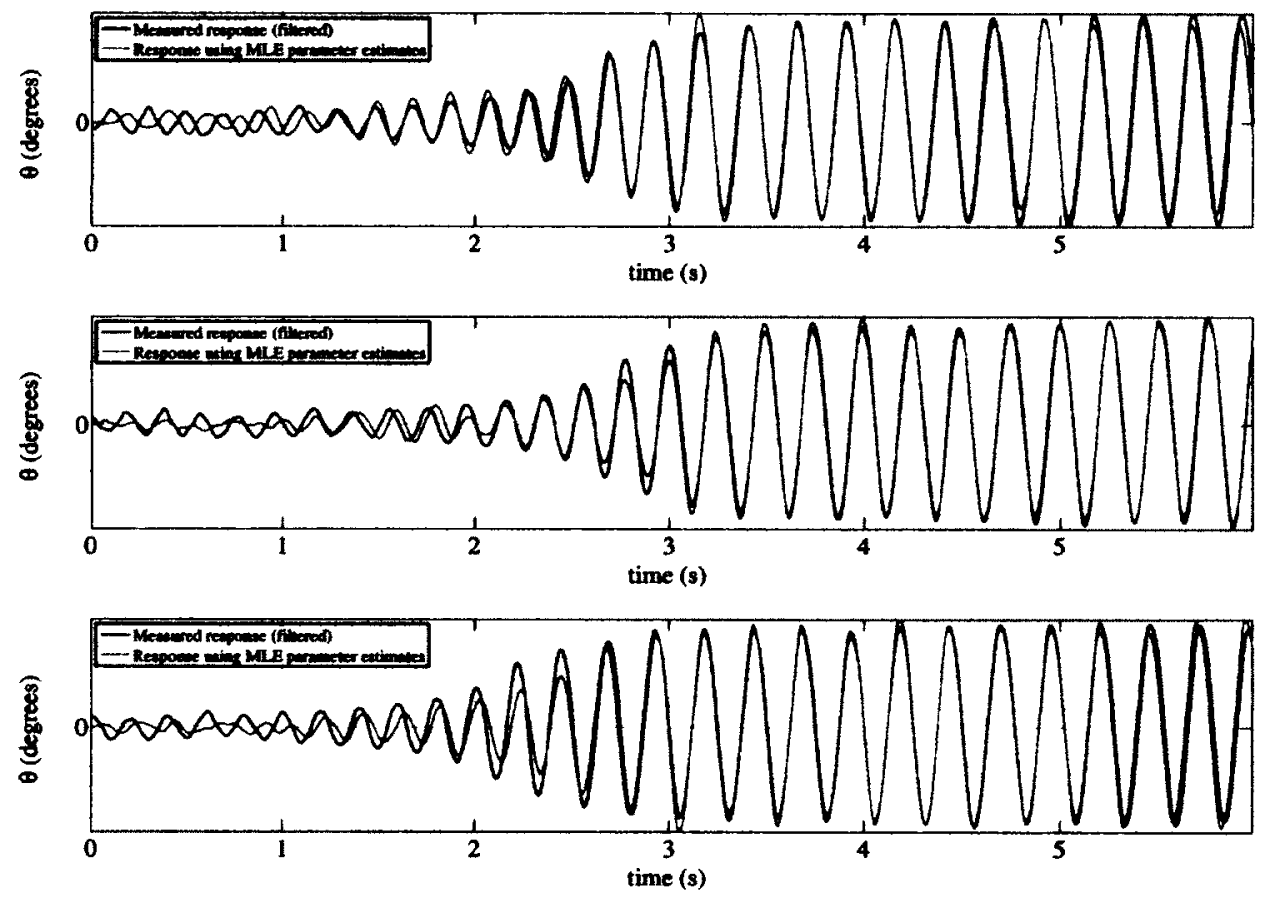

Figure 47: Comparison of response realization obtained using the final chosen model with the filtered response for $\operatorname{Re}=105,000$ 
The unsteady aerodynamic forces around the airfoil can be modeled by the Wagner's function $\varphi(\tau)$ in case of the time domain analysis [96]. The Wagner function states that the lift is half of its actual value when the starting vortex is at the trailing edge and starts reaching its steady state value while the vortex moves farther in the downstream. The Wagner's function $\varphi(\tau)$ captures the diminishing affect of a shedding vortex on the lift around the wing as a function of dimensionless time $\tau=2 U t / c$. The unsteady aerodynamics of a continuously moving wing can be modeled by the superposition of several impulsive changes in the AOA.

Several approximation of the Wagner's function are available in the literature, most notably the two-state representation given as

$$
\varphi(\tau)=1-0.165 e^{-.0455 \tau}-0.335 e^{0.3 \tau}
$$

Fig. 48 shows the Wagner's function. Since the Wagner's model of unsteady aerodynamics is based on the linear thin-airfoil theory, it cannot be applied to the current problem in its given form.

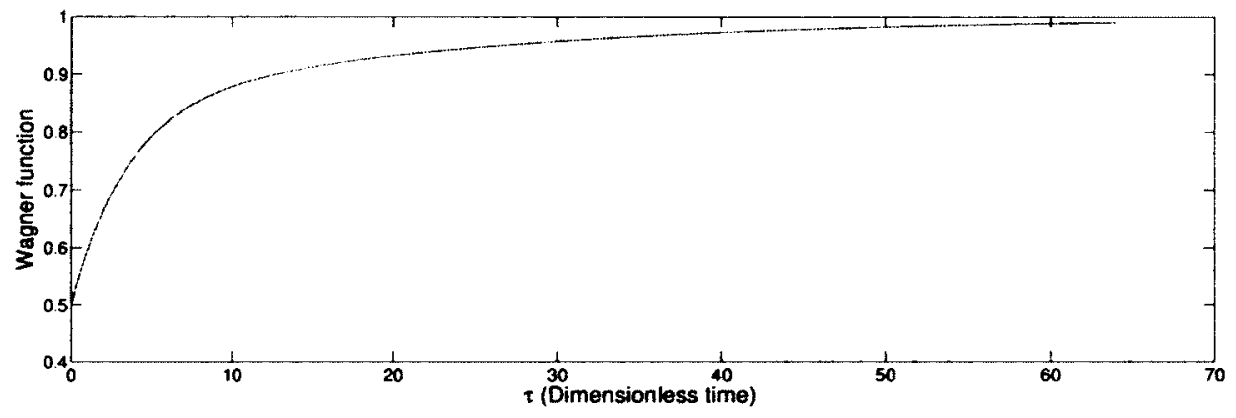

Figure 48: Wagner's function

The effect of unsteady aerodynamics was studied by proposing a nonlinear unsteady model obtained using Wagner's first order linear unsteady aerodynamics [96]. 
The model proposed was

$$
I \ddot{\theta}+D \dot{\theta}+K \theta+K^{\prime} \theta^{3}+c^{\prime} \operatorname{sign}(\dot{\theta})=M(\theta, \dot{\theta}, \ddot{\theta})+\sigma \xi(t)
$$

where the aerodynamics moment $M$ is modeled as a $1^{\text {st }}$ order ODE forced by a generalized $5^{\text {th }}$ order polynomial, as follows

$$
\begin{aligned}
\dot{M}+b M= & b_{1}+b_{2} \theta+b_{3} \dot{\theta}+b_{4} \theta^{2}+b_{5} \theta \dot{\theta}+b_{6} \dot{\theta}^{2} \\
& +b_{7} \theta^{3}+b_{8} \theta \dot{\theta}^{2}+b_{9} \theta^{2} \dot{\theta}+b_{10} \dot{\theta}^{3}+b_{11} \theta^{4}+b_{12} \theta^{3} \dot{\theta} \\
& +b_{13} \theta^{2} \dot{\theta}^{2}+b_{14} \theta \dot{\theta}^{3}+b_{15} \dot{\theta}^{4}+b_{16} \theta^{5}+b_{17} \theta^{4} \dot{\theta}+ \\
& +b_{18} \theta^{3} \dot{\theta}^{2}+b_{19} \theta^{2} \dot{\theta}^{3}+b_{20} \theta \dot{\theta}^{4}+b_{21} \dot{\theta}^{5}+b_{22} \ddot{\theta}+b_{23} \xi(t)
\end{aligned}
$$

It can be noticed that the generalized quasi-steady model considered previously is a special case of the above model. In particular, for very large $b$ value the effect of the term $\dot{M}$ is negligible, thereby reducing it to the generalized quasi-steady model. In fact, a lower value of coefficient $b$ is an indicator of the unsteady effects. We estimate the parameters of this model for all four Re responses. The static aerodynamic moment coefficient for the generalized quasi-steady model and the generalized unsteady model is shown in Figures 49 to 52

The $C_{M}$ values for the unsteady and the quasi-steady models are nearly same for all $\operatorname{Re}$ cases except at $\operatorname{Re}=105,000$. This observation is in agreement with the arguments made by Poirel et al. [85] and Poirel and Yuan [3]. Further insight can be obtained by the comparison of the MLE estimates of the coefficient of $M$ in Eq. (103) for the four Re values, which are shown in Table 16. 


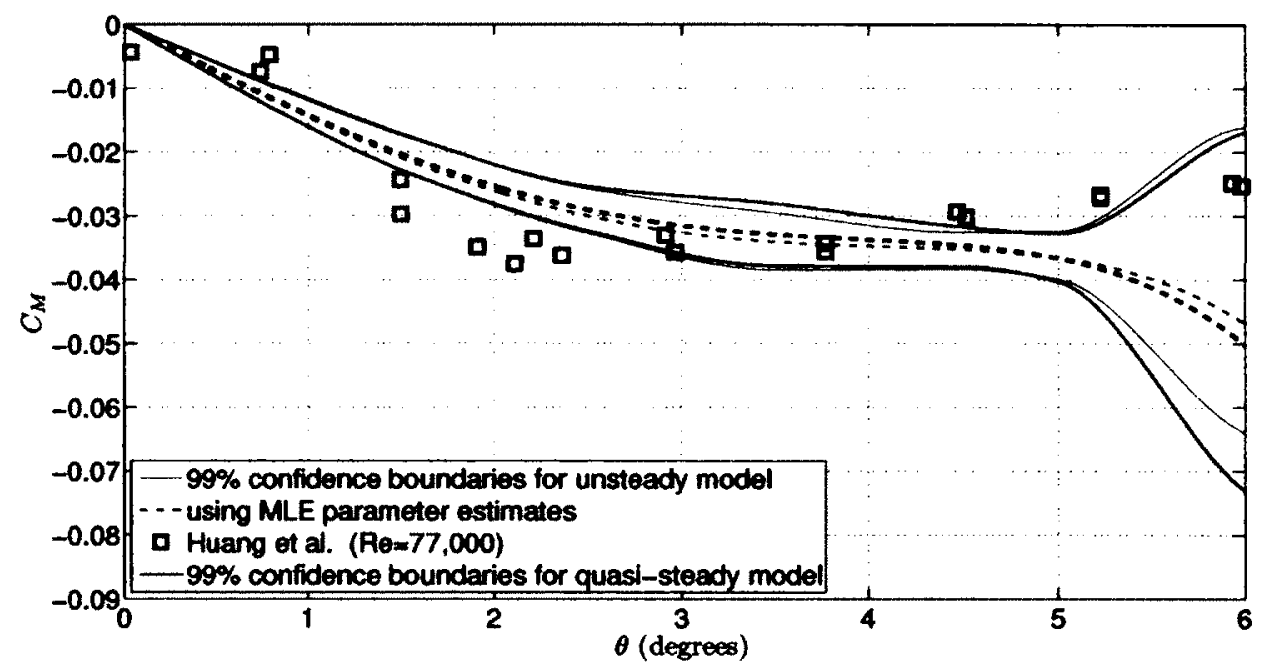

Figure 49: Comparison of the static aerodynamic moment coefficient for the quasisteady and the unsteady $5^{\text {th }}$ order generalized models for $\operatorname{Re}=75,000$

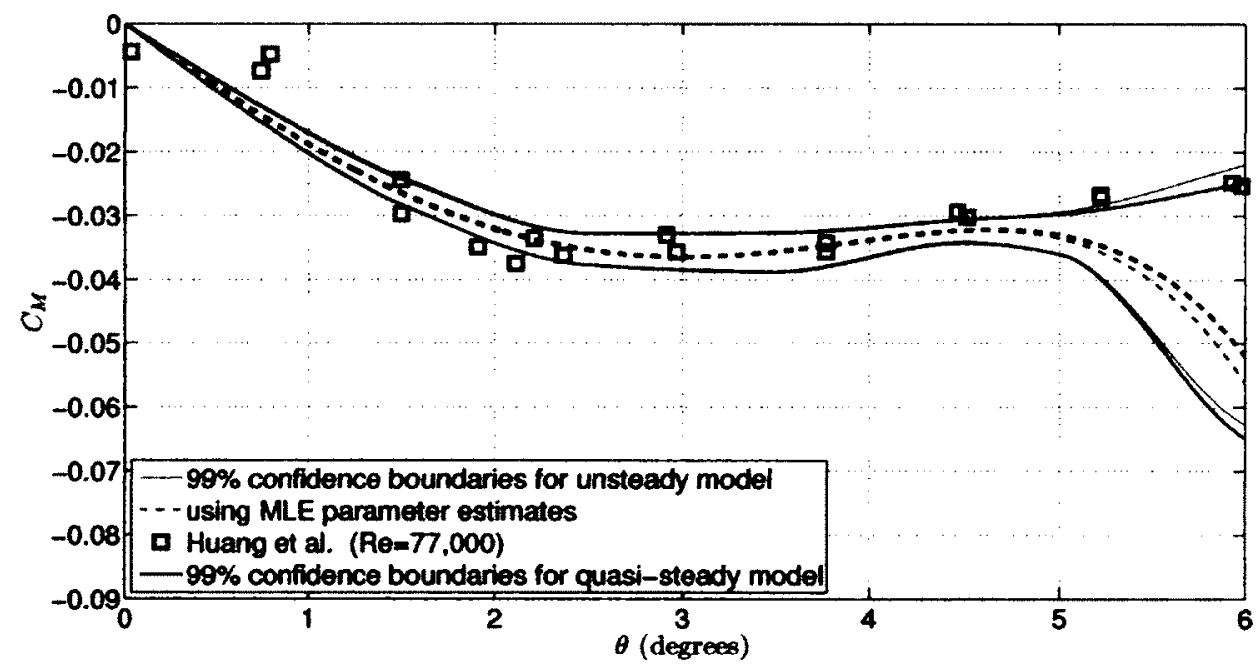

Figure 50: Comparison of the static aerodynamic moment coefficient for the quasisteady and the unsteady $5^{\text {th }}$ order generalized models for $\operatorname{Re}=85,000$

\begin{tabular}{|c|c|c|c|}
\hline Re values & $b$ in Eq. (103) & frequency of LCO $(\mathrm{Hz})$ & LCO amplitude (deg) \\
\hline 75,000 & $5.117342 \mathrm{E}+02$ & 3.02 & 5.05 \\
\hline 85,000 & $5.742059 \mathrm{E}+02$ & 3.22 & 5.27 \\
\hline 90,000 & $6.649934 \mathrm{E}+02$ & 3.35 & 4.90 \\
\hline 105,000 & $2.279164 \mathrm{E}+02$ & 3.95 & 4.13 \\
\hline
\end{tabular}

Table 16: MLE estimate of the coefficient of $M$ in Eq. (103) 


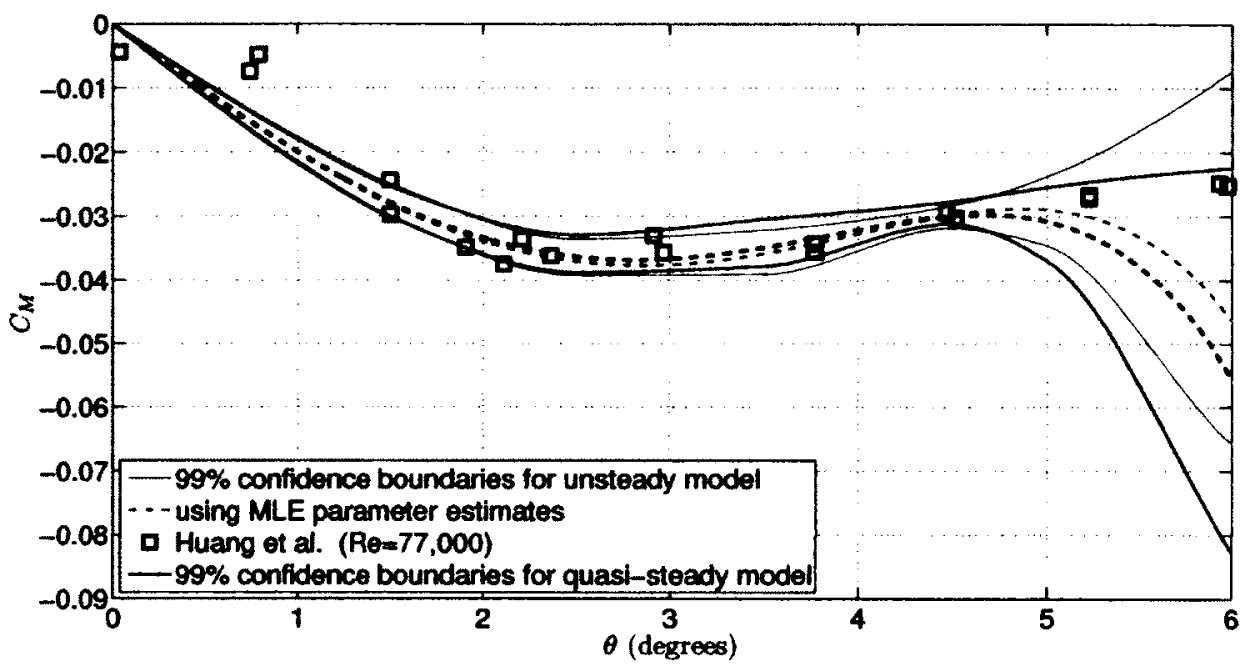

Figure 51: Comparison of the static aerodynamic moment coefficient for the quasisteady and the unsteady $5^{\text {th }}$ order generalized models for $\operatorname{Re}=90,000$

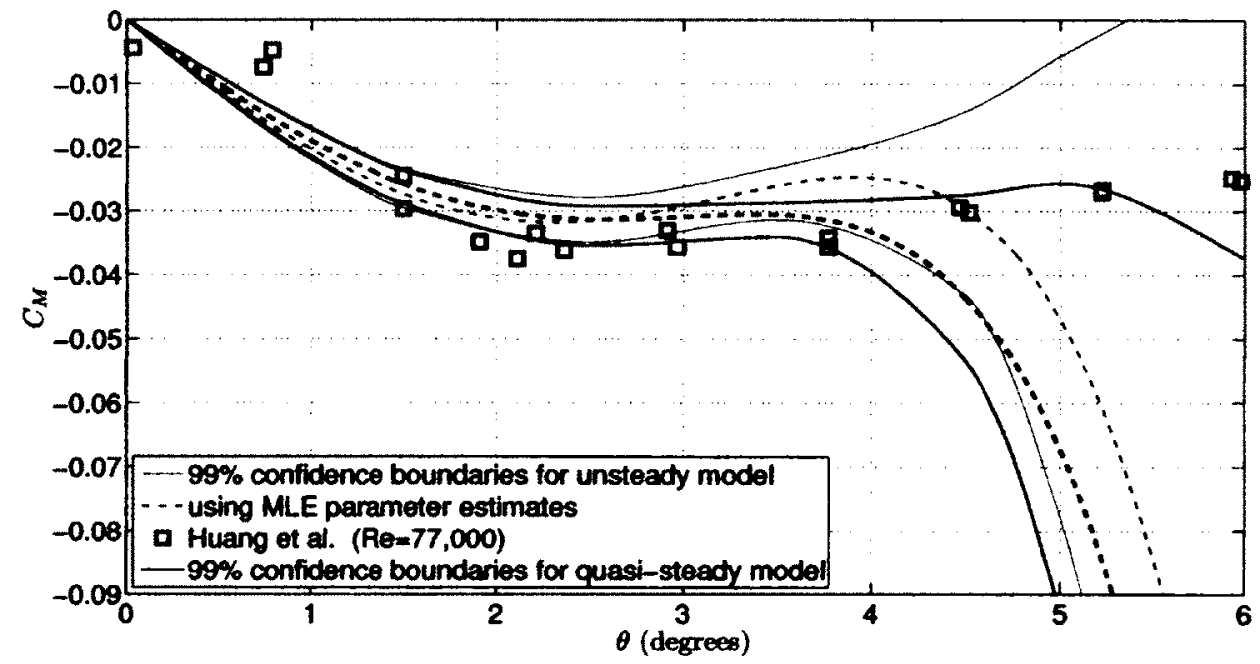

Figure 52: Comparison of the static aerodynamic moment coefficient for the quasisteady and the unsteady $5^{\text {th }}$ order generalized models for $\operatorname{Re}=105,000$ 


\section{Chapter 6}

\section{Conclusion and future work}

\subsection{Conclusion}

From Methodology point of view

- The Bayesian model selection technique involving the comparison of marginal likelihood estimated using the Chib-Jeliazkov method was presented. The HPC architecture was judiciously exploited to alleviate the dependence on asymptotic and often inaccurate model selection methods, thereby expediting the model selection process without degrading the accuracy level. The model selection results were reported for two numerical studies, which further bolstered the robustness of the model selection procedure. Finally, the model selection results for a nonlinear LCO response were used to make inferences about the system dynamics

- A general Bayesian parameter estimation technique adopted in this investigation leverages recent advances in the MCMC algorithm in conjunction with the high performance computing systems. In particular, the adaptive Metropolis-Hastings MCMC algorithm is implemented in parallel computers to 
perform efficient MCMC sampling for high dimensional posterior distributions. The comparison of the posterior pdfs with actual parameter values used in numerical studies highlights the efficiency of the algorithm to sample from complex posterior distributions.

\section{From application point of view}

- A nonlinear aeroelastic system consisting of a wing performing self-sustained oscillations in a wind-tunnel was successfully modeled using the proposed model selection and the parameter estimation techniques for four Reynolds number values. The nonlinear aerodynamic forces were identified by removing unnecessary terms from an initially proposed overly complex model. The static aerodynamic moment coefficient obtained using the final selected model and its posterior distribution was compared with the published data in the open literature. The comparisons were fairly conclusive.

- The unsteady effects were analyzed using an empirical extension of the Wagner's first order unsteady aerodynamics and the relevant results were reported.

\subsection{Future work}

Several extensions of this work are possible. Some of them are listed below:

- The model selection uncertainty is generally quite less for the "large data" type cases, as shown by Konishi [1]. However, it would be interesting to examine the model selection uncertainty for the marginal likelihood based methods and how it compares with those of information-theoretic methods. Also, the effect 
of measurement noise on the model selection uncertainty could be studied. The noise is expected to affect the model selection process significantly.

- The MCMC sampling algorithm can be further expedited through following two ways: 1) Use of Graphic Processing Units (GPUs) in conjunction with Central Preprocessing Units (CPUs) through hybrid computing, or 3) Use of a better optimization technique like simulated annealing or asynchronous parallel optimizations which can expedite the convergence of the MCMC chain, which can be time-consuming for certain prior and posterior distributions.

- The unsteady aerodynamics in the LCO oscillations can be further studied through a more elaborate model selection and parameter estimation procedure. 


\section{Appendix A}

\section{Sensitivity analysis : Chib-Jeliazkov method}

This appendix illustrates the sensitivity of the marginal likelihood estimate obtained using the Chib-Jeliazkov method [41] for different types of distributions.

\section{Bi-modal Gaussian distribution}

Fig. 53 and Fig. 54 show the marginal likelihood estimates for a bimodal gaussian distribution with a gaussian proposal of variance $w^{2}$. The MCMC samples in this case were generated from the expression:

$$
\mathrm{p}\left(\phi \mid d=\left\{d_{1}, d_{2}\right\}\right) \propto \frac{1}{\sigma_{1}} \exp \left\{-\frac{1}{2} \frac{\left(d_{1}-\phi\right)^{2}}{\sigma_{1}^{2}}\right\}+\frac{1}{\sigma_{2}} \exp \left\{-\frac{1}{2} \frac{\left(d_{2}-\phi\right)^{2}}{\sigma_{2}^{2}}\right\}
$$

The values used were: $d_{1}=3.0, d_{2}=-3.0, \sigma_{1}=1.0$ and $\sigma_{2}=1.0$. Note that the value of $\hat{\mathrm{p}}(\phi \mid d)$ shown in Fig. 53 and Fig. 54 are not to order.

\section{Log-normal distribution}

Fig. 55 and Fig. 56 show the marginal likelihood estimates for a bimodal gaussian 

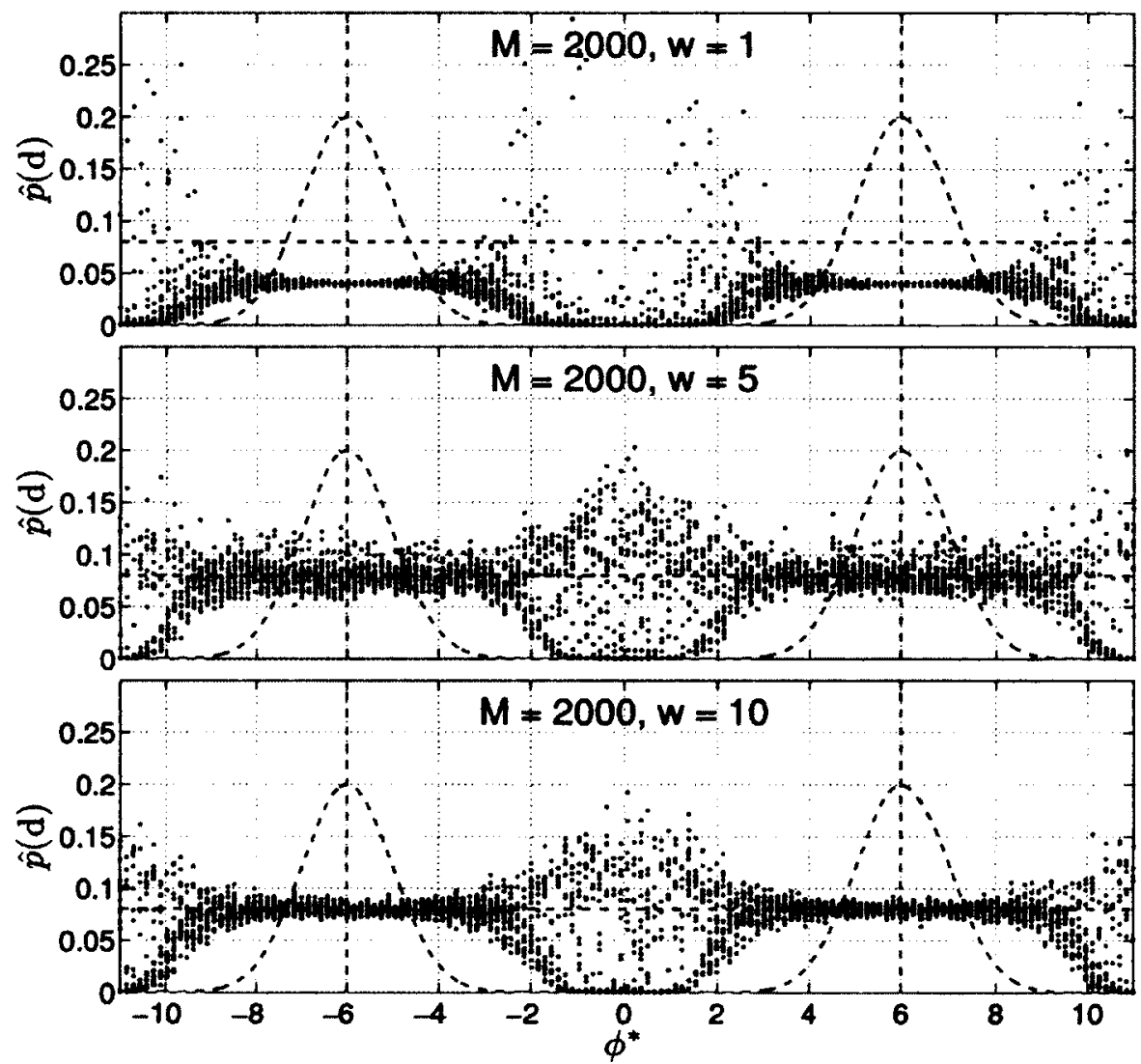

Figure 53: Marginal likelihood estimates for Bimodal gaussian distribution with modes at -6 and 6 

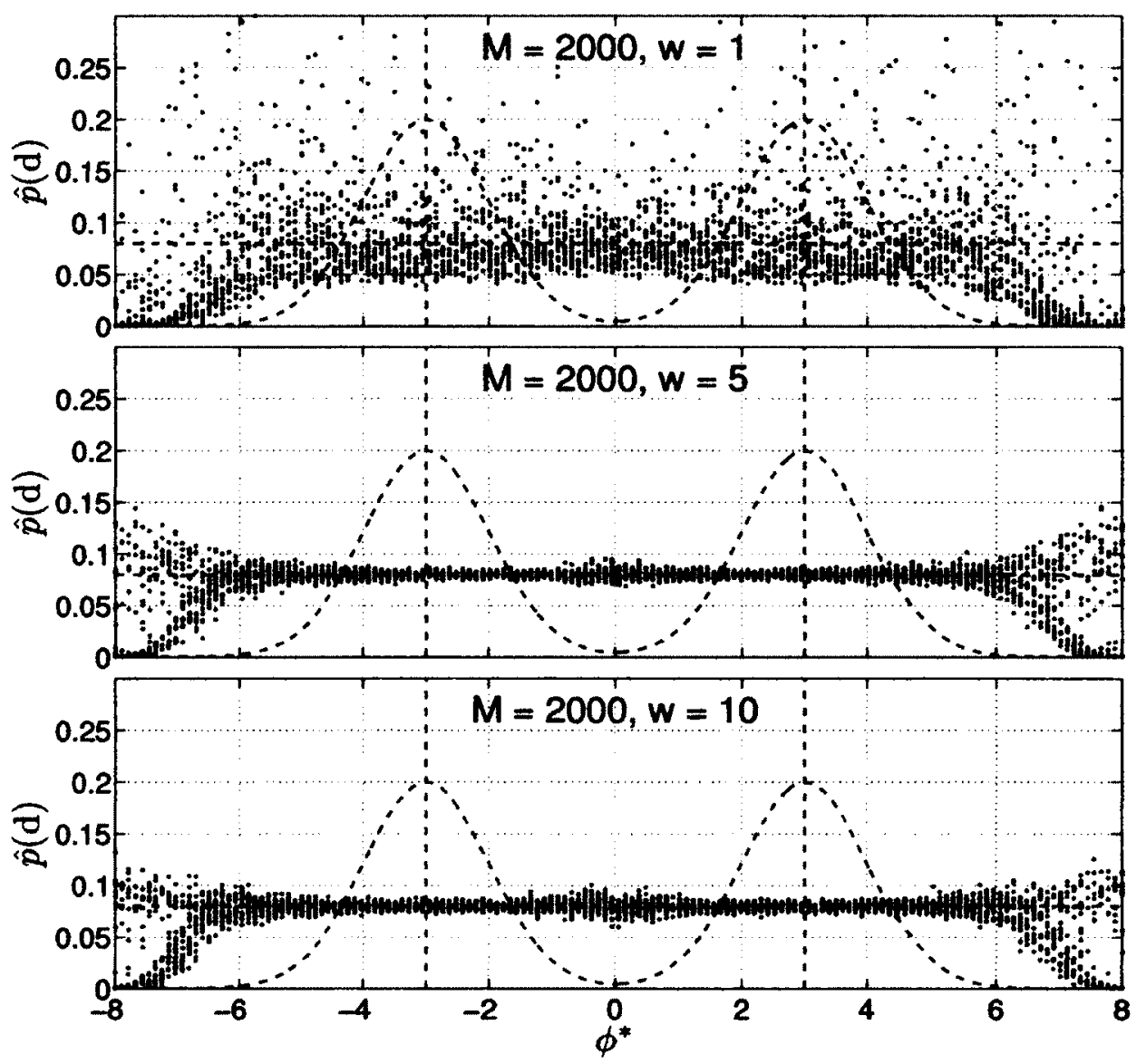

Figure 54: Marginal likelihood estimates for Bimodal gaussian distribution with modes at -3 and 3 
distribution with a gaussian proposal of variance $(w * \sigma)^{2}$. The MCMC samples in this case were generated from the expression:

$$
\mathrm{p}(\phi \mid d) \propto \frac{1}{\phi} \exp \left\{-\frac{1}{2} \frac{(d-\log \phi)^{2}}{\sigma^{2}}\right\}
$$

The values used were: $d=0.0$ and $\sigma=0.3$. Note that the value of $\hat{\mathrm{p}}(\phi \mid d)$ shown in Fig. 55 and Fig. 56 are not to order. The difference between Fig. 55 and Fig. 56 is with respect to the MCMC sampling algorithm. While $\phi$ is allowed to take negative values during the sampling for results in Fig. 55, only absolute values were considered during sampling for results in Fig. 56. It turns out that this condition actually helps improve the marginal likelihood estimates for higher proposal variances. 


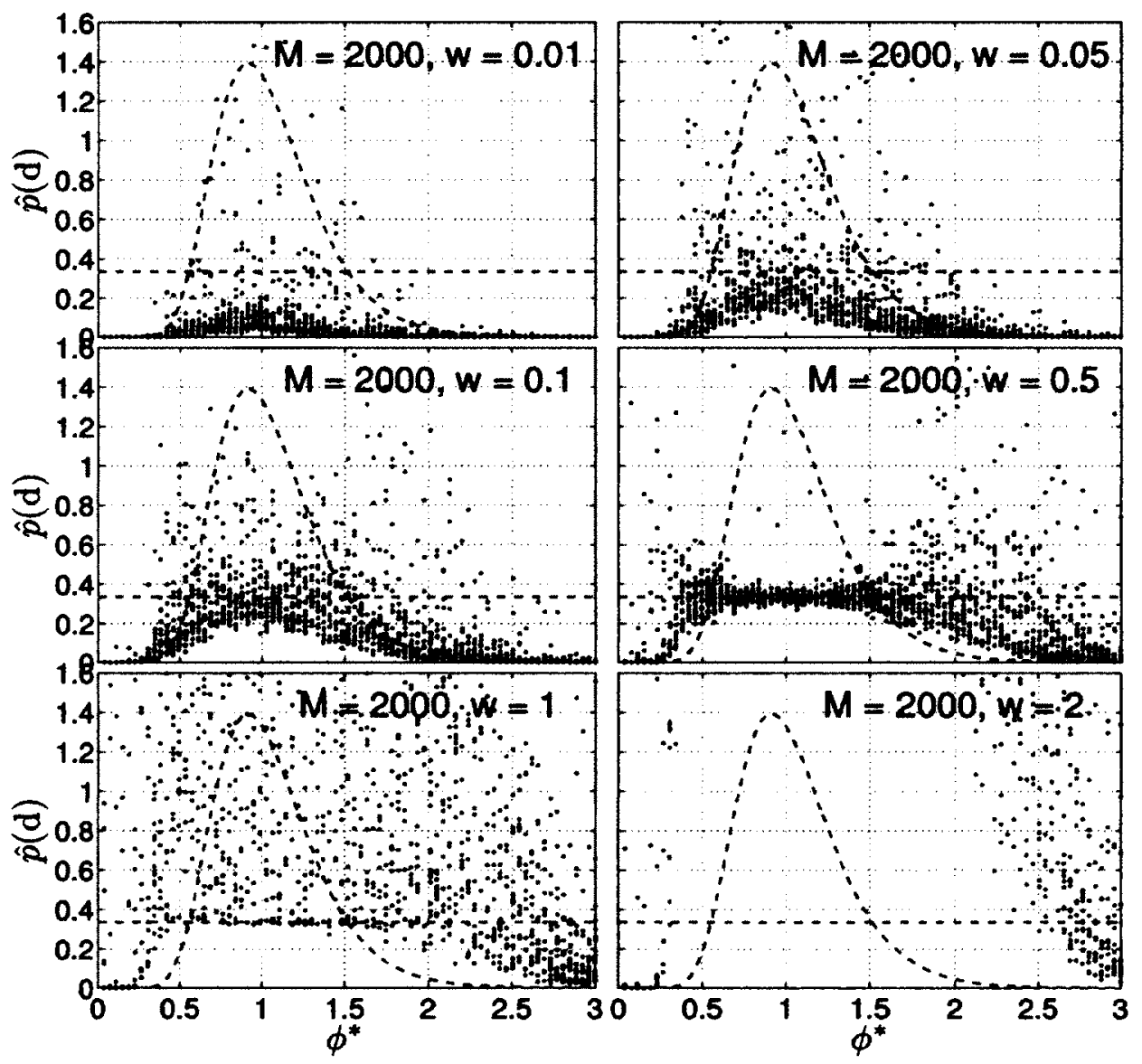

Figure 55: Marginal likelihood estimates for log-normal distribution without the positivity condition for MCMC samples 


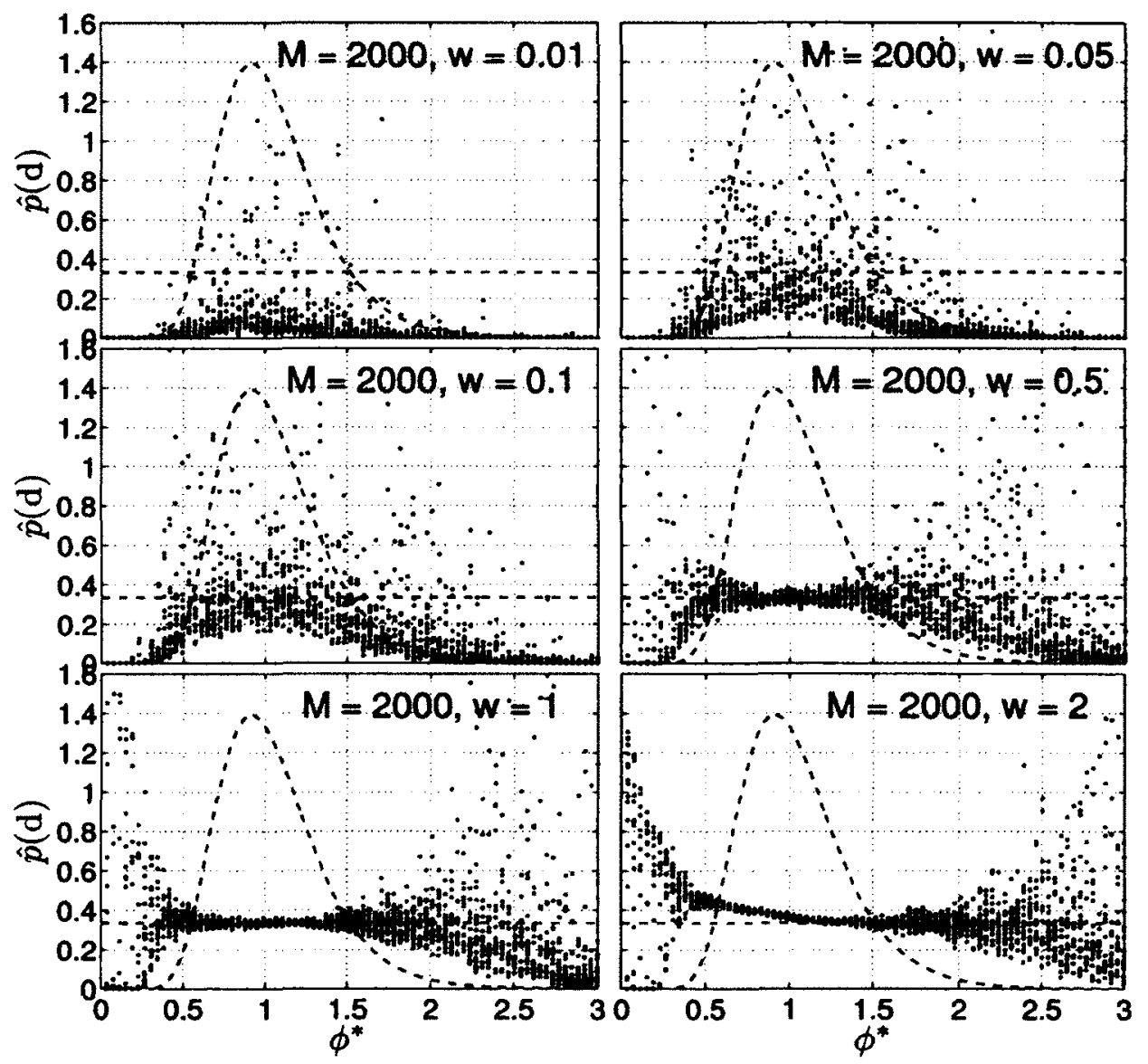

Figure 56: Marginal likelihood estimates for log-normal distribution with the positivity condition for MCMC samples 


\section{Appendix B}

\section{State space conversion through an example}

In this appendix, we will demonstrate the mathematical underpinnings involved in the numerical implementation of the model selection and parameter estimation equations derived in Chapter 3 and Chapter 4 through a example. We will simplify the equations by assuming a quasi-steady ODE model for the pure-pitch wing oscillations.

\section{State space conversion}

In this section, we will convert the ODE model structure into a discrete state-space representation of the form

$$
\begin{array}{lr}
\mathbf{x}_{k+1}=\mathbf{g}_{k}\left(\mathbf{x}_{k}, \mathbf{f}_{k}, \mathbf{q}_{k}\right) & \text { - Dynamic Model } \\
\mathbf{d}_{k}=\mathbf{h}_{k}\left(\mathbf{x}_{k}, \boldsymbol{\epsilon}_{k}\right) & \text { - Measurement Model. }
\end{array}
$$

For illustration purposes, we consider a wing with pure-pitch oscillations whose physics is represented by a stochastic, second-order ODE of the form

$$
\ddot{\theta}=f(\theta, \dot{\theta})+\sigma w_{1}(t),
$$


where $\theta$ is the pitch displacement, $f($.$) is some given scaler nonlinear function char-$ acterized by a static parameter vector $\phi, \sigma^{2}$ is the variance of the modelling error, and $w_{1}(t) \sim \mathcal{N}(0,1)$ is a white Gaussian noise process. The measurable quantity is stored in $\mathbf{d}_{1: n}=\left\{d_{1}, d_{2}, \ldots, d_{n}\right\}$ and is related to $\theta$ as

$$
d_{k}=\theta_{k}+\gamma w_{2}\left(t_{k}\right)
$$

where $\gamma^{2}$ is the variance of the measurement error and $w_{2}(t) \sim \mathcal{N}(0,1)$ is a white Gaussian noise process independent of $w_{1}(t)$.

To start with, we define the state vector $\mathbf{x}$ of the system as

$$
\mathbf{x}=\left\{\begin{array}{l}
x_{1} \\
x_{2}
\end{array}\right\}=\left\{\begin{array}{l}
\theta \\
\dot{\theta}
\end{array}\right\}
$$

Differentiating the state vector $\mathbf{x}$ and using Eq. (107), we get

$$
\dot{\mathbf{x}}=\left\{\begin{array}{c}
\dot{x}_{1} \\
\dot{x}_{2}
\end{array}\right\}=\left\{\begin{array}{c}
\dot{\theta} \\
\ddot{\theta}
\end{array}\right\}=\left\{\begin{array}{c}
x_{2} \\
f\left(x_{1}, x_{2}\right)
\end{array}\right\}+\left\{\begin{array}{c}
0 \\
\sigma w_{1}(t)
\end{array}\right\}
$$

We will represent Eq. (110) in the form

$$
\dot{\mathbf{x}}=\mathbf{f}^{\prime}(\mathbf{x})+L \mathbf{w}(t)
$$

where

$$
\mathbf{f}^{\prime}(\mathbf{x})=\left\{\begin{array}{c}
x_{2} \\
f\left(x_{1}, x_{2}\right)
\end{array}\right\}, L=\left[\begin{array}{ll}
0 & 0 \\
0 & \sigma
\end{array}\right] \text { and } \mathbf{w}(t) \sim \mathcal{N}\left(\left\{\begin{array}{l}
0 \\
0
\end{array}\right\},\left[\begin{array}{cc}
1 & 0 \\
0 & 1
\end{array}\right]\right)
$$


Next, Eq. (111) is discretized by integrating with respect to time from $t_{k}$ to $t_{k+1}$ as

$$
\int_{t_{k}}^{t_{k+1}} d \mathbf{x}=\int_{t_{k}}^{t_{k+1}} \mathbf{f}^{\prime}(\mathbf{x}) d t+\int_{t_{k}}^{t_{k+1}} L \mathbf{w}(t) d t
$$

Here, $t_{k+1}=t_{k}+\Delta t$ and $\Delta t$ assumed to be constant throughout the given data set d. We further assume that $\Delta t$ is very small such that function $\mathbf{f}^{\prime}(\mathbf{x})$ does not vary significantly between $t_{k}$ and $t_{k+1}$. In that case, we substitute $\mathbf{f}^{\prime}(\mathbf{x})$ by $\mathbf{f}^{\prime}\left(\mathbf{x}_{k}\right)$ for the time $t$ between $t_{k}$ and $t_{k+1}$ to simplify Eq. (113) as follows:

$$
\begin{aligned}
\mathbf{x}_{k+1}-\mathbf{x}_{k} & =\int_{t_{k}}^{t_{k+1}} \mathbf{f}^{\prime}\left(\mathbf{x}_{k}\right) d t+\int_{t_{k}}^{t_{k+1}} L \mathbf{w}(t) d t \\
& =\mathbf{x}_{k}+\mathbf{f}^{\prime}\left(\mathbf{x}_{k}\right) \Delta t+\int_{t_{k}}^{t_{k+1}} L \mathbf{w}(t) d t
\end{aligned}
$$

Since a stationary white noise process is defined as a derivative of a general Brownian motion [97], $\mathbf{w}(t)$ can be written as

$$
\mathbf{w}(t)=\frac{d \mathbf{B}(t)}{d t}
$$

where $\mathbf{B}(t)$ represents a two dimensional Weiner process [98]. Substituting Eq. (115) in Eq. (114b), we get

$$
\begin{aligned}
\mathbf{x}_{k+1} & =\mathbf{x}_{k}+\mathbf{f}^{\prime}\left(\mathbf{x}_{k}\right) \Delta t+L \int_{t_{k}}^{t_{k+1}} \frac{d \mathbf{B}(t)}{d t} d t \\
& =\mathbf{x}_{k}+\mathbf{f}^{\prime}\left(\mathbf{x}_{k}\right) \Delta t+L \int_{\mathbf{B}_{\left(t_{k}\right)}}^{\left.t_{k+1}\right)} d \mathbf{B}(t)
\end{aligned}
$$




$$
\begin{aligned}
& \left.=\mathbf{x}_{k}+\mathbf{f}^{\prime}\left(\mathbf{x}_{k}\right) \Delta t\right)+L\left(\mathbf{B}\left(t_{k+1}\right)-\mathbf{B}\left(t_{k}\right)\right) \\
& =\mathbf{x}_{k}+\mathbf{f}^{\prime}\left(\mathbf{x}_{k}\right) \Delta t+L\left(\mathbf{B}\left(t_{k}+\Delta t\right)-\mathbf{B}\left(t_{k}\right)\right)
\end{aligned}
$$

Using the independence property of the Brownian motion [97], we get

$$
\begin{aligned}
\mathbf{x}_{k+1} & =\mathbf{x}_{k}+\mathbf{f}^{\prime}\left(\mathbf{x}_{k}\right) \Delta t+L\left(\mathbf{B}\left(t_{k}\right)+\mathbf{B}(\Delta t)-\mathbf{B}\left(t_{k}\right)\right) \\
& =\mathbf{x}_{k}+\mathbf{f}^{\prime}\left(\mathbf{x}_{k}\right) \Delta t+L \mathbf{B}(\Delta t)
\end{aligned}
$$

Since $\Delta t$ is a constant, $\mathbf{B}(\Delta t)$, called the Weiner increment is a random vector having a gaussian pdf with the following properties [97]:

$$
E[\mathbf{B}(\Delta t)]=0 ; E\left[\mathbf{B}(\Delta t) \mathbf{B}^{T}(\Delta t)\right]=\Delta t \mathbf{I}_{2}
$$

where $I_{2}$ is a $2 \times 2$ Identity matrix. Using these properties, we can re-write Eq. (117b) as

$$
\mathbf{x}_{k+1}=\mathbf{f}^{\prime \prime}\left(\mathbf{x}_{k}\right)+\mathbf{q}_{k}
$$

where

$$
\mathbf{f}_{k}^{\prime \prime}\left(\mathbf{x}_{k}\right)=\mathbf{x}_{k}+\mathbf{f}^{\prime}\left(\mathbf{x}_{k}\right) \Delta t=\left\{\begin{array}{c}
x_{1, k}+x_{2, k} \Delta t \\
\left.x_{2, k}+f\left(x_{1, k}, x_{2, k}\right) \Delta t\right)
\end{array}\right\}
$$

and

$$
\mathbf{q}_{k} \sim \mathcal{N}\left(\left\{\begin{array}{l}
0 \\
0
\end{array}\right\},\left[\begin{array}{cc}
0 & 0 \\
0 & \Delta t \sigma^{2}
\end{array}\right]\right)
$$

Here, $x_{1, k}=x_{1}\left(t_{k}\right)$. Next, consider the measurement equation

$$
d_{k}=\theta_{k}+\gamma w_{2}\left(t_{k}\right)
$$


It can be re-written as

$$
d_{k}=\mathbf{H x}_{k}+\epsilon_{k}
$$

where

$$
\mathbf{H}=\left[\begin{array}{ll}
1 & 0
\end{array}\right] \text { and } \epsilon_{k} \sim \mathcal{N}\left(0, \gamma^{2}\right)
$$

This completes the state-space conversion process.

\section{EKF equations}

In this section, we will simplify the forecast and update equations of the EKF procedure by using the state-space form obtained in the first section. The EKF equations for a general representation of the form of Eq. (125) are derived in Chapter 4 and are listed below:

$$
\begin{array}{ll}
\text { Update : } & \mathbf{x}_{k}^{a}=\mathbf{x}_{k}^{f}+\mathbf{K}_{k}\left(\mathbf{d}_{k}-\mathbf{h}_{k}\left(\mathbf{x}_{k}^{f}, \overline{\boldsymbol{\epsilon}}_{k}\right)\right) \\
& \mathbf{P}_{k}^{a}=\left(\mathbf{I}-\mathbf{K}_{k} \mathbf{C}_{k}\right) \mathbf{P}_{k}^{f} \\
& \mathbf{K}_{k}=\mathbf{P}_{k}^{f} \mathbf{C}_{k}^{T}\left(\mathbf{C}_{k} \mathbf{P}_{k}^{f} \mathbf{C}_{k}^{T}+\mathbf{D}_{k} \Gamma_{k} \mathbf{D}_{k}^{T}\right)^{-1} \\
\text { Forecast }: & \mathbf{x}_{k+1}^{f}=\mathbf{g}_{k}\left(\mathbf{x}_{k}^{a}, \mathbf{f}_{k}, \overline{\mathbf{q}}_{k}\right) \\
& \mathbf{P}_{k+1}^{f}=\mathbf{A}_{k} \mathbf{P}_{k}^{a} \mathbf{A}_{k}^{T}+\mathbf{B}_{k} \mathbf{Q}_{k} \mathbf{B}_{k}^{T}
\end{array}
$$

For symbol notations, please refer to Chapter 4 . We simplify these EKF equations by comparing the general representations in Eq. (125) with the ones in Eq. (123) and Eq. (119) as follows:

$$
\begin{aligned}
\mathbf{x}_{k+1} & =\mathbf{g}_{k}\left(\mathbf{x}_{k}, \mathbf{f}_{k}, \mathbf{q}_{k}\right)=\mathbf{f}^{\prime \prime}\left(\mathbf{x}_{k}\right)+\mathbf{q}_{k}, \\
d_{k} & =\mathbf{h}_{k}\left(\mathbf{x}_{k}, \epsilon_{k}\right)=\mathbf{H} \mathbf{x}_{k}+\epsilon_{k} .
\end{aligned}
$$


Thereafter, we can deduce the Jacobian matrices involved in the EKF procedure as follows:

$$
\begin{aligned}
\mathbf{C}_{k} & =\left.\frac{\partial \mathbf{h}_{k}\left(\mathbf{x}_{k}, \epsilon_{k}\right)}{\partial \mathbf{x}_{k}}\right|_{\mathbf{x}_{k}=\mathbf{x}_{k}^{f}, \epsilon_{k}=\bar{\epsilon}_{k}} \\
& =\left.\frac{\partial\left(\mathbf{H} \mathbf{x}_{k}+\epsilon_{k}\right)}{\partial \mathbf{x}_{k}}\right|_{\mathbf{x}_{k}=\mathbf{x}_{k}^{f}, \epsilon_{k}=0} \\
& =\left.\frac{\partial\left(\mathbf{H} \mathbf{x}_{k}\right)}{\partial \mathbf{x}_{k}}\right|_{\mathbf{x}_{k}}=\mathbf{x}_{k}^{f}+0 \\
& =\mathbf{H}
\end{aligned}
$$

$$
\begin{aligned}
\mathbf{D}_{k} & =\left.\frac{\partial\left(\mathbf{h}_{k}\left(\mathbf{x}_{k}, \epsilon_{k}\right)\right.}{\partial \epsilon_{k}}\right|_{\mathbf{x}_{k}=\mathbf{x}_{k}^{f}, \epsilon_{k}=\bar{\epsilon}_{k}} \\
& =\left.\frac{\partial\left(\mathbf{H} \mathbf{x}_{k}+\epsilon_{k}\right)}{\partial \epsilon_{k}}\right|_{\mathbf{x}_{k}=\mathbf{x}_{k}^{f}, \epsilon_{k}=0} \\
& =0+\left.\frac{\partial\left(\epsilon_{k}\right)}{\partial \epsilon_{k}}\right|_{\epsilon_{k}=0}=0 \\
& =1
\end{aligned}
$$

$$
\begin{aligned}
\mathbf{A}_{k} & =\left.\frac{\partial \mathbf{g}_{k}\left(\mathbf{x}_{k}, \mathbf{f}_{k}, \mathbf{q}_{k}\right)}{\partial \mathbf{x}_{k}}\right|_{\mathbf{x}_{k}=\mathbf{x}_{k}^{a}, \mathbf{q}_{k}=\overline{\mathbf{q}}_{k}} \\
& =\left.\frac{\partial\left(\mathbf{f}^{\prime}\left(\mathbf{x}_{k}\right)+\mathbf{q}_{k}\right)}{\partial \mathbf{x}_{k}}\right|_{\mathbf{x}_{k}=\mathbf{x}_{k}^{a}, \mathbf{q}_{k}=0} \\
& =\left.\frac{\partial\left(\mathbf{f}^{\prime \prime}\left(\mathbf{x}_{k}\right)\right.}{\partial \mathbf{x}_{k}}\right|_{\mathbf{x}_{k}=\mathbf{x}_{k}^{a}+0}+0 \\
& =\left[\frac{\partial}{\partial \mathbf{x}_{k}}\left(\mathbf{x}_{k}+\mathbf{f}^{\prime}\left(\mathbf{x}_{k}\right) \Delta t\right)\right] \mathbf{x}_{k}=\mathbf{x}_{k}^{a} \\
& =\mathbf{I}_{2}+\Delta t \mathbf{G}_{k}^{a}
\end{aligned}
$$


where

$$
\begin{gathered}
\mathbf{G}_{k}^{a}=\left[\begin{array}{cc}
0 & 1 \\
\frac{\partial f\left(x_{1, k}, x_{2, k}\right)}{\partial x_{1, k}} & \frac{\partial f\left(x_{1, k}, x_{2, k}\right)}{\partial x_{2, k}}
\end{array}\right] \mid \mathbf{x}_{k}=\mathbf{x}_{k}^{a} \\
\mathbf{B}_{k}=\frac{\partial \mathbf{g}_{k}\left(\mathbf{x}_{k}, \mathbf{f}_{k}, \mathbf{q}_{k}\right)}{\partial \mathbf{q}_{k}} \mid \mathbf{x}_{k=\mathbf{x}_{k}^{a}, \mathbf{q}_{k}=\overline{\mathbf{q}}_{k}} \\
=\left.\frac{\partial\left(\mathbf{f}^{\prime \prime}\left(\mathbf{x}_{k}\right)+\mathbf{q}_{k}\right)}{\partial \mathbf{q}_{k}}\right|_{\mathbf{x}_{k}=\mathbf{x}_{k}^{a}, \mathbf{q}_{k}=0} \\
=0+\left.\frac{\partial\left(\mathbf{q}_{k}\right)}{\partial \mathbf{q}_{k}}\right|_{\mathbf{q}_{k}=0} \\
=\mathbf{I}_{2}
\end{gathered}
$$

Also, the error covariance matrices can be obtained using Eq. (121) and Eq. (124)as

$$
\Gamma_{k}=\gamma^{2} \text { and } \mathbf{Q}_{k}=\left[\begin{array}{cc}
0 & 0 \\
0 & \Delta t \sigma^{2}
\end{array}\right]
$$

Substituting matrices $\mathbf{A}_{k}, \mathbf{B}_{k}, \mathbf{C}_{k}, \mathbf{D}_{k}, \boldsymbol{\Gamma}_{k}$ and $\mathbf{Q}_{k}$ in the forecast equations of the EKF procedure, we obtain the Kalman gain matrix $\mathbf{K}_{k}$ as

$$
\begin{aligned}
\mathbf{K}_{k} & =\mathbf{P}_{k}^{f} \mathbf{C}_{k}^{T}\left(\mathbf{C}_{k} \mathbf{P}_{k}^{f} \mathbf{C}_{k}^{T}+\mathbf{D}_{k} \boldsymbol{\Gamma}_{k} \mathbf{D}_{k}^{T}\right)^{-1} \\
& =\mathbf{P}_{k}^{f} \mathbf{H}^{T}\left(\mathbf{H P}_{k}^{f} \mathbf{H}^{T}+\gamma^{2}\right)^{-1} \\
& =\mathbf{P}_{k}^{f} \mathbf{H}^{T} \frac{1}{\mathbf{P}_{11, k}^{f}+\gamma^{2}} \\
& =\left[\begin{array}{l}
\mathbf{P}_{11, k}^{f} \\
\mathbf{P}_{12, k}^{f}
\end{array}\right] \frac{1}{\mathbf{P}_{11, k}^{f}+\gamma^{2}}
\end{aligned}
$$

Using the Kalman gain matrix $\mathbf{K}_{k}$, we obtain the updated state mean $\mathbf{x}_{k}^{a}$ and updated 
state covariance matrix $\mathbf{P}_{k}^{a}$ as follows:

$$
\begin{aligned}
\mathbf{x}_{k}^{a} & =\mathbf{x}_{k}^{f}+\mathbf{K}_{k}\left(d_{k}-\mathbf{h}_{k}\left(\mathbf{x}_{k}^{f}, \bar{\epsilon}_{k}\right)\right) \\
& =\mathbf{x}_{k}^{f}+\mathbf{K}_{k}\left(d_{k}-\mathbf{H} \mathbf{x}_{k}^{f}+\bar{\epsilon}_{k}\right) \\
& =\left\{\begin{array}{l}
x_{1, k}^{f} \\
x_{2, k}^{f}
\end{array}\right\}+\left[\begin{array}{c}
\mathbf{P}_{11, k}^{f} \\
\mathbf{P}_{12, k}^{f}
\end{array}\right] \frac{1}{\mathbf{P}_{11, k}^{f}+\gamma^{2}}\left(d_{k}-\left[\begin{array}{ll}
1 & 0
\end{array}\right]\left\{\begin{array}{c}
x_{1, k}^{f} \\
x_{2, k}^{f}
\end{array}\right)\right) \\
& =\left\{\begin{array}{c}
x_{1, k}^{f} \\
x_{2, k}^{f}
\end{array}\right\}+\left[\begin{array}{c}
\mathbf{P}_{11, k}^{f} \\
\mathbf{P}_{12, k}^{f}
\end{array}\right] \frac{d_{k}-x_{1, k}^{f}}{\mathbf{P}_{11, k}^{f}+\gamma^{2}} \\
& \left\{\begin{array}{l}
\mathbf{x}_{1, k}^{a} \\
\mathbf{x}_{2, k}^{a}
\end{array}\right\}=\left\{\begin{array}{c}
x_{1, k}^{f}+\frac{\mathbf{P}_{11, k}^{f}}{\mathbf{P}_{11, k}^{f}+\gamma^{2}}\left(d_{k}-x_{1, k}^{f}\right) \\
x_{2, k}^{f}+\frac{\mathbf{P}_{12, k}^{f}}{\mathbf{P}_{11, k}^{f}+\gamma^{2}}\left(d_{k}-x_{1, k}^{f}\right)
\end{array}\right\}
\end{aligned}
$$

$$
\begin{aligned}
\mathbf{P}_{k}^{a} & =\left(\mathbf{I}_{2}-\mathbf{K}_{k} \mathbf{C}_{k}\right) \mathbf{P}_{k}^{f} \\
& =\mathbf{P}_{k}^{f}-\mathbf{K}_{k} \mathbf{H} \mathbf{P}_{k}^{f} \\
& =\mathbf{P}_{k}^{f}-\frac{1}{\mathbf{P}_{11, k}^{f}+\gamma^{2}} \mathbf{P}_{k}^{f} \mathbf{H}^{T} \mathbf{H} \mathbf{P}_{k}^{f} \\
& {\left[\begin{array}{cc}
\mathbf{P}_{11, k}^{a} & \mathbf{P}_{12, k}^{a} \\
\mathbf{P}_{12, k}^{a} & \mathbf{P}_{22, k}^{a}
\end{array}\right]=\frac{\gamma^{2}}{\mathbf{P}_{11, k}^{f}+\gamma^{2}}\left[\begin{array}{cc}
\mathbf{P}_{11, k}^{f} & \mathbf{P}_{12, k}^{f} \\
\mathbf{P}_{12, k}^{f} & \mathbf{P}_{22, k}^{f}-\frac{\operatorname{det}\left(\mathbf{P}_{k}^{f}\right)}{\gamma^{2}}
\end{array}\right] }
\end{aligned}
$$

The above equation implies that the elements of the update covariance matrix $\mathbf{P}_{k}^{a}$ are always smaller than the forecast covariance matrix $\mathbf{P}_{k}^{f}$ at the same time step, and thereby uncertainty reduces by assimilating observations. Next, we obtain the 
forecast state mean $\mathbf{x}_{k}^{f}$ and forecast state covariance matrix $\mathbf{P}_{k}^{f}$ as follows:

$$
\begin{gathered}
\mathbf{x}_{k+1}^{f}=\mathbf{g}_{k}\left(\mathbf{x}_{k}^{a}, \mathbf{f}_{k}, \overline{\mathbf{q}}_{k}\right) \\
=\mathbf{f}^{\prime}\left(\mathbf{x}_{k}^{a}\right)+\overline{\mathbf{q}}_{k} \\
\left.\qquad \begin{array}{l}
\mathbf{x}_{1, k+1}^{f} \\
\mathbf{x}_{2, k+1}^{f}
\end{array}\right\}=\left\{\begin{array}{c}
x_{1, k}^{a}+x_{2, k}^{a} \Delta t \\
x_{2, k}^{a}+f\left(x_{1, k}^{a}, x_{2, k}^{a}\right) \Delta t
\end{array}\right\} \\
\mathbf{P}_{k+1}^{f}=\mathbf{A}_{k} \mathbf{P}_{k}^{a} \mathbf{A}_{k}^{T}+\mathbf{B}_{k} \mathbf{Q}_{k} \mathbf{B}_{k}^{T} \\
\mathbf{P}_{k+1}^{f}=\left(\mathbf{I}_{2}+\Delta t \mathbf{G}_{k}^{a}\right) \mathbf{P}_{k}^{a}\left(\mathbf{I}_{2}+\Delta t \mathbf{G}_{k}^{a}\right)^{T}+\mathbf{I}_{2} \mathbf{Q}_{k} \mathbf{I}_{2}^{T} \\
\mathbf{P}_{k+1}^{f}=\left(\mathbf{I}_{2}+\Delta t \mathbf{G}_{k}^{a}\right) \mathbf{P}_{k}^{a}\left(\mathbf{I}_{2}+\Delta t\left(\mathbf{G}_{k}^{a}\right)^{T}\right)+\mathbf{Q}_{k} \\
\mathbf{P}_{k+1}^{f}=\mathbf{I}_{2} \mathbf{P}_{k}^{a} \mathbf{I}_{2}+\Delta t\left(\mathbf{P}_{k}^{a}\left(\mathbf{G}_{k}^{a}\right)^{T}+\mathbf{G}_{k}^{a} \mathbf{P}_{k}^{a}\right)+\Delta t^{2}\left(\mathbf{G}_{k}^{a} \mathbf{P}_{k}^{a}\left(\mathbf{G}_{k}^{a}\right)^{T}\right)+\mathbf{Q}_{k} \\
=\mathbf{P}_{k}^{a}+\Delta t \mathbf{R}_{k}^{a}+\Delta t^{2} \mathbf{S}_{k}^{a}+\mathbf{Q}_{k}
\end{gathered}
$$

where

$$
\mathbf{R}_{k}^{a}=\mathbf{P}_{k}^{a}\left(\mathbf{G}_{k}^{a}\right)^{T}+\mathbf{G}_{k}^{a} \mathbf{P}_{k}^{a} \text { and }=\mathbf{S}_{k}^{a}=\mathbf{G}_{k}^{a} \mathbf{P}_{k}^{a}\left(\mathbf{G}_{k}^{a}\right)^{T}
$$

\section{Posterior distribution}

In this section, we will simplify the Bayesian inference expression for obtaining the posterior distribution by using the simplified EKF equations obtained in the previous section. The general expression for obtaining the posterior distribution is derived in Chapter 4 and is given by

$$
\mathrm{p}\left(\phi \mid \mathbf{d}_{1: n}\right) \propto \mathrm{p}(\phi) \prod_{k=1}^{n} \mathcal{N}\left(\mathbf{d}_{k} \mid \mathbf{h}_{k}\left(\mathbf{x}_{k}^{f}, \bar{\epsilon}_{k}\right), \mathbf{C}_{k} \mathbf{P}_{k}^{f} \mathbf{C}_{k}^{T}+\mathbf{D}_{k} \boldsymbol{\Gamma}_{k} \mathbf{D}_{k}^{T}\right)
$$


For flat priors $(p(\phi) \propto 1)$, the general expression in Eq. (139) for the posterior distribution is reduced as follows:

$$
\begin{aligned}
\mathrm{p}(\phi \mid \mathbf{d}) & \propto \mathrm{p}(\phi) \prod_{k=1}^{n} \mathcal{N}\left(d_{k} \mid \mathbf{h}_{k}\left(\mathbf{x}_{k}^{f}, \bar{\epsilon}_{k}\right), \mathbf{C}_{k} \mathbf{P}_{k}^{f} \mathbf{C}_{k}^{T}+\mathbf{D}_{k} \Gamma_{k} \mathbf{D}_{k}^{T}\right) \\
& \propto \prod_{k=1}^{n} \mathcal{N}\left(d_{k} \mid \mathbf{H} \mathbf{x}_{k}^{f}+\bar{\epsilon}_{k}, \mathbf{H P}_{k}^{f} \mathbf{H}^{T}+\gamma^{2}\right) \\
& \propto \prod_{k=1}^{n} \mathcal{N}\left(d_{k} \mid \mathbf{x}_{11, k}^{f}, \mathbf{P}_{11, k}^{f}+\gamma^{2}\right) \\
& \propto \prod_{k=1}^{n} \frac{1}{\mathbf{P}_{11, k}^{f}+\gamma^{2}} \exp \left\{-\frac{1}{2} \frac{\left(d_{k}-\mathbf{x}_{11, k}^{f}\right)^{2}}{\mathbf{P}_{11, k}^{f}+\gamma^{2}}\right\}
\end{aligned}
$$




\section{Appendix C}

\section{Gaussian identities}

Linear operations [99]

If $\mathrm{p}(\mathbf{x})=\mathcal{N}(\boldsymbol{\mu}, \boldsymbol{\Sigma})$, then

$$
\begin{aligned}
\mathrm{p}(\mathbf{A x}+\mathbf{b}) & =\mathcal{N}\left(\mathbf{A} \boldsymbol{\mu}+\mathbf{b}, \mathbf{A} \boldsymbol{\Sigma} \mathbf{A}^{T}\right) \\
\mathrm{p}\left(\boldsymbol{\Sigma}^{-1 / 2}(\mathbf{x}-\boldsymbol{\mu})\right) & =\mathcal{N}(0, \mathbf{I})
\end{aligned}
$$

Marginal and conditional distributions [99]

If $\mathbf{x}$ and $\mathbf{y}$ are two random vectors with joint distribution

$$
\mathrm{p}(\mathbf{x}, \mathbf{y})=\mathcal{N}\left(\left[\begin{array}{l}
\mathbf{x} \\
\mathbf{y}
\end{array}\right] \mid\left(\begin{array}{l}
\mu_{x} \\
\mu_{y}
\end{array}\right),\left(\begin{array}{cc}
\Sigma_{x} & \Sigma_{x y} \\
\Sigma_{x y}^{T} & \Sigma_{y}
\end{array}\right)\right)
$$

then 


$$
\begin{aligned}
\mathrm{p}(\mathbf{x}) & =\mathcal{N}\left(\boldsymbol{\mu}_{x}, \boldsymbol{\Sigma}_{x}\right) \\
\mathrm{p}(\mathbf{y}) & =\mathcal{N}\left(\boldsymbol{\mu}_{y}, \boldsymbol{\Sigma}_{y}\right) \\
\mathrm{p}(\mathbf{x} \mid \mathbf{y}) & =\mathcal{N}\left(\boldsymbol{\mu}_{x}+\boldsymbol{\Sigma}_{x y} \boldsymbol{\Sigma}_{y}^{-1}\left(\mathbf{y}-\boldsymbol{\mu}_{y}\right), \boldsymbol{\Sigma}_{x}-\boldsymbol{\Sigma}_{x y} \boldsymbol{\Sigma}_{y}^{-1} \boldsymbol{\Sigma}_{x y}^{T}\right) \\
\mathrm{p}(\mathbf{y} \mid \mathbf{x}) & =\mathcal{N}\left(\boldsymbol{\mu}_{y}+\boldsymbol{\Sigma}_{x y}^{T} \boldsymbol{\Sigma}_{x}^{-1}\left(\mathbf{x}-\boldsymbol{\mu}_{x}\right), \boldsymbol{\Sigma}_{y}-\boldsymbol{\Sigma}_{x y}^{T} \boldsymbol{\Sigma}_{x}^{-1} \boldsymbol{\Sigma}_{x y}\right)
\end{aligned}
$$

\section{Multiplication of two gaussian distributions [99]}

If $\mathbf{x}$ and $\mathbf{y}$ are two random vectors with $\mathrm{p}(\mathbf{x})=\mathcal{N}\left(\boldsymbol{\mu}_{x}, \boldsymbol{\Sigma}_{x}\right)$ and $\mathrm{p}(\mathbf{y})=\mathcal{N}\left(\boldsymbol{\mu}_{y}, \boldsymbol{\Sigma}_{y}\right)$ and

$$
\mathbf{z} \propto \mathbf{x} \times \mathbf{y}
$$

then $\mathrm{p}(\mathbf{z})=\mathcal{N}\left(\boldsymbol{\mu}_{z}, \boldsymbol{\Sigma}_{z}\right)$ where

$$
\begin{aligned}
& \boldsymbol{\Sigma}_{z}=\left(\boldsymbol{\Sigma}_{x}^{-1}+\boldsymbol{\Sigma}_{y}^{-1}\right)^{-1} \\
& \boldsymbol{\mu}_{z}=\boldsymbol{\Sigma}_{z} \boldsymbol{\Sigma}_{x}^{-1} \boldsymbol{\mu}_{x}+\boldsymbol{\Sigma}_{z} \boldsymbol{\Sigma}_{y}^{-1} \boldsymbol{\mu}_{y}
\end{aligned}
$$




\section{Bibliography}

[1] S. Konishi and G. Kitagawa. Information Criteria and Statistical Modeling (Springer Series in Statistics). Springer. ISBN 0387718869 (2007).

[2] K. P. Burnham and D. R. Anderson. Model selection and inference: a practical information-theoretic approach. Springer-Verlag. ISBN 0387985042 (1998).

[3] D. Poirel and W. Yuan. "Aerodynamics of laminar separation flutter at a transitional Reynolds number." Journal of Fluids and Structures 26(7-8), 1174-1194. ISSN 08899746 (2010).

[4] D. Poirel, Y. Harris, and a. Benaissa. "Self-sustained aeroelastic oscillations of a NACA0012 airfoil at low-to-moderate Reynolds numbers." Journal of Fluids and Structures 24(5), 700-719. ISSN 08899746 (2008).

[5] M. Khalil, P. Poirel, and A. Sarkar. "Probabilistic Parameter Estimation of a Fluttering Aeroelastic System in the Transitional Reynolds Number Regime." Joumal of Sound and Vibration (under review) .

[6] H. Jeffreys. Theory of probability. Oxford University Press. ISBN 0198503687, $9780198503682(1939)$.

[7] W. H. Jefferys and J. O. Berger. "Ockham's Razor and Bayesian Analysis." American Scientist 80(1), 64-72 (1992). 
[8] I. Guyon and G. Cawley. "Model Selection : Beyond the Bayesian / Frequentist Divide." Journal of Machine Learning Research 11, 61-87. ISSN 15324435 (2010).

[9] H. Walach. "Ockham's razor." In "Wiley Interdisciplinary Reviews Computational Statistics," volume 2, pages n/a-n/a. Sage Publications (2007).

[10] G. E. P. Box and G. M. Jenkins. Time Series Analysis: Forecasting and Control, volume Third of Holden-Day Series in Time Series Analysis. Prentice Hall. ISBN 0130607746 (1994).

[11] J. O. Berger, L. R. Pericchi, J. K. Ghosh, T. Samanta, F. D. Santis, J. O. Berger, and L. R. Pericchi. "Objective Bayesian Methods for Model Selection: Introduction and Comparison." Lecture NotesMonograph Series 38(2001), 135 207. ISSN $07492170(2001)$.

[12] R. E. Kass and A. E. Raftery. "Bayes Factors." Journal of the American Statistical Association 90(430), pp. 773-795. ISSN 01621459 (1995).

[13] J. B. Kadane and N. A. Lazar. "Methods and Criteria for Model Selection." Journal of the American Statistical Association 99(465), 279-290. ISSN 01621459 (2004).

[14] W. Zucchini. "An Introduction to Model Selection." Journal of Mathematical Psychology 44(1), 41-61. ISSN 00222496 (2000).

[15] C. P. Robert, N. Chopin, and J. Rousseau. "Harold Jeffreys Theory of Probability Revisited." Statistical Science 24(2), 141-172. ISSN 08834237 (2009).

[16] A. Gelman. "Bayes, Jeffreys, Prior Distributions and the Philosophy of Statistics." Statistical Science 24(2), 141-172 (2010). 
[17] S. Gull. "Bayesian inductive inference and maximum entropy." In G. Erickson and C. R. Smith, editors, "Maximum entropy and Bayesian methods in science and engineering," volume 1, pages 53-74. Kluwer Academic Publishers (1988).

[18] D. J. C. MacKay. "Bayesian Interpolation." Neural Computation 4(3), 415-447. ISSN 08997667 (1992).

[19] D. S. Sivia and J. Skilling. Data Analysis: A Bayesian Tutorial, volume Second Edi of Oxford science publications. Oxford University Press, USA. ISBN $0198568320(2006)$.

[20] W. Vanpaemel. "Prior sensitivity in theory testing: An apologia for the Bayes factor." Journal of Mathematical Psychology 54(6), 491-498. ISSN 00222496 (2010).

[21] S. Sinharay and H. S. Stern. "On the sensitivity of Bayes factors to the prior distributions. (General).(Statistical Data Included)." American Statistician 56(3), 0 . ISSN 00031305 (2002).

[22] A. L. Rukhin. "Influence of the prior distribution on the risk of the Bayes rule." Journal of Theoretical Probability 6(1), 71-87. ISSN 08949840 (1993).

[23] C. Liu and M. Aitkin. "Bayes factors: Prior sensitivity and model generalizability." Journal of Mathematical Psychology 52(6), 362-375. ISSN 00222496 (2008).

[24] S. Sinharay and H. S. Stern. "On the Sensitivity of Bayes Factors to the Prior Distributions." American Statistician 56(3), 196-201. ISSN 00031305 (2002).

[25] D. V. Lindley. "A Statistical Paradox." Biometrika 44(1/2), 187-192. ISSN 00063444 (1957). 
[26] H. van Strachan, R.W. and Dijk. "Improper priors with well defined Bayes Factors." Technical report, Econometric Institute Report (2004).

[27] R. W. Strachan and H. K. van Dijk. "Divergent Priors and Well Behaved Bayes Factors." SSRN Electronic Journal ISSN 1556-5068 (2011).

[28] J. O. Berger and L. R. Pericchi. "The Intrinsic Bayes Factor for Model Selection and Prediction." Journal of the American Statistical Association 91(433), 109. ISSN 01621459 (1996).

[29] A. Rodriguez and L. R. Pericchi. "Intrinsic Bayes Factors for Dynamic Linear Models ." ISBA and Eurostat (2001).

[30] J. O. Berger and J. Mortera. "Default Bayes Factors for Nonnested Hypothesis Testing." Joumal of the American Statistical Association 94(446), 542-554. ISSN 01621459 (1999).

[31] A. O'Hagan. "Fractional Bayes Factors for Model Comparison." Journal of the Royal Statistical Society Series B Methodological 57(1), 99-138. ISSN 00359246 (1995).

[32] M. Cavalcante. "Fractional Bayes Factor for Gaussian Linear State Space Models." Technical Report 1, Universidade do Amazones ICE (2001).

[33] M. Aitkin. "Posterior Bayes factor analysis for an exponential regression model." Statistics and Computing 3(1), 17-22. ISSN 09603174 (1993).

[34] J. O. Berger, L. R. Pericchi, J. K. Ghosh, T. Samanta, F. D. Santis, J. O. Berger, and L. R. Pericchi. "Objective Bayesian Methods for Model Selection: Introduction and Comparison." Lecture NotesMonograph Series 38(2001), 135-207. ISSN 07492170 (2001). 
[35] C. H. Carlin and B. P. "MCMC Methods for Computing Bayes Factors : A Comparative Review." Journal of the American Statistical Association 96(612), $1122-1132(2000)$.

[36] P. J. Green and D. I. Hastie. "Reversible jump MCMC." Bioinformatics 24(13), 1-26. ISSN 13674811 (2009).

[37] L. Wasserman. "Bayesian Model Selection and Model Averaging." Journal of Mathematical Psychology 44(1), 92-107. ISSN 00222496 (2000).

[38] W. Penny, J. Mattout, and N. Trujillo-Barreto. "Bayesian model selection and averaging." In K. Friston, J. Ashburner, S. Kiebel, T. Nichols, and W. Penny, editors, "Journal of Mathematical Psychology," volume 44, chapter 35, pages 454-467. Elsevier (2007).

[39] J. Montgomery and B. Nyhan. "Bayesian Model Averaging." Journal of Econometrics 18(2), 245-270. ISSN 10471987 (2010).

[40] S. Chib. "Marginal Likelihood from the Gibbs Output." Journal of the American Statistical Association 90(432), 1313-1321. ISSN 01621459 (1995).

[41] S. Chib and I. Jeliazkov. "Marginal Likelihood From the Metropolis-Hastings Output." Journal of the American Statistical Association 96(453), 270-281. ISSN 01621459 (2001).

[42] H. Akaike. "Information theory as an extension of the maximum likelihood principle." In B. N. Petrov and F. Csaki, editors, "Second International Symposium on Information Theory," volume 19, pages 267-281. Akademiai Kiado (1973).

[43] G. Schwarz. "Estimating the dimension of a model." Annals of Statistics 6(2), 461-464. ISSN 00905364 (1978). 
[44] R. E. K. Raftery and A. E. "Bayes factors and model uncertainty." Technical Report 254, DEPARTMENT OF STATISTICS, UNIVERSITY OF WASHINGTON (1993).

[45] K. P. Burnham. "Multimodel Inference: Understanding AIC and BIC in Model Selection." Sociological Methods Research 33(2), 261-304. ISSN 00491241 (2004).

[46] A. B. Koehler and E. S. Murphree. "A comparison of the Akaike and Schwarz criteria for selecting model order." Applied Statistics 37(2), 187-195. ISSN $00359254(1988)$.

[47] A. F. M. Smith and D. J. Spiegelhalter. "Bayes Factors and Choice Criteria for Linear Models." Journal of the Royal Statistical Society B 42(2), 213-220. ISSN $00359246(1980)$

[48] H. Bozdogan. "Akaike's Information Criterion and Recent Developments in Information Complexity." Journal of mathematical psychology 44(1),62-91. ISSN 0022-2496 (2000).

[49] W. R. Lionheart. "Inverse problems in industry." Inverse Problems (February) (2008).

[50] S. N. Evans and P. B. Stark. "Inverse problems as statistics." Inverse Problems 18(4), R55-R97. ISSN 02665611 (2002).

[51] M. Bonnet and A. Constantinescu. "Inverse problems in elasticity." Inverse Problems 21(2), R1-R50. ISSN 02665611 (2005).

[52] E.-J. Wagenmakers, M. Lee, T. Lodewyckx, and G. J. Iverson. "Bayesian versus frequentist inference." Bayesian evaluation of informative hypotheses pages 181207 (2008). 
[53] B. Efron. "THE BAYESIAN-FREQUENTIST CONTROVERSY." Journal Of Marriage And The Family 56, 1-13 (2005).

[54] F. J. Samaniego. A Comparison of the Bayesian and Frequentist Approaches to Estimation. Springer Series in Statistics. Springer. ISBN 9781441959409 (2010).

[55] I. Myung. "Tutorial on maximum likelihood estimation." Journal of Mathematical Psychology 47(1), 90-100. ISSN 00222496 (2003).

[56] A. O'Hagan and J. Forster. "Bayesian inference." Technical report (1994).

[57] E. Somersalo and J. Kaipio. Statistical and Computational Inverse Problems. Springer, 1 edition. ISBN 0387220739 (2004).

[58] S. Tan, C. Fox, and G. Nicholas. "Physics 707 (Inverse Problems Course) Lecture Notes." (2009).

[59] J. L. Beck and L. S. Katafygiotis. "Updating Models and Their Uncertainties. I: Bayesian Statistical Framework." Journal of Engineering Mechanics 124(4), 455-461. ISSN 07339399 (1998).

[60] J. L. Beck and K.-v. Yuen. "Model selection using response measurements: Bayesian probabilistic approach." Journal of Engineering Mechanics 41, 192203. ISSN 08873585 (2004).

[61] J. Beck and K. Woodbury. "Inverse problems and parameter estimation : integration of measurements and analysis." Measurement Science and Technology 9(1), 839-847 (1998).

[62] A. Miller. Subset Selection in Regression, volume 9 of C6 $3 H / C R C$ Monographs on Statistics \& Applied Probability. Chapman \& Hall. ISBN 1584881712 (2002). 
[63] A. J. Miller. "Selection of subsets of regression variables." Journal Of The Royal Statistical Society Series A General 147(3), 389-425. ISSN 00359238 (1984).

[64] A. A. A. El-Sallam, S. Kayhan, and A. M. Zoubir. "Bootstrap and backward elimination based approaches for model selection." In S. Loncaric, A. Neri, and H. Babic, editors, "3rd International Symposium on Image and Signal Processing and Analysis 2003 ISPA 2003 Proceedings of the," volume 1, pages 152-157. Ieee. ISBN 953184061X. ISSN 13301012 (2003).

[65] R. Mundry and C. L. Nunn. "Stepwise model fitting and statistical inference: turning noise into signal pollution." The American naturalist 173(1), 119-23. ISSN 15375323 (2009).

[66] S. Derksen and H. J. Keselman. "Backward, forward and stepwise automated subset selection algorithms: frequency of obtaining authentic and noise variables." British Journal of Mathematical and Statistical Psychology 45(2), 265282. ISSN 00071102 (1992).

[67] N. Friel and A. N. Pettitt. "Marginal likelihood estimation via power posteriors." Journal of the Royal Statistical Society - Series B: Statistical Methodology 70(3), 589-607. ISSN 13697412 (2008).

[68] S. Chib and E. Greenberg. "Understanding the Metropolis-Hastings Algorithm." American Statistician 49(4), 327-335. ISSN 00031305 (1995).

[69] W. Gilks, D. Spiegelhalter, and S. Richardson. Markov chain Monte Carlo in practice: Interdisciplinary Statistics. Chapman \& Hall, London, 1 edition. ISBN 0412055511 (1996).

[70] W. Link and M. Eaton. "On thinning of chains in MCMC." Methods in Ecology and Evolution 3(1), 112-115. ISSN 2041210X (2012). 
[71] R. Rojas. "The Kalman Filter." The Mathematical Intelligencer 1(2), 1-7 (1978).

[72] G. O. Roberts and J. S. Rosenthal. "Optimal Scaling for Various MetropolisHastings Algorithms." Statistical Science 16(4), pp. 351-367 (2001).

[73] G. O. Roberts, A. Gelman, and W. R. Gilks. "Weak convergence and optimal scaling of random walk Metropolis algorithms." The Annals of Applied Probability 7(1), 110-120. ISSN 10505164 (1997).

[74] H. Haario, M. Laine, A. Mira, and E. Saksman. "DRAM: efficient adaptive MCMC." Statistics and Computing 16(4), 339-354. ISSN 0960-3174 (2006).

[75] M. B. Thompson. "A Comparison of Methods for Computing Autocorrelation Time." Technical Report 1007, University of Toronto Statistics Department Technical Report (2010).

[76] H. Haario and E. Saksman. "Adaptive proposal distribution for random walk Metropolis algorithm." Computational Statistics 4(Yliopistonkatu 5), 1-32 (1999).

[77] H. Haario, E. Saksman, and J. Tamminen. "An adaptive Metropolis algorithm." Bernoulli pages 223-242 (2001).

[78] T. L. Graves. "Automatic Step Size Selection in Random Walk Metropolis Algorithms." Philosophy page 13 (2011).

[79] A. H. Jazwinski. Stochastic Processes and Filtering Theory. Academic Press. ISBN 0123815509 (1970).

[80] G. Chui, C. K. and Chen. Kalman filtering with real-time applications. SpringerVerlag New York, Inc., New York, NY, USA. ISBN 0-387-18385-7 (1987). 
[81] M. S. Grewal and A. P. Andrews. Kalman filtering: theory and practice using $M A T L A B$, volume 5. John Wiley \& Sons, Inc. ISBN 0471392545 (2001).

[82] J. S. Liu. Monte Carlo strategies in scientific computing. Springer. ISBN $0387952306(2001)$.

[83] N. Metropolis, A. Rosenbluth, M. Rosenbluth, A. Teller, and E. Teller. "Equation-of-State Calculations by Fast Computing Machines." Joumal of Chemical Physics 21(6), 1087-1092 (1953).

[84] W. K. Hastings. "Monte Carlo sampling methods using Markov chains and their applications." Biometrika 57(1), 97-109. ISSN 00063444 (1970).

[85] G. Poirel, D. and Métivier, V. and Dumas. "Computational aeroelastic simulations of self-sustained pitch oscillations of a NACA0012 at transitional Reynolds numbers." Journal of Fluids and Structures 27, 1262--1277 (2011).

[86] D. Poirel, M. Clément, H. Bouchard, and R. Vaivads. "Qualification of an Experimental Aeroelastic Apparatus at RMC." In "CASI 49th Annual Conference, Montréal, Canada," (2003).

[87] D. Poirel, Y. Harris, and A. Benaissa. "Self-sustained aeroelastic oscillations of a NACA0012 airfoil at low-to-moderate Reynolds numbers." Journal of Fluids and Structures 24(5), 700-719. ISSN 08899746 (2008).

[88] M. Gad-El-Hak. "Control of low-speed airfoil aerodynamics." AIAA Journal 28(9), 1537-1552. ISSN 00011452 (1990).

[89] T. J. Mueller. "Aerodynamic Measurements at Low Reynolds Numbers for Fixed Wing Micro-Air Vehicles." Mechanical Engineering (September), 13-17 (1999). 
[90] R. F. Huang and C. L. Lin. "Vortex shedding and shear-layer instability of wing at low-Reynolds numbers." AIAA Journal 33(8), 1398-1403. ISSN 00011452 (1995).

[91] J. Hall. Low Reynolds number aerodynamics for micro aerial vehicles. Ph.D. thesis, University of Colorado at Boulder (2007).

[92] I. Tani. "Low-Speed Flows Involving Bubble Separations." Progress in Aerospace Sciences 5, 70-104 (1964).

[93] R. Huang and H. Lee. "Effects of freestream turbulence on wing-surface flow and aerodynamic performance." Journal of aircraft 36, 965--972 (1999).

[94] R.-F. Huang, W. Shy, S. Lin, and F.-B. Hsiao. "Influence of surface flow on aerodynamic loads of a cantilever wing." AIAA Journal 34, 527-532 (1996).

[95] T. Yamashita, K. Yamashita, and R. Kamimura. "A Stepwise AIC Method for Variable Selection in Linear Regression." Communications in Statistics Theory and Methods 36(13), 2395-2403. ISSN 03610926 (2007).

[96] E. H. Dowell, H. C. Curtiss, R. H. Scanlan, F. Sisto, and R. M. Bennett. "A Modern Course in Aeroelasticity." Journal of Applied Mechanics 49(2), 465. ISSN 00218936 (1982).

[97] F. Herzog. "Brownian Motion and Poisson Process." (2010).

[98] P. E. Kloeden and E. Platen. Numerical Solution of Stochastic Differential Equations, volume 23 of Applications of Mathematics. Springer. ISBN 3540540628 (1992).

[99] S. Roweis. "Gaussian identities (http://www.cs.nyu.edu/ roweis/notes/gaussid.pdf)." 